\title{
Active living
}

Citation for published version (APA):

Van Kann, D. H. H. (2017). Active living: The role of the primary school environment on children's physical activity and sedentary behavior. [Doctoral Thesis, Maastricht University]. Datawyse / Universitaire Pers Maastricht. https://doi.org/10.26481/dis.20170309dvk

\section{Document status and date:}

Published: 01/01/2017

DOI:

10.26481/dis.20170309dvk

Document Version:

Publisher's PDF, also known as Version of record

\section{Document license:}

Unspecified

\section{Please check the document version of this publication:}

- A submitted manuscript is the version of the article upon submission and before peer-review. There can be important differences between the submitted version and the official published version of record.

People interested in the research are advised to contact the author for the final version of the publication, or visit the DOI to the publisher's website.

- The final author version and the galley proof are versions of the publication after peer review.

- The final published version features the final layout of the paper including the volume, issue and page numbers.

Link to publication

\footnotetext{
General rights rights.

- You may freely distribute the URL identifying the publication in the public portal. please follow below link for the End User Agreement:

www.umlib.nl/taverne-license

Take down policy

If you believe that this document breaches copyright please contact us at:

repository@maastrichtuniversity.nl

providing details and we will investigate your claim.
}

Copyright and moral rights for the publications made accessible in the public portal are retained by the authors and/or other copyright owners and it is a condition of accessing publications that users recognise and abide by the legal requirements associated with these

- Users may download and print one copy of any publication from the public portal for the purpose of private study or research.

- You may not further distribute the material or use it for any profit-making activity or commercial gain

If the publication is distributed under the terms of Article $25 \mathrm{fa}$ of the Dutch Copyright Act, indicated by the "Taverne" license above, 


\section{Active Living}

The role of the primary school environment on children's physical activity and sedentary behavior

Dave Van Kann

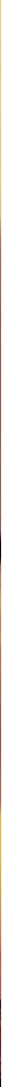


Credits

Production: Datawyse | Universitaire Pers Maastricht

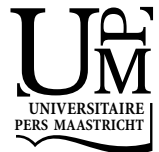

(C) Dave Van Kann, Maastricht, 2016

ISBN: 9789461596642

The research presented in this thesis was conducted at the School for Public Health and Primary Care (CAPHRI), Department of Health Promotion, Maastricht University. The work for this thesis was supported by the Academic Collaborative Centre for Public Health Limburg, a collaboration between 18 municipalities in Southern Limburg, the regional Public Health Service (GGD Zuid Limburg) and Maastricht University Medical Centre $(\mathrm{MUMC}+)$. The work was also supported by the Netherlands Organization for Health Research and Development (ZonMw), grant number 200130003. 


\section{Active Living}

\section{The role of the primary school environment on children's physical activity and sedentary behavior}

\section{Proefschrift}

ter verkrijging van de graad van doctor aan de Universiteit Maastricht, op gezag van de Rector Magnificus, Prof. dr. Rianne M. Letschert, volgens het besluit van het College van Decanen, in het openbaar te verdedigen op donderdag 9 maart 2017 om 16.00 uur.

door

Dave Helene Hub Van Kann

9 maart 1985, Kerkrade 


\section{Promotores}

Prof. dr. S.P.J. Kremers

Prof. dr. M.W.J. Jansen

Prof. dr. N.K. de Vries

\section{Copromotor}

Dr. S.I. de Vries (The Hague University of Applied Sciences)

\section{Beoordelingscommissie}

Prof. dr. Dike van der Mheen (Chair)

Dr. Patricia van Assema

Prof. dr. Martin Dijst (Utrecht University)

Prof. dr. Gerjo Kok

Dr. Esther van Sluijs (Cambridge University) 


\section{Contents}

$\begin{array}{lll}\text { Chapter } 1 \text { General introduction } & 7\end{array}$

Chapter 2 Active Living: development and quasi-experimental evaluation of a 19 school-centered physical activity intervention for primary school children

Chapter 3 The association between the physical environment of primary schools 35 and active school transport

Chapter 4 Parental Active Transportation Routines (PATRns) as a moderator of 49 the association between neighborhood characteristics and parental influences and active school transportation

Chapter 5 Playability of School-Environments and After-School Physical Activity 65 among 8-11 year-old Children: specificity of time and place

Chapter 6 Schoolyard characteristics, physical activity and sedentary behavior: 83 Combining GPS and accelerometry

Chapter 7 A multicomponent schoolyard intervention targeting children's recess physical activity and sedentary behavior: Effects after one year

Chapter 8 The effect of a school-centered multicomponent intervention on daily physical activity and sedentary behavior in primary school children: the Active Living study

Chapter 9 General discussion

References

Samenvatting

Summary

Valorization Addendum

Appendices

Dankwoord

Curriculum Vitae

Publication List 





Chapter 1

General introduction 



\section{SHAPING HEALTHY PHYSICAL ACTIVITY PATTERNS IN CHILDHOOD}

\section{Background}

The time spent by children in physical activity (PA) has decreased in recent decades, while the time spent in sedentary behaviors (SB) has increased substantially (Hallal et al., 2012; Hildebrandt, Bernaards, \& Stubbe, 2013; WHO, 2010). For children, the World Health Organization (WHO) recommends at least 60 minutes of moderate-to-vigorous physical activity (MVPA) a day (WHO, 2010). Only about ten percent of the children in Europe meet this recommendation (Verloigne et al., 2012), and the Dutch were found to be the most sedentary people in Europe (Bennie et al., 2013). The negative health outcomes caused by physical inactivity are well-studied (Kohl et al., 2012). Whereas physical inactivity has negative health consequences, PA is associated with multiple positive health outcomes (Janssen \& LeBlanc, 2010), with studies finding increased mental and physical well-being (Loprinzi, Cardinal, Loprinzi, \& Lee, 2012) and indications of better cognitive performances (Martin, Goryakin, \& Suhrcke, 2014; Singh, Uijtdewilligen, Twisk, van Mechelen, \& Chinapaw, 2012). Moreover, PA and SB in early life have been found to track into adulthood (Telama, 2009), i.e. being inactive as a child predicts inactivity in later life. These findings indicate the need to develop healthy physical activity patterns during childhood. Furthermore, increasing PA and decreasing SB in later stages of life might be more challenging than shaping healthy PA patterns during childhood (Verrotti, Penta, Zenzeri, Agostinelli, \& De Feo, 2014). The current decrease in PA and increase in SB among children underline the need for effectively intervening on childhood PA and SB. Developing effective interventions that encourage PA and decrease time spent in SB requires an improved understanding of these behaviors and their underlying determinants, which can be divided into personal determinants and environmental determinants. These personal and environmental determinants are both included in the so-called ecological approach.

\section{Ecological approach to encourage physical activity}

PA and SB are the outcomes of interplay between environmental and personal determinants. The ecological framework centers around the individual, surrounded by ecological levels that influence the individual's behavior (e.g. Bronfenbrenner, 1979; Sallis et al., 2006). Although environmental influences on an individual's behavior have been comprehensively demonstrated, clear pathways to explain and change behavior are typically lacking in these ecological frameworks. Dual-process models provide a clearer view of the pathways by which environmental, socio-cognitive and sociodemographic determinants relate to an individual's PA (Kremers, Visscher, Seidell, van Mechelen, \& Brug, 2005; Spence \& Lee, 2003). In the research reported on in this thesis, the view operationalized in the Environmental Research framework for weight 
Gain prevention (EnRG framework: Figure 1.1) has been adopted as the theoretical framework (Kremers et al., 2006). This framework identifies the environment, cognitive mediators, and moderators as relevant factors in the prediction of energy balancerelated behaviors, i.e. nutrition and physical activity, and provides indications for pathways by which the environment can influence physical activity. The primary focus of this thesis is the role of the school as a micro-level environmental setting and its role in influencing PA and SB among primary school children.

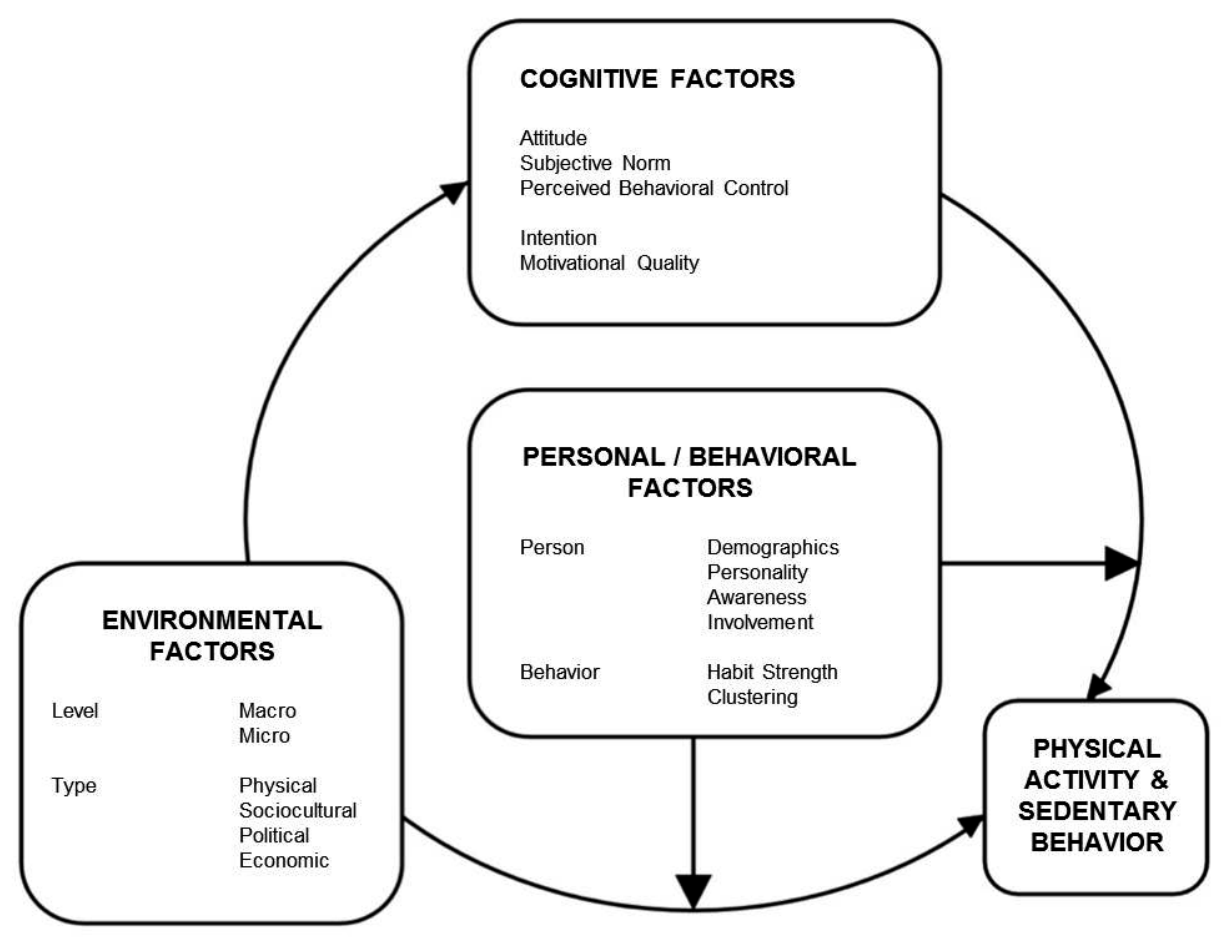

Figure 1.1: EnRG framework - version adapted for Active Living project (Kremers et al., 2006)

\section{Cognitive factors}

Well-studied socio-cognitive constructs are: attitude, subjective norm, perceived behavioral control, and intention, as operationalized in the Theory of Planned Behavior (TPB, Ajzen, 1991). The TPB constructs have mainly been studied in adult populations, and assume that PA and SB are both outcomes of a conscious process in which the motivation to be physically active or inactive, operationalized as intention, is the most proximal determinant of PA and SB. Intention refers to the motivation to engage in PA and is assumed to be influenced by the more distal PA determinants of attitude, subjective norm, and perceived behavioral control. The self-determination theory (SDT) emphasizes the importance of the nature of the motivation in predicting behavior, i.e. 
intrinsic and extrinsic motivation (Ryan \& Deci, 2000). Someone who is intrinsically motivated to perform PA is more likely to be physically active compared to extrinsically motivated people, i.e. if someone likes engaging in PA, he/she will be more likely to be active than someone who 'has to be' active for an external social or physical reward.

\section{Personal and behavioral factors}

Physical activity or inactivity can be influenced not only by socio-cognitive PA and SB determinants, but also by biological determinants and personality traits. Biological determinants of PA and SB include heritability, physical ability, weight status, movement skills, and physical fitness. In a recent review, Fisher, Smith, van Jaarsveld, Sawyer, and Wardle (2015) found that $21 \%$ of total daily PA could be explained by genetic influences. In addition, disabled children are at higher risk for physical inactivity and subsequent overweight (Rimmer \& Rowland, 2008). In addition, overweight children are at higher risk for physical inactivity compared to normal-weight children (Trost, Kerr, Ward, \& Pate, 2001), whereas children with well-developed fundamental movement skills have a lower risk of physical inactivity (Lubans, Morgan, Cliff, Barnett, \& Okely, 2010). PA can also be influenced by personality traits, such as impulsivity. Impulsive children may react differently to cognitive and environmental PA stimuli than nonimpulsive children, which affects their responses (Remmers, Sleddens, Kremers, \& Thijs, 2015).

Other factors that play an important role in predicting PA and SB are sociodemographic determinants related to PA and SB. The most thoroughly studied ones are: gender, age, ethnicity, and socio-economic status, including educational levels of the parents and (household) income. Male gender is associated with higher levels of PA (Verloigne et al., 2012), and age is strongly related to PA. While activity levels increase during childhood as a result of increased mobility and maturation, PA starts to decrease when children enter the pubertal phase and make the transition from primary school to secondary school (Morton et al., 2016).

In addition, children living in areas with a low-socioeconomic status (SES) are at increased risk for unhealthy behaviors, including physical inactivity (Baquet et al., 2014; Gordon-Larsen, Nelson, Page, \& Popkin, 2006; Wang \& Lim, 2012). Children living in low-income families have fewer opportunities to become physically active, for instance, because the physical environments in which these families live are often less PAsupportive than those in neighborhoods with a high SES (Gidlow \& Ellis, 2011). Finally, ethnicity has been identified as a determinant of PA and SB in Western countries, as belonging to an ethnic minority increases the likelihood of being physically inactive compared to native (Dutch), mostly white, children (Davidson, Simen-Kapeu, \& Veugelers, 2010). These differences can be partly explained by different cultural perceptions of health behaviors. In addition, ethnic minorities are generally less wealthy and therefore tend to live in low-SES neighborhoods, which negatively affects PA and SB (Gordon-Larsen et al., 2006). 
Within the family context, certain PA patterns appear to be present more or less as a routine (Kremers, Dijkman, de Meij, Jurg, \& Brug, 2008; Kremers \& Brug, 2008). These non-conscious, habitual behaviors can be the result of repeated exposure to environmental characteristics. For example, some inactive patterns, such as watching TV, can become habitual over time, simply as a result of repeated exposure to the TV (Ouellette \& Wood, 1998). Changing such habitual behaviors requires a different approach than changing behaviors that are the result of conscious processes.

\section{Environmental factors}

In addition to personal determinants, $\mathrm{PA}$ and $\mathrm{SB}$ are influenced by environmental factors. Environmental influences on PA (and SB) have gained more attention in recent decades. The environments in which children operate can be distinguished into four types (ANGELO framework, Swinburn, Egger, \& Raza, 1999): (1) the physical environment (the tangible built and non-built natural environment); (2) the sociocultural environment (social initiatives, normative social behaviors); (3) the economic environment (costs); (4) the political environment (policies and rules). Within these types of environment, a range of determinants can be identified that affect PA and SB. The research for the present thesis focused on influences in the physical and social environment in and around primary schools. Examples of determinants that belong to the physical environment are PA-supportive facilities such as the presence of sidewalks and other facilities for walking. The availability of destinations in the immediate neighborhood, such as parks and playgrounds, affect PA positively (Cohen et al., 2007; Floyd et al., 2011) as do well-maintained neighborhoods in terms of aesthetics and attractiveness (Sugiyama, Leslie, Giles-Corti, \& Owen, 2009). Moreover, a perception of greater safety (e.g. traffic safety) is associated with increased PA in school-aged children, both during transportation and during leisure time (Vanwolleghem et al., 2016). With regard to the socio-cultural environment, social behaviors, such as supportive practices by parents or teachers, tend to have a positive influence on children's PA levels (Davison, Li, Baskin, Cox, \& Affuso, 2011). The financial resources of families and legislation to provide sports lessons in school could be regarded as examples of environmental determinants in the economic and political categories.

\section{SCHOOL-BASED PHYSICAL ACTIVITY}

The primary school as a setting to promote healthy physical activity levels in children

All children spend a substantial amount of time at school during weekdays, so the potential impact of the school setting is large. Moreover, the school is a learning environment in which developing healthy PA patterns can be included in a curriculum. 
In practice, however, children spend most of their time in school in sedentary behavior during lessons. The only part of the school curricula where PA is embedded is that of physical education (PE) lessons. In most developed countries, primary schools follow recommendations that prescribe at least 120 minutes of PE per week. The recommendations for healthy MVPA in children certainly cannot be achieved by PE lessons only (Slingerland \& Borghouts, 2011). In the Netherlands, $75 \%$ of the primary schools schedule two PE lessons (45 minutes each) per week, and 20\% schedule only one lesson (Reijgersberg, Van der Werff, \& Lucassen, 2013). Additional PA opportunities in primary schools could be found in PA during recess (at schoolyards), after-school PA programs, and activities embedded in the 'regular' curriculum.

\section{Effectiveness of school-based PA interventions}

Many school-based PA interventions have been developed in the past. The effectiveness of these interventions for either active school transportation, (recess) PA at the schoolyard or PA after school has been reviewed by several researchers (e.g. Beets, Beighle, Erwin, \& Huberty, 2009; Broekhuizen, Scholten, \& de Vries, 2014; Dobbins, Husson, DeCorby, \& LaRocca, 2013; Ickes, Erwin, \& Beighle, 2013; Kriemler et al., 2011; Ridgers, Salmon, Parrish, Stanley, \& Okely, 2012a; Van Sluijs, Kriemler, \& McMinn, 2011). These reviews studied the effectiveness of specific intervention contents as standalone interventions (e.g. playground marking, providing equipment, policy/curriculum change) or as multicomponent interventions. The reviews largely focused on a supportive built environment, e.g. playground design at school, but their findings also suggested that socio-environmental interventions could be supportive in increasing PA and decreasing SB. At school, for instance, the availability of new fixed and portable playing equipment in schoolyards/playgrounds is a key factor in supporting PA (e.g. Ridgers, Fairclough, \& Stratton, 2010b; Stratton \& Mullan, 2005). Playground markings have been identified as a potential intervention to enhance PA in schoolyards (Blaes et al., 2013). As regards the social domain, several studies have reported positive results of teacher or coach involvement during schoolyard recess (e.g. Janssen, Twisk, Toussaint, van Mechelen, \& Verhagen, 2015). These teachers and/or coaches can either function as persons who encourage children to become or stay physically active or they can act as role models by performing PA themselves at schoolyards.

As regards active transportation to and from school, initiatives such as 'Safe Routes to School' and 'the Walking School Bus' have been developed to overcome issues of social (e.g. stranger danger) and traffic safety (e.g. dangerous intersections). Current reviews of these traffic related socio-environmental initiatives showed a positive effect of these interventions on the use of active school transportation (Smith et al., 2015; Stewart, Moudon, \& Claybrooke, 2014).

Reviews on the effectiveness of school-based PA interventions generally indicate that multicomponent interventions are more often implemented than single-element 
standalone interventions. The term 'multicomponent school-based intervention', however, is a generic term for interventions consisting of at least two intervention elements in the school setting, irrespective of the type of environment, i.e. physical or social environment. The tenets of the ecological perspective to allow interaction between multiple environmental levels and actors indicate that intervening on multiple types of environment would be beneficial (Gubbels, Van Kann, de Vries, Thijs, \& Kremers, 2014). This can be illustrated by the example of the schoolyard: creating a multicolored schoolyard, an intervention in the physical environment, and simultaneously introducing coaches at the schoolyard who encourage particular plays by children within each colored area at the schoolyard, an intervention in the socioenvironmental level, is hypothesized to have a larger effect on children's PA level than either of these intervention components alone. The interaction between types of environment and actors is illustrated by an approach such as the Comprehensive model for School PA Program (CSPAP), which focuses on multiple PA domains simultaneously (Hills, Dengel, \& Lubans, 2015).

The reported effectiveness of school-based PA interventions also depends greatly on the research designs used in studies, such as the duration of the follow-up period of interventions. Kriemler's umbrella review showed that $47-65 \%$ of the trials included in reviews showed positive results, but most trials that found interventions to be effective reported only effectiveness in the short term (varying between immediately after the intervention to up to 3 months). Only a few studies reported on the sustainability of the effectiveness of interventions (12 months and more). They reported them to be 'less' effective in the long term. The paucity of long-term effectiveness studies makes it more difficult to define whether school-based PA interventions have a sustainable impact on children's PA levels.

\section{Dutch primary school context}

In the Netherlands, children attend primary school from age 5 to age 12 years (grades 1-8) after which they transit to secondary school. A typical Dutch primary school day lasts from approximately 8.30 am to $3.00 \mathrm{pm}$ on Monday to Friday, with the exception of Wednesdays, when children finish school around $12.30 \mathrm{pm}$. Schools have a morning recess period of (mostly) 15 minutes and an afternoon (lunch) recess period that varies across schools (30-75 minutes), except for Wednesdays. As regards geographical characteristics, Dutch primary schools generally cater for a small residential area. The choice of a primary school is mainly driven by the proximity of the school (Bekkers, De Kool, \& Straten, 2012). This implies that primary school children mostly travel relatively small distances $(<1 \mathrm{~km})$, which could be considered feasible for active school transportation. 


\section{Active Living: development of the intervention}

The need to increase PA and decrease SB in children in an early stage of life, and the need for an effective school-based PA intervention led to the development of the Active Living project. Active Living took into account the considerations postulated by previous studies, such as focusing on multiple environmental influences and conducting long- (or longer-)term effectiveness studies. This project was launched at 10 primary schools in the Southern Limburg region of the Netherlands during the 2012-2013 schoolyear. Active Living used a context-dependent approach, i.e. it has the same scope to support PA and reduce SB in every school, but takes into account the local situation before developing and implementing any PA intervention. The Active Living project was designed according to the principles of systematic health promotion stated in the PRECEDE-PROCEED model (Green \& Kreuter, 1974). The project started with an analysis of health behavior, which indicated the need for childhood PA encouragement. Subsequently, we studied PA determinants and performed a needs assessment at each individual school. The specific interventions that fit in with this outline were designed for and targeted at the local needs defined by a local working group at participating schools. These working groups operated relatively autonomously to define their own set of interventions (bottom-up). Working groups were informed by researchers about scientific evidence for particular intervention elements (top-down). This approach resulted in a school-centered package of interventions aimed at increasing children's PA and reducing SB in three conceptual PA domains, i.e. active school transportation, PA at school, and PA during leisure time. The local tailored packages distinguish the Active Living project from PA interventions that implement interventions according to a 'onesize-fits-all' approach. The Active Living procedure enhances local support, hence leading to non-traditional analytic approaches to studying the effectiveness of these locally driven interventions. Finally, we conducted an effectiveness study as part of the PROCEED-pathway to evaluate the project.

\section{Content of Active Living and its contribution to the research field}

Interventions in the Active Living project focused on adjustments to the physical and social school environment in order to increase PA and reduce SB among primary school children. The environmental interventions had a shared scope, but the number, specific content, and magnitude of the interventions implemented could differ substantially between schools. A detailed overview of the design process of PA interventions in the Active Living project is provided in chapter 2 of this thesis.

Besides informing practitioners about applying a combined bottom-up, top-down approach to the development and effectiveness of school-based PA interventions, the Active Living project also offers scientific knowledge. Firstly, the Active Living study applied a quasi-experimental study design in which intervention schools were matched 
with control schools. In addition, multiple follow-up measurements are conducted. Applying this study design offered an opportunity to study the sustained effectiveness of tailored, multicomponent school-centered interventions implemented in terms of active school transportation, PA at school/schoolyards and PA during leisure time. Furthermore, it enabled analyses of the effectiveness of combined interventions implemented in different types of environment, i.e. the physical and social environment. The project also used several subjective and objective data collection instruments. Neighborhood audits and geographical information system (GIS) data were combined with objectively assessed PA data and GPS data. The innovative approach of combining accelerometry and GPS data further enhanced our understanding of the contextual, spatial environment in which PA and SB take place. With these assessment methods time- and location-specific analyses could be performed to examine the relationship between environment and behavior.

\section{RATIONALE OF THE PROJECT AND OUTLINE OF THIS THESIS}

\section{Rationale of this PhD project}

The rationale for the Active Living project was embedded in the scientifically grounded concept of the Academic Collaborative Center for Public Health, in which academia (including Maastricht University), local governments and the Public Health Service work together to study problems relevant to society. The development of Active Living was the result of a multidisciplinary collaboration between research, practice, and policy. The implementation of the project was mostly managed by practitioners (Southern Limburg Public Health Services) and policy makers (local municipalities), whereas the evaluation of the project was led by the research institutes involved (Maastricht University, the Netherlands Organisation for applied scientific research TNO, The Hague University of Applied Sciences). Involving practitioners and policy partners during the design process of the intervention was assumed to enhance the involvement of schools and local authorities in implementing multiple PA interventions. The study objectives for the research reported on in this thesis were therefore threefold: (1) developing, (2) implementing, and (3) evaluating an intervention to increase PA and to reduce SB among primary school children aged 8-12 years in Southern Limburg.

\section{Outline of the thesis}

The current thesis provides an overview of the development, implementation, and effect evaluation of the Active Living project, and reports on studies to improve our understanding of the relationship between environment and PA in primary school children. Chapter 2 describes the development and implementation of the Active Living 
interventions. Chapters 3 and 4 focus on PA during active school transportation (AST). Chapter 3 primarily describes the association between the physical school environment and AST, whereas chapter 4 mainly focuses on the moderating role of the social environment, i.e. parents, on the association between the physical environment and AST. Chapter 5 focuses on PA during leisure time. This study described the association between the playability of the neighborhood in which children live and children's afterschool PA levels. Furthermore, time- and location-specific analyses were introduced to study this association in greater depth. Chapters 6 and 7 present two studies conducted at schoolyards. Chapter 6 describes a study of the association between schoolyard characteristics and PA and SB in the schoolyard, using an innovative method of combining GPS and accelerometry. Chapter 7 discusses the effect of a multicomponent PA intervention implemented at schoolyards on children's recess physical activity levels. Chapter 8 brings together the three conceptual PA domains, i.e. AST, PA in school/schoolyard, and PA in leisure time, and presents the effects of the Active Living project on children's PA levels after 12 months. Finally, chapter 9 discusses the most important findings from the studies conducted and describes theoretical and methodological considerations, implications for future studies and recommendations for practice that can be inferred from this set of studies. 



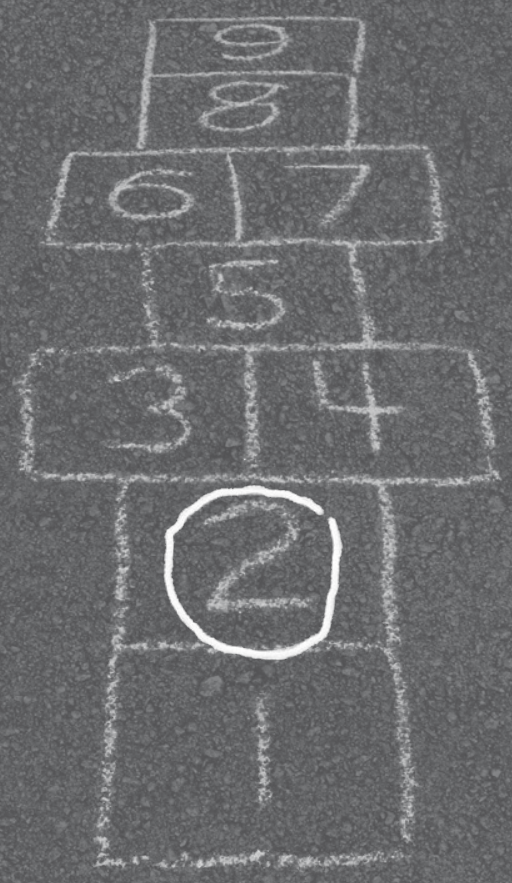

Chapter 2

Active Living:

development and quasi-experimental evaluation of a school-centered physical activity intervention for primary school children

This chapter has been published as: Van Kann, D. H. H., Jansen, M. W. J., de Vries, S. I., de Vries, N. K. \& Kremers, S. P. J. (2015). Development and quasi-experimental evaluation of a school-centered physical activity intervention for primary school children. BMC Public Health, 15:1315. 


\section{ABSTRACT}

Background: The worldwide increase in the rates of childhood overweight and physical inactivity requires successful prevention and intervention programs for children. The aim of the Active Living project is to increase physical activity and decrease sedentary behavior of Dutch primary school children by developing and implementing tailored, multicomponent interventions at and around schools.

Methods/Design: In this project, school-centered interventions have been developed at 10 schools in the south of the Netherlands, using a combined top-down and bottom-up approach in which a research unit and a practice unit continuously interact. The interventions consist of a combination of physical and social interventions tailored to local needs of intervention schools. The process and short- and long-term effectiveness of the interventions will be evaluated using a quasi-experimental study design in which 10 intervention schools are matched with 10 control schools. Baseline and follow-up measurements (after 12 and 24 months) have been conducted in grades 6 and 7 and included accelerometry, GPS, and questionnaires. Primary outcome of the Active Living study is the change in physical activity levels, i.e. sedentary behavior (SB), light physical activity (LPA), moderate-to-vigorous physical activity (MVPA), and counts-per-minute (CPM). Multilevel regression analyses will be used to assess the effectiveness of isolated and combined physical and social interventions on children's PA levels.

Discussion: The current intervention study is unique in its combined approach of physical and social environmental PA interventions both at school(yard)s as well as in the local neighborhood around the schools. The strength of the study lies in the quasiexperimental design including objective measurement techniques, i.e. accelerometry and GPS, combined with more subjective techniques, i.e. questionnaires, implementation logbooks, and neighborhood observations. 


\section{BACKGROUND}

All over the world, the prevalence of childhood obesity has increased substantially within one generation (Lobstein et al., 2015). As in most developed countries (Lobstein, Baur, \& Uauy, 2004), the proportion of overweight and obese children in the Netherlands has tripled between 1980 and 2009, to 16\% (Schönbeck et al., 2011). The prevalence of overweight is higher among children with a low socio-economic (SES) background (Ahrens et al., 2014; Brennan, Brownson, \& Orleans, 2014). Overweight in children is likely to track into adulthood (Parsons, Power, Logan, \& Summerbell, 1999; Singh, Mulder, Twisk, van Mechelen, \& Chinapaw, 2008), adding to the need for childhood overweight and obesity prevention. The increase in overweight is particularly caused by changed lifestyle behaviors, such as decreased physical activity (PA) and increased sedentary behavior (SB) (Kohl et al., 2012; Mitchell, Pate, Beets, \& Nader, 2013), which are nowadays considered two independent risk factors for health, rather than each other's counterparts (Pearson, Braithwaite, Biddle, van Sluijs, \& Atkin, 2014; Saunders, Chaput, \& Tremblay, 2014). Physical inactivity and sedentary behavior are associated with a wide variety of chronic diseases (Andersen et al., 2006), psychosocial problems (Biddle, Gorely, \& Stensel, 2004), and impaired cognitive functioning (Fedewa \& Ahn, 2011). Decreased PA and increased SB are likely to be a result of changed environmental factors, such as decreased road safety (Hume et al., 2009; Panter, Jones, \& van Sluijs, 2008) and increased availability of 'screen-based devices' (computers, televisions, tablets etc.) (Rush \& Simmons, 2014).

To date, there have been many interventions that aim to increase children's physical activity levels, especially in the school setting (Dobbins et al., 2013; Kriemler et al., 2011). Schools are suitable settings for health promotion activities in children in view of their substantial reach (Story, Nanney, \& Schwartz, 2009), which greatly affects the potential impact of an intervention. In addition, the educational system is a learning environment, in which developing a healthy lifestyle could be considered an important objective. Despite these potentials, however, schools are not able to solve the problem of physical inactivity by themselves (Koplan, Liverman, \& Kraak, 2005; Pate et al., 2006b). Nonetheless, most school-based PA interventions focus solely on the school setting (Broekhuizen et al., 2014; Escalante, García-Hermoso, Backx, \& Saavedra, 2014; Parrish, Okely, Stanley, \& Ridgers, 2013; Ridgers et al., 2012a), whereas it could be argued that school is only one level of influence (Sallis et al., 2006) and interactions with different ecological levels (e.g. home environment or local neighborhood) are more likely to change health behaviors (Gubbels et al., 2014).

The current project focuses on promoting children's PA levels (i.e. active school transportation, PA while attending school, and leisure time PA) in the school setting in a broad sense, including local neighborhoods and parts of the home environment. The Active Living project intends to create PA-friendly school environments by creating a supportive physical environment (e.g. safe routes to school and active schoolyards) 
accompanied by a supportive social environment (e.g. parental support to walk to school and facilitative teacher practices in schoolyards) (Figure 2.1). The interaction between interventions in both types of environments (Swinburn et al., 1999) is hypothesized to affect PA and SB favorably (Gubbels et al., 2014).

The aim of the Active Living project is to develop and implement tailored, multicomponent school-centered PA interventions and test their short- and long-term effectiveness regarding the PA levels of children aged 8-12 years living in deprived areas.

\section{METHOD}

\section{Setting and study design}

The current study uses a quasi-experimental design with 10 intervention schools and 10 matched control schools from the Southern-Limburg area in the Netherlands (Figure 2.2). It uses a combination of selective and universal prevention. Selective prevention is reflected by the focus on schools in deprived areas (Knol, 2012), with larger proportions of children from lower SES backgrounds. The project is, however, not only designed for low-SES children, but focuses on all children attending the schools in the deprived areas. 'Active Living' is funded by the Netherlands Organization for Health Research and Development (ZonMW), Project Number 200130003 (ISRCTN25497687). Ethical approval was obtained from the research ethics committee of the Maastricht University Medical Centre (reference number METC 12-4-077).

\section{Power calculation}

This project aims to increase children's physical activity levels. More specifically, its target is an increase in the proportion of children meeting the Dutch guideline for physical activity (60 minutes MVPA daily) from $22 \%$ to $42 \%$ after the project (De Vries \& Chorus, 2010; Ooijendijk, Wendel-Vos, \& de Vries, 2007). Interclass variations within the school (0.3) and variations among the schools (0.7) were taken into account in the power calculation. In total, we calculated that 704 children would be needed to obtain sufficient power. In order to achieve this we needed to include 16 schools (8 intervention and 8 control schools). Due to potential differences in class sizes and risk of drop-out during the study, the number of schools was increased by $25 \%$ to 20 schools (10 intervention and 10 control schools) and a total of 880 children.

\section{Recruitment of schools and participants}

The Active Living project targets $6^{\text {th }}$-to- $8^{\text {th }}$ grade children ( 8 to 12 years old) attending primary schools situated in deprived areas in the Southern-Limburg region. In 
November 2011, municipal development plans of all 19 Southern-Limburg municipalities were checked to see if they contained formal references to the themes of 'youth' and 'overweight prevention'. In total, 12 out of 19 municipalities had formulated targets either for youth or overweight prevention or both. Municipal health officers were visited and informed about the project and conditions for participation. This resulted in six municipalities (31.6\%) wanting to become involved. In these municipalities, four school corporations were identified, three of which agreed to recruit schools falling under their responsibility. To participate in the Active Living project, schools were checked for eligibility according to predefined inclusion criteria: (1) located in a deprived area; (2) at least 25 students enrolled in grades 6 and 7; (3) no plans to merge with another school or plans to relocate in the upcoming 3 years; and (4) willing to actively participate and to form an 'Active Living' working group at school. Within the municipalities and school corporations that consented, 37 primary schools were identified in deprived areas. Municipalities and corporations were asked to recommend schools that were most eligible to participate from their perspective. We visited 13 eligible schools and informed them about the project and conditions for participation; 10 schools (76.9\%) agreed to participate (Figure 2.3). Each of these schools was matched to a control school, taking into account the level of neighborhood deprivation and the level of urbanization (urban vs rural). Ideally, the control school was located in a non-participating municipality, to prevent potential contamination by municipal policy influences. After 10 matching control schools had been recruited, one control school unexpectedly had to relocate to a temporary school building and was thus replaced by an additional control school. In all, therefore, we included 11 control schools, 10 of which were matched to an intervention school. Control schools were only visited during measurements, and no project activities were planned during the intervention period, which comprised two academic years. Control schools were offered Public Health Services (PHS) support to implement effective elements of the project after the end of the effectiveness study.

Before data collection, all participating schools were visited to inform children about the study. Children were able to express their interest in participating in the effect study of the Active Living project. Those children who were interested were provided with written information for their parents. Written informed consent was requested from parents for wearing measurement equipment before and during the project. School principals consented to administer the child questionnaire in the classroom and to ask the parents to fill out the parental questionnaire. After permission was received from the parents to include a child in the study, the child could still refuse further participation in any part of the study without giving any reason. At baseline, parents of 815 children (61.6\%) consented for them to wear the measuring equipment. Figure 2.4 presents an overview of the participants of the Active Living project. 


\section{Data collection}

The baseline measurement (TO) of the Active Living Study was performed between September and December 2012. The first follow-up measurement (T1) was performed 6 months later, between March and June 2013, and a second follow-up (T2) after 18 months, between March and June 2014. To prevent potential seasonal effects, data in each pair of schools (the intervention school and the 'matched' control school) were collected on the exact same dates. Grades 6 and 7 of participating schools were visited by two researchers and a PHS employee at all measurement moments. During classroom visits lasting about 1 hour, children were instructed about the procedure of the measurement, were equipped with measurement devices (accelerometer and/or GPS device, see Measures), and individually filled out a questionnaire, which was immediately collected by the researchers. Afterwards, children received a parental questionnaire and were requested to ask their parents to fill it out and return it to school in a supplied envelope. A research assistant visited the schools one week afterwards to collect the measurement equipment and parental questionnaires.

Unexpectedly, the intervention implementation was delayed by at least 6 months as a result of reorganizations at our main executive partner (PHS). As a result, we revised the measurement design of our effectiveness study. The initial T0 measurement was redefined as a pilot and feasibility test (T-1). The measurement in the spring of 2013 was redefined as the baseline measurement (TO) while the original 18-month measurement became the first follow-up in the effectiveness trial (T1 = 12 months). As a result of this, we performed an additional follow-up measurement in the period of March till June 2015 (T2 = 24 months). This will allow us to assess the sustainability of potential effects of the Active Living project on the PA and SB behavior of the children who were in the $6^{\text {th }}$ grade at baseline. Children attending $7^{\text {th }}$ grade at the start of the project made the transition from primary to secondary school during the summer of 2014, and were therefore no longer eligible to participate in the additional 24-month measurement (T2).

The revised measurement design led to a loss of power in the study of long-term effects. On the other hand, it increased the comparability between the baseline (TO) and follow-up measurements ( $\mathrm{T} 1$ and $\mathrm{T} 2$ ), since all measurements were now performed in spring (while T-1 was performed in the fall). In addition, a potential novelty effect of wearing measurement devices was prevented by excluding T-1 from the effectiveness trial.

\section{Measures}

\section{Accelerometry and GPS}

PA was measured by waist-worn accelerometers (Actigraph $\mathrm{GT} 3 \mathrm{X}+, 30 \mathrm{~Hz}$ ). Children were instructed to wear a belt, with the attached accelerometer on their right hip, for at least 5 consecutive days, including a weekend. They were asked to wear the device 
all day long during waking hours and only remove the belt when performing (substantial) water-involving activities, such as swimming and showering.

At T-1 and at T0, a random selection of children were also asked to wear a GPS device (QStarz BT1000-XT), which was added to their accelerometer belt. We opted for this random approach because of the limited availability of GPS devices. Children additionally wearing a GPS device followed a similar protocol as described for accelerometers, but were asked to charge the device overnight using a supplied adapter. At T1 our GPS capacity was greatly increased, allowing us to ask all children to wear a GPS device in addition to their accelerometer. T2 was designed as an accelerometer-only measurement, to prevent high drop-out rates, as the GPS device was thought to raise the burden of participation for the children.

\section{Child questionnaire}

The child questionnaire included items that can be classified into six main themes (Table 2.1): demographics; PA and SB; neighborhood perceptions; rules and practices at home; PA preferences, and impulsivity (Rodenburg, Oenema, Pasma, Kremers, \& van de Mheen, 2013; Sleddens, Kremers, De Vries, \& Thijs, 2013; Timperio, Crawford, Telford, $\&$ Salmon, 2004) (Timperio et al., 2006). The questionnaire was pre-tested with children of the target age group to ensure the questions were clear and understandable. The questionnaire was designed to be completed individually by children within approximately 15 minutes. At T1, some process evaluation items were added to the questionnaire.

Table 2.1: Overview of concepts in the child questionnaire

\begin{tabular}{lll}
\hline Theme / Concept & N items & Example of items \\
\hline Demographics & 9 & Gender; Date of birth; Home address \\
Transportation options & 3 & How did you come to school this morning? (MC-9) \\
PA \& SB & 12 & $\begin{array}{l}\text { On how many days per week do you play outdoors? (Open) + } \\
\text { duration question }\end{array}$ \\
Neighborhood perceptions & 7 & There is heavy traffic in our local streets (Lik-4) \\
Rules and practices at home & 4 & $\begin{array}{l}\text { At home we have rules about using the computer or watching TV } \\
\text { (Lik-4) }\end{array}$ \\
$\begin{array}{lll}\text { PA preferences } & 28 & \text { Visualized comparisons, e.g. outdoor play vs. reading } \\
\text { Impulsivity } & 13 & \text { I often rush into new things (Lik-5) } \\
\text { Process } & 4 & \text { On a scale from 1-10, how much fun do you think Active Living } \\
\end{array}$ \\
\hline
\end{tabular}

MC-9 = Multiple Choice (9 options); Lik-X = X-points Likert Scale; SC = Scale

\section{Parental questionnaire}

The parental questionnaires included nine main topics (Table 2.2): demographics; school transportation choices; neighborhood perceptions; parental PA practices; child's outdoor play; parental active transportation routines (PATRns); rules; biking behavior, 
and self-reported height and weight of the child, mother, and father (Davison et al., 2011; Gattshall, Shoup, Marshall, Crane, \& Estabrooks, 2008). The questionnaire was designed to be completed in 10 minutes, which was believed to reduce the 'time barrier' to filling out the questionnaire.

Table 2.2: Overview of concepts in the parental questionnaire

\begin{tabular}{|c|c|c|}
\hline Theme / Concept & $\mathrm{N}$ items & Example of items \\
\hline Demographics & 11 & Relation to child; Level of education \\
\hline School transportation options & 3 & $\begin{array}{l}\text { Who accompanies your child during transportation to school? } \\
\text { (MC-6) }\end{array}$ \\
\hline Neighborhood perceptions & 8 & Social safety is a concern in our local neighborhood (Lik-4) \\
\hline \multicolumn{3}{|l|}{ Parental PA practices } \\
\hline Modeling & 6 & $\begin{array}{l}\text { How often do you use your own behavior to encourage your } \\
\text { child's PA? (Lik-5) }\end{array}$ \\
\hline Facilitation & 2 & $\begin{array}{l}\text { How often do you bring your child to a location where he/she is } \\
\text { able to do sports or be physically active? (Lik-5) }\end{array}$ \\
\hline Social Support & 8 & $\begin{array}{l}\text { How often do you stimulate your child to be physically active? } \\
\text { (Lik-5) }\end{array}$ \\
\hline Child's outdoor play & 10 & $\begin{array}{l}\text { If my child does not play outdoors, it is because.... there is no } \\
\text { playground in the local neighborhood (Lik-3) }\end{array}$ \\
\hline $\begin{array}{l}\text { Parental Active Transportation } \\
\text { Routines (PATRns) }\end{array}$ & 4 & $\begin{array}{l}\text { If I have to go somewhere nearby, I am always inclined to take the } \\
\text { bike or to go on foot (Lik-5) }\end{array}$ \\
\hline Rules & 4 & $\begin{array}{l}\text { How often do you restrict the time your child can use the } \\
\text { computer? (Lik-5) }\end{array}$ \\
\hline Biking behavior & 4 & How often do you cycle yourself? (Lik-5) \\
\hline Anthropometry & 6 & What is your height and weight? (Open) \\
\hline
\end{tabular}

MC-6 = Multiple Choice (6 options); Lik-X = X-points Likert Scale

\section{Environmental data}

In August 2012, prior to the start of the project at the schools, an environmental scan of all school environments was conducted using the SPACE checklist (De Vries, HopmanRock, Bakker, Hirasing, \& Van Mechelen, 2010). This is an adapted version of the Neighborhood Environment Walkability Scale (NEWS) (Saelens, Sallis, Black, \& Chen, 2003), specifically adapted to and validated for the Dutch context. A school environment was defined as an $800 \mathrm{~m}$ crow-fly buffer around every primary school. In 2012, Dutch primary school children lived at an average distance of $600 \mathrm{~m}$ from their nearest primary school (CBS, 2014), so the $800 \mathrm{~m}$ crow-fly buffer seemed appropriate to include both the school and home environment of the primary school children and their route to school. Two trained researchers conducted the environmental scan by walking through the neighborhood. Each scan took about 3-4 hours to complete. Environmental attributes that were audited were classified into: 'Schoolyard characteristics' (e.g. play equipment, green spaces, benches), 'Residential buildings' (e.g. numbers of apartment blocks), 'Sports facilities' (e.g. presence of sports fields), 'Playgrounds' (e.g. numbers of playgrounds, equipment at playgrounds, accessibility), 'Parks' (e.g. presence of park, 
play equipment in park, accessibility), 'Green spaces and water' (e.g. quantity, accessibility), 'Street networks' (e.g. presence of sidewalks, quality of sidewalks), 'Traffic safety in school vicinity' (e.g. presence of busy roads, heavy traffic), 'Street hygiene' (e.g. presence of litter, dog waste), 'Social safety' (presence of hang-outs, dark places), and 'Cyclability and walkability of neighborhood' (general impression of neighborhood). All study areas were revisited after T2 (12 months) to check for environmental changes. In addition, contextual information on the school environment (e.g. residential density) was 'enriched / supplemented' using the Geographic Information System (GIS) (ArcGIS, version 10.2, Top10NL). Furthermore, weather conditions, i.e. temperature, precipitation and hours of sunshine, were obtained for every hour of a measurement day from the Royal Netherlands Meteorological Institute (KNMI).

\section{Procedures of intervention development and implementation}

A combined top-down and bottom-up approach was used to develop and implement tailored, multicomponent school-centered PA interventions (Figure 2.1). The 'Active Living' project involved a project team that was composed of a research unit using the top-down approach and a practice unit using the bottom-up approach. Both units were based in the Academic Collaborative Center for Public Health, which facilitated continuous information exchange, leading to ongoing adjustments to the project while in progress. Subsequently, researchers conducted the environmental scans and trained three PHS employees in '(physical/social) environmental thinking', and provided evidence-based PA intervention opportunities. After the start of the project the research unit was involved in monitoring, catalyzing, and evaluating the process, and made adjustments when needed. The trained PHS employees composed a working group at each intervention school consisting of at least the PHS employee as chair, and representatives of the school, parents, and municipal authorities (ideally multidisciplinary, i.e. municipal officials from different disciplines/departments) and was complemented by other stakeholders when possible and necessary. The working groups started with a small budget of 2,000 euros each for a period of two school years. Informed by the environmental scan, this working group defined local needs. Based on the needs assessment, an intervention and sustainability plan was formulated, which was assessed by the project team before equipment or training facilities for the interventions were funded. If plans exceeded the budget, additional funding resources were applied for. After (financial) approval by the project team, intervention plans were implemented and monitored for their impact. Meanwhile the bottom-up loop was repeated for additional PA interventions. 


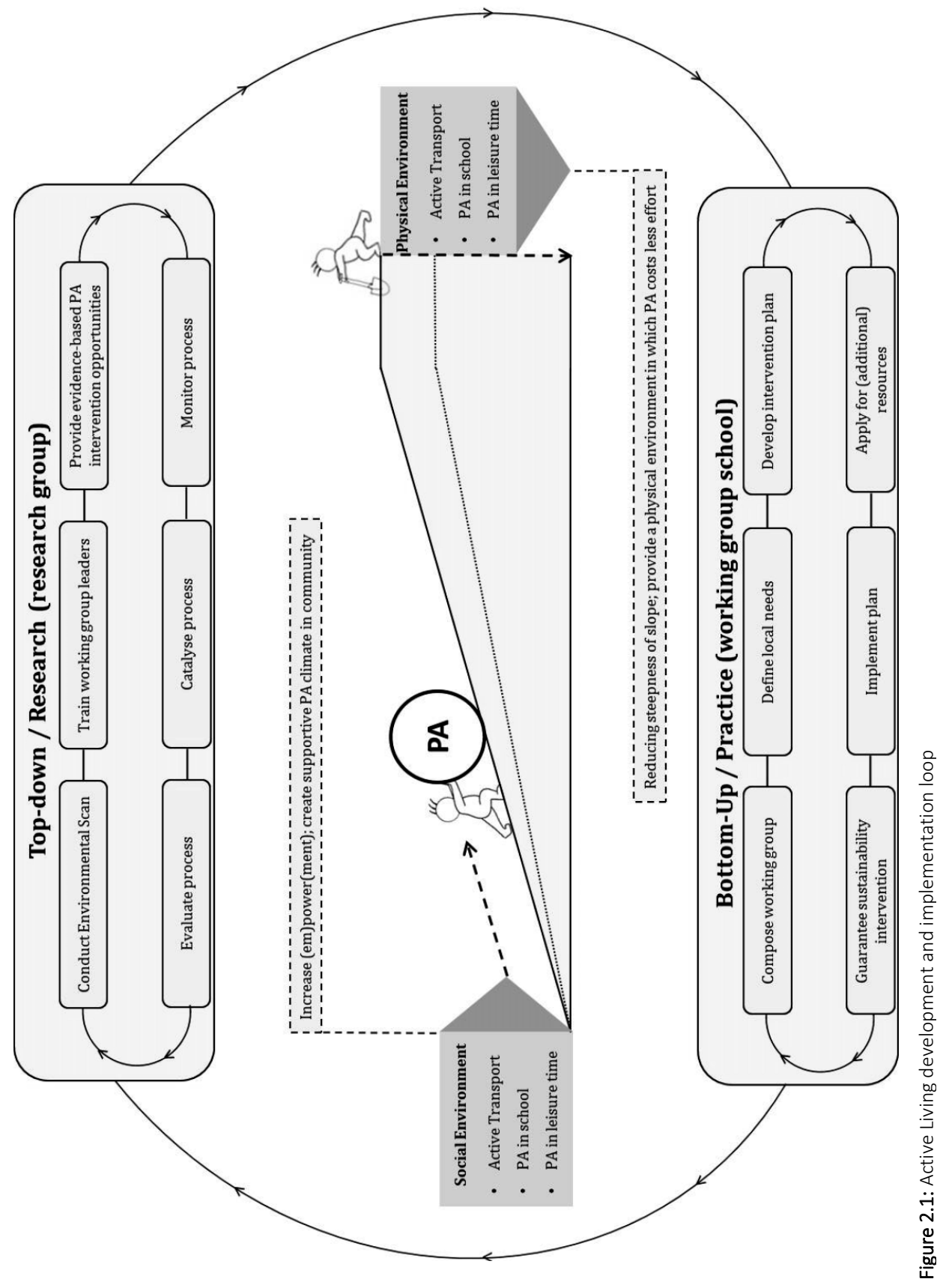




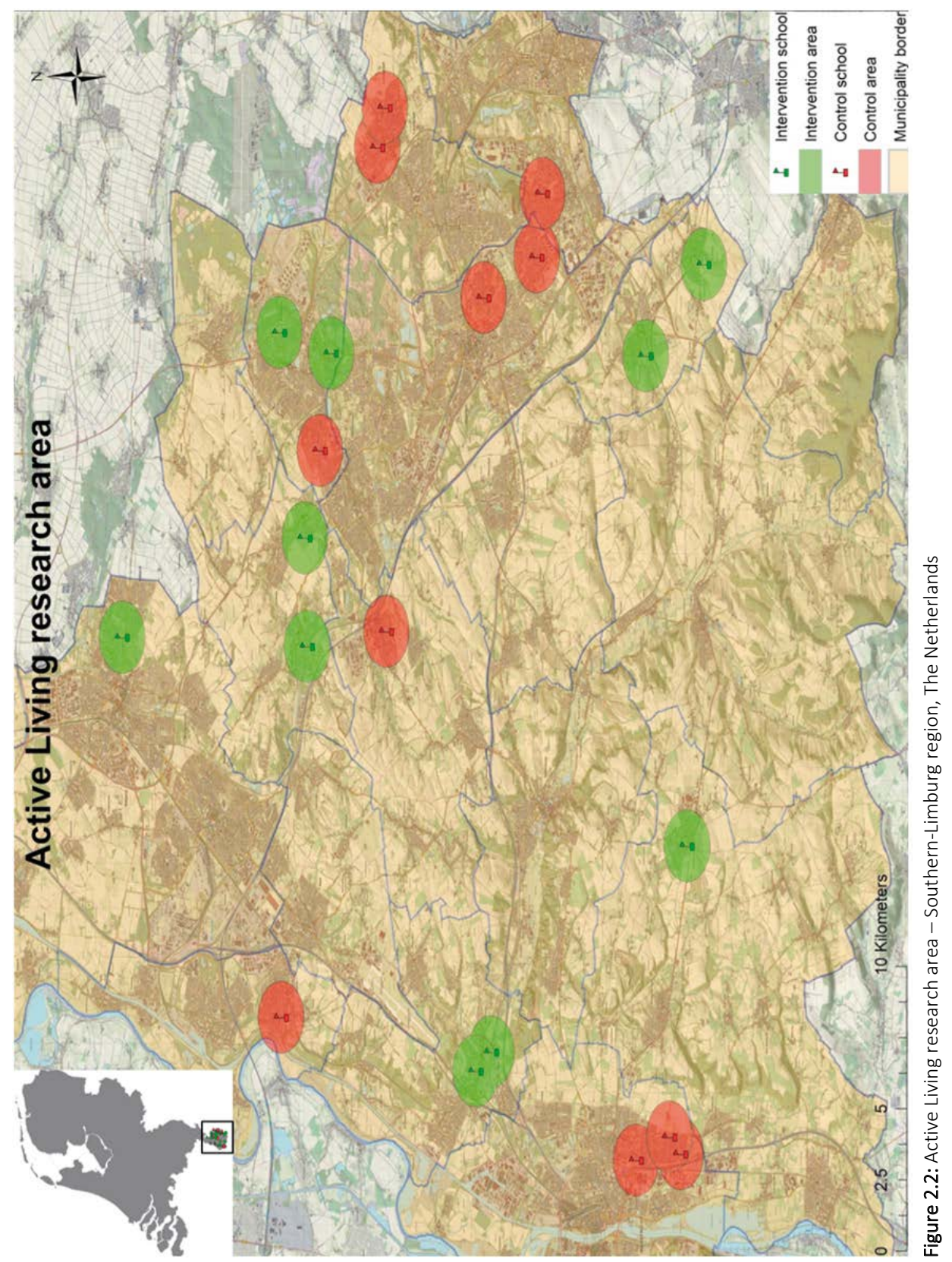




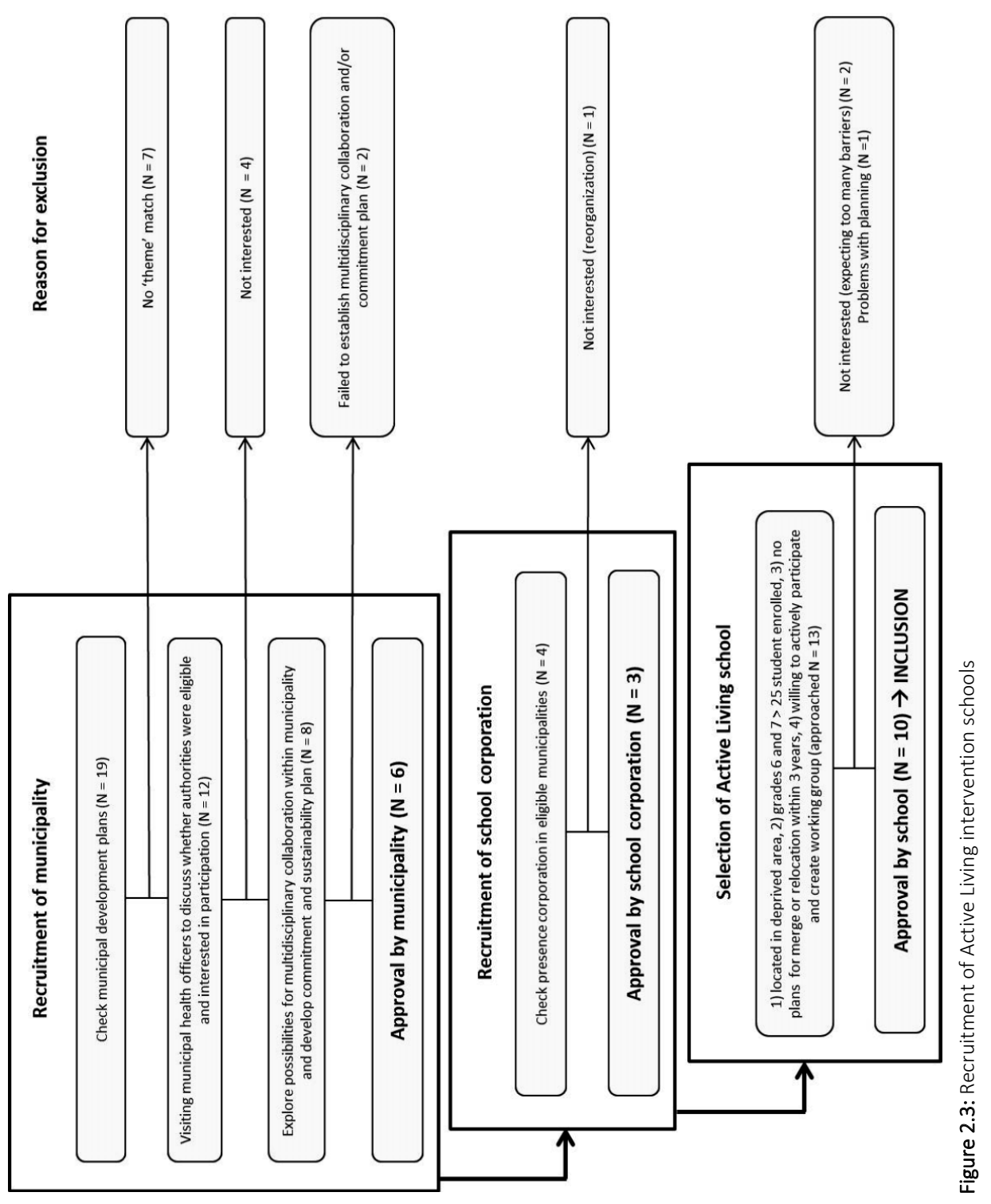




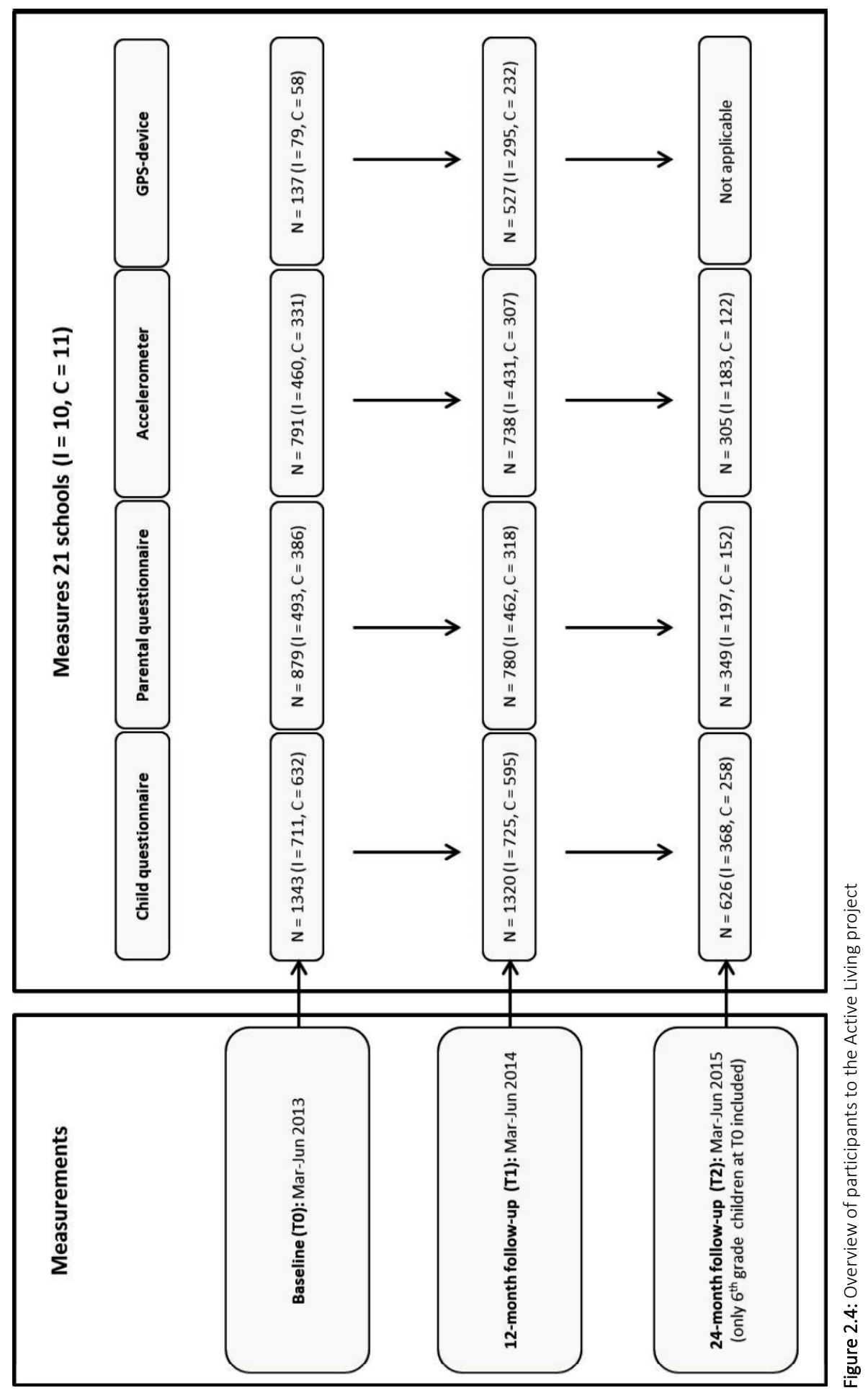




\section{Statistical analyses}

PA as measured with accelerometers will be described as activity counts-per-minute (CPM) and by activity levels, i.e. sedentary behavior (SB), light physical activity (LPA), and moderate-to-vigorous physical activity (MVPA). Activity levels will be classified using Evenson's cut-off values (Evenson, Catellier, Gill, Ondrak, \& McMurray, 2008). The primary outcome variables for the effectiveness study of Active Living will be the change in CPM $(\triangle \mathrm{CPM})$ and the change in time spent in SB, LPA, and MVPA ( $\triangle$ time spent in SB, LPA and MVPA, respectively) between T1 and the extended baseline measurement (TO). Differences between T2 and T1 and the extended baseline measurement (T0) will be analyzed to assess the sustainability of the Active Living intervention effects. Multivariate multilevel regression analyses will be used to adjust for the nested structure of data in schools. The regression analyses will also be adjusted for age, gender, ethnicity, and weather conditions. Intervention effects of multicomponent physical and social interventions will be studied, as well as their effectiveness under specific conditions, i.e. supportive school environments or home environments. The influence of moderators, such as child characteristics or parental practices, on the intervention effects on children's PA will be studied by including interaction terms of potential moderators in the models. In case of significant moderation, stratified analyses will be used to elucidate conditional factors.

Moreover, we intend to combine objectively (accelerometry and GPS) and subjectively (questionnaires) assessed measures to study the influence of environmental features (observed and perceived), children's characteristics (PA preferences and impulsivity), and parental practices on children's PA. Additional research questions have been formulated, such as 'Do PA preferences predict physical activity?' or 'What is the influence of parental PA practices on children's PA levels?'. The GPS data will allow us to zoom in on certain potentially important environments, such as schoolyards, playgrounds, or routes to school. Due to the limited number of GPS devices (at baseline), these location-specific data will not be part of the effectiveness study, but will be used for additional research questions, such as 'What neighborhood characteristics influence the use of active transportation?'.

\section{DISCUSSION}

The current paper has discussed the development and quasi-experimental evaluation of a school-centered PA intervention. In addition, we have introduced the plan of analysis to investigate the effectiveness of multilevel, multicomponent PA interventions on children's PA, which were developed and implemented using a combined top-down and bottom-up approach. We have also presented an overview of the recruitment procedures and 
measures, and elaborated on the research questions we will try to answer from our data, in addition to the main study objective.

The Active Living project involved developing and implementing a set of tailored physical and social environmental PA interventions. Every school had a different starting point for this study, and local needs varied greatly, both in focus (active transportation, PA at school, or PA in leisure time) and in content. The effectiveness of the Active Living project will be evaluated as a multilevel (individual, school, and/or neighborhood) multicomponent intervention. In this perspective, we focus on studying the effectiveness of changing the PA-friendliness of the whole school environment to enhance PA, rather than evaluating specific types of interventions. We will report on types of interventions implemented as part of Active Living to create a measure of the magnitude of the total set of physical and social interventions. We believe that studying the tailored development and implementation of a set of interventions in a combined top-down and bottom-up approach is one of the strengths of the Active Living project. Other strengths of the study are our measurements, including both objective assessment methods (accelerometry, GPS, and GIS in combination with environmental scans) and subjective methods (questionnaires among both children and their parents). The objective measurement techniques have already been tested in previous studies using similar target populations (Dessing et al., 2013; Klinker et al., 2014a; Klinker, Schipperijn, Kerr, Ersbøll, \& Troelsen, 2014b) and enable us to study children's actual physical activity behavior in a broader context, compared to previously used observation tools, such as SOPLAY and OSRAC-H (Mclver, Brown, Pfeiffer, Dowda, \& Pate, 2009; McKenzie, Marshall, Sallis, \& Conway, 2000).

The Active Living study has a quasi-experimental design. RCTs are generally considered to be the gold standard for testing the effectiveness of an intervention (Dobbins et al., 2013). For practical reasons, however, an RCT design seems less appropriate for field studies in which environmental changes are conducted in a wider, less controlled study area, as is the case in the Active Living project. Each of our study areas covers an environmental surface of $2 \mathrm{~km}^{2}$, certain parts of which will not be visited by all participants. Therefore, the exposure to interventions is more difficult to define than in studies focusing on one particular controlled area, such as a schoolyard. Besides, participants are well aware of whether they belong to an intervention school/area, since physical changes are directly visible to participants. Blinding is therefore practically impossible. Furthermore, adaptations in the public municipal domain have to be approved (and co-designed and co-funded) by local authorities and they may be key to the successful implementation of a participatory intervention strategy. Nonetheless, the quasi-experimental research design follows many of the RCT assumptions, and allows us to correct for potential confounding factors, such as sociocultural and political changes to the environment and weather conditions. Moreover, the quasi-experimental study design seems to be suitable for testing the effectiveness 
of interventions in daily practice, and therefore may facilitate the implementation and continuity of interventions.

\section{CONCLUSION}

The Active Living project contributes to the knowledge about the relationship between environmental interventions and physical activity. Active Living uses a participatory intervention development strategy, and multicomponent physical and social environmental PA interventions will be evaluated for their effects. The outcomes of the Active Living project will guide future projects to design or redesign schools, schoolyards, and their local environment. 


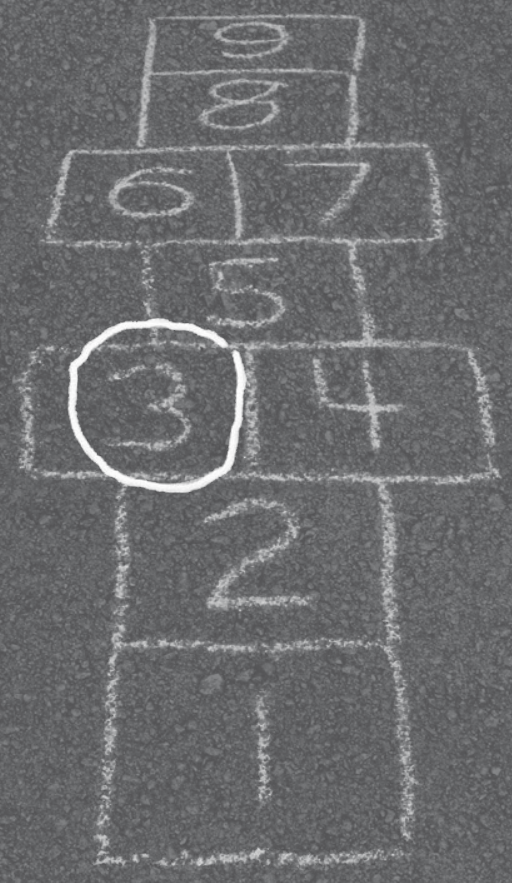

Chapter 3

The association between the physical environment of primary schools and active school transport

This chapter has been published as: Van Kann, D. H. H., Kremers, S. P. J., Gubbels, J. S., Bartelink, N. H. M., de Vries, S. I., de Vries, N. K. \& Jansen M. W. J. (2015). The association between the physical environment of primary schools and active school transport. Environment and Behavior, $47(4), 418-435$. 


\section{ABSTRACT}

This study examined the relationship between the physical environment characteristics of primary schools and active school transport among 3438 5-12 years-old primary school children in the Netherlands. The environmental characteristics were categorized into four theory-based clusters (function, safety, aesthetics, and destination). The correlations between the clusters and active school transport were examined and multilevel regression analyses were used to examine the association between the clusters and active school transport. No correlations were found between environmental clusters and active school transport for younger children (age 5-9), but for older children (age 9-12), strong positive correlations were found between aesthetics and active transport as were found for safety and active transport. School neighborhood aesthetics were related to active transport for older primary school children. Presence of parks, good maintenance of green spaces, and absence of litter in the school environment contributed most to the positive association between aesthetics and active school transport. 


\section{INTRODUCTION}

The prevalence of overweight and obesity is rapidly increasing among children, leading to physical problems such as type II diabetes and cardiovascular diseases in later life (Andersen et al., 2006) as well as psycho-social problems such as being teased and lower self-esteem (Biddle et al., 2004). Since overweight and physical inactivity patterns formed in childhood are major predictors of overweight in adulthood (Singh et al., 2008), special attention should be devoted to the prevention and treatment of childhood overweight. Nevertheless, time spent in physical inactivity and sedentary behavior is increasing worldwide (Kohl et al., 2012; Mitchell et al., 2013). In the Netherlands, only 30 to 40 percent of the children meet the recommended 60 daily minutes of moderate-to-vigorous physical activity (De Vries \& Chorus, 2010; WHO, 2010), and these proportions are even lower for children living in deprived neighborhoods (De Vries et al., 2005; Van Lenthe, Brug, \& Mackenbach, 2005).

Active school transport (e.g. walking and cycling) can make a major contribution to daily physical activity and to the development of active lifestyle patterns (Cooper, Jago, Southward, \& Page, 2012; Faulkner, Buliung, Flora, \& Fusco, 2009; Owen, Humpel, Leslie, Bauman, \& Sallis, 2004; Panter et al., 2008; Rennie, Johnson, \& Jebb, 2005; Van Sluijs et al., 2009). However, a decreasing proportion of children are using active transport, which is being replaced by motorized transportation to school (McDonald, 2007; Salmon, Timperio, Telford, Carver, \& Crawford, 2005; Van der Ploeg, Merom, Corpuz, \& Bauman, 2008). A range of possible determinants has contributed to the increased use of motorized school transport. Increased prosperity has led to an increased number of families that could afford owning a car and consequently make these people able to choose for motorized school transport. Furthermore, the perceived convenience of driving to school could have contributed to the decrease in active transportation (Trapp et al., 2012). Moreover, environmental characteristics (both physical and social) are likely to be important in determining this behavior, so identification of environmental correlates is essential to help reverse the downward trend (Panter et al., 2008; Tudor-Locke, Ainsworth, \& Popkin, 2001). Several studies (McMillan, 2007; Timperio et al., 2006) showed that the use of active school transport is inhibited by unsafe local neighborhoods, due to factors like traffic (e.g. busy roads, high traffic density) and social hazards (e.g. stranger danger and crime), while the distance to one's destination has also been found to be a major predictor of active transport use (McDonald, 2008; McMillan, 2007). Walking and cycling infrastructures, such as sidewalks and cycling paths, have also been reported to be environmental correlates of active school transport (Kerr et al., 2006).

Neighborhood characteristics can be structured and classified using socio-ecological models (Kremers et al., 2006; Pikora, Giles-Corti, Bull, Jamrozik, \& Donovan, 2003). Pikora's framework (Pikora et al., 2003) distinguishes four main environmental clusters related to walking and cycling in the local neighborhood. These neighborhood 
characteristics are clustered into function (e.g. physical attributes of the built environment), safety (e.g. social and traffic safety), aesthetics (e.g. access to interesting and pleasant physical environments), and destination (e.g. availability of community or commercial facilities in the neighborhood). Assembling the neighborhood characteristics into these theory-based clusters might be useful for understanding the local aspects associated with school transport.

The present cross-sectional study used Pikora's clustered approach to study the association between the school environment and the use of active transport to school among 5- to 12-year-old Dutch primary school children. This study focused on deprived areas, mostly inhabited by people in vulnerable positions and by children who were not fulfilling the recommended daily amount of physical activity (Uiters \& Verweij, 2010).

\section{METHODS}

\section{Description of research population}

This study focused on children from 19 primary schools situated in neighborhoods with a low socio-economic status (LSES) in five municipalities (i.e., Heerlen, Sittard-Geleen, Maastricht, Kerkrade, and Meerssen) in Southern Limburg, the Netherlands. School size ranged from 64 to 311 students. Children were between 5 and 12 years old, distributed over 8 grades (years). This study took place within the academic collaborative center for public health Limburg, in which the local municipalities, public health services and university work together intensively. The schools were invited by the local public health services to participate in this study, using mail and phone calls. The schools were asked to give permission for on-site visits to assess the number of children using active transport to come to school that day.

\section{Instruments}

Active School Transport (AST) use was determined by visiting each grade of the participating schools (grades 1 to 8), and asking the children 'How did you come to school this morning?' (by car / by bike / on foot / other). Final scores were reported as (1) active travel, i.e. by bike and on foot; or (0) motorized travel, i.e. by car and other. Children responded by raising their hands. The results were summarized for each school and grade into percentages of children using active versus motorized school transport. Children who were too young to cycle themselves, but were taken on their parent's bike, were counted as active travelers, whereas children who arrived on the back of their parents' scooter or motorcycle were categorized as motorized travelers. The transportation mode of a total of 3438 children was established. None of the children refused to answer. 
Characteristics of the physical environment were scored for a 400 meters radius around all participating schools, using the 'Environmental Scan for Active Transport to School' (ESATS) (Appendix 1; Table S3.1). The ESATS is based on the Spatial Planning and Children's Exercise (SPACE) checklist (De Vries et al., 2010), which is a Dutch version of the NEWS (Saelens et al., 2003). The NEWS has been adapted to correct for differences in the American and Dutch physical environment. Subsequently, the SPACE-checklist was adapted to record characteristics specifically related to active transport. In addition, a geographical adjustment (the presence of hills, (Timperio et al., 2006)) was made in the checklist, as this was specifically relevant for the current study area of Southern Limburg, resulting in ESATS. Presence of hills was included because they could be perceived as barrier for active transport. In our study sample, as is typical for the Dutch context, but even more typical for the study area of South Limburg, primary schools were covering a relatively small service area in homogeneous neighborhoods within the 400 meter buffer of each school (Figure 3.1). Although a pivotal focus has been paid to the physical environment of schools, generally a significant proportion of children's route to school as well as home neighborhood of children were included in the school neighborhood environmental audits.

Four clusters were created within the ESATS, based on the framework of physical environment factors by Pikora and colleagues: functional features (possible cluster score range: 0-73; example of an item: presence of cycling paths), safety features (0-42; e.g. presence of crossing aids), aesthetic features (0-29; e.g. presence of parks), and destination features (0-36; e.g. presence of bicycle sheds at school). Clusters were assembled by a theory-driven approach. The theory-based clusters were all correlated $<.7$ with each other (Table 3.2), i.e. each cluster explains less than half of the variance of another cluster.

Two observers assessed the school neighborhood environments. Each audit was performed by similar conditions in April 2011 during school hours. The audits took place within two weeks with comparable weather conditions; dry days with temperatures ranging from 11 to 21 degrees Celsius. Each scan took about 90 minutes to complete, and both assessors walked and cycled around the school areas to get a general impression of walkability and cycle-friendliness. Differences between the observers' scores were solved by discussion to reach consensus immediately after the observations took place. Reassessment of disputable characteristics was possible by immediate discussion of the observers' scores.

An SES score was calculated for each school, indicating the socio-economic status (SES) of the neighborhood in which the school is located. This score ranges from -4 (extremely deprived) to 4 (extremely well-off) and is based on mean household income, percentages of households with low income, percentages of residents without paid work, and percentages of households with a low educational level on average (Kohl et al., 2012) 


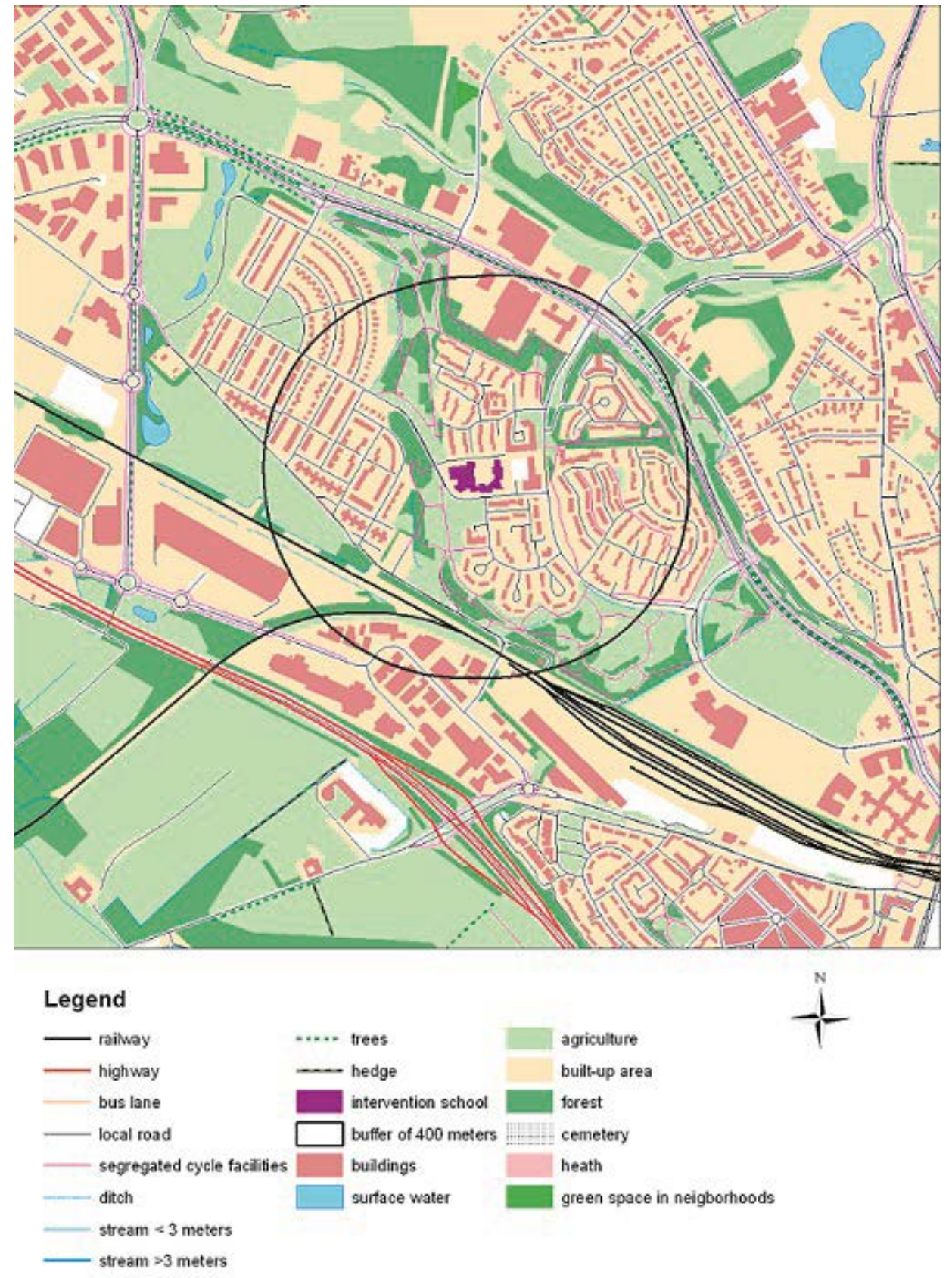

0 250 500 1.000

Figure 3.1: Example school neighborhood environment; 400 meters buffer 


\section{Statistical analyses}

Data were analyzed using SPSS, version 19.0 (SPSS Inc., Chicago). First, differences in use of active transport between lower grades ( 1 to 5 ) and higher grades (6 to 8) were assessed by an independent t-test, because differences in these subgroups were expected (Timperio et al., 2006). If a grade-related difference in active school transport was found, a dichotomous variable was constructed to discriminate between lower grades, in which school transport under parental supervision is likely, and higher grades. Associations between the ESATS clusters and active transport, for the entire sample as well as for both subgroups, were examined by calculating Pearson's correlation coefficients.

Multilevel regression analyses with two levels (school level and child level) were then conducted to examine the association between active transport and the ESATS clusters. We used backward deletion for the least significant cluster in each model, and the final model only contained statistically significant clusters $(p<.05)$. We also examined the intra-class correlation (ICC), which reflects the proportion of the total variation in active transport that is explained by the final model.

Subsequently, in-depth analyses of significant clusters in the final model were used to examine the associations between the individual characteristics included in the cluster and the use of active school transport. Similar to the analyses with ESATS clusters, two-level logistic multilevel regression analyses were performed, with all individual characteristics within the particular cluster as independent variables and active school transport as dependent variable, using the backward procedure.

\section{RESULTS}

The 19 participating primary schools had a socio-economic status score varying between -0.5 (slightly deprived neighborhood) and -2.7 (highly deprived neighborhood). The average number of children enrolled at each school was 181 (SD=69.3; Table 3.1).

Percentages of children traveling to school using active transport varied between $47.3 \%$ and $77.7 \%$ per school, with an average of $59.2 \%$ in the lower grades (1-5) and $73.8 \%$ in the higher grades $(6-8)(t(2438)=8.30, p<.01)$. Of the children who used active school transport ( $N=2170 ; 64.4 \%), 27.0 \%$ cycled to school $(N=585)$ and $73.0 \%$ walked to school $(\mathrm{N}=1585)$. 
Table 3.1: Demographics and active transport use (total and stratified) per school

\begin{tabular}{|c|c|c|c|c|c|}
\hline School & Munici-pality $^{1}$ & SES score $^{2}$ & $\%$ AST (N) & $\%$ AST grades 1-5 (N) & $\%$ AST grades $6-8(\mathrm{~N})$ \\
\hline 1 & 1 & -2.31 & $71.2(146)$ & $64.6(96)$ & $84.0(50)$ \\
\hline 2 & 2 & -2.69 & $73.4(64)$ & $65.8(38)$ & $84.6(26)$ \\
\hline 3 & 3 & -1.91 & $52.3(130)$ & $45.9(85)$ & $64.4(45)$ \\
\hline 4 & 1 & -2.31 & 76.5 (153) & $71.0(93)$ & $85.0(60)$ \\
\hline 5 & 1 & -2.31 & $67.8(311)$ & $66.1(186)$ & 70.4 (125) \\
\hline 6 & 1 & -2.08 & $57.9(297)$ & $54.8(188)$ & $63.3(109)$ \\
\hline 7 & 1 & -2.08 & $73.8(84)$ & $64.6(48)$ & $86.1(36)$ \\
\hline 8 & 1 & -1.97 & 48.7 (196) & $46.8(124)$ & $51.4(72)$ \\
\hline 9 & 1 & -1.54 & $74.1(166)$ & $70.5(95)$ & $78.9(71)$ \\
\hline 10 & 2 & -1.44 & $59.1(154)$ & $47.8(90)$ & $75.0(64)$ \\
\hline 11 & 2 & -1.44 & $69.9(236)$ & $65.2(164)$ & $80.6(72)$ \\
\hline 12 & 4 & -2.11 & $58.8(294)$ & $48.4(213)$ & $86.4(81)$ \\
\hline 13 & 5 & -0.46 & $66.9(133)$ & $61.7(81)$ & $75.0(52)$ \\
\hline 14 & 3 & -1.37 & 77.7 (103) & $76.3(76)$ & $81.5(27)$ \\
\hline 15 & 1 & -1.55 & 48.3 (207) & $42.1(133)$ & $59.5(74)$ \\
\hline 16 & 5 & -0.46 & 72.5 (167) & 69.4 (111) & $78.6(56)$ \\
\hline 17 & 4 & -2.11 & $47.3(182)$ & 44.1 (118) & $53.1(64)$ \\
\hline 18 & 3 & -1.83 & $59.6(188)$ & 56.1 (139) & $69.4(49)$ \\
\hline 19 & 3 & -1.13 & $67.8(227)$ & $63.0(138)$ & 75.3 (89) \\
\hline Total & & & $63.9(3438)$ & $59.2(2341)$ & 73.8 (1097) \\
\hline
\end{tabular}

${ }^{1}$ Municipalities; 1 = Heerlen, 2 = Sittard-Geleen, 3 = Maastricht, 4 = Kerkrade, 5 = Meerssen.

${ }^{2}$ SES score (ranging from -4 to +4 ) indicates the socio-economic status of the local neighborhood;

$\mathrm{N}=$ Number of children; AST = Active School Transport

Table 3.2 presents the correlations between all ESATS clusters, SES scores, and active transport at school level. Statistically significant positive correlations were found between safety and all other ESATS clusters, and between the aesthetic and destination clusters. Furthermore (although non-significant), SES was negatively correlated with local aesthetics and safety, but positively with function and destination.

All associations (except for SES) found between the school environment and active school transport were positive, i.e. physical environment scores were positively correlated with active transport. A positive association between aesthetics and active transportation, as well as for safety and active transportation, was found for the higher grades.

A moderating effect of lower and higher grades was found on the association between aesthetics and active transport (interaction term; $B=.083, p<.01$ ), so we performed two separate multilevel regression analyses for the subgroups. For children in the lower grades, all ESATS clusters were removed from the regression equation in the final model, indicating that none of the environmental clusters were associated with active transport. For children in the higher grades, aesthetics was significantly 
associated with active transport in the final model (Table 3.3). The odds ratio (OR) was 1.09 (95\% Cl=1.03-1.15) which means that the likelihood of active transport increased by $9 \%$ for each point of increase in the aesthetic cluster score. The proportion of the total variance (ICC) of active transport explained by aesthetics was $7 \%$.

Table 3.2: Pearson's correlations ( $r$ ) between ESATS clusters, SES score, and active school transport (AST) at school level $(\mathrm{N}=19)$

\begin{tabular}{|c|c|c|c|c|c|c|c|c|}
\hline & 1 & 2 & 3 & 4 & 5 & 6 & 7 & 8 \\
\hline 1 Function & & $.64^{* *}$ & .20 & .42 & .28 & .28 & .22 & .29 \\
\hline 2 Safety & & & $.58 * *$ & $.48^{*}$ & -.18 & .37 & .25 & $.47^{*}$ \\
\hline 3 Aesthetics & & & & $.68^{* *}$ & -.37 & .40 & .32 & $.54^{*}$ \\
\hline 4 Destination & & & & & .15 & .13 & .03 & .41 \\
\hline 5 SES score & & & & & & -.14 & -.18 & -.02 \\
\hline \multicolumn{9}{|l|}{6 AST grades 1-8 } \\
\hline \multicolumn{9}{|l|}{7 AST grades 1-5 } \\
\hline 8 AST grades $6-8$ & & & & & & & & \\
\hline
\end{tabular}

Note: SES score (range $-0.464--2.688) ;^{*}=p<.05 ; * *=p<.01$

Table 3.3: Stratified multilevel regression analyses for ESATS clusters and SES score for active school transport (AST)

\begin{tabular}{|c|c|c|c|c|c|c|c|c|}
\hline & \multicolumn{4}{|c|}{ Grades 1-5 $(\mathrm{N}=2341)$} & \multicolumn{4}{|c|}{ Grades 6-8 $(\mathrm{N}=1097)$} \\
\hline & \multicolumn{2}{|c|}{ Full model } & \multicolumn{2}{|c|}{ Final model $^{\mathrm{a}}$} & \multicolumn{2}{|c|}{ Full model } & \multicolumn{2}{|c|}{ Final model $^{\mathrm{a}}$} \\
\hline & $B$ & $p$ & $B$ & $p$ & $B$ & $p$ & $B$ & $p$ \\
\hline ESATS Function & .04 & .26 & & & -.01 & .75 & & \\
\hline ESATS Safety & -.03 & .60 & & & .07 & .39 & & \\
\hline ESATS Aesthetics & .08 & .14 & .03 & .21 & .10 & .13 & .09 & $<.01$ \\
\hline ESATS Destination & -.06 & .20 & & & -.02 & .67 & & \\
\hline SES score & .01 & .97 & & & -.03 & .30 & & \\
\hline
\end{tabular}

${ }^{a}$ Variables excluded using backward deletion for the least significant variable in each model

In-depth analyses within the aesthetic cluster showed that the presence of a park within the school neighborhood environment was the strongest correlate of active transport for children in the higher grades $(\mathrm{OR}=1.32,95 \% \mathrm{Cl}=1.09-1.54, \mathrm{p}=.02)$. In addition, the absence of litter on streets in the school environment was positively associated with active transport $(\mathrm{OR}=1.61,95 \% \mathrm{Cl}=1.17-2.06, \mathrm{p}=.03)$, as was good maintenance of local green spaces in this environment $(\mathrm{OR}=2.07,95 \% \mathrm{Cl}=1.43-2.72, \mathrm{p}=.04)$.

\section{DISCUSSION}

The current study examined the association between school neighborhood environment characteristics and the use of active transport by primary school children in the 
Netherlands. A total of $63.9 \%$ of the children in our sample used active transport, with higher proportions of older children doing so. School neighborhood characteristics were not found to be associated with active transport to school among young children, which is in line with studies conducted by Carver and colleagues (Carver, Timperio, \& Crawford, 2008a; Carver, Timperio, Hesketh, \& Crawford, 2010). They found environmental correlates of active transport only among older children. This might be explained by the dependence of this age group on the parents (Panter et al., 2008). Young children are mostly unable to travel to school by themselves, due to restrictions imposed by their parents or insufficient cycling or walking skills (Carver, Watson, Shaw, \& Hillman, 2013; Hume et al., 2009). A recent study by Carver showed that older children were licensed more frequently to use independent mobility to school and this license was positively associated with active transport (Carver et al., 2013). Parents are the main decision makers regarding the mode of transportation to school, and their environmental determinants of active transport use are likely to differ from the children's (Faulkner, Richichi, Buliung, Fusco, \& Moola, 2010; Panter et al., 2008; Timperio et al., 2004). For instance, time constraints may mean that parents are more inclined to use a car as a transportation method, regardless of environmental features around the school (Ahlport, Linnan, Vaughn, Evenson, \& Ward, 2008; Nelson, Foley, O'Gorman, Moyna, \& Woods, 2008). Trapp et al. (2012) found that the likelihood of active transport decreased when parents perceived driving their child to school to be more convenient, which implies that social determinants may be more important than physical environmental factors in predicting the use of active transport by younger children.

The strongest positive correlation between school neighborhood environment and active transport of older children was found for aesthetics. This implies that the attractiveness of the school neighborhood environment may influence the mode of transportation. An aesthetically more attractive environment has previously been found to relate to increased levels of physical activity (Veitch et al., 2011). In-depth analyses of the aesthetic cluster in our study showed that it was particularly the presence of a park in the school environment that was associated with active transport. Internationally, the presence of parks has mainly been associated with increased recreational physical activity (Cohen et al., 2007). In the Dutch school context, the presence of parks (or trails in parks) could be considered an important correlate of active transport. This finding is in line with the new urbanism philosophy of Calthorpe (1993) who endorses the creation of small neighborhood parks that provide diverse and pleasant scenery on route. Observational data does not suggest any destination effect of the presence of parks in school environmental neighborhood with regard to active transport, which endorses the urbanism philosophy that the pleasant scenery can contribute to this behavior. Good maintenance of local green spaces was also associated with active transport, in line with the review by Lee and Maheswaran (2011), who found that issues of maintenance affect the appeal of green spaces. The use or non-use of green spaces not only depends on its features but also on their condition. Another aesthetic 
characteristic which was positively associated with active transport was the absence of litter. Sugiyama and colleagues (2009) found that the attractiveness of the environment, including the absence of litter, was positively associated with walking by adults. The presence of litter has also been found to be negatively correlated with trail use (Reynolds et al., 2007). Both maintenance of local green spaces and absence of litter are contributing to pleasant scenery. Although it may be quite challenging to increase the number of parks within a densely populated residential area, the other two aesthetic environmental correlates, maintenance of local green spaces and absence of litter, may be less challenging to improve in school neighborhood environments, for instance by municipal regulations.

In addition to the importance of local aesthetic characteristics, we found a strong positive correlation between local safety and active transport use by older school children. Safety issues include mainly traffic safety, but also social safety such as vandalism. Traffic hazards, such as dangerous intersections and the lack of speed limits, have previously been reported by parents as a major barrier preventing them from giving their children permission to go to school on foot or by bike (Carver et al., 2010; Timperio et al., 2006; Weir, Etelson, \& Brand, 2006). Timperio and colleagues (2006) also found that increased traffic density is an important aspect of traffic safety and is a barrier to active transport.

In our multilevel regression analyses, the safety cluster was not significantly associated with active transport. However, in view of the strong correlation between safety and active transport, this does not imply that safety issues are unimportant, but its associative value might be less than in other international studies (e.g. in the USA and Australia), since several safety-related characteristics are present in almost all school neighborhood environments in the Netherlands, even in deprived areas (e.g. sidewalks, cycling paths, crossing guards, pedestrian crossings). The presence of such safety features in all school neighborhood environments may explain why we found relatively little variance within this cluster. Our findings can be interpreted on the basis of the hierarchical framework proposed by Alfonzo (2005). A certain threshold of safety characteristics in the school environment is an essential condition for the use of active transportation. Once these safety requirements are fulfilled, the aesthetic features of the school neighborhood environment might become more important correlates of active transport use. Other potentially important explanatory factors might be parental beliefs about active school transport or perceived health effects of active transportation.

Strong aspects of the present study include its sample size as well as the inclusion of children living in deprived areas. Compared to non-deprived areas, deprived areas are generally characterized by fewer features promoting physical activity and increased incivilities (Lee, Booth, Reese-Smith, Regan, \& Howard, 2005) as well as worse aesthetics, such as lower ratings of maintenance and decreased visual quality (Zhu \& Lee, 2008) that could decrease physical activity, including active transport. Further, a variety of school neighborhood environments were included, which resulted in 
adequate variance between school neighborhood environments. The clustered and multilevel analytic approach also provided added value, although the multilevel approach simultaneously limited the level-two variance to 19 school neighborhood environments.

A limitation is the assessment of active transport use, which was done by asking children to report their mode of transportation in class. Although De Wit and colleagues (2012) recently argued the validity of this method of collecting data, it may have led to social desirability bias. However, we have no indication that the children were pressured or biased to answer this question dishonestly. With regard to the assessment of active transport it should be noted that a distinction was made between active and motorized transport. This implies that children that were brought to school by bike by their parents, but were not bicycling themselves, were calculated as active travelers. This classification was underpinned by the strength of parental modeling behavior. It was expected that children whose parents use active transport to bring their child to school are likely to use active transport themselves when being physically able to travel independently (Murtagh, Rowe, Elliott, McMinn, \& Nelson, 2012). Due to the focus on age-related associations between the physical school neighborhood environment and active school transport, other demographic variables, such as gender, have not been included in the data-gathering process. Another shortcoming is that we were unable to calculate the distance between the children's home and school, since we were not able to track the addresses of the participating children. Distance can be a major barrier to active transport (Panter et al., 2008; Pont, Ziviani, Wadley, Bennett, \& Abbott, 2009) and the lack of this information could therefore have affected the associations we found between local school environment and active transport. However, this barrier is probably not as influential as it is in studies from other countries, as Dutch children generally live within a short distance (600 meters) of schools (CBS, 2014), a circumstance which may be considered favorable for active transport. Due to the proximity of primary schools in the Netherlands, most children typically go to the nearest primary school (Bekkers et al., 2012). Finally, a more detailed focus on all three environmental components of active school transport (school environment, route, and home environment) would be more informative compared to combining those components in school neighborhood environments. For this a more fine-grained instrument is needed than the ESATS-checklist, as well as the use of other network buffers and additional GPS- and GIS-data.

\section{CONCLUSION}

The association between local school neighborhood environment and the use of active school transport differed for younger and older Dutch primary school children. Associations between environmental features and active school transport were only 
found for older children, probably because younger children depend on their parents for transportation. Aesthetic and safety features in the school neighborhood environment were correlated with the use of active school transport among children aged 9 to 12 years. Factors especially associated with the use of active transportation by children included aesthetic characteristics, such as the presence of parks, good maintenance of green spaces, and absence of litter on the streets in the school environment, and these could therefore be regarded as potential aspects to intervene on. 



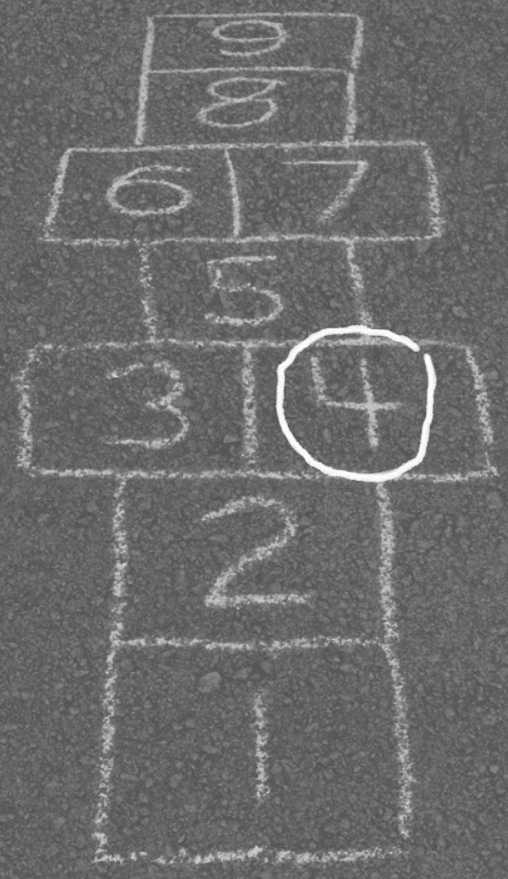

Chapter 4

Parental Active Transportation Routines (PATRns)

as a moderator of the association between neighborhood characteristics and parental influences and active school transportation

This chapter has been published as: Van Kann, D. H. H., Kremers, S. P. ., de Vries, S. I., de Vries, N. K. \& Jansen M. W. J. (2016). Parental Active Transportation Routines (PATRns) as a moderator of the association between neighborhood characteristics and parental influences and active school transportation. Environment and Behavior, 48(7), 946-965. 


\section{ABSTRACT}

Successful implementation of interventions to stimulate active school transportation (AST) requires better understanding of this behavior. This study explored the associations between Parental Active Transportation Routines (PATRns) and children's AST use, as well as the role of PATRns as a moderator of the association between the neighborhood characteristics and parental influences and AST. The study sample consisted of 722 8- to 12-year-old children and their parents living in the Netherlands. Multivariate linear regression analyses were performed, with the frequency of AST use as the dependent variable. Moderation by PATRns was tested by including interaction terms between PATRns and independent variables in the regression equation, and stratified analyses were conducted as a result of significant interactions. PATRns were a positive correlate of AST and were found to moderate the association between both parental facilitation of child physical activity and stranger danger and the use of active transportation, emphasizing the relevance of PATRns in increasing AST use. 


\section{BACKGROUND}

Active school transportation has been identified as an opportunity to increase daily physical activity (Cooper et al., 2012; Faulkner et al., 2009), as increasing numbers of children do not meet the recommended level of daily physical activity (De Vries \& Chorus, 2010; WHO, 2010). A substantial number of initiatives has been undertaken to increase the use of active transportation, but effects have been limited (Chillon, Evenson, Vaughn, \& Ward, 2011). Correlates and working mechanisms underlying the use of active school transportation need to become clearer if effective interventions are to be developed.

According to socio-ecological frameworks social environmental characteristics might interact with physical environmental characteristics in influencing behavior (Gubbels et al., 2014; Kremers, 2010), which indicates the need for focusing on the interactions rather than solely on separate main effects of physical and social characteristics on active transportation. Three major factors that could be involved in such interactions are physical environmental characteristics, social environmental characteristics, and routines or habits (Kremers, 2010).

Several attributes in the physical environment have been reported to be associates of active school transportation (Pont et al., 2009), such as distance (McDonald, 2008; Trapp et al., 2012), traffic safety related attributes (Wong, Faulkner, \& Buliung, 2011), and perceived neighborhood safety (Carver, Timperio, \& Crawford, 2008b; Kerr et al., 2006). However, even when most physical environmental preconditions for active transport are fulfilled, such as sidewalks and bicycle lanes, many children still do not use active transportation (Van Kann et al., 2015b), which is in line with the statement of Napier and co-workers (Napier, Brown, Werner, \& Gallimore, 2011) that creating a walkable environment is a necessary, but not sufficient condition to increase active school transportation.

In the first years of life, parents are the main decision makers with regard to transportation choices for their children (Carver et al., 2013; Hume et al., 2009; Panter et al., 2008; Pont, Ziviani, Wadley, \& Abbott, 2011) and could therefore probably be considered as the most important social environmental characteristics for children's mode of transportation to school. Parental modeling behavior of active transportation has recently been associated with children's active school transportation (Carlson et al., 2014). In Carlson's study however, modeling was conceptualized as engaging in any active travel by the parent. Several other important parental physical activity supporting behaviors or practices seem relevant (Edwardson \& Gorely, 2010; Hohepa, Scragg, Schofield, Kolt, \& Schaaf, 2007), such as supporting children by providing facilitation in terms of transport and paying fees for activities in which physical activity is involved, e.g. a sports club (Hoefer, McKenzie, Sallis, Marshall, \& Conway, 2001; Sallis et al., 1992) and providing emotional support (Davison, 2004). 
Active transportation can be regarded as a highly habitual behavior (Gardner, 2009; Murtagh et al., 2012). Habits have been conceived as learned sequences of acts that, as a result of frequent performance in similar situations, are triggered automatically by environmental cues (Ouellette \& Wood, 1998; Triandis, 1980). Based on the important position of parents with active school transportation choices, their own habitual engagement in active transportation could be a strong contextual factor for children's mode of transportation to school. Parental active transportation routines (PATRns) could thus interact with the influence of physical and social environment characteristics on children's active school transportation. PATRns reflect the extent to which parents use an active mode of transport instead of a motorized mode to destinations within a relatively short distance, such as posting a letter and going for a few groceries in the local neighborhood. The mode of transport for such short travels is considered as a routine behavior which is probably more automatically triggered rather than the result of an extensive deliberation process. PATRns refer to implicit messages that parents send out to their children, creating a family climate towards the use of active or motorized transportation. The working mechanism behind PATRns is thus more than just modeling parental behaviors, but also reflects implicit family norms, routines and habits. Including PATRns in current explanatory models for children's mobility to school could substantially contribute to explaining children's use of active school transportation and guide further development of effective interventions to increase active school transportation. Parental routines can be considered as a relatively stable contextual factor that varies widely across families.

The hypothesis that PATRns interact with the physical and social environment characteristics in determining active school transportation follows the tenets of habit theories, which assume that environmental features interact with habit strength in determining individual behavior (Triandis, 1977). Including a habitual component in explanatory models, i.e. PATRns, might provide a more comprehensive understanding of determinants underlying active transportation behavior (Gardner, Bruijn, \& Lally, 2011).

The purpose of the current study was to investigate the associations between the parental active transportation routines, PATRns, and children's active school transportation, as well as the association between physical and social neighborhood characteristics, parental physical activity support and active school transportation. Moreover, the focus of this study is to explore the role of PATRns as a moderator of the association between the physical and social neighborhood characteristics and children's active school transportation. It is hypothesized that for children who are raised in families with more exposure to active transportation (i.e. stronger PATRns), effects of supportive physical and social environments on active school transportation will be stronger and the effects of hindering environments weaker. 


\section{METHODS}

Data collection took place during the fall of 2012 in the Southern Limburg region of the Netherlands. All grades 6 and 7 of the 21 participating primary schools were visited (Dutch regular primary school system comprises grades 1 to 8). Each child completed a questionnaire focusing on their physical activity and the environmental attributes of physical activity in the classroom, while researchers were present to answer possible questions and to ensure comparability of contexts across classes. None of the children refused to fill in the questionnaire. All children received a questionnaire for their parents and these questionnaires were collected one week afterwards at school. The questionnaires focused on parental perceptions of the physical and social environment, parental physical activity support and parental active transportation routines (PATRns). The study was given ethical approval by the Medical Ethics Committee of the Maastricht University Medical Center (reference number METC 12-4-077).

\section{Measures}

The questionnaire data provided by the children were used to define the primary outcome variable in this study, i.e. the total number of days a week that children had used active school transportation in the previous week. Active school transportation was measured by the question 'On how many days in the past week did you come to school on foot or by bike?' This question resulted in a continuous variable ranging from 0 to 5 days a week. In addition, several demographic variables were assessed, including the children's age, grade, sex, home address, and country of birth. Parents were asked to report their household situation (i.e. single-parent household, two-parent household), level of education based on highest obtained degree (low education = lower vocational education or below; medium = between secondary vocational school and high school; high = higher professional education or university education), number of cars in the household, and country of birth (dichotomized into Western - nonWestern).

\section{Parental Active Transportation Routines (PATRns)}

PATRns were assessed in the parental questionnaire using four statements; 'If I have to go somewhere nearby, I am always inclined to take the bike or to go on foot', 'I often find myself using the car for a distance that could easily have been traveled by bike' (reverse coded so that high numbers describe less car use), 'Taking the bike to go somewhere is a habit for me', and 'Going somewhere on foot is a habit for me' (1-5 Likert scale; (1) totally disagree - (5) totally agree; Cronbach's $\alpha=.713$ ). Based on these items two scales were constructed; a PATRns total score (ranging 4-20) and a dichotomized scale indicating either strong or weak PATRns. Strong PATRns were 
defined as engaging in all dimensions of the PATRns scale (agree or strongly agree; 4 or 5); all other respondents were classified as having weak PATRns.

\section{Physical and social neighborhood characteristics}

Physical and social neighborhood characteristics were collected using a validated systematic observation instrument (SPACE checklist; (De Vries et al., 2010)). Observations were carried out by two trained observers. Inter-rater reliability (IRR) was substantial (Cohen's Kappa $=.73$ ( $p<.01)$ (Field, 2005; Landis \& Koch, 1977). A previous study in a similar Dutch context showed that the 'aesthetics' and 'safety' clusters were the strongest associates of children's active school transportation (Van Kann et al., 2015b), so neighborhood characteristics of these two clusters were observed and included in the analyses. In addition, the safety cluster was subdivided into social safety and traffic safety for the purpose of the current study. In total, 44 neighborhood characteristics were assessed in an 800 meter ( 0.5 miles) crow fly buffer around participating schools. For each cluster a sum score was calculated based on all items measured on aesthetics, social safety, or traffic safety. All sum scores were coded in a positive direction towards to the use of active transportation. An aesthetics score could range between 9 and 36 and was observed by items such as 'presence of neighborhood parks' and 'presence of litter'. Social safety could range between 4 and 22 and included items such as 'presence of street lightning' and 'presence of hang outs'. Finally, traffic safety was observed by items such as 'presence of traffic lights' and 'presence of speed humps' and could range between a score of 23 and 96. Distance to school was defined by calculating the distance in meters of the shortest walking route between the home address and the school address via Google Maps.

In addition to observed neighborhood characteristics, social neighborhood characteristics were defined by measuring parental perceived traffic load and parental perceived stranger danger, by presenting two statements. The statement for parental perceived traffic load was 'There is a lot of heavy traffic in the neighborhood I am living in' and the statement for parental perceived stranger danger was 'I am worried about strangers in the neighborhood I am living in' (1-5 Likert scale; (1) totally agree - (5) totally disagree) (Timperio et al., 2006).

\section{Parental physical activity support}

Parental physical activity support was measured by parental practices with regard to emotional support, facilitation, and modeling. A parental emotional support scale was constructed using four items; 'How often do you stimulate your child to be physically active?', 'How often do you stimulate your child to play outdoors?', 'How often do you offer your child choices for physical activity (PA)?', and 'How often do you praise your child for being physically active?' (1-5 Likert scale; (1) never - (5) very often; Cronbach's $\alpha=.674$, scale ranging from 4 to 20) (Davison et al., 2011). Parental facilitation was operationalized as providing logistic support to children for being physically active and 
was measured by two items; 'Do you facilitate your child in participating in sports or other activities that stimulate PA?' and 'Do you take your child to places where he/she can be physically active?' (1-5 Likert scale; (1) never - (5) very often; Cronbach's $\alpha=$ .682, scale ranging from 2 to 10) (Davison et al., 2011). Parental modeling practices were measured by six items; 'How often do you play sports or are you physically active together with your child?', 'How often do you use your own behavior as an example to encourage your child to engage in PA?', 'How often do you play sports or are you physically active yourself?', 'How often does your child see you being physically active?', 'How often does your child see you being physically active for recreation?', and 'How often does your child hear you talking about sports and PA?' (1-5 Likert scale; (1) never $-(5)$ very often; Cronbach's $\alpha=.747$, scale ranging from 6 to 30) (Davison et al., 2011).

\section{Data analysis}

All analyses were performed using SPSS version 20.0 (SPSS Inc., Chicago), with a p-value of .05 indicating statistical significance. Attrition analyses were conducted to study differences between children whose parents did return the parental questionnaire and children whose parents did not. No statistically significant differences were found between both groups.

All independent variables were correlated to each other to check for multicollinearity and potential suppressor effects. All correlations were $<.30$, and the variables were therefore considered to measure non-overlapping constructs, leading to inclusion of all constructs into the regression analyses.

A multilevel structure of the data based on school level was tested by calculating the ICC of a random intercept model, revealing an ICC of 0.0376 , indicating a non-nested data structure. Therefore, analyses were conducted without correction for multilevel structure.

Multivariate linear regression analyses were conducted using the enter method. The number of days of using active transportation was included as the dependent variable, and demographics, physical and social neighborhood characteristics, parental physical activity support, and PATRns were entered as independent variables. PATRns were included as a continuous variable. Moreover, PATRns were included in the analyses as a potential moderating factor of the association between the other independent variables and active school transportation by computing interaction terms of all constructs with the continuous PATRns variable. By backward deletion (least significant interaction term was deleted first) interaction terms were eliminated. In case of significant interaction terms in the final model, stratification by PATRns (strong vs weak) took place. Multivariate linear regression analyses as described above were then repeated for both strata. 


\section{RESULTS}

\section{Description of research population}

In total, $13276^{\text {th }}$ and $7^{\text {th }}$ grade students completed a questionnaire distributed among 21 primary schools in the South Limburg region in the Netherlands and 817 of these children's parents completed the parental questionnaire (62\%). Matching children to their parents resulted in 735 valid child-parent matches of which 13 matches were excluded based on invalid answers on key variables, resulting in 722 child-parent matches in our study (54\%). The children's mean age was 9.68 (SD=0.72) years, ranging between 8 and 12 years. The gender distribution was almost equal and 95\% of the children had a Western background. Fifty-nine percent $(\mathrm{N}=420)$ of the parents had a medium (i.e. vocational to high school degree) level of education, and $81 \%$ of the children lived within a suitable distance from school to use active transportation (Table 4.1), which was defined as 1.2 kilometers or 0.75 miles (about 15 minutes of walk based on a walking speed of $5 \mathrm{~km} / \mathrm{h}$ per hour).

The strong PATRns group consisted of 122 child-parent matches while the group with weak PATRns consisted of 600 child-parent matches. We found two significant differences in independent variables between these groups (Table 4.2). Children included in the strong PATRns group lived closer to schools compared with matches in the weak PATRns group. Furthermore, parents in the strong PATRns group indicated modeling behavior for PA more often compared with parents in the weak PATRns group. The distribution of children did not differ across 21 schools for the strong and weak PATRns groups.

\section{Associates of active school transportation}

Distance to school was negatively associated with active transportation, i.e. the longer the commute distance the less often children actively traveled to school. PATRns were positively associated with active school transportation as was attending a higher grade. There was also a small positive association between traffic safety and active school transportation (Table 4.3). In total, this model explained over $26 \%$ of the variance. Distance to school explained $17 \%$ of the variance in the model, while PATRns explained over $3 \%$, school grade almost $2 \%$, and traffic safety about $1 \%$. No parental physical activity support scales were significantly associated with active school transportation (Table 4.3). 
Table 4.1: Description of the study population

\begin{tabular}{|c|c|}
\hline \multicolumn{2}{|l|}{ Total $(\mathrm{N}=722)$} \\
\hline Variable & $N(\%)^{*}$ \\
\hline \multicolumn{2}{|l|}{ Gender } \\
\hline Boys & $340(47.6 \%)$ \\
\hline Girls & $375(52.4 \%)$ \\
\hline \multicolumn{2}{|l|}{ Grade } \\
\hline 6 & $384(53.4 \%)$ \\
\hline 7 & $335(46.6 \%)$ \\
\hline \multicolumn{2}{|l|}{ Household situation } \\
\hline One-parent household & $94(13.1 \%)$ \\
\hline Two-parent household & $626(86.9 \%)$ \\
\hline \multicolumn{2}{|l|}{ Educational level of parents } \\
\hline Low & $57(8.0 \%)$ \\
\hline Medium & $420(58.6 \%)$ \\
\hline High & $239(33.4 \%)$ \\
\hline \multicolumn{2}{|l|}{ Ethnicity of parents } \\
\hline Western & $688(95.3 \%)$ \\
\hline Non-Western & $34(4.7 \%)$ \\
\hline \multicolumn{2}{|l|}{ Distance to school } \\
\hline 0-400 meters & $134(19.0 \%)$ \\
\hline 400-800 meters & $255(36.2 \%)$ \\
\hline 800-1200 meters & $178(25.3 \%)$ \\
\hline $1200-1600$ meters & $47(6.7 \%)$ \\
\hline \multirow[t]{2}{*}{$>1600$ meters } & $90(12.8 \%)$ \\
\hline & Means (SD) \\
\hline Parental Active Transportation Routines (PATRns) & $11.03(4.29)$ \\
\hline \multicolumn{2}{|l|}{ Physical and social neighborhood characteristics } \\
\hline Aesthetics (9-36) & $21.80(2.52)$ \\
\hline Social safety (4-22) & $16.49(2.69)$ \\
\hline Traffic Safety (21-96) & $62.10(4.65)$ \\
\hline Perceived traffic load (0-2) & $0.80(0.92)$ \\
\hline Perceived stranger danger $(0-2)$ & $1.08(0.88)$ \\
\hline \multicolumn{2}{|l|}{ Parental physical activity support } \\
\hline Facilitation (2-10) & $8.37(1.35)$ \\
\hline Emotional support (4-20) & $15.53(2.42)$ \\
\hline Modeling (6-30) & $19.39(4.27)$ \\
\hline
\end{tabular}

* $\mathrm{N}$ is based on valid cases; missing continuous values replaced by means; missing categorical values replaced by median 
Table 4.2: Description of strong and weak Parental Active Transportation Routines (PATRns) strata and differences between strata

\begin{tabular}{|c|c|c|c|}
\hline Variable & $N(\%) *$ & $\mathrm{~N}(\%)^{*}$ & t-value (df) \\
\hline Gender & & & N.S. \\
\hline Boys & $59(48.8 \%)$ & $281(47.3 \%)$ & \\
\hline Girls & $62(51.2 \%)$ & $313(52.7 \%)$ & \\
\hline Grade & & & N.S. \\
\hline 6 & $63(52.1 \%)$ & $321(53.7 \%)$ & \\
\hline 7 & $58(47.9 \%)$ & $277(46.3 \%)$ & \\
\hline Household situation & & & N.S. \\
\hline One-parent household & $13(10.7 \%)$ & $81(13.5 \%)$ & \\
\hline Two-parent household & $108(89.3 \%)$ & $518(86.5 \%)$ & \\
\hline Educational level of parents & & & N.S. \\
\hline Low & $8(6.6 \%)$ & $49(8.2 \%)$ & \\
\hline Medium & $69(57.0 \%)$ & 351 (59.0\%) & \\
\hline High & $44(36.4 \%)$ & $195(32.7 \%)$ & \\
\hline Ethnicity of parents & & & N.S. \\
\hline Western & $115(94.3 \%)$ & $573(95.5 \%)$ & \\
\hline Non-Western & $7(5.7 \%)$ & $27(4.5 \%)$ & \\
\hline Distance to school & & & $4.354+(d f=222)$ \\
\hline $0-400$ meters & $25(20.8 \%)$ & $109(18.7 \%)$ & \\
\hline $400-800$ meters & $56(46.7 \%)$ & 199 (34.1\%) & \\
\hline $800-1200$ meters & $32(26.7 \%)$ & $146(25.0 \%)$ & \\
\hline $1200-1600$ meters & $2(1.7 \%)$ & $45(7.7 \%)$ & \\
\hline \multirow[t]{2}{*}{$>1600$ meters } & $5(4.2 \%)$ & $85(14.6 \%)$ & \\
\hline & Means (SD) & Means (SD) & \\
\hline \multicolumn{4}{|c|}{ Physical and social neighborhood characteristics } \\
\hline Aesthetics (9-36) & $22.16(2.38)$ & $21.73(2.55)$ & N.S. \\
\hline Social safety (4-22) & $16.23(2.95)$ & $16.54(2.63)$ & N.S. \\
\hline Traffic Safety (21-96) & $62.26(4.99)$ & $62.07(4.58)$ & N.S. \\
\hline Perceived traffic load (0-2) & $0.81(0.94)$ & $0.80(0.92)$ & N.S. \\
\hline Perceived stranger danger $(0-2)$ & $1.16(0.87)$ & $1.07(0.87)$ & N.S. \\
\hline \multicolumn{4}{|l|}{ Parental physical activity support } \\
\hline Facilitation (2-10) & $8.21(1.36)$ & $8.40(1.35)$ & N.S. \\
\hline Emotional support (4-20) & $15.62(2.31)$ & $15.51(2.45)$ & N.S. \\
\hline Modeling (6-30) & $21.55(3.41)$ & $18.96(4.29)$ & $7.294+(d f=207)$ \\
\hline
\end{tabular}

$* N$ is based on valid cases; $\dagger^{+}=$significant difference strong and weak PATRns strata $(p<.05)$

\section{PATRns as a moderator}

PATRns were found to significantly moderate the association between parental physical activity facilitation $(\beta=.266 ; p<.01)$ and perceived stranger danger $(\beta=-.593 ; p<.01)$ and the use of active school transportation. Stratification on the PATRns (strong vs weak) revealed that facilitation was significantly associated with active school transportation in both strata, whereas this effect was not found when the total sample was analyzed (i.e. without stratification by PATRns). For parents with strong PATRns, parental physical activity facilitation was significantly and positively associated with active transportation. By contrast, if parents had weak PATRns, the association between 
parental physical activity facilitation and active transportation was significantly negative (Table 4.3). The association between perceived stranger danger and active school transportation was also moderated by PATRns. In the weak PATRns group, a marginally significant positive effect was found, i.e. the less perceived stranger danger in the school environment, the more the children were inclined to use active transportation. In the strong PATRns group, however, perceived stranger danger was not found to be associated with active school transportation. 


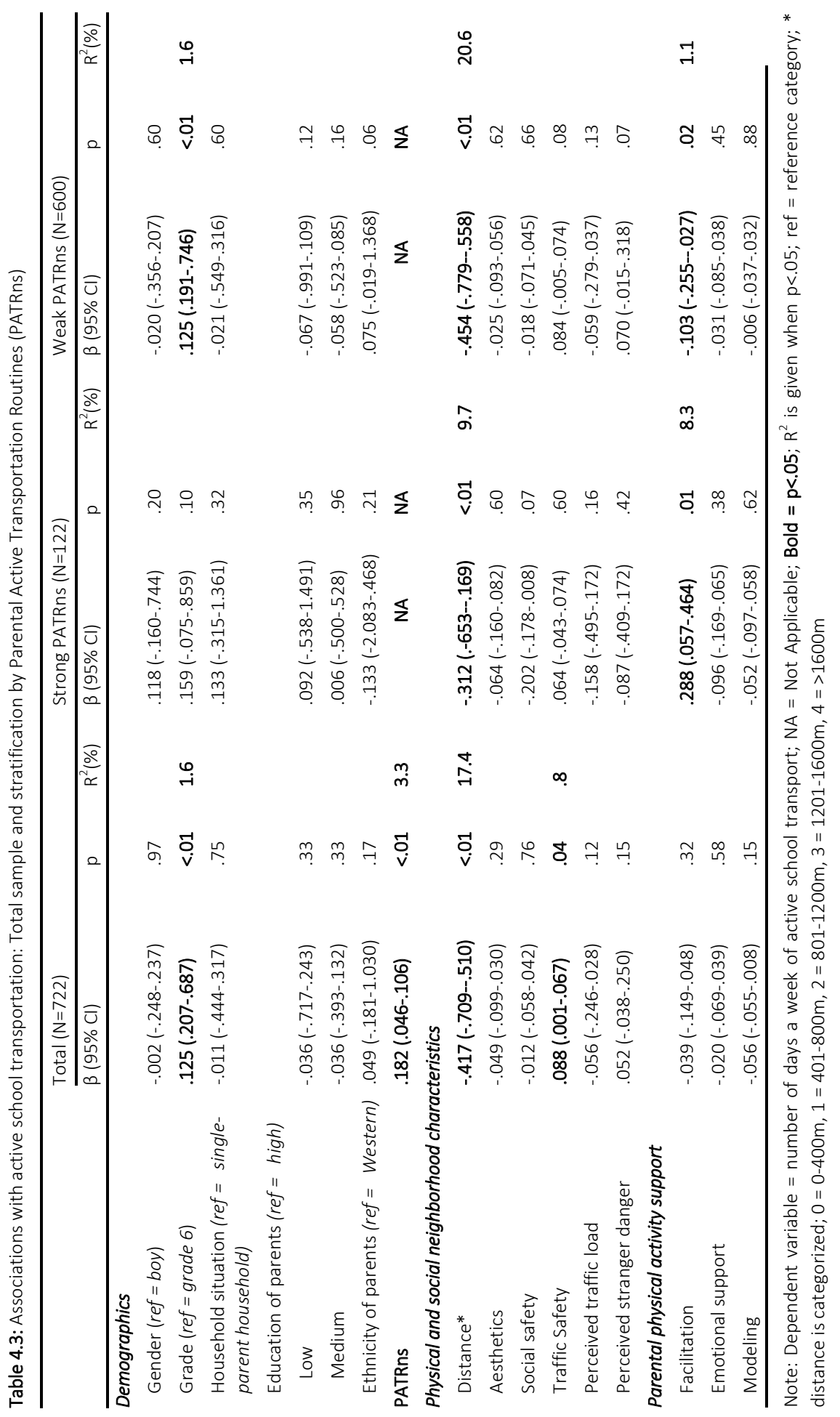




\section{DISCUSSION}

The purpose of this study was to explore the associations between Parental Active Transportation Routines (PATRns) and children's use of active school transportation. In addition, we aimed to examine the role of PATRns as a moderator of the association between the environment (physical and social neighborhood factors as well as parental support) and active school transportation. We found that PATRns were positively associated with active school transportation. When parents were more inclined to use active transportation as a routine behavior, their children more often used active transportation to school. Furthermore, PATRns were found to moderate the association between both facilitation and perceived stranger danger with active school transportation.

The direct positive association between PATRns and the use of active transportation supports the suggestion that the PATRns are an important concept in explaining children's use of active school transport. By incorporating the use of active transportation in parents' daily life patterns, irrespective of the contextual use of their active transportation (e.g., traveling to school, shops, sport clubs), children might more often travel actively to school, and potential physical and social environment barriers might become less decisive.

Interestingly, PATRns was found to moderate the association between parental physical activity facilitation and active school transportation, whereas no association was found for this factor in the total sample. The stratified analysis indicated that children of facilitating parents with strong PATRns walk or cycle to school more often. The opposite association was found for parental physical activity facilitation among parents who have weak PATRns. Jago and colleagues (2014) also failed to find an association between facilitation and active travel, in line with our results in the total sample. Our stratified results however reject Jago's assumption that facilitation for participation in physical activity does not influence active transportation (Jago et al., 2014). Our results imply that the facilitation will only positively affect active school transport in children that live in families with strong PATRns. Parents in the strong PATRns group are inclined to use active transportation as the mode of travel, including taking their child to a sports club (as facilitation is operationalized as providing logistics support for participation in physical activity). Using active transportation as a means of facilitating the child could thus be considered positive parental modeling behavior, and could also serve as an opportunity for children to actively develop traffic-related skills, such as dealing with traffic regulations. In contrast, parents in the weak PATRns group provide an inactive transportation family climate, resulting in an opposite association.

Another significant interaction by PATRns was found for the association between stranger danger and active school transportation. Several other studies identified stranger danger as major barrier for active transportation (Ahlport et al., 2008; Carver et al., 2008b). Although non-significant, our stratified analyses showed the same direction of the association for children in the weak PATRns group. For the strong 
PATRns group the opposite, also non-significant, association was found. This result was quite unexpected, based on evidence from previous studies. It is possible that by performing routine behavior, i.e. having strong PATRns, potential barriers become less dominant in explaining active school transportation, which could be the result of an exposure effect. When active transportation behavior becomes more familiar in a particular environment barriers, such as stranger danger, might become less dominant. It is also possible that parents with strong PATRns are more familiar with their neighborhood, and are thus less concerned about stranger danger. These suggestions are in line with a previous study (Salmon, Salmon, Crawford, Hume, \& Timperio, 2007) who found consistently lower perceived barriers for active transportation for children that lived closer to school.

In this study distance to school was the strongest correlate of active school transportation in all analyses. This finding is in line with most other studies assessing the effect of distance on active transportation, as distance has generally been indicated to be a strong correlate of active transportation (McDonald, 2007, 2008; Oliver et al., 2014; Panter et al., 2008; Trapp et al., 2012). We hypothesized that influences of potentially hindering physical environmental characteristics are smaller for children of parents with strong PATRns, such as distance. Note that the association (i.e. standardized beta) between distance and active school transportation was substantially lower in the strong PATRns group than in the weak PATRns group. The moderation test however was non-significant indicating no statistically significant difference in association between both groups. This non-significant interaction could be partly caused by to the unequal distribution of sample size in the two strata (strong PATRns, $\mathrm{N}=122$ versus weak PATRns, $\mathrm{N}=600$ ). Prompted by the finding that the strong PATRns group in our sample lived significantly closer to school than did children in the weak PATRns group, we performed additional sensitivity analyses for children living within 800 meters of school ( $\mathrm{N}=389)$. In this subsample a significant interaction between PATRns and distance was found in the explanation of active school transportation $(\beta=$ .730, $p=.05)$. For children in the strong PATRns group distance was not associated active school transportation ( $\beta=-.041$, ns), whereas distance was the strongest negative correlate of active school transportation in the weak PATRns group $(\beta=-.234 ; p<.01)$. This finding is in line with our hypothesis that environmental barriers may become less dominant for children whose parents have strong PATRns.

We advocate the focus on interactions between physical and social environmental characteristics in the investigation of environmental influences on physical activity in children (Gubbels et al., 2014; Gubbels et al., 2011). Pont and colleagues (2013) recommended an integrated approach to stimulate active school transportation. Our study supports this view by illustrating that insight in the interplay of parental practices and routines with other (physical and social) environmental factors will increase our understanding of determinants of active school transportation more than remaining focused on separated or isolated effects of environmental characteristics. Moreover, 
this study showed that parental practices seem to positively influence children's behavior and strong PATRns have the potential to eliminate environmental barriers. Therefore it would be recommendable to include parental components in interventions focusing on increasing children's active transportation.

This study had a number of strengths and limitations. A strong point of the study was the study sample. In total, over 700 children and their parents, distributed over 21 neighborhoods, filled in the questionnaires. In addition, we were able to observe environmental features and objectively calculate the distance of the shortest route from home to school for each individual child. A weakness of our study was that the number of days on which children used active school transportation was self-reported, which could have led to an overestimation due to social desirability. In addition, we used cross-sectional data, which only allowed us to study associations, rather than predictors of active school transportation. Note that stratified analyses were conducted with two non-equal groups, due to the theory-based classification of PATRns. Based on the dimensions in the PATRns scale, a content-based cut-off was preferred to a datadriven cut-off (e.g. using median split). This content-based cut-off enables comparability across studies, and this procedure ensured the valid formation of a strong PATRns group. In any case, secondary analyses applying a median split resulted in outcomes similar to those presented here. The smaller size of the strong PATRns group when applying the content-based cut-off could, however, have influenced the significance level of the results (relative to the weak PATRns group) in stratified analyses.

\section{CONCLUSIONS}

The current study emphasizes the importance of the concept of Parental Active Transportation Routines (PATRns). PATRns were associated with active school transportation and were found to be a moderator of the association between facilitation and active school transport and between perceived stranger danger and active school transport. Our findings indicate that the social environment in which a child operates should be taken into account when studying determinants of active transportation use. In previous research, environmental correlates of children's use of active transportation might have been biased by not including the habitual context in which the association was studied. Including parental routines into research and exploring interaction effects with environmental characteristics, could provide more insight in determinants underlying the use of active school transportation. For health practitioners the impact of parental habits and routines on children's behavior should be acknowledged in interventions to promote active school transportation resulting in an integrated approach to stimulate active school transportation. 



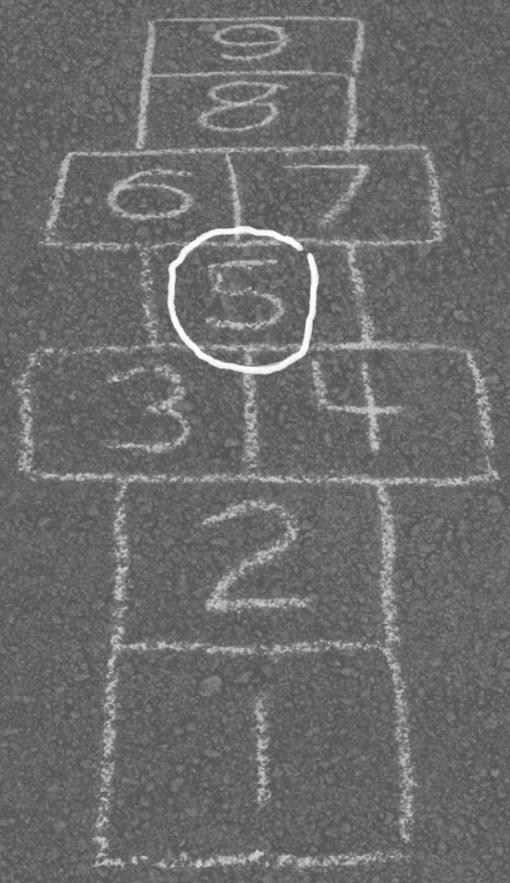

Chapter 5

Playability of School-Environments and

After-School Physical Activity among

8-11 year-old Children:

specificity of time and place

The chapter has been published as: Remmers, T., Van Kann, D., Thijs, C., de Vries, S. I. \& Kremers, S. P. J. (2016). Playability of School-Environments and After-School Physical Activity among 8-11 year-old Children: specificity of time and place. International Journal of Behavioral Nutrition and Physical Activity, 13:82. 


\section{ABSTRACT}

Introduction: Physical Activity (PA) occurs in several behavioral domains (e.g., sports, active transport), and is affected by distinct environmental factors. By filtering objective PA using children's school schedules, daily PA can be separated into more conceptually meaningful domains. We used an ecological design to investigate associations between "playability" of 21 school-environments and children's objectively measured afterschool PA. We also examined to what extent distinct time-periods after-school and the distance from children's residence to their school influenced this association.

Methods: PA was measured in 587 8-11 year-old children by accelerometers, and separated in four two-hour time-periods after-school. For each school-environment, standardized playability-scores were calculated based on standardized audits within 800 meters network buffers around each school. Schools and children's residences were geocoded, and we classified each child to be residing in $400,800,1600$, or $>1600$ meters crow-fly buffers from their school. The influence of network-distance buffers was also examined using the same approach.

Results: Playability was associated with light PA and moderate-to-vigorous PA afterschool, especially in the time-period directly after-school and among children who lived within 800 meters from their school. Playability explained approximately $30 \%$ of the after-school PA variance between schools. Greater distance from children's residence to their school weakened the association between playability of the school-environments and after-school PA.

Conclusions: This study demonstrated that relationships between the conceptually matched physical environment and PA can be revealed and made plausible with increasing specificity in time and distance. 


\section{INTRODUCTION}

The short- and long-term benefits of physical activity (PA) in children are well known. The role of attributes of the physical environment in regulation of children's PA behavior has been given increasing attention in recent years, but results so far have been mixed (Ding, Sallis, Kerr, Lee, \& Rosenberg, 2011). Although the use of objective measurements is preferred in PA-related research involving children (e.g., by accelerometers), investigating relationships between PA and the physical environment using objective measurements proves to be challenging (Ding \& Gebel, 2012).

A first challenge is assessing children's exposure to detailed elements of the physical environment. Researchers in the disciplines of health sciences, urban planning, and leisure studies all contribute to the development of measurements assessing these environmental elements (Sallis, 2009). In general, three types of measurements can be identified; self-administered surveys, systematically completed audits, and GIS-based measures (Brownson, Hoehner, Day, Forsyth, \& Sallis, 2009). In terms of objective measurements, GIS-based measures may currently be more suitable for assessing design-related features of neighborhoods on a larger geographic scale. Audits, in turn, may be more suitable to assess qualities of environmental elements in smaller-scaled environmental settings (Brownson et al., 2009; Tucker et al., 2009). In studies investigating PA in children, audits may thus be favorable in detecting (quality of) smallscaled environmental opportunities that may potentially influence leisure time PA (e.g., attractiveness and quality of public spaces or playgrounds). Recently, an instrument assessing detailed playground characteristics using systematic in-person audits of environments have been introduced as a "playability index" (Frank et al., 2012). This index stems from the Environmental Assessment of Public Recreational Spaces (EAPRS) (Saelens et al., 2006) and assesses qualities of playground-features such as facilities, aesthetics, proximity, and accessibility.

A second challenge when investigating relationships between PA and the physical environment stems from the paradigm that PA occurs in several conceptual domains (i.e., leisure, school, transport and home) (Giles-Corti, Timperio, Bull, \& Pikora, 2005; Klinker et al., 2014a; Saelens \& Handy, 2008). Investigating associations between the environment and overall PA may lead to inconsistencies, as different PA domains are regulated by distinct environmental factors (Brownson et al., 2009). An example of domain-specificity relates to children's school schedule, which largely limits their spatial freedom- and thus environmental exposure during weekdays. Separately investigating after-school PA (ASPA) helps to increase our understanding children's context-specific PA and its environmental attributes (Bauman et al., 2012; Ding \& Gebel, 2012; GilesCorti et al., 2005; Stanley, Ridley, \& Dollman, 2012; Timperio, Reid, \& Veitch, 2015). Studies using subjective measures of ASPA generally reported that boys seemed to be more active after school than girls (Leslie, Kremer, Toumbourou, \& Williams, 2010; Pate et al., 1997) and suggested a negative influence of technology-related sedentary 
activities on ASPA (Atkin, Gorely, Biddle, Marshall, \& Cameron, 2008; Pate et al., 1997). The studies that used objective measures generally indicated that ASPA contributed considerably to total PA, that boys were indeed more active after school (Arundell et al., 2013; Beighle, Morgan, Le Masurier, \& Pangrazi, 2006; Cooper et al., 2010; Dowda et al., 2007; Fairclough, Beighle, Erwin, \& Ridgers, 2012a; Hager, 2006; Hubbard et al., 2016; Mota, Santos, Guerra, Ribeiro, \& Duarte, 2003; Scott, Evenson, Cohen, \& Cox, 2007; Timperio et al., 2008; Tudor-Locke, Lee, Morgan, Beighle, \& Pangrazi, 2006). More specifically, one study reported that children do not compensate inactive days at school by increasing ASPA on a weekly basis (Dale, Corbin, \& Dale, 2000). Three studies reported on relationships between ASPA and objectively audited features of the environment (Cooper et al., 2010; Scott et al., 2007; Timperio et al., 2008). Results generally revealed that time outside resulted in 2-3 fold higher ASPA (Cooper et al., 2010), but no associations were found between the number and proximity of PAfacilities / public open spaces / playgrounds in the environment (and their specific features such as lightning and trees) and ASPA in children (Hubbard et al., 2016; Timperio et al., 2008). However in the audits of the studies above, no information was recorded about the quality of these PA-facilities (e.g., attractiveness, maintenance status or age-appropriateness). This may be important, as other factors than actual distance to public open spaces may determine the use of public open spaces or playgrounds (Scott et al., 2007; Veitch, Salmon, \& Ball, 2008).

An advantage of investigating ASPA is that when using exact school bell-times, relationships between ASPA and attributes of the school-environment can be investigated with an equal starting-point; both regarding time-opportunities and geographical location for all children attending the same school. To even further improve our understanding of this association, the ASPA time-period may be separated into even more precise time-segments after school bell-times. For example, by theory, children are all optimally exposed to the school-environment directly after school ends (i.e., bell times) but to a lesser extent later in the afternoon. Greater distances of a child's residence to the school-environment may attenuate relationships between playability and ASPA, because children living further away may be more likely to engage in ASPA at places outside the school-environment under study.

Consequently, the present study used an ecological approach to investigate the association between environmental playability and objectively measured ASPA of 8-11 year-old children, using audits of school-environments. In addition, we aimed to demonstrate that with increasing specificity in time and distance, relationships between school-environments and ASPA can be revealed and made plausible. 


\section{METHODS}

This investigation was embedded in a prospective study in the Southeast part of the Netherlands, focusing on environmental attributes and PA in Dutch primary school children. The design and protocol are described in detail elsewhere (Van Kann, Jansen, De Vries, De Vries, \& Kremers, 2015a). After obtaining parental informed consent, 815 sixth and seventh grade primary-school pupils from 21 schools participated in PA measurements and questionnaires for both one of the parents and child. Data collection took place between the $26^{\text {th }}$ of September and the $1^{\text {st }}$ of December 2012 and analyses were performed in 2015. The Medical Ethics Committee of the Maastricht University Medical Center approved this study (reference number METC 12-4-077).

\section{Measurements}

\section{After-School Physical Activity}

ASPA was measured using ActiGraph GT3X+ accelerometers $(30 \mathrm{~Hz})$ for five consecutive days (ActiGraph, Pensacola, FL), defining non-wear periods according to 60 minutes of consecutive zero's according to Troiano's criteria (Troiano, 2007). Activity intensity classification was based on Evenson's cutpoints (Evenson et al., 2008). Participants were instructed to only remove the accelerometer in water-related activities, so we explicitly instructed them to keep wearing them during sports-activities. We excluded measurements containing less than 250 minutes per day of registration time, for at least two weekdays. Although studies investigating whole-day PA patterns usually apply more stringent criteria for these registration times (Trost, Pate, Freedson, Sallis, \& Taylor, 2000), we were only interested in a smaller part of the daily PA pattern and therefore required less registration time. Weekend days and Wednesdays (because of a shortened school-schedule) were excluded. Accelerometry was aggregated to hourly averages, for each day of measurement. Using weartime-filters, ASPA was filtered from total PA registration time, based on exact school's bell times. ASPA was then separated in four two-hour time periods: 1) directly after-school-16:00, 2) 16:00-18:00, 3) 18:0020:00, and 4) 20:00-22:00. All schools ended between 14:45 and 15:30. To ensure that these time-periods represented hourly patterns, and are thus not influenced by spurious PA-spikes in children with limited period-specific weartimes, we only included accelerometer data that consisted of at least $50 \%$ of the period-specific registration time (i.e., at least one hour in a two-hour registration period) (Arundell et al., 2013; Cooper et al., 2010; Ridgers, Timperio, Crawford, \& Salmon, 2011). For the first time period, we tailored the percentage of period-specific weartime based on individual school bell-times.

Based on data from the Royal Dutch Meteorological Institute (KNMI), we also identified meteorological circumstances (i.e., average temperature, average duration of rainfall, and average duration of sunshine per day) during measurement-days. 


\section{Playability}

Playability of the school-environments was assessed by two trained researchers using the SPACE observation instrument (Aarts, de Vries, Van Oers, \& Schuit, 2012; De Vries et al., 2010), within an 800 meters radius from each school, while acknowledging natural barriers such as highways or canals. This 54-item instrument audits PA friendliness of neighborhoods and assesses characteristics such as residential density, playground characteristics, and traffic intensity, based on the Neighborhood Environment Walkability Scale but modified to reflect the Dutch environmental context (Rosenberg et al., 2009; Saelens et al., 2003). Inter-rater agreement between the two researchers who audited school-environments was acceptable (Kappa $=0.73)$. Playability was operationalized by first extracting items representing characteristics of playgrounds (excluding schoolyards) within the 800 meters crow-fly surface areas. Extracted were the playground's size in squared meters, accessibility (safely accessible versus not-safely accessible), opening hours (unlimited versus limited), maintenancestatus (poor versus good), number of facilities (e.g., climbing-facilities and soccer goals), and age-appropriateness of these facilities for 8-11 year-old children (none, partly, and fully age-appropriate). Each individual playground-characteristic was summed and standardized based on equal weights, to reflect one standardized score for each individual playground. Subsequently, these scores were aggregated to a playability index-score for each school-environment.

\section{Distance from children's residence to their school}

Since in the Netherlands a limited number of primary schools generally cover a small residential area, parent's decisions regarding the school of their children is often based on the (close) distance from their residence (Bekkers et al., 2012; Herweijer \& Vogels, 2004). Because of this vicinity to their school, children from the same school share large parts of their physical environment (Figure 5.1). Therefore, these shared schoolenvironments provide unique opportunities for investigating relationships between ASPA and the physical environment. Location of schools and respondent's residences were geocoded, and we computed a 400, 800 and 1600 meters crow-fly buffer around each school using ArcGIS (ESRI ArcGis Desktop 10.2. Redlands, CA). We subsequently classified each residence to be located 1 ) inside the 400 meters buffer-area, 2) outside 400, but inside 800 meters, 3) outside 800, but inside 1600 meters, 4) outside the 1600 meters buffer area. As crow-fly distances may be misleading because of barriers in the environment (e.g., highways or canals), we also computed network-distance as the shortest network distance in meters via the street network from each child's residence to their school using Google Maps (GoogleMaps, 2015), and recoded distances in four categories. In order to keep sample sizes within categories comparable with the crowfly distance, we based categorization on equal frequency distributions. 


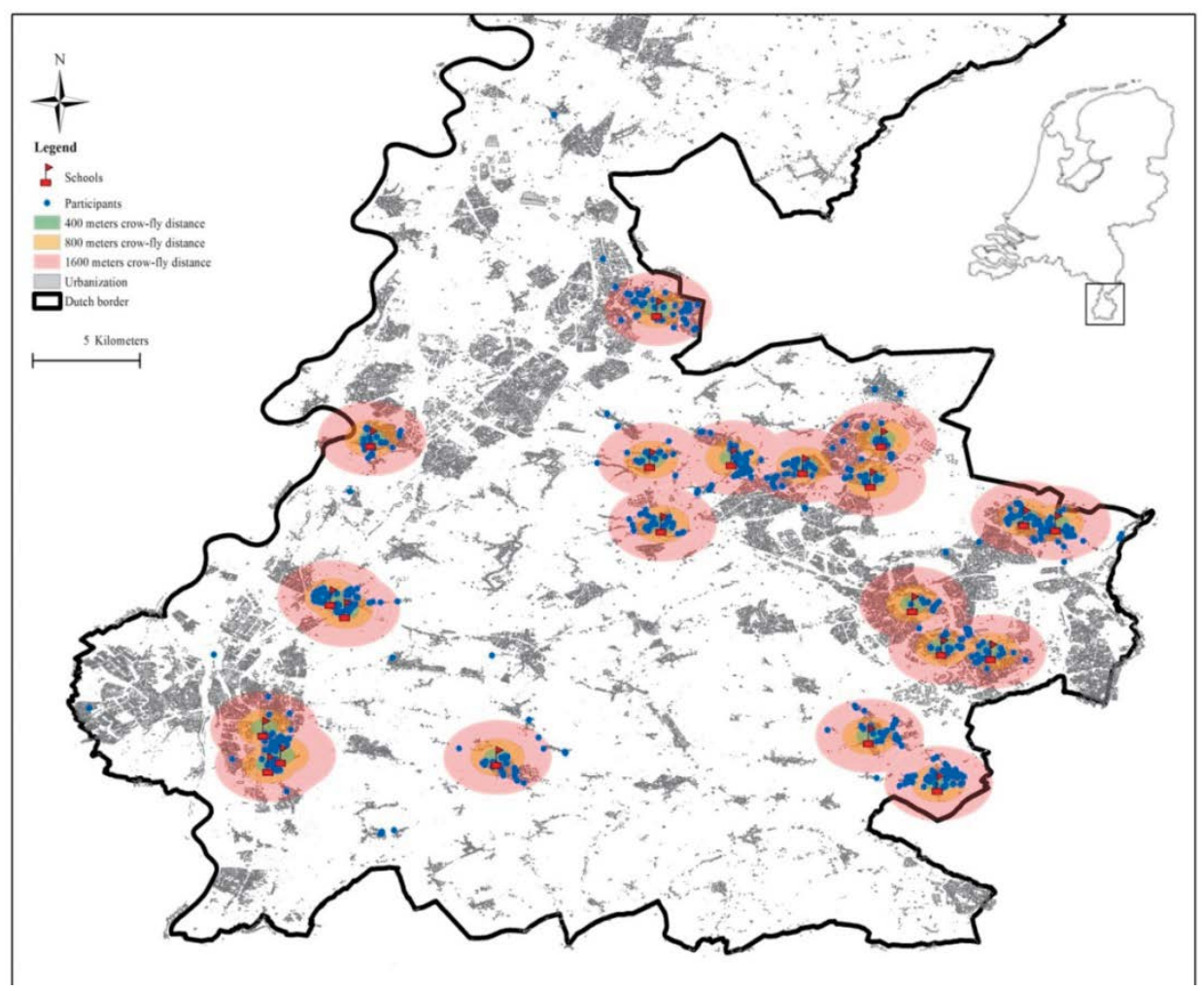

Figure 5.1: Geographical location of included schools and participants

\section{Statistical analyses}

Our analyses were performed using SPSS 20.0 for Windows (IBM SPSS Inc., Armonk, NY), and $p<0.05$ indicated statistical significance. Our dependent variable was the ASPA performed in light and moderate to vigorous intensity for each hourly time-interval of measurement. Our primary independent variable was the combined playability index of the different school-environments. We first described the percentages of light PA (LPA) and moderate-to-vigorous PA (MVPA) across four two-hour time-periods after school, and across four distance-buffers from schools using univariate analyses of variance (Table 5.1).

We performed multilevel linear mixed models in order to account for the timedependent structure of the data. We specified a random intercept and slope for the hourly time-intervals, nested within the specific dates at which a respondent's accelerometry commenced. Analyses were also adjusted for hourly weartime, average daily temperature, daily duration of rainfall, and daily duration of sunshine per day. We evaluated whether age and gender moderated the association between playability and ASPA, but as we did not find moderation, we only adjusted for age and gender. 
To investigate the influence of the four time-periods and distance-categories on the relationship between ASPA and playability, we entered the appropriate interaction terms in our linear mixed models. Using dummy-coded interaction-terms between distance-categories and the playability index-score, we were able to estimate main effects of playability for each of the two-hour time periods, while still acknowledging the time-dependent structure with the random intercept and slope. Distance-categories were both conceptualized as crow-fly and network-distances. Finally, we also repeated our analyses now stratifying for the time-periods and distance-categories simultaneously to investigate their interactive influence (Table 5.4).

\section{RESULTS}

In total, 587 children (74.2\%) provided valid ASPA measurements, for two (44.8\%) and three (55.2\%) valid weekdays, respectively. The 280 participating boys and 307 girls were aged 10.2 years on average (range 8 to 11 years) (Table 5.1). Across all time-periods, $27.9 \%$ of the time after-school was spent in LPA, which accumulated to 103.4 minutes per day (SD=23.9). MVPA accounted for $7.7 \%$ of the after-school time, accumulating to 28.4 minutes per day ( $S D=14.6)$. Daily percentages of LPA declined across the four timeperiods, while MVPA slightly declined after 16:00, but increased again after 18:00. Daily percentages of LPA and MVPA were comparable across the distance-categories (no statistically significant differences in analysis of variance, data not shown).

\section{Playability and after-school Physical Activity by time-period}

Children who attended schools in areas with higher playability scores, generally showed significantly higher LPA and MVPA in the first two time-periods (i.e., between the end of the school day and 18:00) (Table 5.2). By contrast, in the subsequent time-periods, the association between playability and ASPA attenuated. Only the relatively small amount of time performed in light intensity between 20:00 and 22:00 unexpectedly showed a statistical significant relationship with playability.

\section{Playability and after-school Physical Activity by distance-categories}

Only if children lived within 400 meters from their school, after school LPA was positively associated with playability (Table 5.3). When children lived beyond 400 meters from their school, associations attenuated and were no longer statistically significant. For after school MVPA, the attenuating influence of distance from home to school was detectable when exceeding the $800 \mathrm{~m}$ crow-fly or network-distance.

To examine the interactive influence of time and distance, analyses of time-periods were stratified for distance-categories from home to school (Table 5.4). The results of 
these stratified analyses were generally in line with the results described above. Regarding LPA, the attenuating influence of time-periods and distance-categories was comparable to the results Table 5.2 and 5.3, except for the distance greater than 1600 meters and time-period after 20:00 hours. Regarding MVPA, attenuating influences of time and distance were also comparable with Table 5.2 and 5.3, but in some instances revealed deeper insights. For example, ASPA after 16:00 hours (i.e., second time-period) was only associated with playability in children that lived within 400 meters from their school. When these analyses were repeated using network-distance, similar attenuating influences of time and distance were found.

Table 5.1: Descriptive statistics of the study population

\begin{tabular}{|c|c|}
\hline Individual level $(n=587)$ & \\
\hline Age; mean years $(\mathrm{sd})$ (missing $n=10$ ) & $10.2(0.7)$ \\
\hline Gender; $n$ boys (\%) & $280(47.7)$ \\
\hline Ethnicity; $n$ Dutch (\%) (missing $n=9$ ) & $490(84.4)$ \\
\hline \multicolumn{2}{|l|}{ Crow-fly distance from home to school; $n(\%)$} \\
\hline within 400 meters & $187(31.9)$ \\
\hline within 800 meters & $225(38.3)$ \\
\hline within 1600 meters & $119(20.3)$ \\
\hline outside 1600 meters & $56(9.5)$ \\
\hline \multicolumn{2}{|l|}{ Network distance from home to school; $n(\%)$} \\
\hline distance $\leq 499$ meters & $138(23.5)$ \\
\hline distance 500 - 799 meters & $137(23.3)$ \\
\hline distance 800 - 1199 meters & $171(29.1)$ \\
\hline distance $\geq 1200$ meters & $141(24.0)$ \\
\hline Light PA by time of the day; mean \% of time per day (sd) & $27.9(6.6)$ \\
\hline end of the school day - 16:00 hours; mean \% (sd) $(n=539)$ & $35.3(8.8)$ \\
\hline $16: 00$ - 18:00 hours; mean \% (sd) $(n=586)$ & $29.2(8.2)$ \\
\hline 18:00 - 20:00 hours; mean \% (sd) $(n=585)$ & $27.5(7.7)$ \\
\hline $20: 00-22: 00$ hours; mean $\%(s d)(n=326)$ & $20.8(9.5)$ \\
\hline MVPA by time of the day; mean \% of time per day (sd) & $7.7(4.0)$ \\
\hline end of the school day - 16:00 hours; mean \% (sd) $(n=539)$ & $10.4(6.2)$ \\
\hline $16: 00-18: 00$ hours; mean \% (sd) $(n=586)$ & $6.8(4.8)$ \\
\hline 18:00 - 20:00 hours; mean \% (sd) $(n=585)$ & $9.2(7.5)$ \\
\hline 20:00 - 22:00 hours; mean \% (sd) $(n=326)$ & $4.8(6.2)$ \\
\hline Light PA by crow-fly distance; mean \% of time per day (sd) & $27.9(6.6)$ \\
\hline within 400 meters $(n=187)$ & $28.2(7.0)$ \\
\hline within 800 meters $(n=225)$ & $28.0(6.4)$ \\
\hline within 1600 meters $(n=119)$ & $27.9(6.3)$ \\
\hline outside 1600 meters $(n=56)$ & $27.3(6.5)$ \\
\hline MVPA by crow-fly distance; mean \% of time per day (sd) & $7.7(4.0)$ \\
\hline within 400 meters $(n=187)$ & $7.9(4.1)$ \\
\hline within 800 meters $(n=225)$ & $7.9(4.1)$ \\
\hline within 1600 meters $(n=119)$ & $7.4(4.0)$ \\
\hline outside 1600 meters $(n=56)$ & $7.3(3.8)$ \\
\hline
\end{tabular}


Table 5.2: Associations between playability and after-school physical activity intensities by time of the day

\begin{tabular}{lll}
\hline & \% Light PA & \% MVPA \\
\hline Time of the day & & \\
End of the school day $-16: 00$ hours $(n=539)$ & $0.043(0.026$ to 0.060$)$ & $0.029(0.008$ to 0.048$)$ \\
16:00 - 18:00 hours $(n=586)$ & $0.018(0.002$ to 0.034$)$ & $0.028(0.012$ to 0.045$)$ \\
18:00 - 20:00 hours $(n=585)$ & $0.001(-0.015$ to 0.018$)$ & $-0.007(-0.024$ to 0.010$)$ \\
20:00 - 22:00 hours $(n=326)$ & $0.052(0.032$ to 0.072$)$ & $0.016(-0.005$ to 0.038$)$ \\
\hline
\end{tabular}

Standardized beta's (with 95\% confidence intervals in brackets) from linear mixed model analyses with a random intercept and slope over time (one-hour periods), nested within the dates at which measurement commenced. Results were adjusted for age, gender, average temperature, average duration of rainfall, and average duration of sunshine per day. Bold number represents statistical significance at $p<0.05$. 


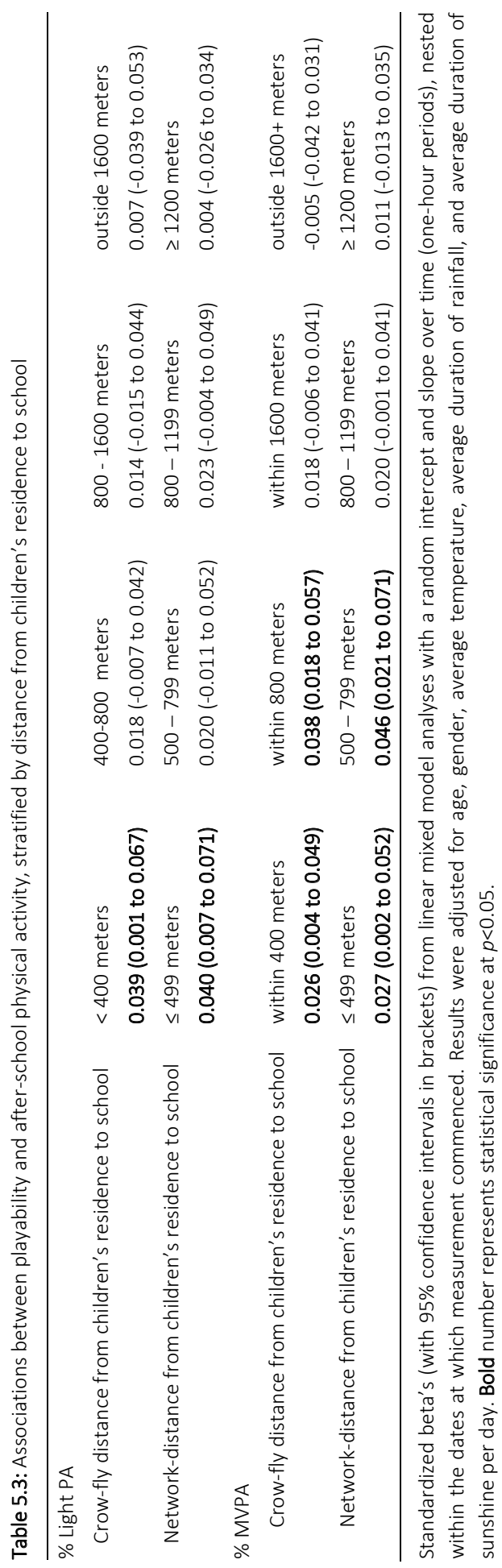




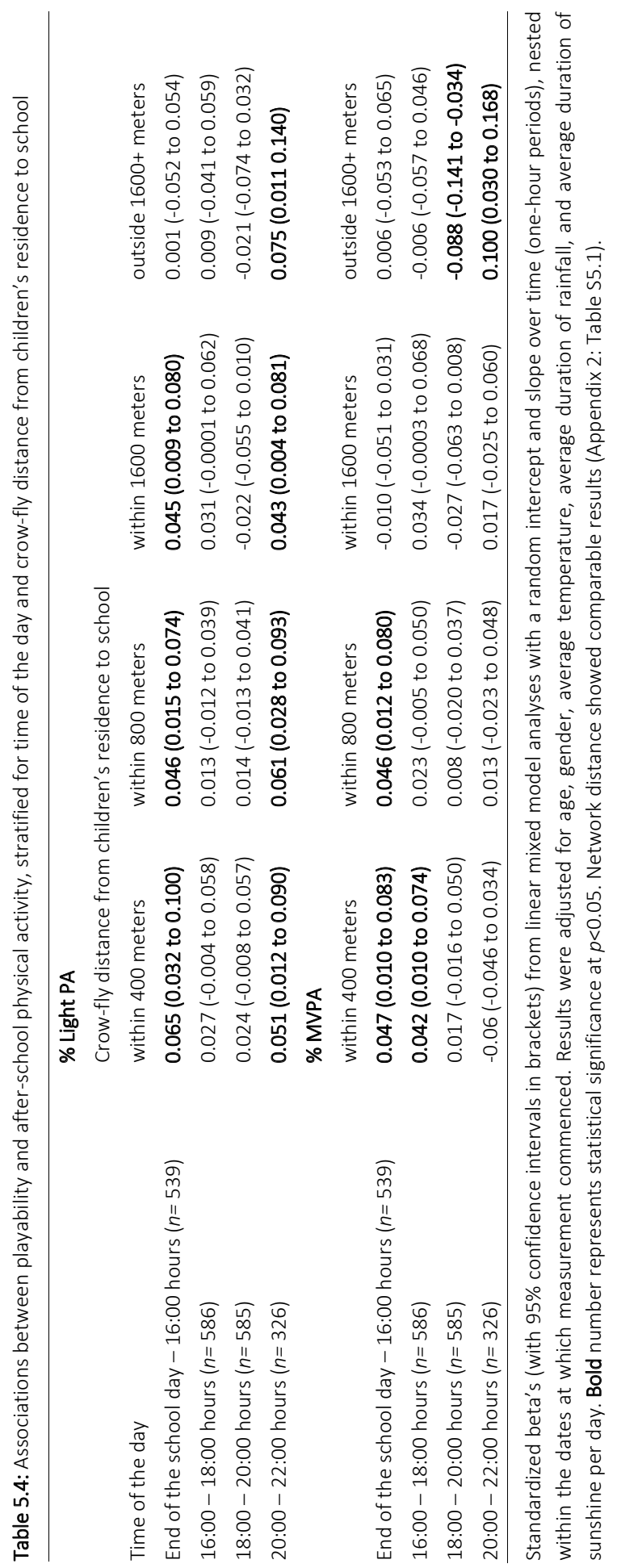




\section{DISCUSSION}

This study investigated the association between playability of school-environments and ASPA, separately for time-periods within the after-school period and distancecategories from school to children's residence. We demonstrated that the influence of playability is highly dependent on these time-periods and distance from home to their school: greater distance attenuated the influence of playability of the schoolenvironment on ASPA, especially in the first hours after-school. As expected, children that lived outside the study-area for which playability was audited generally showed no relationships between playability of the school-environment and ASPA.

When comparing our findings in the light of other studies that have investigated ASPA objectively, we can confirm that boys were more active after-school compared to girls (Beighle et al., 2006; Hubbard et al., 2016; Tudor-Locke et al., 2006). In contrast to the study of Mota et al., which reported that boys were more active in the later timeperiods after school (Mota et al., 2003), we found that the difference between boys and girls was stable across the first three time-segments, and this difference decreased at later periods in the evenings (data not presented). As this study did not compare PA during school hours with ASPA, we cannot compare our results with studies that indicated that ASPA significantly contributed to total PA (Beighle et al., 2006; Fairclough et al., 2012a; Tudor-Locke et al., 2006). Although Timperio et al. found that relationships between ASPA and individual features of public open spaces were different for boys than for girls (Timperio et al., 2008), we found no such moderation mechanisms in our playability index. This may be because in our study, potential gender differences may annul at a higher level of abstraction when utilizing a standardized index-score of playground qualities instead of individual features of public open spaces. Further research is however needed to clarify potential gender-related moderation mechanisms. In addition, Scott et al. argued that perceptions of easy access and the number of PA-facilities, but not objectively determined number and proximity of PAfacilities were related to adolescent girls' non-school PA (Scott et al., 2007). Although we acknowledge the importance of perceived accessibility and/or presence of environmental attributes in PA-research, we cautiously suggest that this played a relatively minor role in our study because our playability index aggregated qualities of multiple playgrounds, accounting for accessibility and the number of these playgrounds in the school-environment.

When taking into account the relatively small strata-specific sample sizes at greater distance-categories, relationships may seem relatively weak. However, this study demonstrated that relevant relationships between physical environments and ASPA can be revealed and made plausible, with increasing specificity in time and distance. This demonstrates that loss of statistical power due to lower number of observations is compensated by increased discriminative precision thanks to time-place specificity. 
Our proposed playability score allowed for aggregation of playground characteristics of the school-environment within multiple geographic settings. The SPACE observation instrument is comparable to the Neighborhood Environment Walkability Index in terms of identified factors/scales (e.g., facilities, aesthetics, proximity, accessibility), aggregation procedure (computation of means from subscale items), and normalization procedures (Rosenberg et al., 2009; Saelens et al., 2003). The concept of playability was introduced in the studies of Frank and Roberts (Frank et al., 2012; Roberts, Ray, Biles, Knight, \& Saelens, 2015). As the study of Roberts solely reported on a protocol of developing a playability-index, to date no direct comparisons can be made with the current study's SPACE observation instrument. The study of Frank et al. (Frank et al., 2012) derived the quantity and quality of public parks using the Environmental Assessment of Public Recreational Spaces (EAPRS). With the exception of the qualityconcept 'shade' and trails, all concepts and methodology of EAPRS (i.e., independent audits of two trained observers) were also represented in the current study.

One may argue that our results are not influenced by differences in playability, but by differences between schools in active transport. However, we found no indication for an ASPA-increase in children who lived more than 800 meters from their school at later time-periods (potentially to compensate for motorized transport). In addition, children reported the number of days per week they walked or cycled to school and the mean duration of those trips, and sensitivity analyses revealed that additional adjusting for active transport did not alter our results (data not shown). As our sample size did not allow further segregation for active transport use, we recommend future research to address the influence of active transport in this relationship.

Our time-specific analyses showed that LPA performed after 20:00 hours was significantly related to playability. This was unexpected because in the Netherlands, during this time of the year it was dark. As we had no diaries, we were unable to confirm to what extent and where children were active at that time. Apart from the possibility that relationships in the late evening may be influenced by the relatively small sample sizes because of non-weartime periods, we can only speculate that this behavior may be merely related to LPA inside their houses, or that potential differences (by chance) between schools in bed-times may have influenced this association, rather than the actual influence of playability of the environment. The same explanation may suffice for unexpected statistically significant relationships between MVPA and playability in children living more than 1600 meters from their school. In addition, we observed that the MVPA percentages increased between 18:00-20:00, but strength of relationships between MVPA and playability did not increase accordingly. This potentially means that the observed increase in mean MVPA percentages was explained by other factors than playability of the environment, such as sports participation (organized forms of after-school activity often occur during evening-hours). Future studies are advised to include some diaries about sleep times and main activities after school (e.g., organized sports participation in the evenings), and are warranted to 
examine potential if attenuation of playability by organized sports participation would persist in spring, and whether breaks in organized sports (e.g., during summer recess) would relate to a stronger relationship between playability and PA after-school-time.

\section{Strengths and weaknesses}

The major strength of this study is that we attempted to improve the understanding of ASPA and a potentially plausible relationship with playability of the physical environment, by measuring time-period specific ASPA and detailed, qualitative characteristics of playgrounds in school-environments.

The present study was confined to an ecological design, assessing characteristics at the school level. Recent methodological innovations such as combined accelerometry and GPS measurements can provide opportunities for even more in-depth analyses of the association between environmental attributes and domain- specific PA. GPS measurements can for example be used to identify time spent outside (Cooper et al., 2010) or even at the schoolyard or at specific playgrounds (Hurvitz, Moudon, Kang, Saelens, \& Duncan, 2014). Moreover, integrating multiple data-sources (e.g., accelerometry, GPS, GIS, audits, school's time tables, participant diaries) into comprehensive databases provide unique opportunities for investigating PA and other health behaviors, while accounting for its spatial and temporal specificity (Goodman, Page, \& Cooper, 2014; Klinker et al., 2014a).

This study applied a threshold of $50 \%$ period-specific registration time to prevent our analyses being influenced by short- spurious spikes of (intense) PA. Although two studies also used this 50\% threshold (Arundell et al., 2013; Ridgers et al., 2012a) and one study used a 60\% threshold (Cooper et al., 2010), reliability and relative influence of exact thresholds for period-specific registration times are debatable. In addition, the definition of non-weartime periods (e.g., 60 minutes of consecutive zero's) highly influences period-specific thresholds, as it determines whether relatively short- or longer bouts of inactivity are classified as non-weartime periods. Future studies are therefore warranted to investigate the influence of period-specific thresholds in depth while also accounting for differences in non-weartime definitions; for example by comparing its influence in relationships with ASPA patterns.

One can speculate on alternative protocols in computing playability (e.g., multiplication of individual items or based on unequal weights). However, as we were unable to find an evidence-base for such alternative protocols, we decided to aggregate based on equal weights. To check for potential errors in the aggregation procedure we fed back the aggregated scores to the auditors of school-environments. Hereafter, no alterations were made to the aggregation protocol.

As our audits were limited to 800 meter buffers from participating schools, it may seem logical that the relationship between playability of the school-environment and ASPA attenuated for children that lived outside this study-area. However, the aim of the 
present study was to demonstrate the temporal and site-specific mechanisms, and thus underline that in future research investigating relationships between PA and the environment, time- and place specificity is warranted. In addition, as in the Netherlands no public primary schools have organized public transport services from school to children's homes and the majority of 8-11 children rely on active transport to get home after school, all children are likely to have at least the opportunity to be exposed to PAopportunities in their school-environment.

As data collection was conducted in autumn, our results may not be comparable with other studies that usually perform their PA measurements in spring. Future studies are therefore encouraged to replicate this methodology in spring (or expressly study modification by season). In addition, one-third of the children in our sample experienced one hour earlier sunset due to daylight savings time change in fall. Similarly to the results of Goodman et al (Goodman et al., 2014), we found that children measured in the period with earlier sunsets were less active, both in LPA and MVPA, independent of other meteorological measures (results not shown). In addition, differences in children's PA were especially noticeable in the evenings (data not shown). Because of the relatively unequal distribution of participants measured during daylight saving time versus standard time and our limited sample size, we were unable to check whether the association between playability and ASPA differed between children measured with daylight saving periods versus standard time.

\section{Impact}

We found playability to be related with ASPA only in the time-period directly afterschool, especially in children who live within 800 meters distance from their school. First, this showed that children who lived further away from school, were relatively confined to their own residential neighborhood after-school, thus making limited use of the school-environment for ASPA. Second, playgrounds in school-environments only had a limited influence on children's ASPA throughout the day, and competing PAdomains (e.g., sports participation) may have explained variability in especially MVPA percentages in later time-periods of the day.

\section{CONCLUSION}

This study demonstrated the importance of playability of school-environments as an environmental determinant of after-school PA in children. With time and space filtering, the conceptual understanding of ASPA and its association with the physical environment can be improved. This may help to develop more tailored interventions to promote specific PA-domains at specific time-periods during the day. All in all, our analytical design with time and space filtering may encourage researchers to look into 
more domain-specific parts of children's PA behavior within the opportunities and limitations of their own sample, embedded in strong theoretical foundations. 



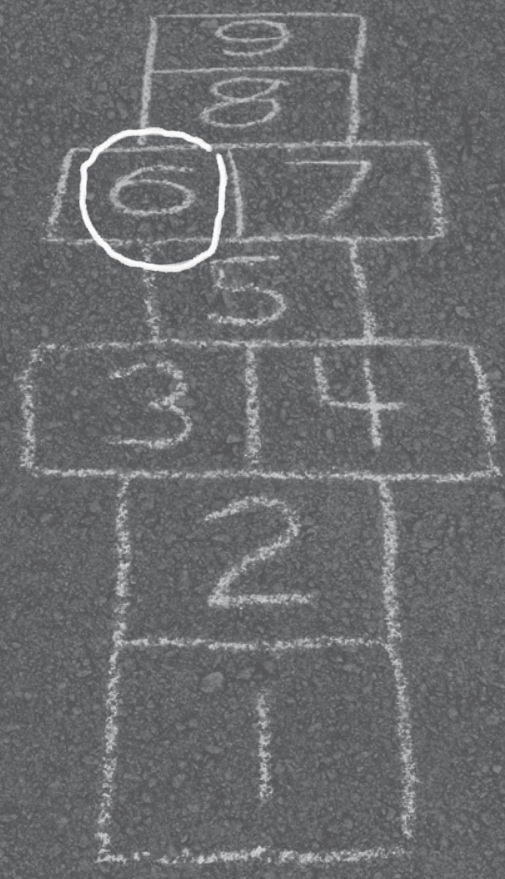

\section{Chapter 6}

\section{Schoolyard characteristics, physical activity and sedentary behavior: Combining GPS and accelerometry}

This chapter has been published as: Van Kann, D. H. H., de Vries, S. I., Schipperijn, J., de Vries, N. K., Jansen M. W. J. \& Kremers, S. P. J. (2016). Schoolyard characteristics, physical activity and sedentary behavior: Combining GPS and accelerometry. Journal of School Health, 86(12), 913-921. 


\section{ABSTRACT}

BACKGROUND: Physical activity (PA) is decreasing among children, while sedentary behavior (SB) is increasing. Schoolyards seem suitable settings to influence children's physical activity behavior. This study investigated the associations between schoolyard characteristics and moderate-to-vigorous physical activity (MVPA) and SB of children aged 8-11 years at schoolyards.

METHODS: Twenty primary schools in the Netherlands were involved. A total of 257 children wore an accelerometer and GPS device for 5 consecutive days to objectively assess their PA levels and presence at the schoolyard, respectively. Accelerometer and GPS data were merged using the personal activity and location measurement system (PALMS). Multilevel linear regression analyses were used to study correlates of MVPA and SB at schoolyards.

RESULTS: On average children spent 54 minutes a day at the schoolyard, 9 minutes of which were spent in MVPA and 20 minutes in SB. Boys engaged in MVPA longer than girls at the schoolyard. Fixed equipment, such as high bars and soccer goals, teacherinitiated activities, and the presence of a ball games policy were correlates of more MVPA and less SB.

CONCLUSION: Well-designed schoolyards, including PA-enhancing fixed equipment, a supportive PA climate created by teachers, and supportive schoolyard policies may contribute to increased physical activity and decreased sedentary behavior during school recess among school-aged children. 
Regular physical activity (PA) is important for children's health (Loprinzi et al., 2012; $W H O, 2010)$. Sufficient PA is associated with several beneficial health outcomes, (Andersen, Riddoch, Kriemler, \& Hills, 2011) increased psychosocial well-being (Biddle \& Asare, 2011) and better school performance (Singh et al., 2012). Nevertheless, children's levels of PA are decreasing (McDonald, Brown, Marchetti, \& Pedroso, 2011), while levels of sedentary behavior, such as playing video games on smartphones, inactive play in school recess and watching other children's activities, have been increasing in the last decade (Hendriksen, Bernaards, Hildebrandt, \& Hofstetter, 2013; Lou, 2014). Sedentary behavior (SB), rather than lack of PA, has been identified as an independent health threat for children (Hancox, Milne, \& Poulton, 2004; Lou, 2014; Tremblay et al., 2011). SB should be considered as independent behavior with its own determinants, independent of (determinants of) PA (Pearson et al., 2014; Saunders et al., 2014). The development of a healthy PA pattern in childhood is essential for a healthy lifestyle in later life. Sedentary patterns seem to track into adulthood even more than PA patterns, underlining the importance of encouraging both sufficient PA and limited SB in children (Jones, Hinkley, Okely, \& Salmon, 2013; Singh et al., 2008).

All children spend a substantial part of their time at school, making the school environment highly important for children's PA levels (Klinker, Schipperijn, Toftager, Kerr, \& Troelsen, 2015; Ridgers, Stratton, \& Fairclough, 2006). The school environment can contribute to daily moderate-to-vigorous physical activity (MVPA) and decrease SB by providing a supportive physical and social environment, including supportive environments for active school transport (Klinker et al., 2015; Van Sluijs et al., 2009), appropriate and sufficient physical education lessons (Lonsdale et al., 2013; Slingerland \& Borghouts, 2011; Trudeau \& Shephard, 2005), and supportive environments for PA during recess (Kriemler et al., 2011; Parrish et al., 2013).

PA during recess has been reported as a major contributor to the daily recommended amount of PA (Dessing et al., 2013; Klinker et al., 2014a; Ridgers et al., 2006). Recess time can consist of both indoor and outdoor play, with outdoor play generally being associated with higher levels of PA (Tran, Clark, \& Racette, 2013). PA levels of children during recess can be influenced by the built environment of the schoolyard (Broekhuizen et al., 2014; Escalante et al., 2014; Ridgers et al., 2012a). In previous research, several physical features of the schoolyard have been associated with PA during recess, such as playground density (number of children per square meter) (Fairclough, Ridgers, \& Welk, 2012b; Ridgers et al., 2012a) and the availability of portable and fixed play equipment (Ridgers et al., 2010b; Taylor et al., 2011). However, many questions remain unanswered, such as the direction and strength of associations between physical features and PA levels during recess, possibly influenced by contextual factors (Broekhuizen et al., 2014; Escalante et al., 2014; Pagels et al., 2014), e.g. one schoolyard feature on its own could enhance PA, whereas the same feature may not affect PA when it is provided at a schoolyard with a wide variety of other features. However, no study has previously examined the association between specific 
schoolyard characteristics and sedentary behavior. Despite lacking evidence of schoolyard characteristics in SB, the need for intervening on SB at the schoolyard gains attention, like in a recent schoolyard intervention study that outlined SB as important target behavior (Andersen et al., 2015).

One explanation for the mixed and/or unspecific results with regard to correlates of PA in schoolyards could be the quality of assessment of the schoolyard environment and the method used to assess PA levels (Jankowska, Schipperijn, \& Kerr, 2015). In general, objectively assessed PA is more accurate and sensitive than self-report methods, in which PA is often overestimated and SB is underestimated (Dyrstad, Hansen, Holme, \& Anderssen, 2014). However, objective assessment methods, e.g. using accelerometers, do not provide information about the context in which PA occurred. Scientific studies based on time periods (using the school's timetables) might find associations between children's PA levels and the schoolyard environment, whereas it is not certain whether the child was actually at the schoolyard. Combining the use of accelerometers to objectively measure PA with location information from the Global Positioning System (GPS) may prevent this possible error, making it a suitable method to evaluate the influence of the built environment on behavior (Andersen et al., 2015; Krenn, Titze, Oja, Jones, \& Ogilvie, 2011).

The aim of this study was to investigate the influence of schoolyard characteristics on MVPA and SB of children aged 8-11 years at schoolyards, using children's objectively assessed PA levels as well as their objectively GPS-defined locations. We also explored whether correlates of MVPA and SB differed for morning recess, afternoon recess and out-of-school time at the schoolyard.

\section{METHODS}

\section{Participants}

Recruitment of schools and children. Data included in this study were derived from the baseline measurement of the Active Living study, which focuses on encouraging PA in primary school children in the Southern Limburg region of the Netherlands. Twenty-one schools were recruited by employees of the Southern Limburg public health service to take part in the Active Living study. The main inclusion criterion for schools to participate in the Active Living study were 'consent by the schoolboard and the local authorities of the municipality in which the school is situated' and 'school situated in a Iow SES area'. Twenty schools were eligible to be included in this study. One school moved to a temporary building and was excluded. No intervention taking place while baseline measurements were being made. Children in grades 6 and 7 of the Dutch school system (8-11 years old) were invited to take part in the Active Living study. 
Recruitment of children took place by having the main researcher (DVK) introduce the project in the classroom and by distributing information letters to the parents.

Due to the limited availability of GPS devices, data collection took place stepwise between September 2012 and June 2013; schools were visited twice with a 6-month interval. The winter period (December 2012 - March 2013) was excluded to avoid possible seasonal effects (Oreskovic et al., 2012). In total, 1328 children were approached to take part in this study, and 811 (61.1\%) agreed to wear an accelerometer. Ethical approval (by parents) was provided prior to inclusion. A random selection of these children ( $N=257$; $32 \%$ of accelerometer wearers) also wore a GPS device.

\section{Instrumentation}

Assessment of PA levels and locations. Children were asked to wear an accelerometer (Actigraph $\mathrm{GT} 3 \mathrm{X}, 30 \mathrm{~Hz}$ ) for at least 5 consecutive days during waking hours, except during water-related activities (e.g. swimming and showering). Selected children who also wore a GPS device (QStarz, BT-Q1000XT, $15 \mathrm{sec}$ epoch) were instructed to use the same protocol as for the accelerometers, and were additionally instructed to charge the battery of the GPS device overnight.

Assessment of schoolyard characteristics. The physical environment of schoolyards was observed in August 2012 using the SPACE checklist (De Vries et al., 2010). In total, 12 types of fixed equipment (e.g. soccer goals, high bars, and playground markings; Figure 6.1) were assessed by two researchers, in terms of presence (number) and quality (1=poor; $2=$ moderate; $3=$ good). Furthermore, the presence of green spaces ( $1=$ not present/limited; $2=\geq$ substantial) and general upkeep (1=poor; 2=moderate; 3=good) of schoolyards were judged. If the researchers disagreed, they reached consensus by discussion and revisiting the schoolyards immediately after the observations. The schoolyard's size was defined in square meters using GIS-information in ArcGIS version 10.2.

Additional telephone interviews with each school's principal were conducted to assess the school's schoolyard policy. Schoolyard policies were checked for: opening hours of the schoolyard (range: $0=$ not open - 3=unlimited opening hours), use of scattered recess for classes (no/yes), number of children at the schoolyard while in (scattered) recess, role of teachers during recess, number of supervisors during recess, initiator(s) of PA activities (child only/teacher and child; included as social environment), restrictive policies on running (no/yes), policies on ball games areas (no restriction/restrictions by location), and policies on the use of the schoolyard in poor weather conditions ( 1 =stay inside; $2=$ free to choose; $3=$ go outside).

\section{Procedure}

Data processing. Accelerometer and GPS data were combined using the personal activity and location measurement system (PALMS), developed by the University of 
California, San Diego. This web-based tool aggregates both types of data to a dataset in which the child's location and corresponding PA level are represented for each 15second period (epoch). A purpose-built PostgreSQL database was developed to combine the PALMS data with GIS data, additional child data (gender and grade), and school timetable information, i.e. starting, ending and recess times of each participating class. All 20 schoolyards were digitized in ArcGIS 10.2, and a $10 \mathrm{~m}$ Euclidian buffer added to account for potential errors in GPS data (Schipperijn et al., 2014). All data points in the database that fell within the buffered schoolyards were selected for further analysis.

The PostgreSQL database was used to create a data file consisting of aggregated values for wear time, average counts per minute (CPM), time spent in MVPA (CPM > 2295), and time spent in SB (CPM < 101) per child per day (Evenson et al., 2008). These aggregated values were created for multiple periods to differentiate between freely chosen schoolyard exposure and obligatory schoolyard exposure: total day (at schoolyard), morning recess (at schoolyard) (recess 1), afternoon/lunch recess (at schoolyard) (recess 2), and outside school hours at schoolyard. The physical schoolyard characteristics and schoolyard policies were added separately to the final dataset.

Data reduction. The database produced aggregated data for five days of measurement for each child. A weekday filter was used to exclude weekend days. To be included in the analyses, children had to provide at least 1 valid weekday with a minimal wear time of 8 hours (480 minutes) a day (Jago et al., 2013). To prevent overestimation of PA due to potential reactions to the measuring equipment, the first day of wearing the devices was excluded (Mattocks et al., 2008). Furthermore, a minimal wear time of 5 minutes was defined for each of the three schoolyard periods. The use of these filters excluded the participants who only crossed the schoolyard to enter the school building. A total schoolyard visit score for a day was defined as fulfilling the wear time criterion for all three periods. Finally, all children's data were aggregated to an average score per period. An additional criterion of at least 5 children per schoolyard environment was introduced to guarantee validity of associations between schoolyard characteristics and PA behavior.

\section{Data analysis}

Aggregated data created by the PostgreSQL database were analyzed using SPSS version 20.0 (IBM Corp, NY, USA). Statistical significance was defined as $p<.05$. Period-specific schoolyard time spent on MVPA and SB, and CPM, were defined by frequency analyses; gender differences were tested by independent samples t-tests. Correlates of MVPA and SB were analyzed using a mixed linear regression model. Independent variables were physical, social, and policy-related schoolyard characteristics and child-related variables, i.e. grade and gender. A multilevel structure was used; level one was the school level, including physical, social, and policy-related schoolyard characteristics, including equipment (presence multiplied by quality score), presence of green spaces, 
quality of general upkeep, available square meters per child, initiator of schoolyard PA activities, schoolyard weather policy, schoolyard ball games area policy, and schoolyard accessibility before school hours. Level two consisted of child-related data, i.e. gender and grade. The backward deletion method was used to exclude the least significant variables $(p<.10)$. Analyses were conducted both for each schoolyard period as well as for total day schoolyard visit data.

\section{RESULTS}

\section{Demographics}

A total of 20 schools participated in this study, with 257 children (ranging from 5-24 children per school) wearing both an accelerometer and a GPS device, in accordance with the inclusion criteria. Fifty-three percent $(\mathrm{N}=137)$ of the sample were girls. Distribution across grades was almost equal; 48\% ( $N=123)$ in grade 6 vs. $52 \%(N=134)$ in grade 7 . Stratification by gender did not result in significant differences for grade distribution. The 5-minute 'at schoolyard' inclusion criterion led to a varying number of children being included in different schoolyard time periods. The average size of a schoolyard was 1912 square meters and per child 14.3 square meters were available at the schoolyard during recess (Table 6.1).

Table 6.1: Descriptive of included schoolyard characteristics

\begin{tabular}{lll}
\hline Schoolyard characteristics & Data ranges & Mean (SD) \\
\hline Physical Environment* & & $2.47(3.04)$ \\
Small soccer goal & $0-6$ & $6.00(3.97)$ \\
High bar & $0-12$ & $5.94(5.99)$ \\
Marking & $0-24$ & $1912.18(791.34)$ \\
m2 schoolyard & $724-3786$ & $14.27(4.62)$ \\
m2 per child & $8.8-22.8$ & $1.82(0.39)$ \\
Green space & $1-2$ & $2.82(0.39)$ \\
General upkeep & $2-3$ & $1.24(0.44)$ \\
Social Environment* & & \\
PA activity initiator & $1-2$ & $1.88(0.86)$ \\
Policy-related Environment* & & $1.59(0.51)$ \\
Open before school hours & $1-4$ & $1-2$ \\
Ball games policy & & \\
* $=$ Only included variables in analyses are described; Non-included variables were excluded based on a lack of \\
variety between schools, e.g. all schools used scattered recess periods
\end{tabular}




\section{Schoolyard MVPA, SB, and average CPM}

In total, children had 47.3 minutes of MVPA a day (SD = $20.4 \mathrm{~min})$. Boys engaged in significantly higher levels of daily MVPA than girls (54.5 vs 41.0 minutes MVPA). The mean CPM score was 592 (SD = 209 CPM), with boys having higher CPM scores than girls. No gender difference was found for sedentary behavior. On average, children were sedentary for 8 hours and 13 minutes (SD $=1 \mathrm{~h} 21 \mathrm{~m}$ ) a day during waking hours.

Mean time spent at schoolyards was 11.3 minutes (SD $=3.3 \mathrm{~min}$ ) during morning recess, 25.5 minutes $(S D=15.3 \mathrm{~min}$ ) during afternoon recess, 14.7 minutes $(S D=9.8$ $\mathrm{min}$ ) outside school hours, and 54.1 minutes ( $\mathrm{SD}=22.4 \mathrm{~min}$ ) for total day schoolyard visits. The schoolyard contributed 8.7 minutes of daily MVPA (18.3\% of daily MVPA). Boys had more MVPA (10.5 minutes; $19.2 \%$ of daily MVPA) than girls (7.2 minutes; $17.6 \%$ of daily MVPA) at schoolyards. Similar values were found for CPM (1486 vs. 1164 CPM) at schoolyards. On average, children spent 20 minutes a day sedentary at the schoolyard (4.1\% of daily sedentary behavior; Table 6.2 ), with no difference being found between boys and girls.

Table 6.2: Schoolyard moderate-to-vigorous physical activity (MVPA) and sedentary behavior (SB) in minutes and counts-per-minute (CPM) by time period; total and stratified by gender

\begin{tabular}{|c|c|c|c|c|}
\hline Total day & Total $(\mathrm{N}=257)$ & Boys $(N=120)$ & Girls (N = 137) & Boys-Girls $p(t)$ \\
\hline MVPA & $47.29(20.37)$ & $54.47(20.82)$ & $40.99(17.78)$ & $<.01(5.596)$ \\
\hline SB & $493.30(82.13)$ & $484.73(87.57)$ & $500.81(76.60)$ & ns \\
\hline CPM & $592(209)$ & $641(204)$ & $548(204)$ & $<.01(3.632)$ \\
\hline Morning recess & Total $(\mathrm{N}=172)$ & Boys $(N=82)$ & Girls (N = 90) & Boys-Girls p (t) \\
\hline MVPA & $1.97(1.96)$ & $2.43(2.21)$ & $1.55(1.60)$ & $<.01(2.983)$ \\
\hline SB & $4.23(3.12)$ & $3.51(3.12)$ & $4.89(2.99)$ & $<.01(-2.956)$ \\
\hline CPM & 1395(1017) & 1655 (1113) & $1158(860)$ & $<.01(3.255)$ \\
\hline Afternoon recess & Total $(\mathrm{N}=167)$ & Boys $(N=76)$ & Girls (N = 91) & Boys-Girls $p(t)$ \\
\hline MVPA & $4.83(4.40)$ & $5.88(4.53)$ & $3.96(4.11)$ & $.01(2.878)$ \\
\hline SB & $8.95(7.28)$ & $8.67(7.11)$ & $9.18(7.45)$ & ns \\
\hline CPM & $1517(793)$ & $1708(744)$ & $1357(744)$ & $<.01(2.909)$ \\
\hline Outside school hours & Total $(N=153)$ & Boys $(N=69)$ & Girls $(N=84)$ & Boys-Girls p (t) \\
\hline MVPA & $1.58(1.88)$ & $1.83(2.18)$ & $1.38(1.58)$ & ns \\
\hline SB & $6.38(5.96)$ & $6.54(7.20)$ & $6.26(4.74)$ & ns \\
\hline CPM & $1006(483)$ & $1127(544)$ & $907(402)$ & .01 (2.799) \\
\hline Schoolyard total day & Total ( $N=117)$ & Boys $(N=52)$ & Girls (N = 65) & Boys-Girls p (t) \\
\hline MVPA & $8.67(6.34)$ & $10.48(6.59)$ & $7.22(5.79)$ & $.01(2.845)$ \\
\hline SB & $20.16(12.00)$ & $18.82(12.62)$ & $21.24(11.46)$ & ns \\
\hline CPM & 1304 (620) & $1486(615)$ & $1164(590)$ & $.01(2.871)$ \\
\hline
\end{tabular}

MVPA = moderate-to-vigorous physical activity; $\mathrm{SB}=$ sedentary behavior; $\mathrm{CPM}=$ counts per minute; duration is expressed in minutes 


\section{Correlates of schoolyard MVPA and SB by time period}

Multilevel analyses were conducted to study correlates of children's MVPA and SB for total day schoolyard visits (Table 6.3) and for each time period at the schoolyard (Table 6.4). Only the final model (i.e. associations with $p<.10$ between physical, social, and policy-related schoolyard characteristics and MVPA and SB) is presented. The periodspecific analyses showed that male gender was a positive correlate of MVPA.

As regards total day schoolyard visits $(N=117)$, the presence of high bars was positively associated with MVPA. The presence of small soccer goals and the presence of high bars were negative associated with SB. Furthermore, the absence (or limited presence) of green space at schoolyards was associated with less SB. A positive association was found between the available square meters per child and SB, while the presence of a ball games area policy was negatively associated with SB (Table 6.3).

Table 6.3: Schoolyard correlates of MVPA and SB during total day schoolyard visits ( $N=117)$

\begin{tabular}{|c|c|c|c|c|}
\hline \multirow[t]{2}{*}{ Total day schoolyard visits $(\mathrm{N}=117)$} & \multicolumn{2}{|l|}{ MVPA } & \multicolumn{2}{|l|}{ SB } \\
\hline & Beta (95\%C.I.) & $p$ & Beta (95\%C.I.) & $p$ \\
\hline \multicolumn{5}{|l|}{ Demographics } \\
\hline Gender (male) & $3.50(1.34-5.67)$ & $<.01$ & & ns \\
\hline \multicolumn{5}{|l|}{ Physical Environment } \\
\hline Small soccer goals & & ns & $-6.89(-12.81--2.67)$ & $<.01$ \\
\hline High bars & $0.49(0.13-0.85)$ & .01 & $-0.77(-1.37--0.17)$ & .01 \\
\hline Green space (limited) & & ns & $-12.52(-20.23--4.81)$ & $<.01$ \\
\hline Square meters per child & & ns & $0.80(0.02-1.62)$ & $<.01$ \\
\hline \multicolumn{5}{|l|}{ Policy Environment } \\
\hline Ball games area policy & & ns & $-10.53(-16.64--4.41)$ & $<.01$ \\
\hline
\end{tabular}

MVPA = moderate-to-vigorous physical activity; SB = sedentary behavior

As regards morning recess ( $N=172)$, the presence of high bars was associated with more MVPA. Another positive correlate of MVPA was PA activities initiated by teachers rather than initiated by children only. The presence of high bars and teacher-initiated PA activities were also associated with less SB (Table 6.4).

As regards afternoon recess $(N=167)$, the presence of high bars was associated with higher levels of MVPA. Available square meters per child was positively associated with both MVPA and SB. Children in grade 6 were less involved in SB at schoolyards than children in grade 7 (Table 6.4).

As regards outside school hours $(N=153)$, opening hours of the schoolyard before the school day begins were positively associated with MVPA. None of the other schoolyard characteristics were significantly $(p<.05)$ associated with SB at schoolyards outside school hours (Table 6.4). 
Table 6.4: Schoolyard correlates of MVPA and SB by time periods; morning recess $(\mathrm{N}=172)$, afternoon recess $(\mathrm{N}=167)$, and outside school hours ( $\mathrm{N}=153)$

\begin{tabular}{|c|c|c|c|c|}
\hline \multirow[t]{2}{*}{ Morning recess $(\mathrm{N}=172)$} & \multicolumn{2}{|l|}{ MVPA } & \multicolumn{2}{|l|}{ SB } \\
\hline & Beta (95\%C.I.) & $p$ & Beta (95\%C.І.) & $p$ \\
\hline \multicolumn{5}{|l|}{ Demographics } \\
\hline Gender (male) & $0.89(0.33-1.45)$ & $<.01$ & $-1.39(-2.24--0.53)$ & $<.01$ \\
\hline \multicolumn{5}{|l|}{ Physical Environment } \\
\hline Small soccer goals & $0.59(-0.11-1.28)$ & .09 & & ns \\
\hline High bars & $0.12(0.03-0.20)$ & .01 & $-0.26(-0.38--0.15)$ & $<.01$ \\
\hline Markings & & ns & $0.07(-.01-0.15)$ & .07 \\
\hline \multicolumn{5}{|l|}{ Social Environment } \\
\hline PA initiator (child only) & $-0.87(-1.64--0.11)$ & .03 & $1.26(0.32-2.20)$ & .01 \\
\hline \multirow[t]{2}{*}{ Afternoon recess $(\mathrm{N}=167)$} & \multicolumn{2}{|l|}{ MVPA } & \multicolumn{2}{|l|}{ SB } \\
\hline & Beta (95\%C.I.) & $p$ & Beta (95\%C.I.) & $p$ \\
\hline \multicolumn{5}{|l|}{ Demographics } \\
\hline Gender (male) & $2.07(0.86-3.27)$ & $<.01$ & & ns \\
\hline Grade (6) & & ns & $-4.37(-6.36--2.39)$ & $<.01$ \\
\hline \multicolumn{5}{|l|}{ Physical Environment } \\
\hline High bars & $0.40(0.24-0.56)$ & $<.01$ & & ns \\
\hline General upkeep (limited) & & ns & $-3.66(-7.77-0.44)$ & .08 \\
\hline Square meters per child & $0.20(0.07-0.33)$ & $<.01$ & $0.48(0.14-0.83)$ & .01 \\
\hline \multirow[t]{2}{*}{ Outside school hours ( $N=153$ ) } & \multicolumn{2}{|l|}{ MVPA } & \multicolumn{2}{|l|}{ SB } \\
\hline & Beta (95\%C.I.) & $p$ & Beta (95\%C.I.) & $p$ \\
\hline \multicolumn{5}{|l|}{ Physical Environment } \\
\hline General upkeep (limited) & & ns & $3.14(-0.59-6.87)$ & .09 \\
\hline \multicolumn{5}{|l|}{ Policy Environment } \\
\hline Open before school hours & $0.58(0.15-1.01)$ & .01 & & ns \\
\hline Ball games area policy & $0.67(-0.05-1.40)$ & .07 & & ns \\
\hline
\end{tabular}

MVPA = moderate-to-vigorous physical activity; SB = sedentary behavior

\section{DISCUSSION}

The aim of this study was to identify the correlates of MVPA and SB in the schoolyard environment of children aged 8-11 years, using accelerometry and GPS data. On average, children spent almost 9 minutes (18\%) of their MVPA at schoolyards, and 20 minutes (37\%) of their SB. The time spent in MVPA at the schoolyard is comparable with the findings of previous research combining accelerometer data and GPS data (Dessing et al., 2013; Klinker et al., 2014a; Klinker et al., 2014b). The percentage of time spent in SB at schoolyards was slightly lower than in other studies (Ridgers et al., 2010b; Willenberg et al., 2010). Boys engaged in more MVPA at schoolyards than girls (except outside school hours) which was in line with most previous research (Ridgers et al., 2012a). During the morning recess, boys showed lower levels of SB than girls (Ridgers et al., 2010b). We tried to identify schoolyard characteristics that were associated with MVPA and SB in children. 
As regards the physical schoolyard environment, the presence of high bars and soccer goals were the most consistent correlates of more MVPA and less SB. Several other studies found that fixed equipment on schoolyards were positively associated with PA, but they were less specific, in that physical features were merely defined as 'fixed equipment' rather than specified by type of equipment (Nielsen, Taylor, Williams, \& Mann, 2010; Ridgers et al., 2010b; Willenberg et al., 2010). Our findings indicate the need to differentiate between types of fixed equipment. Specifically, this differentiation showed that in our study playground markings were not positively associated with children's PA. This is in contrast with other studies, which found positive associations between playground markings and MVPA (Stratton \& Mullan, 2005; Willenberg et al., 2010). However, a recent review by Broekhuizen reported a lack of association between playground markings and PA in other studies (Broekhuizen et al., 2014). These inconsistencies could partly be explained by the type of marking, as these can differ substantially, and our results may have been affected by insufficient ageappropriateness of the playground markings. Most of the markings in our schoolyards were generally intended to be used by young children (see example in Figure 6.1), whereas our study focused on older primary school children. An increased number of available square meters per child was associated with both more MVPA and more SB. These findings are in line with Ridgers et al. (2010b), who found the same pattern. There may be a kind of optimum for available space per child. Another explanation for this pattern could be that more available space per child enhances MVPA, which leads to compensation behavior (SB) in the same environment. This explanation follows the tenets of the 'activitystat' hypothesis assuming that an increase or decrease in activity will lead to compensation during the rest of the day (Rowland, 1998). In the current study, the compensation occurs immediately, i.e. while being in the same recess period. This compensation behavior was only found during the afternoon recess and not during other time periods, which in accordance to previous studies does not provide a clear indication to support or reject the 'activitystat' hypothesis (Baggett et al., 2010; Gomersall, Rowlands, English, Maher, \& Olds, 2013). Moreover, as the association between square meters and MVPA and SB was only found in the afternoon recess, it could also be a result of lunchtime as concurrent sedentary schoolyard activity. In contrast to previous research (Dyment, Bell, \& Lucas, 2009), we found that the presence of green space at schoolyards enhanced SB, whereas we found no association with MVPA. The most likely explanation for this finding is the design of the schoolyard green spaces examined in the current study, which mostly had an aesthetic function, e.g. trees and hedges, rather than providing a green surface that could encourage PA, such as grass (soccer) fields. 


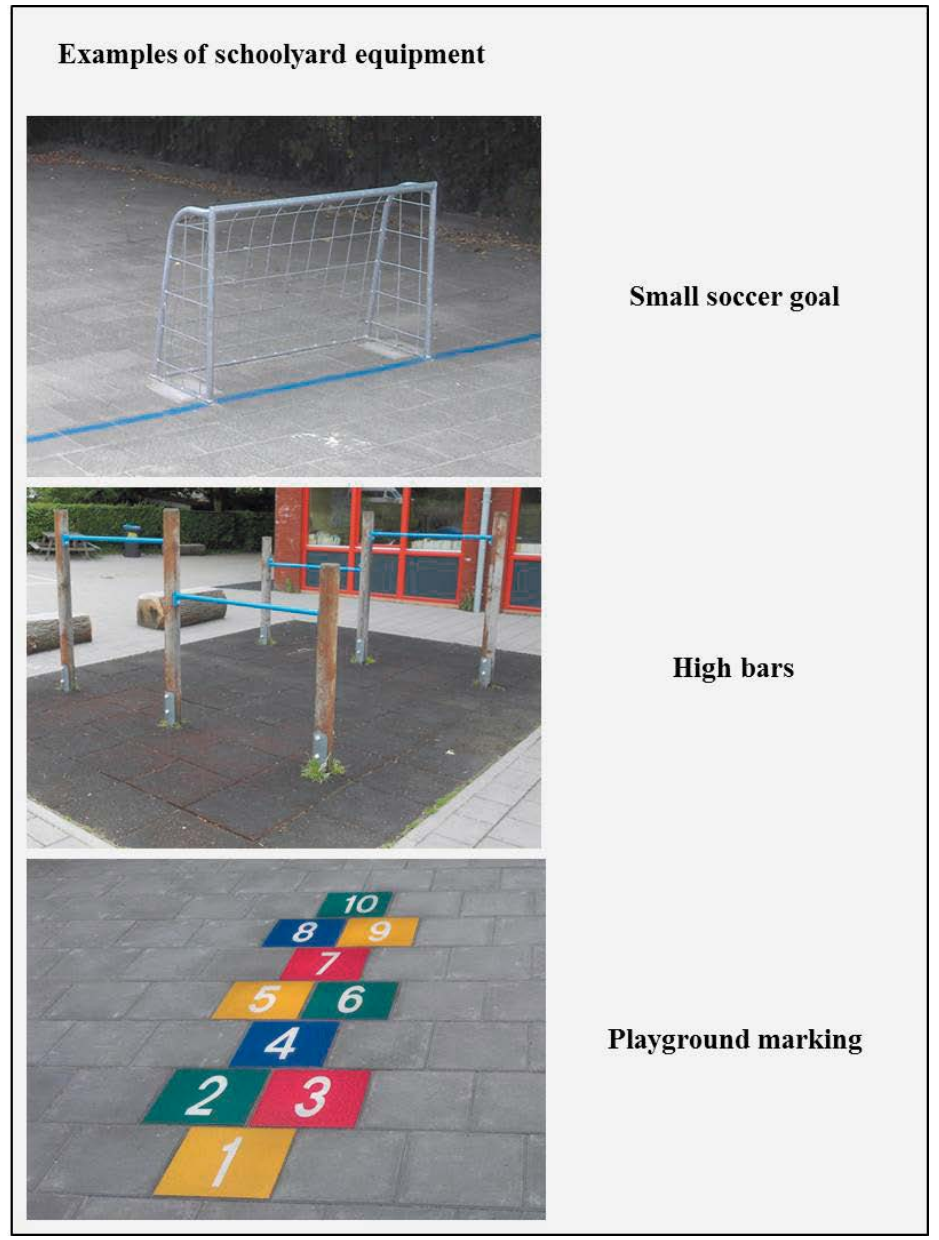

Figure 6.1: Examples of assessed schoolyard play equipment

There was also an association between the social and policy environments and MVPA and SB. Teacher involvement in the initiation of PA activities was associated with higher levels of MVPA and less SB. Teachers are role models for children in this age group, and might enhance MVPA or prevent SB during recess (Brown et al., 2009; Gubbels et al., 2011; Huberty et al., 2011). Teachers' awareness of their function during recess is therefore essential in order to encourage PA. Teachers' predominant position in activating children during recess might be (partly) taken by other adult role models, such as parents or (municipal) sports coaches (Janssen et al., 2015). This could potentially prevent teachers' perception of task overloads, but underlines the enthusing and encouraging role of adult supervisors to promote PA and prevent SB during recess. The presence of a ball games area policy was a correlate of lower SB during morning recess. Setting and enforcing rules that indicate where children are allowed to play with 
balls may encourage the use of certain ball play areas, hence decreasing the opportunity to use such areas for SB. At the same time, it creates a safer and more open situation for playing in areas where ball games are banned. Finally, opening hours of schoolyards were positively associated with levels of MVPA outside school hours. In line with a shared use model, this finding argues for extending opening hours of schoolyards to intensify their use (Kanters et al., 2014).

\section{Strengths and limitations}

A major strength of this study is the use of both objectively measured PA levels (accelerometer) and location (GPS) data, combined with physical, social, and policyrelated schoolyard characteristics. This approach enabled us to correlate children's PA behavior with its behavioral context more accurately than has been done in ecological studies focusing on the influences of less specified physical environments on PA behavior (Jankowska et al., 2015). For example, in some schools we found that children did not take recess at the time they were supposed to do so according to their schedule. Without the use of GPS devices, non-users of the schoolyard environment would have been included in our analyses. Our novel data processing technique enabled us to exclude these non-schoolyard data, leading to more accurate associations between environmental features and PA behavior. Moreover, to improve (international) comparability between studies we applied a novel data processing technique that was recently tested successfully in processing children's PA levels and location data simultaneously (Klinker et al., 2015). In spite of this, more studies using GPS and accelerometer data are needed in the schoolyard environment to improve the generalizability of our results.

This study also had limitations. The variation in schoolyard characteristics was limited, affecting the statistical power of our analyses. In addition, sample size within each school was relatively small. A minimum of 5 children was required within the schools to be included in the study, leading to insufficient power in the statistical analyses. Moreover, loss of data $(N=3)$ occurred due to technical failures in one or both devices or due to insufficient memory space on the GPS devices. Some children forgot to charge the battery overnight, resulting in fewer valid days for inclusion in the analyses. This means that future studies using these devices should considerably oversample (Klinker et al., 2014b). We recommend using a variety of schools and a sufficiently large sample size within each school in future studies. Another limitation was that the schools' schoolyard policies were assessed by telephone interviews with the school principals, which could have led to some socially desirable answers. At last, since our study had a cross-sectional design, it was only able to identify schoolyard correlates for MVPA and SB, rather than causal relationships. 


\section{Conclusion}

The schoolyard environment offers a window of opportunity to increase the PA levels of primary school children and prevent sedentary behavior. Schoolyards account for almost one fifth of total day MVPA for both boys and girls. This study identified specific schoolyard characteristics that were associated with more MVPA, and found that the presence of fixed equipment, such as high bars and soccer goals, was most consistently positively associated with MVPA and negatively with SB. Furthermore, the study showed the importance of the social and policy-related environment at schoolyards, indicating the need for interventions that include these types of environment in addition to the physical environment.

\section{IMPLICATIONS FOR SCHOOL HEALTH}

From a health practitioner's or school principal's perspective, this study provides some indications of the kind of environmental variables that can contribute to increased levels of MVPA or decreased SB among children. In addition to environmental influences, special attention needs to be paid to girls' PA in general, since girls engaged in significantly less MVPA than boys. Based on this study, we recommend the presence of fixed equipment, such as high bars and soccer goals at schoolyards, to increase children's MVPA levels and prevent SB. When designing or redesigning a schoolyard, one could critically consider what amount and type of green space could be incorporated in the design and what its function should be. We also recommend that schools establish rules indicating certain areas where ball games are allowed, rather than allowing children to play ball games everywhere. In addition, it would be advisable to create awareness among teachers that they function as role models for children and that they have opportunities to enhance MVPA and prevent SB by initiating activities at schoolyards. If teachers' heavy workload should make this difficult to realize, another option would be to use other role models (e.g. parents) at schoolyards to initiate PA activities. Finally, expanding the opening hours of schoolyards could contribute to more MVPA. 


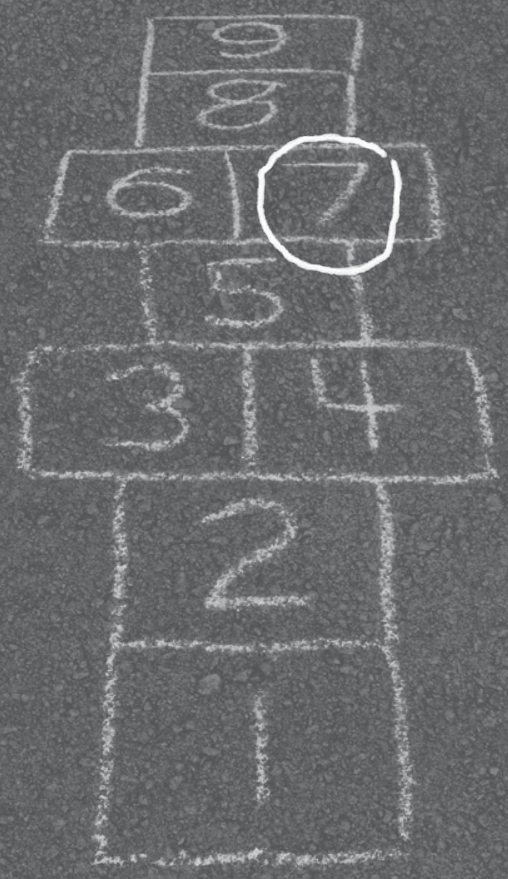

\section{Chapter 7}

\section{A multicomponent schoolyard intervention targeting children's recess physical activity and sedentary behavior: Effects after one year}

This chapter is based on: Van Kann, D. H. H., de Vries, S. I., Schipperijn, J., de Vries, N. K., Jansen M. W. J. \&.Kremers, S.P. J. A multicomponent schoolyard intervention targeting children's recess physical activity and sedentary behavior: Effects after one year. Submitted for publication. 


\section{ABSTRACT}

Background Aim of the study was to test the 12-month effects of a multicomponent physical activity (PA) intervention at schoolyards on recess PA levels of $6^{\text {th }}$ and $7^{\text {th }}$ grade children in primary schools, combining Global Positioning System (GPS) and accelerometry data.

Methods A quasi-experimental study design was used with 20 paired intervention and control schools. GPS confirmatory analyses were applied to validate attendance at schoolyards during recess. Data from 376 children from 7 pairs of schools were included in the final analyses. Pooled intervention effectiveness was tested by multilevel linear regression analyses, whereas effectiveness of intervention components was tested by multivariate linear regression analyses.

Results Children exposed to the multicomponent intervention increased their time spent in light PA (+5.9\%) during recess. No pooled effects on Moderate-to-Vigorous PA were found. In depth-analyses on intervention components showed that physical schoolyard interventions particularly predicted a decrease in time spent in sedentary behavior during recess at follow-up. Intervention intensity and school's commitment to the project strengthened this effect.

Conclusions The multicomponent schoolyard PA intervention was effective in making children spend a larger proportion of recess time in light PA, which was most likely the result of a shift from sedentary behavior to light PA. 


\section{BACKGROUND}

Physical activity (PA) is positively associated with a range of health, psychosocial, and academic outcomes in youth (Andersen et al., 2011; Biddle \& Asare, 2011; Loprinzi et al., 2012; Rothon et al., 2010; Trudeau \& Shephard, 2010). Hence, the current decreasing PA levels and increasing levels of sedentary behavior (SB) in children are worrying, especially since activity patterns and sedentary patterns in childhood seem to track into adulthood (Singh et al., 2008). Intervening at an early age might therefore be essential and has great potential in terms of beneficial outcomes at population level in later life. The sustained effect of current PA interventions on children's daily PA levels, however, is small (Metcalf, Henley, \& Wilkin, 2012; Sims, Scarborough, \& Foster, 2015), which emphasizes the need to better understand how PA interventions should be developed in order to become more effective.

Schools are suitable environments to reach children and create substantial impact with interventions (Van Sluijs, McMinn, \& Griffin, 2007). Schoolyards can influence children's PA and SB, especially during recess periods. In recent years, several reviews have been published about the association between physical and/or social attributes of the schoolyard environment on PA and the effect of physical and social schoolyard interventions on PA (Broekhuizen et al., 2014; Escalante et al., 2014; Parrish et al., 2013; Ridgers et al., 2012a; Stanley et al., 2012). These reviews provide useful indications as to what attributes potentially contribute to more PA and less SB at schoolyards. Although often studied in cross-sectional research designs or short-term effect studies, the availability of fixed and portable equipment has most consistently and positively been associated with higher PA levels (Colabianchi, Maslow, \& Swayampakala, 2011; Ridgers et al., 2010b; Willenberg et al., 2010). Studies regarding long-term effects, i.e. $\geq 12$ months, of schoolyard interventions on children's PA levels using controlled study designs are scarce (Ridgers, Fairclough, \& Stratton, 2010a).

Most schoolyard interventions at primary schools were found to be delivered as multicomponent interventions rather than as isolated attributes (Broekhuizen et al., 2014; Parrish et al., 2013). These ecological systems approaches seem to have greater impact than isolated physical or social environment interventions (Huberty et al., 2011; Loucaides, Jago, \& Charalambous, 2009; Ridgers et al., 2010a; Ridgers, Stratton, Fairclough, \& Twisk, 2007). A core feature of ecological systems theory is the interaction of elements of the environment in shaping human behavior (Kremers et al., 2006; Sallis et al., 2006), and testing moderators of environmental influences is the most commonly mentioned suggestion for future research (Gubbels et al., 2014).

Although SB and PA have been identified as distinct constructs and independent risk factors for several health risks in children (Pearson et al., 2014; Saunders et al., 2014) environmental interventions at schoolyards aimed at reducing SB have been scarce (Blaes et al., 2013; D'Haese, Van Dyck, De Bourdeaudhuij, \& Cardon, 2013). Only one experimental schoolyard study has been designed to reduce sedentary time. This study 
showed that increasing the number of square meters of schoolyard per child (by reducing the number of children present at the schoolyard at once) contributed to less SB (D'Haese et al., 2013). Ongoing schoolyard intervention studies (Andersen et al., 2015), however, tend to focus more on limiting SB in addition to encouraging PA.

The use of GPS data in addition to accelerometer data has been advocated in health behavior studies in order to enrich data with location-specific information (Jankowska et al., 2015), allowing researchers to more accurately specify the use of physical environments in relation to PA and SB. The combination of GPS data and accelerometry at schoolyards has previously been used in only a few studies (Dessing et al., 2013; Klinker et al., 2014a; Klinker et al., 2014b), all using a cross-sectional study design. To date, no longitudinal (schoolyard) intervention studies have used this approach.

The primary goal of the project under study was to determine sustained changes in children's PA levels due to environmental changes. The focus of the environmental changes was on enhancing sustainable PA changes in primary school children after one year of implementation, rather than studying immediate intervention effects on children's PA. Therefore, the aim of the current study was to examine the 12-month effect of multicomponent schoolyard interventions on 8-to-11 years old primary school children's physical activity levels during recess, in terms of sedentary behavior (SB), light physical activity (LPA), and moderate-to-vigorous physical activity (MVPA), using a combination of accelerometry and GPS data. Moreover, we tested the moderating effect of the intensity of the schoolyard intervention and the commitment of schools to the intervention on the relation between physical and social schoolyard interventions and children's PA levels to better understand contextual factors that might influence the long-term effect of schoolyard interventions on PA levels.

\section{METHODS}

\section{Recruitment of schools and participants}

Data used in this study were derived from the Active Living project (ZonMw Project Number 200130003; (Van Kann et al., 2015a)). The Active Living project started in 2012 and is a 3-year project focusing on increasing PA among children by means of adjustments to the physical and social environment of primary schools situated in deprived areas. The Active Living project used a quasi-experimental study design in which 10 schools served as intervention schools and 10 schools as controls. Control schools were matched to intervention schools on geographical characteristics, including level of neighborhood deprivation and urbanization. In addition, control schools were not located within an $800 \mathrm{~m}$ radius of an intervention school. All schools were situated in deprived neighborhoods in the Southern Limburg area in the Netherlands. Moreover, included schools executed a 'regular' Dutch school system, i.e. no schools for children 
with special needs were included. Children in grades 6 and 7 (between 8-11 years old at baseline) were invited to participate in this project. This age range was selected because children at this age become able to travel and move around independently (Panter et al., 2008; Timperio et al., 2004). Baseline data for the current study were gathered between April and June 2013, prior to any interventions being conducted at schools. The follow-up measurement took place after physical and social interventions in the schoolyard environment had been implemented between April and June 2014. As part of the Active Living project children were asked to fill out a questionnaire ( $N=1340$ at baseline; $\mathrm{N}=1322$ at follow-up), including demographic information. In addition, they were asked to wear an accelerometer, the ActiGraph GT3X+ (N=791; 59\% at baseline; $\mathrm{N}=740 ; 56 \%$ at follow-up), and a random selection of them (due to limited availability of devices) were asked to wear a GPS device, the QStarz BT1000-XT. Before they started wearing the device (or both devices), written consent was obtained from their parents and oral consent from the children. The children nor their parents did receive an incentive for participation in the study. This study was given ethical approval by the ethics committee of the Maastricht University Medical Centre (reference number METC 12-4-077).

\section{Interventions}

Schools were supported in implementing physical schoolyard interventions (PSIs) and social schoolyard interventions (SSIs) to stimulate children's PA, e.g. providing new fixed equipment and/or teachers introducing schoolyard games. Schools took part in working groups, chaired by a municipal health service employee, to identify the schools' needs for environmental changes. Interventions were tailored to the schools' needs resulting in a variety of implemented interventions. Each school received a small working budget $(2,000$ euros per school) to initiate interventions. If the planned interventions exceeded the budget, this had to be covered by sponsoring or external investment. Non-drastic schoolyard interventions with a limited magnitude and interventions not exceeding the budget were considered to be of low intensity, whereas drastic schoolyard interventions with a large magnitude and interventions substantially exceeding the budget were categorized as interventions with a high intensity. The number of different types of PSIs and SSIs, i.e. different environmental stimuli, and the intensity of PSIs were monitored by the municipal health service employees, who kept diaries of activities performed. The municipal health service employees also kept a diary on the schools' project commitment by scoring the level of attention given to the project in a school and external manifestations of the project, such as communications in parental newsletters and the presence of posters in school, on a 4-point scale (1=low-4=high; Table 7.1). 
Table 7.1: Overview of physical (PSIs) and social schoolyard interventions (SSIs) at each school, the intensity of PSIs and the schools' level of commitment in the project

\begin{tabular}{|c|c|c|c|c|c|c|c|}
\hline Physical schoolyard intervention (PSI) & $\mathrm{I}_{1}$ & $\mathrm{I}_{2}$ & $I_{3}$ & $\mathrm{I}_{4}$ & $I_{5}$ & $I_{6}$ & $\mathrm{I}_{7}$ \\
\hline New fixed equipment in schoolyard & + & + & + & + & - & + & + \\
\hline New loose equipment in schoolyard & + & - & + & + & + & - & + \\
\hline Playground markings & + & - & - & + & + & - & - \\
\hline Dedicated ball games area & + & - & + & + & - & + & - \\
\hline Sound equipment in schoolyard environment & + & - & - & - & - & - & - \\
\hline Number of PSIs & 5 & 1 & 3 & 4 & 2 & 2 & 2 \\
\hline Intensity (1=low; 2=high) & 1 & 1 & 2 & 1 & 1 & 2 & 1 \\
\hline \multicolumn{8}{|l|}{ Social schoolyard intervention (SSI) } \\
\hline Additional sports day in schoolyard & + & + & - & + & + & + & - \\
\hline Sports clinics during recess & + & + & + & + & + & - & + \\
\hline Use of schoolyard games & + & - & - & - & + & + & - \\
\hline Number of SSIs & 3 & 2 & 1 & 2 & 3 & 2 & 1 \\
\hline Project commitment (1=low-4=high) & 4 & 3 & 3 & 2 & 2 & 2 & 3 \\
\hline
\end{tabular}

I = intervention school; + = implemented; - = not implemented

\section{Measures}

\section{Activity measures}

Children were asked to wear an accelerometer (ActiGraph GT3X+; 30Hz, $10 \mathrm{sec}$ epoch) during waking hours for at least 5 consecutive days, including a weekend (i.e. three weekdays and two weekend days). Children's PA data were analyzed using ActiLife version 6.10.4 (ActiGraph, USA), while Evenson's cut-off values (Evenson et al., 2008) were used to classify SB, LPA, and MVPA. We used a 480 minutes (8 hours) daily wear time as validation check for inclusion in the analyses (Jago et al., 2013) to prevent the inclusion of children that only wore their devices while in recess, which was considered as a proxy for a measurement effect, i.e. children wearing the device only during recess were assumed to be very aware of wearing the device which probably increases the risk of overestimating PA during recess. Morning recess data were extracted from class time tables for each child. Weekend days were excluded, as was the first day of wearing the devices, due to potential reactivity to the measuring equipment (Dössegger et al., 2014), while the remaining wearing days were checked for validity. Children spending over $90 \%$ of the recess epochs in SB were excluded, since it was considered unlikely that these children wore their device appropriately while in recess based on a feasibility check on walking time between the classroom and the schoolyard. Valid recess data (with a minimum of 1 school day) were aggregated per child for the morning recess period. We only included the morning recess period as this was the only recess period in which all children stayed at school (i.e. lunch breaks at school were not mandatory at all participating schools). To correct for different recess durations across schools, the 
proportion of time spent in SB, LPA and MVPA was the main outcome measure. Change scores between baseline and follow-up were calculated by subtracting the percentage of time spent at a particular activity level at baseline from the percentage of time spent at that activity level at follow-up.

\section{Confirmatory analyses based on GPS data}

To examine whether children were actually present at schoolyards during recess, we compared GPS data on schoolyard presence with class time tables. In total, 120 children wore a GPS device at baseline and 537 at follow-up. PALMS (the Personal Activity and Location Measurement System; UCSD, USA) was used to merge accelerometer and GPS data. A purpose-built PostgreSQL database was used to combine the location-activity matched data with class time tables and Geographic Information System (GIS) data on schoolyards (an example is shown in Figure 7.1). Schools operated according an allindoors or all-outdoors policy during recess. If at least some of the children who wore a GPS were present at the schoolyard during recess, we assumed that all children in that class had the opportunity to use the schoolyard during the recess time listed in the class time table.
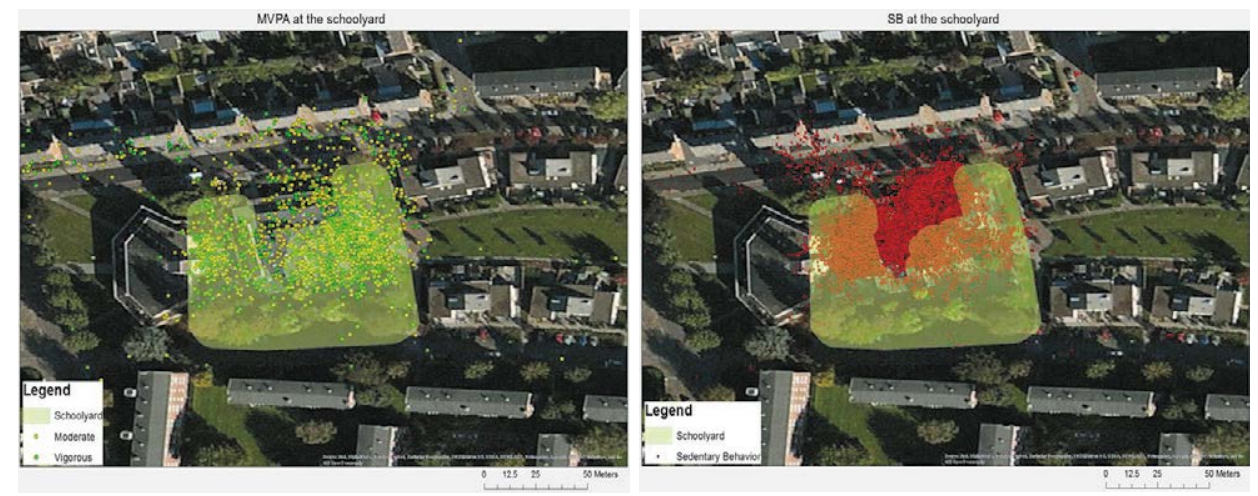

Figure 7.1: Example of defining PA levels in a schoolyard combining GPS and accelerometry (left-hand panel = MVPA; right-hand panel = SB; green area = schoolyard).

\section{Weather conditions}

Weather conditions (temperature, sun exposure, and precipitation) for every hour of a measurement day were obtained from the Royal Netherlands Meteorological Institute (KNMI). A change score was calculated for temperature, sun exposure, and precipitation between follow-up and baseline for each day of measurement. Weather data were aggregated in line with the aggregation of valid recess PA data. 


\section{Data analysis}

Data analyses were conducted using SPSS 20.0 (IBM Corp, USA) and a p-value of .05 was used as the significance level for both main effects and interaction effects (Fairchild \& Mackinnon, 2009). Firstly, we studied the pooled intervention effects of multicomponent interventions by means of multilevel regression analyses, using change scores of PA levels as the dependent variable. Gender, grade, ethnicity of the child, change in temperature, change in precipitation, change in hours of sunshine, intervention (experimental/control condition), and baseline PA levels (SB, LPA, and MVPA respectively) were entered as independent variables at level 1 , whereas school was entered as an independent variable at level 2. Other confounding factors, such as available square meters at the schoolyard and teacher supervision (Broekhuizen et al., 2014) were not controlled for as they stayed the same over time.

After defining the pooled effectiveness of the multicomponent schoolyard intervention, the effectiveness of different types of environmental interventions, intervention intensity and school's commitment to the Active Living project on PA change scores in the intervention schools were tested. Within the subsample of intervention schools three multivariate linear regression analyses were performed, with the change in time spent in SB, LPA, and MVPA as the dependent variable, and gender, grade, ethnicity of the child, change in weather conditions, number of PSIs, number of SSIs, level of commitment to the project, intervention intensity, and baseline PA levels as independent variables. To correct for seasonal effects and varying number of children in intervention and control schools, mean PA change scores for the matched control school were subtracted from individual PA change scores for the intervention school. Potential contextual factors affecting the influence of physical environmental interventions were tested by including interaction terms of the number of PSIs and the intensity and number of PSIs and project commitment in the analyses. In case of significant interaction, stratified analyses were performed.

\section{RESULTS}

\section{Description of population and time spent in PA and SB at baseline and follow-up}

In total, 740 children were asked to wear an accelerometer at follow-up of which 621 (84\%) provided valid PA data. These 621 children were checked for a valid PA measurement at baseline. Moreover, the confirmatory GPS analyses showed that data from 3 out of 10 pairs of schools (intervention-control) were invalid for the purpose of the current study, as no children used the schoolyard during recess at baseline and/or follow-up. These pairs of schools were removed from the study sample, resulting in the inclusion of 376 children from 7 pairs of schools (mean N per school=27) providing valid 
accelerometer data at both baseline and follow-up. Both at baseline and follow-up the number of children providing 1 valid day of PA data was 125 (33\%). The number of children enrolled in the intervention schools (7 schools; 215 children) was slightly higher than in the control schools (7 schools; 161 children). There was a slight overrepresentation of children in grade 6 (55\%) compared to children in grade 7 (45\%). Two hundred girls (53\%) were included and 176 boys (47\%). The average size of the included schoolyards was 1984 square meters (approximately 15 square meters per child) and all schools provided fixed and loose equipment to their children at baseline.

On average, schools implemented 2.7 PSIs, whereas $2.0 \mathrm{SSIs}$ per school were implemented. Two schools implemented high-intensity PSIs, and 4 out of 7 schools scored (very) high on project commitment (Table 7.1). Recess time lasted on average 15.3 minutes ( $S D \pm 1.4$ ) and did not significantly change over time. At baseline, children spent an average of $43 \%$ (SD \pm 20$)$ of recess time in SB, $42 \%(S D \pm 12)$ in LPA, and $15 \%$ (SD \pm 11 ) in MVPA. At follow-up, the average time spent in SB and LPA was $42 \%$ (SD \pm 21 and SD \pm 13 respectively) and time spent in MVPA was $16 \%$ (SD \pm 15 ). Children in the intervention condition spent slightly less time in SB at baseline $(p=.06, t=1.88)$ and spent more time in MVPA at baseline than children in the control condition $(p<.01 ; t=-3.74)$.

\section{The pooled intervention effect on change in SB, LPA, and MVPA after 12 months}

In the intervention schools, a 5.9\% decrease in SB and a 5.4\% increase in LPA were found after correcting for effects in the matched control schools (Figure 7.2). The decrease in the time spent in SB was not significant, but the increase in the time spent in LPA was. No difference was found between control and intervention schools regarding change in MVPA (Table 7.2). Female gender was a significant predictor of more SB during the follow-up measurement, as well as a significant predictor of decreased proportion of time spent in LPA and MVPA after 12 months. Children attending grade 7 at baseline spent more time in SB at follow-up. Furthermore, decreased time spent in SB after 12 months was predicted by more sun exposure during the follow-up measurement. Additionally, more sun exposure during the follow-up measurement predicted an increase in LPA after 12 months. As regards MVPA, children attending grade 7 at baseline were less likely to show increased time spent in MVPA at follow-up.

\section{What intervention components predict change in SB, LPA and MVPA over time?}

To study the effectiveness of implemented intervention components, additional indepth linear regression analyses were conducted among intervention schools only. Change in the time spent in SB was affected by the number of PSIs that was implemented at schoolyards. More PSIs resulted in less SB at schoolyards after 12 months. The number of PSIs also showed a trend towards predicting a higher 
proportion of recess time spent in LPA and MVPA ( $p<.10)$. In addition, implementation of interventions with a high intensity led to a larger decrease in the time spent in SB and a larger proportion of time spent in LPA and MVPA at 12-month follow-up. Neither the number of SSIs nor the level of project commitment had a main effect on the changes in SB, LPA, and MVPA (Table 7.3). We found a significant interaction effect between the number and intensity of PSIS (SB $p<.01$; LPA $p=.03$, MVPA $p<.01$ ). Stratified analyses showed that the decrease in SB, and increases in LPA and MVPA, were largest for children attending schools that implemented more and higher intensity PSIs. As regards change in the time spent in LPA and MVPA, an interaction effect was found for PSIs and project commitment (LPA $p=.03$; MVPA $p=.05$ ). PSIs implemented in highly committed schools showed better results. As regards MVPA, stratified analyses (low $(\leq 2)$ versus high $(\geq 3)$ commitment) showed a positive though non-significant effect of the number of PSIs and high project commitment on MVPA at follow-up (std. $\beta=.241 ; p=.16$ ), but an increased number of PSIs and low project commitment showed a negative trend in terms of MVPA at follow-up (std. $\beta=-.250 ; p=.08$ ).

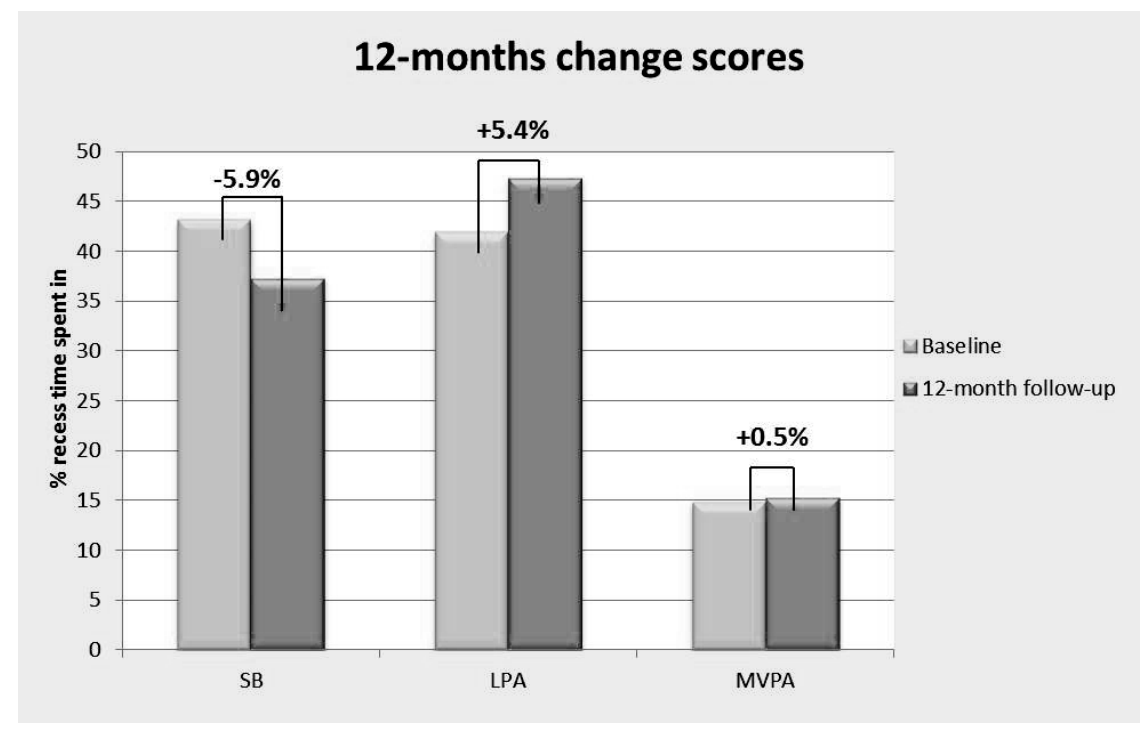

Figure 7.2: 12-month follow-up change scores for SB, LPA and MVPA in which the effect in intervention schools is adjusted for that in control schools. 
Table 7.2: Predictors of change scores in percentage of recess time spent in SB, LPA, and MVPA

\begin{tabular}{|c|c|c|c|c|c|c|}
\hline \multirow[t]{2}{*}{$(N=376)$} & \multicolumn{2}{|l|}{ SB } & \multicolumn{2}{|l|}{ LPA } & \multicolumn{2}{|l|}{ MVPA } \\
\hline & Beta (95\%C.I.) & $p$ & Beta (95\%C.I.) & $p$ & Beta (95\%C.I.) & $p$ \\
\hline Baseline \% in PA level & $-0.88(-0.99--0.77)$ & $<.01$ & $-0.88(-0.98--0.79)$ & $<.01$ & $-0.76(-0.87--0.65)$ & $<.01$ \\
\hline Grade (6) & $-3.96(-7.52--0.39)$ & .03 & $0.11(-2.23-2.45)$ & .93 & $3.20(0.49-5.90)$ & .02 \\
\hline Gender (male) & $-13.17(-16.72--9.61)$ & $<.01$ & $5.02(2.73-7.30)$ & $<.01$ & $6.46(3.70-9.23)$ & $<.01$ \\
\hline Ethnicity (Dutch) & $1.69(-7.58-10.96)$ & .72 & $-3.03(-9.11-3.03)$ & .33 & $1.40(-5.61-8.42)$ & .69 \\
\hline$\Delta$ in temperature $(\stackrel{\circ}{\circ})$ & $0.21(0.14-0.27)$ & $<.01$ & $-0.12(-0.15--0.09)$ & $<.01$ & $-0.11(-0.16--0.07)$ & $<.01$ \\
\hline$\Delta$ in sun exposure & $-1.35(-2.11--0.59)$ & $<.01$ & $1.10(0.78-1.42)$ & $<.01$ & $0.18(-0.38-0.74)$ & .53 \\
\hline$\Delta$ in precipitation & $1.40(-1.26-4.05)$ & .30 & $-0.02(-1.22-1.17)$ & .97 & $-0.59(-2.54-1.36)$ & .55 \\
\hline Intervention (exposed) & $-2.30(-10.48-5.88)$ & .54 & $2.40(0.09-4.71)$ & .04 & $0.30(-5.38-5.98)$ & .91 \\
\hline
\end{tabular}

Note: Multilevel structure applied in analyses to correct for nested structure of data within schools; $\mathrm{SB}=$ Sedentary Behavior; LPA = Light Physical Activity; MVPA = Moderate-to-Vigorous Physical Activity; C.I. = Confidence Interval

Table 7.3: Intervention components predicting change in percentage of recess time spent in SB, LPA, and MVPA after 12 months

\begin{tabular}{|c|c|c|c|c|c|c|}
\hline \multirow[t]{2}{*}{$(N=215)$} & \multicolumn{2}{|l|}{ SB } & \multicolumn{2}{|l|}{ LPA } & \multicolumn{2}{|l|}{ MVPA } \\
\hline & Std. Beta & $p$ & Std. Beta & $p$ & Std. Beta & $p$ \\
\hline Baseline \% in PA level & -.606 & $<.01$ & -.712 & $<.01$ & -.646 & $<.01$ \\
\hline Grade (ref $=6$ ) & .145 & .01 & -.058 & .30 & -.142 & .02 \\
\hline Gender (ref $=$ male $)$ & .217 & $<.01$ & -.116 & .03 & -.147 & .01 \\
\hline Ethnicity (ref = Dutch) & -.055 & .31 & -.075 & .15 & -.010 & .86 \\
\hline$\Delta$ in temperature $(\stackrel{\circ}{ } \mathrm{C})$ & .256 & .01 & -.178 & .05 & -.182 & .06 \\
\hline$\Delta$ in sun exposure & -.162 & .17 & .221 & .06 & -.025 & .84 \\
\hline$\Delta$ in precipitation & .069 & .44 & .019 & .82 & -.036 & .69 \\
\hline Number of PSIs & -.176 & .01 & .111 & .09 & .135 & .06 \\
\hline Number of SSIs & -.049 & .56 & .017 & .84 & -.021 & .81 \\
\hline Intensity of PSIs & -.564 & $<.01$ & .283 & $<.01$ & .477 & $<.01$ \\
\hline Project commitment & -.081 & .40 & -.014 & .88 & .047 & .62 \\
\hline$R^{2}$ & \multicolumn{2}{|c|}{$47.0 \%$} & \multicolumn{2}{|r|}{$50.1 \%$} & \multicolumn{2}{|r|}{$42.1 \%$} \\
\hline
\end{tabular}

Note: SB = Sedentary Behavior; LPA = Light Physical Activity; MVPA = Moderate-to-Vigorous Physical Activity; Bold = significant predictor of change caused by intervention component; PSI = Physical Schoolyard Intervention; SSI = Social Schoolyard Intervention

\section{DISCUSSION}

The aim of the current study was to test the 12-month effects of a multicomponent PA intervention at schoolyards on recess PA levels of $6^{\text {th }}$ and $7^{\text {th }}$ grade children in primary schools, combining GPS and accelerometry data. The multicomponent schoolyard intervention resulted in an increase in the time spent in LPA over time, but not in MVPA. The absence of an effect of the schoolyard intervention on MVPA is in contrast to most (multicomponent) schoolyard intervention studies that showed positive (MV)PA outcomes (Huberty et al., 2011; Ridgers et al., 2010b; Verstraete, Cardon, De Clercq, \& 
De Bourdeaudhuij, 2006). A possible explanation for our null-effect on MVPA could be found in the study duration. Most schoolyard intervention studies reported on shortterm effectiveness of interventions (Ickes et al., 2013), while we in line with our study purpose only studied long-term effects, i.e. 12 month effects. It might therefore be possible that we missed short-term effects of the current study. Results on short-term outcomes however, could be attributable to a 'novelty effect' (Stratton \& Mullan, 2005), while long-term effects are more likely to indicate sustained behavioral effects of interventions. The study design could also partially explain the lack of effect on MVPA. This study implemented a variety of schoolyard PA interventions in different types and intensities in different contexts.

The analytic approach testing the pooled effect of this multicomponent approach could have suppressed the effects of specific intervention components on MVPA. Therefore, we also introduced in-depth analyses of intervention schools to identify what type of intervention component (PSI vs SSI) most affected the changes in recess SB and PA over time. The main goal of the study was predicting sustainable change in children's PA levels during morning recess and environmental contexts in which interventions were implemented differed. This approach led to studying the effectiveness of the number of implemented stimuli per type of environment rather than evaluating specific intervention elements. PSIs predicted decreased time spent sedentary and an increased, although non-significant ( $p=.09$ and $p=.06$ respectively), proportion of time spent in LPA and MVPA at schoolyards at follow-up in these intervention schools. Next, we calculated the number of PSIs and assessed their effect. This approach showed that the more physical environmental stimuli were implemented, the larger the change in SB. The current project was originally designed to provide low-budget multicomponent PA schoolyard interventions, yet two schools succeeded in either raising more funds to increase the investment in the PSIs substantially (up to 70,000 euros) or implementing drastic changes at the schoolyard. These high intensity PSIs resulted in significantly larger increases in the time spent in LPA and MVPA than PSIs with a low intensity. SB decreased more over time after the implementation of high-intensity PSIs. Although PSIs with a higher intensity would be recommendable, the positive results for PSIs with a low intensity are also promising in terms of cost-effectiveness. It seems possible to successfully intervene on schoolyard SB with limited financial resources and non-drastic environmental adaptations.

No main effects were found for SSIs, which is likely to be a result of what was considered an SSI in this study. PA involvement and support by teachers (e.g. teachers playing together with children in recess) was previously shown to have positive associations with children's PA levels, especially among younger children (Brown et al., 2009; Cardon, Van Cauwenberghe, Labarque, Haerens, \& De Bourdeaudhuij, 2008; Huberty et al., 2011), but these types of interventions were not included in the current project. The lack of inclusion of these types of interventions was probably caused by the design of the project in which a substantial amount of autonomy was granted to the 
schools to implement interventions, which was hypothesized to enhance local support. Teachers showed less enthusiasm for interventions they were actively involved in themselves.

In this study, a prospective effect of the social environment was found in the moderating effect of school's commitment on the relation between PSIs and LPA and MVPA change over time. PSIs in highly committed schools showed better results in terms of LPA and MVPA. In contrast, PSIs in less committed schools seemed to have a negative effect on change in MVPA over time. A supportive school PA climate might therefore be essential for effective PSIs.

This was the first study to test the moderating effect of a school's commitment on the effect of schoolyard interventions on SB and PA. Embedding physical interventions in a supportive PA school climate is in line with the concept of ecological models (Kremers et al., 2006; Sallis et al., 2006), which supports the moderating effect found in this study. Although not directly comparable, the moderating effect of a supportive social environment has also been found in studies focusing on active school transport (Coombes, Jones, Page, \& Cooper, 2014; Faulkner, Zeglen, Leatherdale, Manske, \& Stone, 2014), and a supportive climate has also been reported to be beneficial in familybased childhood PA interventions (Kitzman-Ulrich et al., 2010; Skelton, Buehler, Irby, \& Grzywacz, 2012) as well as school-based obesity prevention programs (Lavelle, Mackay, \& Pell, 2012) and (Gubbels et al., 2011). In addition, recent reviews showed better results for interventions implemented in multiple settings (e.g. school and home) and focusing on multiple components (e.g. fixed equipment and green space) and multiple environmental domains (e.g. physical environment and policy environment) (Broekhuizen et al., 2014; De Vries, Hopman-Rock, Bakker, \& van Mechelen, 2009; Wang et al., 2015; Williams et al., 2013).

\section{Strengths, limitations, and recommendations for future research}

A major strength of the current study is the use of GPS and accelerometry. Without the combination of GPS and accelerometer data, we would have included all 10 pairs of intervention and control schools in the effect analyses, while in reality only 7 pairs were comparable in terms of exposure to the schoolyard during recess. Although we were able to adjust for this, it was striking that so many schools reported using the schoolyard during recess without actually doing so, especially since all schools reported a policy of children going outdoors during recess, irrespective of the weather conditions. Apart from data acquisition and processing techniques, the longitudinal quasi-experimental design of the study was also an advantage, as it enabled us to detect relationships between the environment (schoolyard) and children's behavior (PA). We also corrected for weather change scores between baseline and follow-up, correcting for potentially strong confounding variables such as temperature and precipitation. 
This study was also subject to some limitations. First, too few children wore a GPS device at baseline to make use of all the benefits of the combined GPS-accelerometry methodology; instead we used the GPS data to confirm if the schoolyard was used at all during recess. Although the lack of applying the combined GPS-accelerometry methodology (Jankowska et al., 2015) in this study, the use of GPS enhanced reliability of the results by testing whether children were exposed to environmental stimuli at schoolyards or not. The data included in the final analyses consisted of accelerometer data for the full period of scheduled recess time, rather than of time spent at schoolyards only. In addition, with respect to the implemented PA schoolyard interventions it should be addressed that in the primary schools' surroundings, additional PA stimulating activities were undertaken to support active school transportation and PA during leisure time (Van Kann et al., 2015a; Van Kann, Kremers, de Vries, de Vries, \& Jansen, 2016). Although such activities are unlikely to directly influence the relationship between PA schoolyard interventions and children's PA and SB during morning recess at schoolyards, a potential spill-over effect of these activities cannot be ruled out completely. Schools' commitment to the project might be considered as a proxy for these additional activities and was corrected for in the analyses. Moreover, the number of included days of valid PA data was limited. Children providing at least one valid day of data were included, which lowers the validity of the data. To account for this limitation forthcoming on the design of the study, additional inclusion criteria were formulated to enhance validity, such as providing valid daily PA data, defined as 480 minutes per day, instead of checking for valid recess PA data only. Besides, the thresholds for SB, LPA, and MVPA remain arbitrary and could have considerably influenced our findings regarding the proportion of time spent at the various PA levels (De Vries et al., 2009), hence they were kept the same during baseline and follow-up allowing to study change in PA levels. Furthermore, we lost some children at follow-up ( $\mathrm{N}=53 ; 6.7 \%)$ due to illness, moving to another school or refusal to continue participation. Over time, the number of children providing valid accelerometer data during recess decreased, i.e. compliance was highest during the first days of the data collection period at each school. Therefore, when planning future longitudinal studies among children using measuring equipment, especially studies combining accelerometers and GPS devices, it would be best to oversample at baseline. Finally, children in the intervention and control schools differed at baseline in the time spent in SB (significant trend) and MVPA. Since children in the intervention schools were both less sedentary and more moderately-to-vigorously physically active during recess at baseline, the effect of the interventions may have been underestimated. We tried to limit this confounding effect by correcting for the baseline proportion of time spent at different PA levels in the analyses. 


\section{CONCLUSION}

The multicomponent schoolyard PA intervention was effective in making children spend a larger proportion of recess time in light physical activity, which was most likely the result of a shift from sedentary behavior to light physical activity. Implementation of the intervention did not result in 12-month changes in moderate-to-vigorous physical activity. With regard to intervention components, implementing more physical schoolyard interventions contributed to less time spent in sedentary behavior in primary school children during recess. This effect was further enhanced by the intensity of physical schoolyard interventions and by implementation in a PA supportive school environment. 



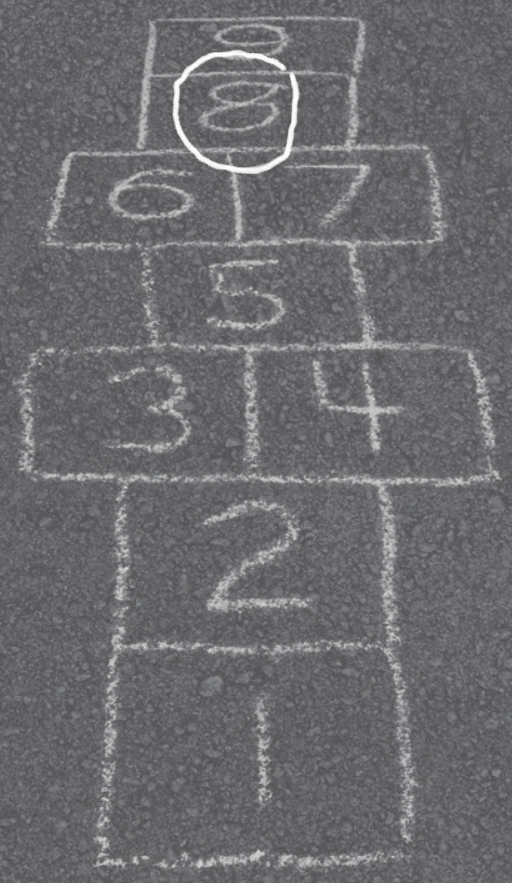

Chapter 8

The effect of a school-centered multicomponent intervention on daily physical activity and sedentary behavior in primary school children: the Active Living study

This chapter has been published as: Van Kann, D. H. H., Kremers, S. P. J., de Vries, N. K., de Vries, S. I. \& Jansen M. W. J. (2016). The effect of a school-centered multicomponent intervention on daily physical activity and sedentary behavior in primary school children: the Active Living study. Preventive Medicine, 89, 64-69. 


\section{ABSTRACT}

The aim of the current study was to examine the effectiveness of a school-centered multicomponent PA intervention, called 'Active Living', on children's daily PA levels.

A quasi-experimental design was used including 9 intervention schools and 9 matched control schools located in the Netherlands. The baseline measurement took place between March-June 2013, and follow-up measurements were conducted 12 months afterwards. Accelerometer (ActiGraph, GT3X+) data of 520 children aged 8-11 years were collected and supplemented with demographics and weather conditions data. Implementation magnitude of the interventions was measured by keeping logbooks on the number of implemented physical environmental interventions (PEIs) and social environmental interventions (SEIS). Multilevel multivariate linear regression analyses were used to study changes in sedentary behavior (SB), light physical activity (LPA) and moderate-to-vigorous physical activity (MVPA) between baseline and followup. Finally, effect sizes (ESs) were calculated using Cohen's $d$.

No pooled effects on PA and SB were found between children exposed and not exposed to Active Living after 12 months. However, children attending Active Living schools that implemented larger numbers of both PEIs and SEls engaged in 15 more minutes of LPA per weekday at follow-up than children in the control condition ( $E S=0.41 ; p<.05)$. Moreover, children attending these schools spent less time in SB at follow-up ( $E S=0.33$ ), although this effect was non-significant. No significant effects were found on MVPA.

A school-centered multicomponent PA intervention holds the potential to activate children, but a comprehensive set of intervention elements with a sufficient magnitude is necessary to achieve at least moderate effect sizes. 


\section{INTRODUCTION}

Increased physical inactivity (Kohl et al., 2012) is a global threat to health and contributes to multiple diseases throughout the lifespan (Andersen et al., 2006; Ekelund, Luan, Sherar, \& et al., 2012; Saunders et al., 2014). Childhood physical activity (PA) and sedentary patterns track into adulthood (Parsons et al., 1999; Singh et al., 2008; Telama, 2009), indicating a necessity to stimulate physical activity at an early age and for early prevention of sedentary behaviors (SB). Low PA and high levels of SB are independent risk factors for health, rather than opposite behaviors in a continuum, and have their own independent determinants (Pearson et al., 2014; Saunders et al., 2014). Primary schools are suitable settings for preventive health activities, since children spend a substantial amount of time in schools during weekdays (Story et al., 2009). A healthy, PA-supportive school environment has the potential to influence childhood PA levels (Escalante et al., 2014; Ridgers, Salmon, Parrish, Stanley, \& Okely, 2012b). Despite their important position in reaching out to and educating children, schools are only one setting within a broader system in which children operate (Koplan et al., 2005; Pate, Wang, Dowda, Farrell, \& O'Neill, 2006a). Another setting affecting childhood PA patterns is the neighborhood children reside in. A PA-supportive neighborhood is positively associated with children's outdoor play behavior (Aarts et al., 2012). For instance, social safety and traffic safety affect both outdoor play and active transport to school (Carver et al., 2008b; De Vries et al., 2010; Timperio et al., 2006). A third setting children operate in is the home environment. Parental behaviors and decisions influence children's PA and SB, especially those of young children (Carver et al., 2013; Faulkner et al., 2010). Interactions between activity-inducing physical environmental factors (e.g. the availability of playgrounds in the neighborhood) and social environmental factors (e.g. supportive parental practice towards child's PA behavior) in each setting are assumed to strengthen each other's impact on child behaviors (Gubbels et al., 2014). In other words, the implementation of supportive actions within multiple types of environment, i.e. physical and social, is assumed to have a synergetic effect on child's PA (Gubbels et al., 2011).

Many school-based PA interventions have been implemented (Broekhuizen et al., 2014; Dobbins et al., 2013), but studies found that their effectiveness differs greatly. Generalizability of these studies is limited due to the variety of types, numbers and intensities of interventions. The challenge of generalizing results may be met by performing an implementation-focused process evaluation for each type of environmental intervention (Elder et al., 1994). In addition, school-based interventions tend to focus only on the school setting, e.g. schoolyards, and pay little or no attention to other settings such as the local neighborhood and the home environment. In order to connect the school setting with other settings, the Active Living study has been developed and implemented as a school-centered PA intervention (Van Kann et al., 2015a). To increase PA and decrease SB in primary school children, the Active Living 
study implemented multicomponent PA interventions at or around school to enhance PA in three domains; (1) in school, (2) during active school transport, and (3) during leisure time (Van Kann et al., 2015a). In all three PA domains, both physical and social environmental interventions (PEIs and SEIs) were implemented.

The aim of the current study was to test the effectiveness of 'Active Living' in terms of children's daily PA and SB levels, while taking exposure to PEIs and SEIs into account. We hypothesized that children who were exposed to more PEls and SEls would increase their PA levels and decrease their SB more than children who were less exposed to these interventions and than children who were not at all exposed to Active Living interventions (control condition).

\section{METHODS}

\section{Study design and protocol}

To test the effectiveness of the Active Living study, a longitudinal, quasi-experimental design was used. The study was conducted among 21 primary schools located in deprived areas (low SES) in the Southern-Limburg region of the Netherlands. A detailed description of the study design, methods, and implementation strategies has been published elsewhere (Van Kann et al., 2015a). The Active Living study received ethical approval from the Medical Ethics Committee of the University Hospital Maastricht (METC 12-4-077) and is registered as a Current Controlled Trial (ISCTRN25497687).

\section{Study sample}

For the purpose of the current study, grade 6 and 7 children (aged 8-11 years) from 21 schools were included; 10 Active Living intervention schools and 11 matched control schools. In one case, an intervention school was matched to two control schools, due to unexpected environmental changes in the control school between the feasibility test in the autumn of 2012 and the baseline measurement in the spring of 2013. After the baseline measurement, no further changes were noticed in this control school, making both control schools eligible for inclusion in this study. The baseline measurement (TO) took place between March and June 2013. The follow-up measurement was conducted between March and June 2014 (T1). Schools consented filling out a questionnaire in the classroom. At baseline, all children ( $N=1343)$ filled out a questionnaire, and 791 of them were fitted with an accelerometer (58.9\%). Written parental consent to wear an accelerometer, however, was obtained prior to the baseline measurement. To correct for potential seasonal effects (Rich, Griffiths, \& Dezateux, 2012), every interventioncontrol school pair was assessed on the same dates. Moreover, a minimum number of 10 children per school providing valid accelerometer data at both baseline (TO) and 
follow-up (T1) was defined as an inclusion criterion. If a school in a pair (interventioncontrol) did not meet the inclusion criterion, the pair was deleted from the data analyses.

\section{Intervention packages}

Implemented PEIs and SEls were all intended to stimulate PA or reduce SB (Table 8.1). An overview of all activities is provided per school in appendix 3 (Table S8.1). All interventions were implemented at school or within an 800 meter radius around the school. In the Netherlands, the majority of the primary school children lives within 800 meter distance from school (CBS, 2014). No additional activities were implemented at control schools during the intervention period. A working group at each intervention school was responsible for choosing, designing and implementing intervention elements. All working groups received an intervention budget of 2,000 euros at the start of the project. Although sharing an overall scope, the intervention packages could differ in magnitude and design across schools. Working groups defined local needs and designed interventions accordingly. Implementation magnitude was measured by keeping logbooks on the number of implemented PEIs and SEIs. The number of implemented interventions was dichotomized (high/low) using median split (PEI $\geq 4=$ high; SEl $\geq 6$ =high), and a 'set of interventions' variable was defined as 'high magnitude set' if both the dichotomized PEls and SEls variable were high. Other combinations were defined as 'low magnitude set', while the number of implemented interventions in control schools was set to zero.

\section{Measures}

Primary outcome variables of this effectiveness study were changes in PA and SB on weekdays after 12 months. PA data were collected using accelerometers (ActiGraph GT3X+; $30 \mathrm{~Hz}, 10$-second epochs) and data were processed using ActiLife version 6.10.4 (ActiGraph, Pensacola, USA). Wear time validation was assessed using Choi's classification criteria (Choi, Liu, Matthews, \& Buchowski, 2011) and minimal wear time was defined as 480 minutes per day during waking hours between 6am and 11pm (Jago et al., 2013). In view of the focus of the intervention packages of the Active Living study (see Appendix 3; Table S8.1), only weekdays were included in the analyses. The first day of measurement was excluded to prevent reactivity (Dössegger et al., 2014). Valid weekdays, with a minimum of 1 after excluding the first measurement day, were aggregated per child to be included in the statistical models. Evenson's cut-offs (Evenson et al., 2008) were used to classify PA data into sedentary behavior (SB; $\leq 100$ counts-per-minute), light PA (LPA; 101 - 2295 counts-per-minute), and moderate-tovigorous PA (MVPA; $\geq 2296$ counts-per-minute). Time spent in SB, LPA, and MVPA was 
divided by wear time to calculate a proportion of time per day spent in SB, LPA, and MVPA, respectively.

Demographic variables were collected using questionnaires completed by the children. Child's age was calculated by subtracting the self-reported date of birth from the first date of measurement at baseline. Other demographic variables were gender and ethnicity (based on country of birth of the child and both parents). Mean temperature, sun exposure and precipitation between $6 \mathrm{am}$ and $11 \mathrm{pm}$ were calculated for every single measurement day before aggregating data (Royal Dutch Meteorological Institute (KNMI)). Individual change scores $(\Delta)$ for weather condition between baseline and follow-up measurement were calculated by subtracting the values of aggregated baseline data from those of aggregated follow-up data.

Table 8.1: Examples of implemented interventions

\begin{tabular}{lll}
\hline PA Domain & $\begin{array}{l}\text { Example of Physical Environmental } \\
\text { Intervention (PEI) }\end{array}$ & $\begin{array}{l}\text { Example of Social Environmental } \\
\text { Intervention (SEI) }\end{array}$ \\
\hline 1. PA in school & $\begin{array}{l}\text { Providing (loose and fixed) play } \\
\text { equipment at schoolyard; Providing } \\
\text { playground markings }\end{array}$ & $\begin{array}{l}\text { Introducing sports clinics during recess; } \\
\text { Specifying the potential activating role } \\
\text { a teacher holds in recess }\end{array}$ \\
$\begin{array}{l}\text { 2. PA during active school } \\
\text { transport }\end{array}$ & $\begin{array}{l}\text { Creating safer parking situation around } \\
\text { school (school zones); Providing bicycle } \\
\text { racks }\end{array}$ & $\begin{array}{l}\text { Initiating a walk/cycle-to-school-day; } \\
\text { Running an AT sticker-competition }\end{array}$ \\
3. PA during leisure time & $\begin{array}{l}\text { Upgrading local playgrounds; } \\
\text { Redesigning local playgrounds }\end{array}$ & $\begin{array}{l}\text { Establishing out-of-school activity } \\
\text { program; Connecting local sports clubs } \\
\text { with schools }\end{array}$ \\
\hline
\end{tabular}

\section{Statistical analyses}

Statistical analyses used SPSS version 21.0 (IBM, USA) and significance was defined as $p$ $<.05$. Various multilevel linear regression analyses were used to study the effectiveness of interventions regarding change in PA and SB. We first tested the pooled effectiveness of the experimental versus control condition. For this purpose, we constructed three two-level regression models with the proportion of time spent in SB, LPA, and MVPA, respectively, as dependent outcome variables while correcting for potentially confounding factors such as demographic variables and weather conditions. School was entered as a level-one independent variable, while baseline proportions of time spent in SB, LPA, and MVPA, respectively; gender; age; ethnicity; change scores for temperature, sun exposure, and precipitation; and condition (intervention/control) were entered as independent variables at level two.

Subsequently, we restructured our models by replacing the condition variable by the dichotomized PEI variable, setting the number of PEls at control schools at zero. A third model contained the same predictors of change, but included the dichotomized SEI variable. The final model included the same predictors as previous models, but now included the 'set of interventions' variable. These three models were corrected for the 
similar confounding factors as included in the pooled effectiveness analyses. Finally, effect sizes (ESs) were calculated using Cohen's $d$ (Cohen, 1988) and ESs were interpreted using Lipsey's cut-off values, indicating a small effect for ESs $\leq 0.32$, a moderate effect for ESs between 0.33-0.55 and a large effect for ESs $\geq 0.56$ (Lipsey, 1990).

\section{RESULTS}

\section{Demographics and intervention implementation}

In total, nine (intervention-control) school pairs were included in the analyses. One school pair was excluded for not meeting the criterion on the minimum number of children providing valid accelerometer data. At the 18 included schools, 520 children (74.2\%) provided valid accelerometer data both at TO and T1. Fifty-eight percent of these children were enrolled at an intervention school. Slightly more girls (56\%) than boys provided valid data, and the mean age of children at baseline was 10.1 years. The majority of the children had been born in the Netherlands ( $N=490 ; 94 \%$ ) (Table 8.2).

At baseline, children spent $65.4 \%$ of their time in SB, whereas the proportions of time spent in LPA and MVPA were $27.4 \%$ and $7.2 \%$, respectively with no significant differences between children attending intervention and control schools. The proportion of daily time spent in MVPA corresponds to 56.3 minutes (SD \pm 22.6 ). After 12 months, children spent on average 2.2\% (SD \pm 7.4$)$ more daily time in $\mathrm{SB}$, while they spent $1.7 \%(S D \pm 5.5)$ less daily time in $\mathrm{LPA}$ and $0.5 \%(\mathrm{SD} \pm 1.2)$ less in MVPA. These proportions correspond to 17.0 additional minutes of SB a day and 13.2, and 3.9 fewer minutes of LPA and MVPA a day, respectively. At follow-up, the temperature was on average 0.4 degrees Celsius higher than at T0. The sun exposure had increased by an additional 2.6 hours a day at T1, and on average 0.6 fewer hours of precipitation a day were measured at $\mathrm{T} 1$.

On average, 4.1 PEls were implemented at the intervention schools, ranging from a minimum of 2 to a maximum of 8 per school. The average number of implemented SEIs was 4.8, ranging from 3 to 10 per intervention school (Table 8.2). 
Table 8.2: Characteristics of study population

\begin{tabular}{|c|c|c|c|}
\hline & Total $(N=520)$ & Intervention $(\mathrm{N}=301)$ & Control $(\mathrm{N}=219)$ \\
\hline \multicolumn{4}{|l|}{ Demographics } \\
\hline Age; Mean (SD) & $10.13(.69)$ & $10.13(.68)$ & $10.13(.70)$ \\
\hline \multicolumn{4}{|l|}{ Gender } \\
\hline Boys; N (\%) & $231(44.4)$ & $143(47.5)$ & $88(40.2)$ \\
\hline Girls; N (\%) & $289(55.6)$ & $158(52.5)$ & $131(59.8)$ \\
\hline \multicolumn{4}{|l|}{ Ethnicity } \\
\hline Dutch; N (\%) & $490(94.2)$ & $282(93.7)$ & $208(95.0)$ \\
\hline Non-Dutch; N (\%) & $30(5.8)$ & $19(6.3)$ & $11(5.0)$ \\
\hline \multicolumn{4}{|l|}{ PA levels at baseline } \\
\hline $\mathrm{SB} ; \%(\mathrm{SD})$ & $65.4(6.7)$ & $65.7(7.1)$ & $64.9(6.2)$ \\
\hline LPA; \% (SD) & $27.4(5.2)$ & $27.0(5.5)$ & $27.9(4.8)$ \\
\hline MVPA; \% (SD) & $7.2(2.8)$ & $7.3(2.8)$ & $7.2(2.9)$ \\
\hline CPM (X-axis); mean (SD) & $528.1(197.9)$ & $521.9(191.9)$ & $536.6(206.0)$ \\
\hline CPM (Vector Magnitude); mean (SD) & $1007.5(291.6)$ & $999.6(289.1)$ & $1018.3(295.4)$ \\
\hline \multicolumn{4}{|l|}{ Implemented interventions } \\
\hline Physical Environment (PEI); mean (SD) & & $4.1(1.8)$ & Not applicable \\
\hline Social Environment (SEI); mean (SD) & & $4.8(1.9)$ & Not applicable \\
\hline
\end{tabular}

Predicting change in SB, LPA, and MVPA by intervention condition, demographics, and weather conditions

Overall, a multilevel regression model including the condition variable, i.e. intervention or control condition, showed no significant pooled effect on time spent in either of the three PA categories (Table 8.3). Baseline proportion of time spent in each activity category strongly predicted the proportion of time spent in the same activity category after 12 months. Moreover, SB increased and LPA and MVPA decreased between TO and T1. Being older at baseline and being a girl significantly predicted increased time spent in SB and decreased time spent in MVPA and LPA after 12 months. Neither ethnicity nor the difference in weather conditions between the two time points was a significant predictor of change in time spent in SB, LPA or MVPA (Table 8.3).

\section{Predicting change in SB, LPA, and MVPA by type of intervention}

As regards the number of PEls, a trend towards a significant positive effect $(p=.08)$ on the time spent in LPA, with a medium effect size (ES=0.34), was found for implementing a large number $(\geq 4)$ of PEls. As regards the other two PA classifications, no statistically significant effects of implementing PEls were found. A small non-significant trend with a medium effect size ( $E S=0.34$ ) towards an increase in LPA was found in the group that implemented a large number $(\geq 6)$ of SEls. Neither PEIs nor SEls were found to have effects on MVPA, although estimates showed a slight (non-significant) decrease in MVPA over time. All models yielded outcomes comparable to those in the condition 
model with regard to covariates that predicted the changes in time spent in SB, LPA, and MVPA (Table 8.3).

As regards the 'set of interventions' variable (combining PEIs and SEIs), a significant positive effect on LPA, with a medium effect size (ES=0.41), was found for the high magnitude set of interventions. Children attending these schools spent on average 15.4 minutes more in LPA during a weekday after 12 months, which reflects a medium effect size (Figure 8.1). A non-significant decrease in SB was found, with a medium effect size (ES=0.33). This is likely the result of a switch from SB to LPA, based on the estimates in the model (Table 8.3). No significant effects on changes in SB, LPA, or MVPA were found in the low magnitude groups.

Table 8.3: Predictors of share of time spent in SB, LPA, and MVPA after 12 months

\begin{tabular}{|c|c|c|c|c|c|c|}
\hline \multirow[t]{2}{*}{$(N=520)$} & \multicolumn{2}{|l|}{ SB } & \multicolumn{2}{|l|}{ LPA } & \multicolumn{2}{|l|}{ MVPA } \\
\hline & Beta (95\%C.I.) & $\mathrm{p}$ & Beta (95\%C.I.) & $\mathrm{p}$ & Beta (95\%C.I.) & $\mathrm{p}$ \\
\hline Baseline \% PA & $.38(.30-.46)$ & $<.01$ & $.39(.32-.47)$ & $<.01$ & $.36(.28-.45)$ & $<.01$ \\
\hline Age & $1.09(.33-1.85)$ & $<.01$ & $-.65(-1.22--.08)$ & .02 & $-.43(-.77--.09)$ & .01 \\
\hline Gender (male) & $-1.98(-3.03--.93)$ & $<.01$ & $.76(-.02-1.54)$ & .06 & $1.25(.77-1.74)$ & $<.01$ \\
\hline Ethnicity (Dutch) & $.09(-2.73-2.90)$ & .95 & $-.04(-2.13-2.05)$ & .97 & $-.00(-1.25-1.25)$ & .99 \\
\hline$\Delta$ Temperature (으) & $.01(-.01-.03)$ & .52 & $-.01(-.02-.01)$ & .44 & $-.00(-.01-.01)$ & .87 \\
\hline$\Delta$ Sun exposure & $-.23(-.69-.22)$ & .31 & $.17(-.15-.49)$ & .33 & $.06(-.15-.28)$ & .54 \\
\hline$\Delta$ Precipitation & $.18(-.52-.88)$ & .60 & $-.23(-.74-.27)$ & .38 & $.03(-.30-.35)$ & .87 \\
\hline Condition (Intervention) & $.02(-2.17-2.21)$ & .99 & $.58(-.93-2.10)$ & .43 & $-.58(-1.64-.49)$ & .26 \\
\hline \multicolumn{7}{|l|}{ Number of PEls } \\
\hline Low & $0.93(-1.61-3.47)$ & .44 & $-.21(-1.86-1.45)$ & .79 & $-.71(-2.04-.59)$ & .25 \\
\hline High & $-1.23(-4.06-1.60)$ & .37 & $1.67(-.19-3.52)$ & .08 & $-.35(-1.81-1.11)$ & .61 \\
\hline \multicolumn{7}{|l|}{ Number of SEls } \\
\hline Low & $1.10(-1.20-3.40)$ & .31 & $-.14(-1.74-1.47)$ & .85 & $-.92(-2.15-.31)$ & .13 \\
\hline High & $-1.51(-4.08-1.07)$ & .23 & $1.67(-.14-3.48)$ & .07 & $-.10(-1.45-1.26)$ & .88 \\
\hline \multicolumn{7}{|l|}{ Package PEls * SEls } \\
\hline Low & $1.01(-1.16-3.18)$ & .33 & $-.08(-1.59-1.42)$ & .91 & $-.87(-2.04-.30)$ & .13 \\
\hline High & $-2.04(-4.88-.80)$ & .15 & $2.06(.08-4.04)$ & .04 & $.05(-1.47-1.57)$ & .95 \\
\hline
\end{tabular}

Note: Multilevel structure applied in analyses to correct for nested structure of data within schools; Estimates covariates are based model with condition, covariates did not change substantially in other models; $\mathrm{SB}=$ Sedentary Behavior; LPA = Light Physical Activity; MVPA = Moderate-to-Vigorous Physical Activity; C.I. = Confidence Interval 


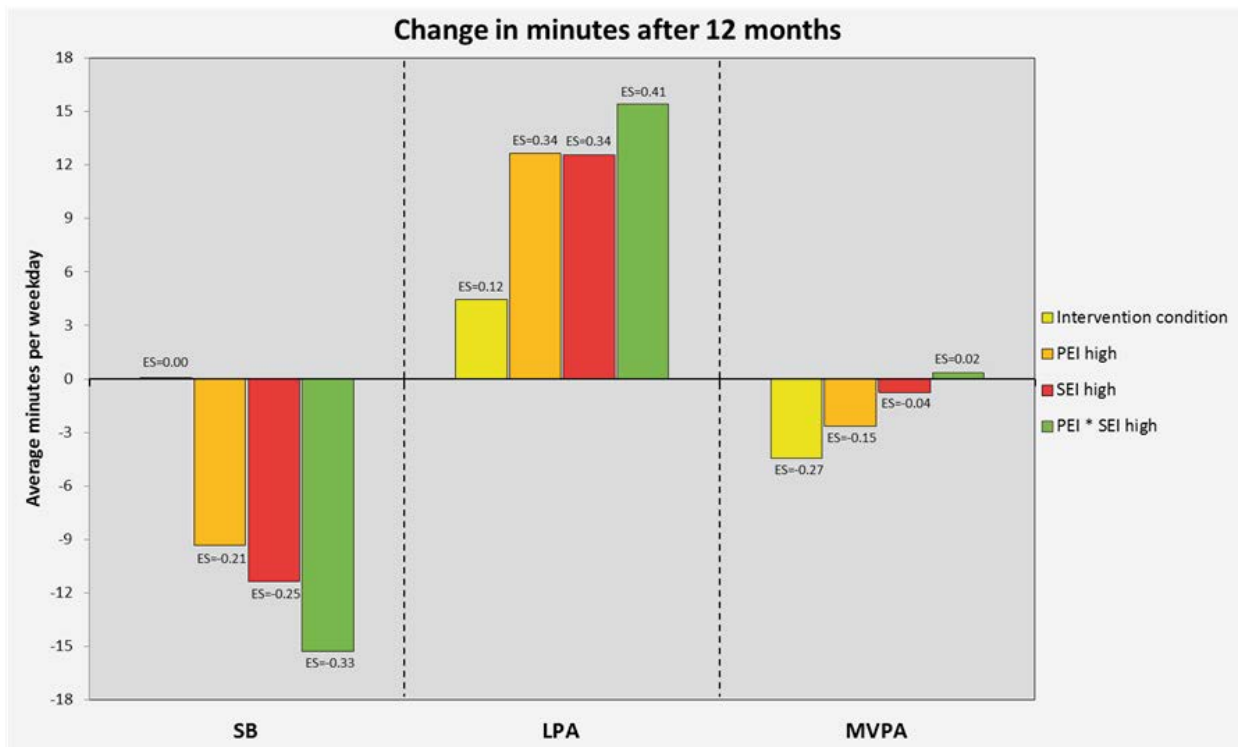

Figure 8.1: Intervention effects on SB, LPA, and MVPA expressed in average minutes per weekday and Cohen's $d$

\section{DISCUSSION}

The aim of the current study was to test the effectiveness of the Active Living intervention on children's time spent in SB and PA. Children exposed to Active Living did not differ from those at control schools with regard to PA and SB after 12 months.

In line with results from the SPEEDY study in the UK (Corder, van Sluijs, Ekelund, Jones, \& Griffin, 2010), we found a general reduction in PA and increase in SB at followup. This reduction could most likely be explained by the increase in age. In addition, female gender predicted a decline in PA and increase in SB. Older children and (especially) girls became less active over time, in line with previous studies, which often found that girls during early adolescence tend to reduce their time spent in PA (Craggs, Corder, van Sluijs, \& Griffin, 2011). Exposure to the Active Living project did not prevent this reduction in PA.

A large variation in the number of implemented interventions per school led to different levels of exposure to intervention elements of the children. A more in-depth analysis of the PEIs and SEls showed that if a substantial number of interventions was implemented, the proportion of time spent in LPA increased non-significantly (Figure 8.1), although this increase indicated a trend towards significance $(p<.10)$. In line with Gubbels' recommendations (Gubbels et al., 2014), the activating effect of these types of intervention was optimal when a combination of multiple PEls as well as SEls were implemented. Children exposed to a substantial number of both types of environmental 
interventions spent 15 minutes more time a day in LPA and 15 minutes less in SB than children in the control condition. Although the reduction in time spent in SB was not significant, the effect size was medium. This finding underlines the importance of implementing a comprehensive package, including multiple types of environmental interventions, rather than 'single shots', to increase PA levels and subsequently reduce overweight (Kriemler et al., 2011; Sobol-Goldberg, Rabinowitz, \& Gross, 2013). The set of interventions, although small in terms of size and budget, resulted in a substantial increase in the level of LPA and a decrease of time spent in SB. Basically, this may be reformulated as 'do it well or don't do it at all'. The recommendation to implement sufficient numbers of both PEls and SEls could imply that there might be a certain threshold effect of the number of combined stimuli that should be implemented to activate children in primary school interventions. Further research on this threshold hypothesis is needed.

Irrespective of the level of specification of effect analyses, i.e. taking into account the level of exposure, no effect was found on MVPA. Although this study was initially designed to activate children, the lack of an effect on MVPA contradicts findings of some other (setting-specific) school-based PA interventions (Kriemler et al., 2011; Ridgers et al., 2012a). There might be several explanations for the lack of effect on MVPA. Firstly, we studied MVPA on a daily basis and as a proportion of time per day spent in MVPA. Small changes resulting from the interventions within a short period of time, such as an increase in MVPA during recess periods, could be obscured or compensated by activities over the rest of the day, as has been suggested by the 'activitystat' hypothesis (Rowland, 1998). In line with this hypothesis, Metcalf and colleagues (2012) found a limited effect of PA interventions on whole-day MVPA. Combined time-specific and location-specific analyses would potentially be helpful to guide future intervention development and evaluation (Brooke, Corder, Atkin, \& van Sluijs, 2014). The limited intensity of the implemented PEIs and SEIs specifically aimed at MVPA (e.g. optimizing PE classes) might be another possible explanation for the lack of effects regarding this outcome. Moreover, the implemented interventions targeted mostly the school setting and hardly (if at all) concerned other MVPA-affecting settings such as the local neighborhood (Klinker et al., 2014b; Van Sluijs et al., 2011), leading to less exposure to PEIs and SEIs in the leisure time domain. Additionally, the home environment received little attention. The home environment has been reported to be an important setting for health-promotion activities, especially for younger children (Van Sluijs et al., 2011). The suboptimal reach of other settings than the school setting might also explain the lack of pooled effectiveness of Active Living. Focusing on the child system as a whole might be more effective in changing health behavior (Thelen \& Smith, 2006). 


\section{Strengths and limitations}

The quasi-experimental design with matched schools supplemented with an implementation-focused process evaluation is a major strength of this study. We were able to test the effectiveness in terms of children's PA levels of a number of PEls and SEls that shared the same scope, but differed in magnitude and design. The objectively assessed PA levels collected in the same season, and the matching of measurements in the same week, were also advantages of this study, as were the number of study sites and children enrolled in the Active Living project.

Using a synergetic physical and social environmental intervention approach was helpful in assessing the magnitude of PA interventions that showed the potential to enhance PA and reduce SB, but simultaneously limited the opportunities to provide concrete suggestions on the effectiveness of specific interventions. In addition, this approach refers only to the number of intervention elements regardless of the quality of their implementation. Furthermore, our study results related to effects of PA and SB during weekdays. It is unclear whether these results are sustained during weekends and in the longer term. According to our protocol, children wore accelerometers for at least 5 consecutive days, including a weekend. Fidelity to the wear protocol during weekends was, however, low. On the other hand, most intervention activities were implemented in the immediate school environment, suggesting that change is more likely to occur during weekdays compared to weekends.

\section{CONCLUSION}

Physical and social environmental PA interventions at or around schools hold the potential to activate children on a daily basis. However, the present study showed that multiple physical and social environmental PA stimuli are needed to change the time spent in LPA. The results indicate the need to intensively address multiple types of environmental factors rather than focus on a few specific elements of the children's environment. 


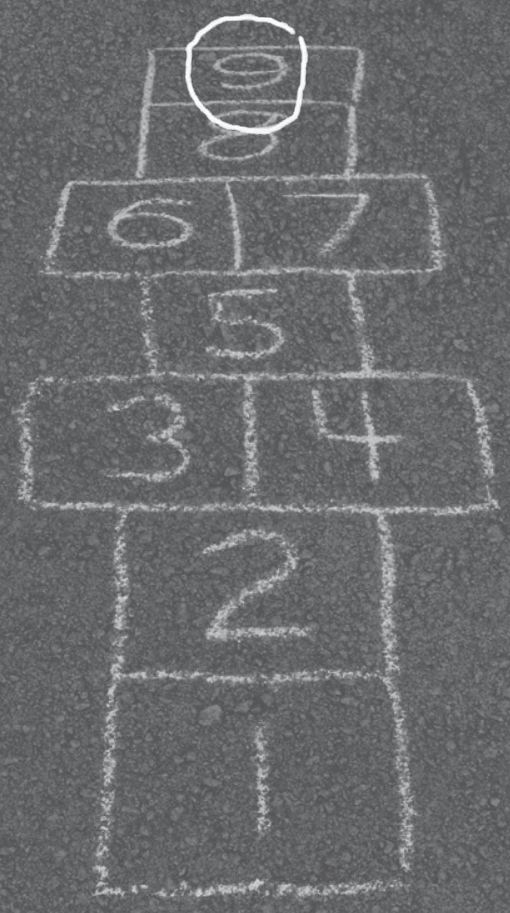

Chapter 9

General discussion 


\section{INTRODUCTION}

The primary aim of the work reported on in this thesis was to study the development, implementation, and evaluation of a multicomponent school-centered intervention to increase the physical activity (PA) and reduce sedentary behavior (SB) of primary school children aged 8-12 years in the Southern Limburg region of the Netherlands. Additional studies were performed to examine the relationship between environment and PA behavior. These studies focused on active school transportation (AST), PA during leisure time, and PA at schoolyards.

This chapter discusses the main findings of the seven studies described in this thesis. It discusses methodological issues faced in these studies, in order to facilitate the interpretation of the results. This is followed by theoretical considerations, and recommendations for practice and for future research. The chapter ends with a general conclusion.

\section{MAIN FINDINGS}

\section{Development and implementation of the Active Living project}

Chapter 2 provides an overview of the development and implementation of the Active Living project and the quasi-experimental design of the evaluation study. Active Living was a school-centered, multicomponent PA intervention focusing on three conceptual PA domains: PA during AST, PA during leisure time, and PA at schoolyards. Starting point of the Active Living project was to establish a sustainable PA-supportive environment. Applying a combined top-down and bottom-up approach proved to be feasible and resulted in the implementation of multiple PA interventions both in the physical and social environment. The responsibility to determine the schools' PA priorities for children was given to working groups that were established at each intervention school (bottom-up). Working groups consisted of at least a health promotor (from the regional Public Health Services), teachers, parents and municipal sports officers, and were given a relatively large degree of autonomy to prioritize the focus of PA interventions. The autonomous working groups were supported by the program development team, consisting of researchers and health promoters, to successfully develop/adopt and implement PA interventions in the schools (Ryan \& Deci, 2000). Evidence-based working was stimulated by a close collaboration between the working groups in schools and a research group which 'fed' the working groups with scientific evidence and good practices (top-down; see Figure 2.1). Based on our study, applying the combined topdown and bottom-up approach is recommended to enhance local support for environmental interventions, such as Active Living. 


\section{Active school transportation}

Chapter 3 describes a cross-sectional study on the association between the physical school environment and the use of AST among children aged 5 to 12 years. Although the Dutch built environment is recognized worldwide for its excellent infrastructure opportunities for active transportation, such as the presence of bicycle lanes, we found that one in three children did not use AST. In this study we used Pikora's theoretical framework to subdivide environmental characteristics into four clusters: (1) destination, (2) safety, (3) aesthetics, and (4) functional cluster (Pikora et al., 2003). We found that environmental clusters were not associated with AST among the youngest children (aged 5-9 years, grades 1-5). For the children aged 9-12 years (grades 6-8), the aesthetics cluster was associated with the use of AST. Aesthetic aspects, such as littering, were associated with the absence of AST for the children aged 9-12 years. The safety cluster was not significantly associated with AST, which might be explained by hierarchical needs for using AST (Alfonzo, 2005). Safety could be considered a precondition (higher hierarchical need), which, once fulfilled, might no longer predict the active traveling behavior. Local aesthetics could then be regarded as a lower hierarchical need for the use of active transportation.

Chapter 4 examines the association between the physical school environment and AST in children aged 8-11 years in more detail, including social environment constructs, i.e. parental PA practices (Davison et al., 2011; Gattshall et al., 2008) and parental active transportation routines (PATRns) as associates of AST. PATRns were also tested as a potential moderator of the associations between the physical environment and AST. A shorter distance to school, higher age of the child, and greater traffic safety were found to be positive associates of AST. PATRns were positively associated with a larger number of days travelling to school of the child by means of active transportation, and in addition moderated the association between environmental factors and AST. For children raised in families in which the parents use active transportation frequently (high-PATRns), distance between home and school, though generally indicated as the most important determinant of AST (Larouche, 2015; Timperio et al., 2015), was a substantially weaker barrier to AST than for children raised in low-PATRns families. For children living in high-PATRns families living close to school, distance to school was not found to be a barrier to AST, whereas distance was still a barrier for children raised in low-PATRns families. Accordingly, one could consider encouraging parents to walk and cycle in order to stimulate children's AST.

\section{PA during leisure time}

Chapter 5 addresses the after-school PA of children aged 8-11 years. This study particularly focused on the playability of school environments and its association with children's afterschool PA. Playability of the school environment was studied by observing the number of 
playgrounds and assessing their characteristics (i.e. size, accessibility, opening hours, maintenance, availability of playing devices and age-appropriateness) located in an $800 \mathrm{~m}$ crow-fly buffer around primary schools. In our sample, $70 \%$ of the children lived within the observed school environment (800 $\mathrm{m}$ radius) for which the playability score was calculated and their PA was assumed to be more influenced by the playability of the school environment than children living outside this area. Concurrent activities during leisure time, such as sports participation, and seasonal effects (e.g. daylight) might also affect the association between playability and PA (Goodman et al., 2014). Therefore, we studied the association between playability and PA for specific home-school distance categories (spatial filters) and after-school periods (time filters). Children attending schools located in more playable areas spent more time in light PA (LPA) and moderate-to-vigorous PA (MVPA) immediately after school ended, up to $6 \mathrm{pm}$. The association attenuated at later hours, and faded out after $6 \mathrm{pm}$. Distance specific analyses showed a positive association between playability and LPA for children living within $400 \mathrm{~m}$ from their school, while the association between playability and MVPA remained positive up to a distance of $800 \mathrm{~m}$. No association between playability and PA for children living beyond these $400 \mathrm{~m}$ and $800 \mathrm{~m}$ radius buffered zones. The associations we found underline the importance of the availability of well-designed playgrounds in children's school environment. In addition, the time and spatial filters we applied can be used to inform health promotors on how to implement their PA-promotion activities in the right place and at the right time.

\section{PA at schoolyards}

Chapter 6 focuses on schoolyard characteristics that were associated with moderate-tovigorous PA (MVPA) and sedentary behavior (SB) in children aged 8-11 years. We investigated this using an innovative method combining accelerometry, Global Positioning System (GPS) and Geographical Information System (GIS) data (Jankowska et al., 2015; Klinker et al., 2015). On average, almost 20\% of daily MVPA took place at schoolyards. In addition, we found that children spent 54 minutes per school day at the schoolyards, and approximately one fifth $(9 \mathrm{~min}$ ) of this time was spent in MVPA. The proportion of time spent in MVPA emphasizes the importance of schoolyards for MVPA stimulation. Boys were significantly more active at schoolyards than girls, which was in line with activity levels during the rest of the day and previous studies. The presence of fixed equipment, such as soccer goals and high bars, was associated with more time spent in MVPA and less time spent in SB. Surprisingly, the presence of schoolyard markings was associated with a lower amount of time spent in MVPA by children aged 8 to 11 years, which could possibly be explained by the age-appropriateness or inappropriateness of the design of the markings. We found a positive influence of the presence of green spaces at the schoolyard on the time spent sedentary. This may have been caused by the fact that observed schoolyard green spaces mostly had an aesthetical function, e.g. consisting of hedges or trees, whereas PA-supportive green 
spaces such as soccer fields were lacking in the observed schoolyards. As regards the social environment, we found that activities in the schoolyard initiated by children themselves were associated with less MVPA and more SB compared to activities initiated by teacher involvement, whereas the presence of schoolyard regulations concerning ball games areas was associated with more MVPA and less SB, as were extended opening hours of the schoolyard. These associations indicate opportunities for schools to stimulate PA and reduce SB while being at the schoolyard either during school operating hours or before and after that.

Chapter 7 describes the effects of a multicomponent PA schoolyard intervention on the activity levels of children aged 8 to 12 years during morning recess. This effect study showed that more adjustments to the physical environment of schoolyards, by introducing PA stimuli, led to more time spent in LPA. It seems likely that this change arose from a switch from SB to LPA, although the decrease in time spent in SB was nonsignificant. This finding indicates the effectiveness of the schoolyard adjustments across all schoolyards taking part in the interventions, which means that all intervention activities were pooled, regardless of their content and the context in which they were implemented. We also found that gender, age, and weather conditions were likely to affect PA levels over time. Boys were much more likely to spend less time in SB and more time in LPA and MVPA 12 months after baseline measurements than girls, as were younger children compared to older children, except as regards LPA. Additional sensitivity analyses showed that an increased number of interventions in the physical schoolyard environment led to decreased time spent in SB. Moreover, a trend towards significance $(p<.10)$ was found for the time spent in LPA and MVPA after 12 months. The intensity, mainly operationalized in terms of investments, of the changes implemented in the physical environment strongly predicted increased PA levels and decreased time spent in SB during recess. Moreover, the schools' commitment to the project was found to moderate the relationship between the number of physical environment changes implemented and PA levels. More commitment to the project by schools strengthened the effects physical environment interventions on PA and SB.

\section{Effect evaluation of the Active Living project after 1 year and sustainability of effects after 2 years}

Chapter 8 describes the effectiveness of the Active Living project after 12 months. The Active Living project as a whole was not found to be effective in terms of children's time spent in SB, LPA and MVPA after 12 months. Male gender predicted less time spent in SB and more time in LPA and MVPA after one year, and age was found to negatively influence PA over time. Studying the PA interventions in more detail, however, did indicate changes in the time spent in SB and LPA. Implementing four or more physical environment interventions (PEIS) in the school environment led to an increase in the time spent in LPA after 12 months. This increase only showed a trend towards 
significance, but achieved up to a medium effect size. When we focused on the social environment interventions (SEIs) in the school environment, we found similar results for children attending schools that implemented six or more SEls. Implementing just a few PEls or SEls did not increase the time spent in LPA after 12 months. It could therefore be argued that higher exposure to environmental stimuli, i.e. succeeding intervention activities, is necessary to change longer-term PA (Van Sluijs \& McMinn, 2010). We also tested the assumption of an ecological view, i.e. the interaction between the physical and social environments (Maitland, Stratton, Foster, Braham, \& Rosenberg, 2013). Children attending schools that implemented both sufficient PEIs $(\geq 4)$ and SEIs $(\geq 6)$, i.e. the high PSEls group, engaged in significantly more LPA after 12 months than children attending schools implementing lower numbers of PEIs and SEIs.

After 12 months the effectiveness of the executed interventions on PA and SB was limited and subject to implementation conditions. But, taking into account the schools' potential reach of children, the relatively small effects may have an impact from a public health perspective. In particular, most PA intervention elements, such as providing more portable equipment, were relatively easy to implement which enhances transferability to other schools. A prerequisite for such an impact, however, is sustained effectiveness of the intervention. We therefore tested both the pooled effectiveness of the Active Living project as a whole and the effectiveness of the combined number of PA stimuli (PSEIs) after 24 months, which was 12 months after the Active Living project had ended in schools. Note that only half of our sample was eligible for this assessment, as the other half had transited to secondary school and was lost to follow-up. Schools in which less than five children provided valid PA data during the 24-months assessment were excluded from the analysis. These schools were then also excluded from the 12months assessment, to enhance the comparability of the 12- and 24-months results (Figure 9.1). Consequently, 12-months effectiveness analyses were repeated for 467 of the 520 children (90\%). In the 24-months follow-up, 200 children were eligible for the analyses. Data handling procedures and the analytic approach were similar to the approach used in the effectiveness study (see chapter 8 for a detailed description).

Figure 9.1 shows the relative change in the numbers of minutes spent in SB, LPA and MVPA per weekday. Firstly, changes were compared with those of children attending control schools, and are presented for exposure to the Active Living project as a whole. None of the measures showed significant changes in the numbers of minutes spent in SB, LPA or MVPA after 24 months, which is likely impacted by the reduced power in the multilevel multivariate regression analyses. Purely based on changes in the numbers of minutes spent by children in PA, a small increase in the number of minutes spent sedentary was found after 24 months for children exposed to the Active Living project, while a decrease in LPA was found. No changes in MVPA were found for children exposed to Active Living after 24 months. The relative increase in SB and decrease in LPA declined between 12 and 24 months, whereas time spent in MVPA was equal to scores at baseline after a small decline after 12 months (orange lines in Figure 9.1). 


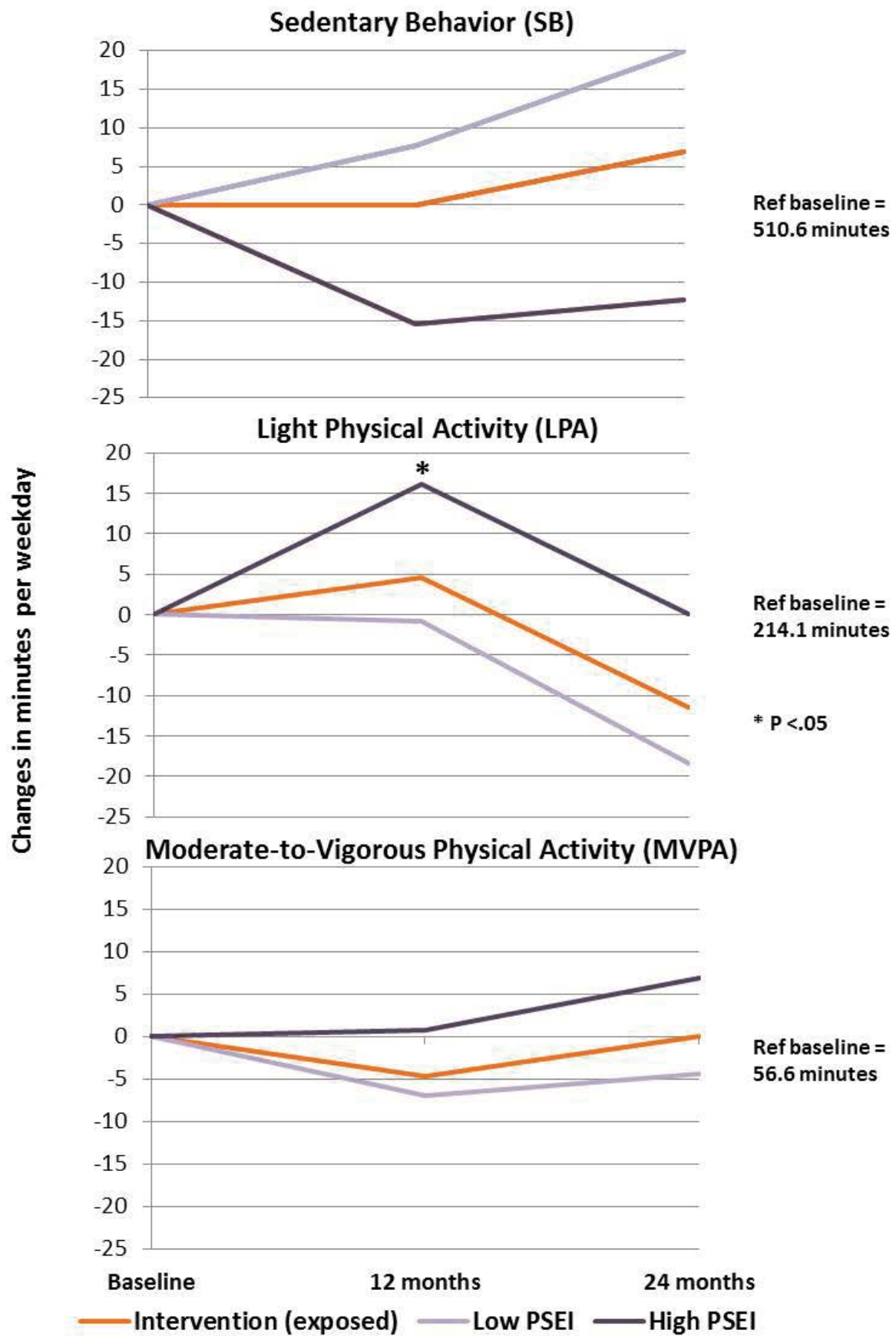

Figure 9.1: 12- and 24-months effectiveness of exposure to the Active Living intervention, for high and low number of combined physical and social environment interventions (PSEIs). 
Secondly, changes are presented for children attending schools in the high- and lowPSEls groups (purple lines in Figure 9.1) and were compared with those for children attending control schools. After 12 months, a positive effect of a high number of combined PSEls was found on decreased time spent in SB. Although non-significant, this result appeared to have been sustained after 24 months. The positive effect on LPA after 12 months found in the high-PSEl group had faded out after 24 months. This fade-out is most likely the result of a small increase in SB and an increase in MVPA (+6.9 minutes per week day) after 24 months. The sustained, though small increase in PA is in line with other studies testing the sustainability of PA intervention effects (Lai et al., 2014).

Children attending schools that implemented only a few physical and/or social environment interventions (low-PSEI group) showed non-significant negative effects on PA outcomes after 12 months. These outcomes were strengthened after 24 months, in that a larger increase in time spent in SB and decrease in time spent in LPA was found, while time spent in MVPA was still slightly decreased after 24 months compared to baseline. The conclusion we drew after 12 months, formulated as 'do it well or don't do it at all', was further corroborated by the results at 24 months and should be taken into account by health promotors and school principals.

\section{METHODOLOGICAL CONSIDERATIONS}

The results of the studies described in this thesis have to be interpreted in light of several methodological considerations. These considerations cover two main topics: (1) study design, population and implementation issues and (2) measurement issues.

\section{Study design, population, and implementation issues}

Several studies (chapters 3, 4, 5, and 6) used a cross-sectional study design, which limits the possibility to draw conclusions about causality (Lindell \& Whitney, 2001; Polit \& Beck, 2004). Insight into the mechanism of PA promotion, i.e. what is working and for whom, and causal relationships between PA and the environment is preferably gained by longitudinal study designs, but these are extremely underrepresented in the literature compared to studies with a cross-sectional design (Atkin, van Sluijs, Dollman, Taylor, \& Stanley, 2016; Larouche, Saunders, Faulkner, Colley, \& Tremblay, 2014). The studies presented in chapters 7 and 8 used a quasi-experimental, longitudinal design, and not only collected longitudinal data but also used environmental manipulations, i.e. interventions, to study their effects on children's PA levels. With respect to the design of the effectiveness study of PA schoolyard interventions on children's PA and SB during morning recess (chapter 7 ), it should be noted that the implemented activities were part of the larger Active Living study (chapter 8). Simultaneously, PA stimulating activities to support PA during leisure time and AST have been implemented in the 
schools' environments which threatened the validity of the chosen study design. Although it is hypothesized that other interventions in the school environment were unlikely to affect the effectiveness of PA schoolyard interventions on children's PA and SB during morning recess, a potential spill-over effect of the additional activities cannot be ruled out completely.

The gold standard for intervention studies is considered to be the randomized controlled trial (RCT), but an RCT may not be feasible for studying the development, implementation, and effectiveness of environmental changes on children's behavior in a real-life setting (such as Active Living) or testing the effectiveness of natural experiments. To meet the end-users' needs, stakeholders need to be involved throughout the various stages of the intervention process. This was also the case in the Active Living project, as we did not want to apply a one-size-fits-all approach. Interventions were designed and created from the input and needs reported by schools, teachers, parents, and children (chapter 2). As a result, the Active Living approach would affect either the randomization or the control aspect of an RCT. Randomization was hardly possible as input by users was necessary. We decided to check the support for the project from the local municipal authorities and the schools, to decide on their inclusion in the intervention condition. In practice, we could have opted for a randomized design, but this carries an increased risk of drop-out and the risk of lengthy processes having to be gone through before being able to start developing and implementing interventions, caused by a potential lack of local support. The 'controlled' criterion for an RCT was not completely fulfilled in our project either. Although all intervention schools were matched to control schools based on several criteria (see chapter 2), for instance local SES scores and degree of urbanization, using a real-life setting cannot rule out any unintended influences during the project period. Besides the matching criteria, we were able to control for differences in seasonal effects which has often been indicated as confounders (Goodman et al., 2014; Oreskovic et al., 2012). Yet, we did not control for PA-related activities control schools possibly performed during the project period. In addition, as the Active Living project focused on interaction between the school environment and the surrounding neighborhood, intervention sites covered about two square kilometers, and there was a chance that unnoticed, temporary environmental changes might take place in these areas. The main goal of the Active Living project, however, was to influence children's PA levels by means of environmental adjustments. Therefore, we valued the advantages of implementing the interventions in a real-life setting more highly than the disadvantages of not fulfilling the criteria for an RCT. We therefore decided that the quasi-experimental design we used, in which intervention schools were matched to control schools, was the best alternative to study the effectiveness of Active Living.

Chapter 3 used cross-sectional observational AST data for children, which were gathered during school visits, as a pilot test for the Active Living project. Other studies described in this thesis (chapters 2 and 4-8) used data derived from the Active Living 
study. In total, 21 schools were recruited to take part in the project (10 as intervention and 11 as control schools). None of the schools dropped out during the study. Nevertheless, differences in the degree of engagement and commitment to the project were noticed between intervention schools, the reasons for which remain unclear. Working groups at each intervention school were led by different regional Public Health Services employees, which could have influenced the level of commitment. Other possible reasons are the composition of the working groups, the involvement of the local municipal authorities, and the existing relations (of trust) between schools, the regional Public Health Services and local authorities. Project commitment may also have been influenced by the decisiveness of the school principals, teachers, and parents involved, as the environmental changes introduced led to a certain feeling of success and increased the enthusiasm to proceed. We observed differences in pro-active decisiveness skills between working groups, which in the positive cases worked as a catalyzer for implementing succeeding intervention elements. Contrary, a lack of decisiveness reduced enthusiasm and the number of interventions implemented.

The recruitment of children by schools was considered to have been successful in both the intervention and control condition, as almost all children filled out the questionnaires, nearly $65 \%$ of the children wore measurement equipment, and the same proportion of parents filled out the parental questionnaires at baseline. Retention rates of both participating children and parents over time were considered acceptable (see Figure 2.4), indicating end-user's project support and providing the opportunity to conduct more reliable effect analyses.

In intervention schools we applied a combined top-down and bottom-up approach. Whereas this approach was assumed to ensure that local support and interventions would fit in with local needs, several challenges and risks were faced. An important risk of this approach was revealed when tasks and employees were reshuffled at the regional Public Health Services. In practice, this resulted in suspension of Active Livingrelated activities between the initial baseline measurement (Fall 2012) and the first follow-up (Spring 2013), finally resulting in adjustment of the study design. Moreover, help from municipal authorities was necessary to obtain support for changes in the physical environment in the neighborhoods around schools. Although this support was granted during recruitment discussions, local policy agendas and funding were subject to change during the project. More binding agreements between program developers, Public Health Services and municipal authorities might overcome these implementation issues in future projects.

\section{Measurement issues}

\section{Physical activity}

There is a growing body of evidence for the importance of using objectively assessed physical activity as an outcome measure. Self-reported PA, mostly assessed by 
questionnaires, greatly overestimates PA levels (Chinapaw, Mokkink, van Poppel, van Mechelen, \& Terwee, 2010), and an objective measure of PA provides more accurate data on the PA levels of children. However, classification of PA levels is arbitrary and age-dependent (De Vries et al., 2009). The studies reported on in this thesis that used PA and SB as outcome variables all used objectively assessed PA data based on accelerometers (chapters 5-8). To classify PA levels and enhance comparability between studies, all these studies applied the same literature-derived age-dependent cut-off values (Evenson et al., 2008). However, Evenson's cut-off values are based on one-axis counts-per-minute, whereas we measured children's PA data using triaxial measurement devices (Actigraph, GT3X+). Hence, there was a loss of information in our data due to a lack of validated, age-dependent triaxial cut-off values.

\section{Contextual information; issues of gathering and processing}

Although accelerometer data provides more accurate estimations of time spent in PA and SB than self-reports, capturing the contextual information about where and under what conditions activity takes place is lacking using accelerometers (Ridgers, Timperio, Cerin, \& Salmon, 2015). Contextual information is required to answer the 'where' question, and can be gathered by questionnaires, observations, and GPS and geographical information system (GIS) data (Atkin et al., 2016; Kelly et al., 2016). This is particularly relevant in studies focusing on environmental interventions as it is necessary to know whether someone is actually exposed to the intervened environment. Behavior-specific contextual information is an important step forward towards gaining more insight into the relationship between environment and PA (Timperio et al., 2015).

Retaining participants during all measurement periods is of particular interest in longitudinal studies (Van Sluijs \& Kriemler, 2016), especially when measurement equipment could potentially be perceived as bothersome. In our study we noticed a reduction of the willingness to wear the measurement equipment over time. Whereas children were very excited about wearing the accelerometers and GPS devices during the baseline measurement, this enthusiasm fell during the study period, due to several constraints mentioned by the children, such as 'hampering activities' and 'not "cool" anymore'. These barriers were mostly mentioned about the GPS device, which was considered to be too large. Moreover, just like Bürgi, Tomatis, Murer, \& de Bruin (2015), we found that children were prohibited from wearing the devices while at a sports club. This non-systematic loss of PA data during sports activities may have influenced our results for all-day PA. The (practical) barriers should be taken into account in future studies using such devices, and we recommend oversampling studies at baseline to prevent power issues at follow-up analyses (e.g. Klinker et al., 2014b).

The first step in processing the objectively assessed location and PA data, i.e. GPS and accelerometer data, is to combine the two types of data. Chapters 6 and 7 report on this process, which was conducted using the personal activity and location 
measurement system (PALMS) developed by the University of California, San Diego. To date, no clear consensus is available on how to proceed with these data. Several other studies used self-developed methods for combining the data, potentially impacting on the validity of the results (e.g. Bürgi \& de Bruin, 2016; Dessing et al., 2016; Dessing et al., 2013; Jones, Coombes, Griffin, \& van Sluijs, 2009; Klinker et al., 2014b; Oreskovic et al., 2012). In recent years important steps have been taken with respect to processing GPS, GIS and accelerometer data and developing methodologies to make these data meaningful (Jankowska et al., 2015; Kerr, Duncan, \& Schipperijn, 2011). Combining the two types of data goes beyond a technical challenge of merging time points, locations, and PA levels. Meaningful use of this innovative technique goes a step further: how can we identify meaningful places and meaningful activities in these places? Depending on the predefined research questions, one could opt for a location-driven or a behavior (PA)-driven approach (e.g. Bürgi et al., 2015; Bürgi, Tomatis, Murer, \& de Bruin, 2016; Klinker et al., 2014a; Klinker et al., 2014b).

Another issue is that no clear criteria for valid data have been developed for combined GPS and accelerometer data. A wide range of criteria have been used, ranging from one hour a day up to more stringent criteria like three days with a minimum of eight hours (Bürgi \& de Bruin, 2016; Dessing et al., 2013; Klinker et al., 2014a), potentially affecting the validity of outcomes.

\section{Neighborhood audits}

The studies discussed in chapters 3-6 used environmental audits to study the association between the physical environment and AST or PA and SB. These environmental audits were conducted using the SPACE checklist (De Vries et al., 2010) or an AST-related equivalent (ESATS; chapter 3). SPACE is a validated checklist based on the Neighborhood Environment Walkability Survey (Saelens et al., 2003), and was adapted to the Dutch environment. Adding functional information to the characteristics observed by the instrument might increase the usability of this observation tool. This qualitative add-on might be helpful in improving the environment-behavior relationship and goes beyond opportunities provided by GIS data. As an example, the study discussed in chapter 6 found a positive association between the presence of green space and SB, which was unexpected in view of previous studies focusing on the effects of green space (e.g. Gubbels et al., 2016). In practice, however, the green space we observed had an aesthetic function, which could explain the association we found, but this type of functional information is currently lacking in the checklist. Combining multiple methods of assessing the environment and its functionality, such as GIS data, neighborhood audits and google earth or street view, could further enhance the understanding and use of the environment for PA purposes. 


\section{Exposure measurement}

In the work reported on in this thesis, we chose to ask the health promotors involved to keep logbooks to measure changes in the physical and social environment. In addition, repeated neighborhood audits were performed to assess environmental characteristics and verify changes in the built environment. To better document physical environment changes over time one could opt for repeated environmental audits in combination with repeated geographical information system (GIS) data screening. Our access to highly-detailed GIS data was limited and not sufficiently up-to-date to apply this procedure in our project. Besides, we failed to study the characteristics of the home environment, consequently not enabling us to detect environmental changes in the home environment. Repeated assessments of the physical home environment would be informative. In terms of feasibility, a questionnaire / self-audit might be the best instrument for measuring these changes in the home environment (e.g. Spurrier, Magarey, Golley, Curnow, \& Sawyer, 2008). Accurately documenting the social environment was found to be challenging. Properly documenting the social environment requires a clear and extensive, repeated document analysis for all stakeholders operating in the study area, e.g. schools, sports clubs and welfare organizations. Further, a clear and extensive process evaluation is recommendable for projects in which no one-size-fits-all approach will be used. Recent studies by Craig et al. (2012) and Moore et al. (2015) provide valuable indications for conducting such a comprehensive process evaluation, including implementation fidelity, dose delivered, dose received and reach of the intervention. In addition, changes in policies and rules at both school and municipal level should be checked for as well as financial resources, which might be considered to affect children's PA and SB.

All interventions in the effectiveness study (chapter 8 ) were pooled as an exposure measure rather than specifying them by conceptual PA domain, i.e. PA during AST, PA during at the schoolyard, or PA during leisure time. This limits the capacity to predict behavior-specific outcomes (Giles-Corti et al., 2005) and limits the possibility to indicate the 'active ingredients' of the interventions, with the exception of the number of PA stimuli (Michie \& Abraham, 2004). On the other hand, our method in which we specify the number of PA stimuli adds to the available knowledge by providing an indication of the intervention magnitude.

\section{Parental behavior}

Parental PA and SB (Eriksson, Nordqvist, \& Rasmussen, 2008; Jago, Fox, Page, Brockman, \& Thompson, 2010) and parental PA practices (Davison et al., 2011; Gattshall et al., 2008; Sleddens et al., 2012) are known to influence children's PA and SB. Parental PA and SB, however, were not assessed optimally in the Active Living project. Although raising the burden for participation, we would advocate to objectively assessing PA and SB in both children and their parents simultaneously in future studies, allowing studying the effect of for instance modeling behavior by the parents and parent-child co-activities. Yet, we 
did assess parental PA practices by validated questionnaires. The response rate for the parental questionnaire was about 50\% during the measurement in the spring of 2014 (T2), which was considered reasonable, but there were discrepancies between the information provided by the parents who returned the questionnaire and that of the children who wore an accelerometer correctly. This led to incomplete matches between the valid PA data by the children and the parental data (about 1 in 3). Including parental PA practices in our Active Living effectiveness analyses as a covariate would have reduced the statistical power substantially, but we feel confident that doing so would have increased the explained variance in the tested models. The lack of this information may have biased the relationship between the environment and children's PA.

\section{PA and SB as outcomes of Active Living; Did we miss something?}

The main outcomes reported in the present thesis were the times spent in PA and SB. Based on the aims of the Active Living study, these outcomes were considered to be the most appropriate. However, one could also opt for multiple other (health) outcomes, such as BMI z-scores, skinfolds, waist circumference, and stamina/condition tests or academic achievements. As our main focus was on increasing PA and decreasing SB at a population level by generally non-sports-oriented PA interventions, we hypothesized that no changes would occur in these other health outcome measures during the limited timespan of the project. Adding these more invasive measurements to the study protocol would have increased the burden of participation. Participants' burden from taking part in a study should be limited by paying close attention to the choice of measurement instruments (Kelly, Fitzsimons, \& Baker, 2016), i.e. what instruments fit in best with the research questions. Potential spill-over influences of the interventions on other correlated health behaviors, such as nutrition or social health behaviors (e.g. conflict behavior and bullying), cannot be ruled out beforehand (Mata et al., 2009). As we did not measure these outcomes adequately, it is possible that potential intervention effects have been missed.

\section{THEORETICAL CONSIDERATIONS}

In the Active Living project we chose to adopt the view as presented in the EnRGframework (Figure 1.1; Kremers et al., 2006). Our focus was particularly on the impact of the environment on PA and SB. Further, we studied moderators affecting the environment - PA relationship to estimate for whom the intervention could potentially work and under what conditions. In addition, the 'environment' was studied more closely, allowing us to test interactions between types of environment to establish whether a synergetic effect occurs when types of environment support or oppose each other.

Moreover, we studied PA more closely, e.g. during different time periods and in different locations. From a theoretical perspective, the pathway from environment to 
PA behavior can also be mediated by socio-cognitive factors (Kremers et al., 2006). By a non-comprehensive operationalization of the dual-process view (Kremers et al., 2006) in the work for this thesis, an accurate interpretation of which pathways are followed as a result of environmental changes is impossible. Testing the mediating pathway though would enhance our understanding of the mechanism by which the environment influences the PA behavior in children (Timperio et al., 2015; Van Sluijs et al., 2011; Van Stralen et al., 2011). Depending on the scope of future studies, comprehensively studying the dual-process view as presented in the EnRG-framework might be considered.

\section{Missing link in ecological frameworks and a prospective view}

Accumulation of environmental influences on children's PA and SB was seen in the effectiveness studies conducted for this thesis (chapters 7 and 8). Implementing multilevel, multicomponent interventions in multiple types of environment seemed to be more effective in terms of increasing children's PA and decreasing their SB than implementing multicomponent interventions in one single type of environment or a single-component intervention in multiple types of environment. These findings are in line with the principles incorporated in ecological models, indicating different interacting environmental levels affecting an individual's behavior (Wachs, 1992). Gubbels et al. (2014) underlined this perspective and advocated testing interactions between multiple environmental levels and various types of environment. Although we agree with the idea of allowing environmental influences to interact, a clear theoretical framework indicating the potential impact of such interactions is lacking to date. In addition, we found that most of the available socioecological models and frameworks focus on generic PA as the main outcome without specifying PA types (e.g. Kremers et al., 2006; Sallis et al., 2006; Welk, 1999), although some models go beyond this generic approach and do focus on specific PA behavior, such as walking or cycling (e.g. Alfonzo, 2005; Pikora et al., 2003; Pont et al., 2011). Atkin et al. (2016) recently advocated limiting the aggregation into 'total' PA as it masks PA during specific episodes or time periods. Specific PA episodes can potentially also be captured more accurately by conceptual PA domains, such as PA at school, which are lacking in currently available models and frameworks.

A specification towards the potential impact of environmental changes is also lacking in the frameworks. In line with Gubbels' (2014) and Atkin's (2016) recommendations, we emphasize incorporating principles of dynamic systems theories into ecological frameworks, such as the EnRG framework (Kremers et al., 2006). In systems theories (Thelen \& Smith, 2006), it is assumed that the impact of interacting elements at different environmental levels and types of environments on children's PA behavior is higher than the impact of elements at a single level and in a single type of environment. This indicates that intervening on multiple environmental levels and types 
of environments has the potential for synergetic effects, i.e. one plus one potentially equals more than two. Integrating dynamic systems theory principles into ecological frameworks would contribute to the understanding of the environment - PA relationship and could potentially better quantify the potential impact of environmental changes.

Another shortcoming of current frameworks is the lack of contextual specificity of environments taken into account in predicting changes in PA and SB. The effectiveness and hence the impact of interventions largely depends on contextual factors. We hypothesize that there is a certain threshold that needs to be reached to catalyze PA change, also referred to as tipping point. In environments featuring elements that come quite close to the tipping point, changes in children's PA levels may occur after even one or a few (minor) environmental changes, whereas the efforts have to be substantially larger in environments that are not close to this point (Thelen, Ulrich, \& Wolff, 1991). Implementing the same intervention(s) in these two hypothesized environments might in the first environment (near the tipping point) lead to changes, whereas the exact same intervention in the second environment (not near the tipping point) may not affect PA at all. Researchers are encouraged to monitor and describe the environmental context in which interventions have been implemented more fully and clearly to identify the PA-supportiveness of the school environment. Increasing the level of contextual specificity of environments into ecological frameworks might help overcome the inconsistent findings regarding children's PA determinants and the effectiveness of PA interventions found to date (Atkin et al., 2016).

\section{RECOMMENDATIONS FOR PRACTICE AND FUTURE RESEARCH}

The studies presented in this thesis, the methodological and theoretical considerations, and the practical experiences and insights gained during the Active Living project, enable us to formulate some recommendations for practice and future research.

\section{Recommendations for practice}

- The position of schools in the process of promoting PA and reducing SB should be taken into account. The primary focus in schools is on educational objectives (Boot, 2011), and tight schedules to meet learning objectives in the regular school curriculum might hamper activities that can be perceived as 'extra', such as stimulating PA. Since schools are evaluated on the basis of outcomes regarding children's cognitive performance, health promotion often is perceived as an additional action, leading to the sense that such preventive initiatives are 'secondary objectives'. However, serious consideration and full commitment by schools, i.e. the principals and teachers, is important for interventions to become successful. Linking 
PA activities to increased cognitive functioning of children (Donnelly et al., 2016) can help integrate PA support in a school's main objectives.

- Taking the gains and needs of schools and teachers into account requires improved skills and knowledge on the part of school-based health promotors. A better understanding of the school setting and the challenges schools have to deal with should be acknowledged and health promotion activities, such as encouraging PA, should deal with these school contextual factors. Moreover, successful leadership of a working group and successful implementation of PA interventions depend on interpersonal skills, which are part of a health promotors' leadership style and (convincing) communication skills (Pucher, 2015).

- Support for the interventions among teachers might also be strengthened by emphasizing teachers' immediate gains, such as less conflict behaviors. This could be regarded as the relative advantage of an intervention (Rogers, 2003). This suggestion is supported by our analyses of additional interviews with school principals in which they reported that the Active Living project led to less bullying at schoolyards and less conflict behaviors. These signals were also reported in another (on-going) project (The Healthy Elementary School of the Future) in the same research area, i.e. the Southern Limburg region of the Netherland (Willeboordse et al., 2016).

- Health promotors working in schools or elsewhere should consider the local context in which PA promotion and SB reduction take place. A one-size-fits-all intervention seems suboptimal and health promotors are strongly encouraged to critically consider for whom and how interventions should be developed and implemented. A collaboration between health promotors and school principals, parents, and children is necessary to apply a bottom-up approach in which local support will be enhanced.

- Health promotors might consider focusing intervention elements on parental behaviors, e.g. family routines, to stimulate walking and cycling to school by primary school children rather than focusing directly on children (chapter 4). Moreover, health promotors are encouraged to critically consider the time and place in which intervention activities will be performed (chapter 5 ).

- At the community level, we recommend facilitating aesthetically pleasant neighborhoods, mostly by keeping them clean (Anthamatten et al., 2011; Harmon et al., 2014; Sugiyama et al., 2009), to stimulate PA and the use of AST.

- We recommend implementing comprehensive PA-supporting and SB-preventing intervention packages (Hills et al., 2015). Ideally, school-based PA promotion and SB reduction should occur simultaneously in multiple settings, such as the school environment, home environment and local community (Bürgi et al., 2015; Klinker et al., 2014b; Klinker et al., 2015; Maitland et al., 2013). Communication with parents and parental involvement should be acknowledged and improved to support interaction between the school and home environments and the decisional process concerning the choice for AST. 
- Based on the findings of the intervention studies described in this thesis, exposure to multiple PA stimuli in different environmental types seems most effective. 'Single shot' events are less likely to lead to sustainable PA changes than exposure to multiple, consecutive interventions (Van Sluijs \& McMinn, 2010), i.e. do it well or don't do it at all. Sustainable PA changes are more likely to be achieved when PA support and SB prevention are addressed multiple times during a school year, and should therefore be integrated in the school's policy. Moreover, since children spend a substantial part of the school day in SB, schools should pursue policies towards reducing SB in particular (Bürgi et al., 2016). In addition, there is a need for policies that extend beyond the school day (Faulkner et al., 2014), e.g. policies for the use of active transportation to and from school. Attention should also be paid to ensuring that policies on paper will be put into practice. For instance, all of the schools in our study had a formal policy of children going outdoors during recess, but in practice several schools chose to keep them inside (chapter 7).

\section{Recommendations for future research}

- There have been few studies examining sustainable effects of interventions on children's PA and SB (Lai et al., 2014). From a public health perspective, it would be helpful if effectiveness trials focused on sustainable changes in children's PA levels rather than on short-term PA and SB outcomes (Van Sluijs \& Kriemler, 2016).

- Current findings on determinants of PA and SB and the effectiveness of interventions targeting these behaviors are highly inconsistent (Atkin et al., 2016). High-quality research designs should be used to improve this area of research (Moore et al., 2015). Longitudinal studies are lacking especially on AST (Larouche et al., 2014) and researchers should conduct longitudinal studies on the relation between physical and social environment and AST. Furthermore, the quality of effectiveness trials could be improved by correcting for confounders in the data, such as weather conditions (Van Sluijs \& Kriemler, 2016).

- Supportive actions in multiple types of environment are assumed to strengthen each other's effectiveness. In contrast, single-environment actions might limit each other's potential effectiveness. Although we have provided evidence for the effectiveness of implementing multiple PA stimuli in the physical and social environments, these findings needs to be statistically confirmed by other studies. Intervening in multiple types of environment and subsequently statistically testing these interactive effects would be helpful for understanding the mechanisms by which a supportive PA environment influences PA and SB. This understanding would be further enhanced by combining multiple settings (e.g. school and home environments) for the purpose of promoting PA and preventing SB (Gittelsohn et al., 2015; Gubbels et al., 2014; Henne, Tandon, Frank, \& Saelens, 2014; Maitland et al., 2013; Pont et al., 2011; Thelen \& Smith, 2006; Van Sluijs et al., 2011). Furthermore, one might consider studying these 
interactions within and between conceptual PA domains to ascertain whether changes in one conceptual PA domain, e.g. PA during AST, might lead to sustained changes in overall daily PA or might lead to compensatory behavior in another conceptual PA domain, e.g. PA during leisure time.

- The effectiveness of trials is subject to multiple contextual factors, limiting the generalizability and transferability of the results. Although challenging, researchers should specify and describe contextual information as clearly as possible and monitor exposure to interventions in order to gain more insight in the effectiveness of school-centered interventions (Atkin et al., 2016; Craig et al., 2012; Durlak \& DuPre, 2008; Moore et al., 2015; Van Sluijs \& Kriemler, 2016). Herewith, the identification whether a school environment is near or far away from the tipping point can be addressed more easily.

- Future studies should further develop and adapt PA behavior-specific frameworks that provide guidance for practice. As a theoretical consideration, we have proposed incorporating principles from systems theories into ecological frameworks and in addition increase the level of contextual specificity of environments in order to indicate to health promotors 'how much' effort, i.e. how many interventions or intervention elements, are necessary to achieve sustained effectiveness of PA interventions.

- Although we were able to establish that a certain number of PA stimuli were needed to change children's PA levels, the effects of specific interventions were not studied in detail in this PhD project, and it remains unclear what intervention elements should preferably be implemented.

- Our study found that boys were generally more physically active than girls. This suggests that moderation tests between environmental interventions and interpersonal characteristics would be useful. Moderation analyses could help predict for whom interventions will be effective, e.g. vulnerable groups such as less active children or children living in low SES areas (Anthamatten et al., 2014; Pawlowski, Andersen, Tjornhoj-Thomsen, Troelsen, \& Schipperijn, 2016).

- Conducting time-specific analyses would increase our understanding of PA behavior and how and when PA and SB could best be intervened on (Ridgers et al., 2015). According to the Activitystat hypothesis, an increase in PA at a certain point in time is likely to be compensated by a decrease in PA at a later time (Ridgers et al., 2015; Rowland, 1998). Researchers should perform time-specific analyses to establish what activities positively influence children's PA levels without negatively influencing their PA levels later on.

- GPS data offer an opportunity to establish the locations where PA and SB take place (Jankowska et al., 2015). Further development of methods to unify the processing of combined accelerometer and GPS data across studies would be valuable for comparisons of results across studies. However, to lower the burden of participation 
and reduce drop-out rates in studies gathering these types of data, smaller and less hindering devices should be developed, validated, and implemented.

\section{GENERAL CONCLUSION}

Studies described in this thesis indicate the potential of changing the physical and social school environments for increasing children's physical activity (PA) and decreasing their sedentary behavior (SB). Simultaneously implementing multiple physical and social environment interventions seems more effective than implementing multiple interventions in one type of environment or limited interventions in multiple types of environment. Hence, the comprehensiveness of multiple PA supportive intervention elements seems an essential condition for a synergetic effect of these types of interventions in terms of promoting PA and reducing SB.

The Active Living project has achieved a sustainable impact on the PA levels of primary school children aged 8-12 years by implementing small-scale, low-budget intervention elements, but only when the positive synergetic principles were taken into account, i.e. sufficient physical and social environment interventions. In contrast, when insufficient PA-supportive actions were undertaken, the PA interventions implemented by Active Living were not successful in increasing PA or decreasing SB among primary school children, or even led to unintended outcomes. The effectiveness of the schoolcentered PA interventions largely depends on the contextual conditions in which they are implemented. For instance, the effectiveness of PA interventions on children's PA levels is moderated by the level of commitment on the part of the schools' staff and the intensity of the intervention. Our findings also allow the conclusion that implementing multiple PA intervention elements in the school environment, though challenging, is necessary and feasible. In addition, we identified several associates of PA and SB and active school transport that can help health promotors to better design, time and target PA-supportive interventions. 


\section{References}



Aarts, M.-J., de Vries, S. I., Van Oers, H. A., \& Schuit, A. J. (2012). Outdoor play among children in relation to neighborhood characteristics: A cross-sectional neighborhood observation study. International Journal of Behavioral Nutrition and Physical Activity, 9, 98.

Ahlport, K., Linnan, L., Vaughn, A., Evenson, K., \& Ward, D. (2008). Barriers to and facilitators of walking and bicycling to school: Formative results from the non-motorized travel study. Health Education \& Behavior, 35(2), 221-244.

Ahrens, W., Pigeot, I., Pohlabeln, H., De Henauw, S., Lissner, L., Molnar, D., . . Siani, A. (2014). Prevalence of overweight and obesity in European children below the age of 10. International Journal of Obesity, 38(S2), S99-S107.

Ajzen, I. (1991). The theory of planned behavior. Organizational Behavior and Human Decision Processes, 50(2), 179-211.

Alfonzo, M. A. (2005). To walk or not to walk? The hierarchy of walking needs. Environment and Behavior, 37(6), 808-836.

Andersen, H. B., Pawlowski, C. S., Scheller, H. B., Troelsen, J., Toftager, M., \& Schipperijn, J. (2015). Activating schoolyards: Study design of a quasi-experimental schoolyard intervention study. BMC Public Health, 15, 523.

Andersen, L., Harro, M., Sardinha, L., Froberg, K., Ekelund, U., Brage, S., \& Anderssen, S. (2006). Physical activity and clustered cardiovascular risk in children: A cross-sectional study (The European Youth Heart Study). Lancet, 368, 299 - 304.

Andersen, L., Riddoch, C., Kriemler, S., \& Hills, A. (2011). Physical activity and cardiovascular risk factors in children. British Journal of Sports Medicine, 45, 871-876.

Anthamatten, P., Brink, L., Lampe, S., Greenwood, E., Kingston, B., \& Nigg, C. (2011). An assessment of schoolyard renovation strategies to encourage children's physical activity. International Journal of Behavioral Nutrition and Physical Activity, 8, 27.

Anthamatten, P., Fiene, E., Kutchman, E., Mainar, M., Brink, L., Browning, R., \& Nigg, C. R. (2014). A microgeographic analysis of physical activity behavior within elementary school grounds. American Journal of Health Promotion, 28(6), 403-412.

Arundell, L., Ridgers, N., Veitch, J., Salmon, J., Hinkley, T., \& Timperio, A. (2013). 5-year changes in afterschool physical activity and sedentary behavior. American Journal of Preventive Medicine, 44, 605-611.

Atkin, A. J., Gorely, T., Biddle, S., Marshall, S. J., \& Cameron, N. (2008). Critical hours: Physical activity and sedentary behavior of adolescents after school. Pediatric Exercise Science, 20(4), 446-456.

Atkin, A. J., van Sluijs, E. M., Dollman, J., Taylor, W. C., \& Stanley, R. M. (2016). Identifying correlates and determinants of physical activity in youth: How can we advance the field? Preventive Medicine, 87, 167-169.

Baggett, C. D., Stevens, J., Catellier, D. J., Evenson, K. R., McMurray, R. G., He, K., \& Treuth, M. S. (2010). Compensation or displacement of physical activity in middle-school girls: The trial of activity for adolescent girls. International Journal of Obesity, 34(7), 1193-1199.

Baquet, G., Ridgers, N. D., Blaes, A., Aucouturier, J., Van Praagh, E., \& Berthoin, S. (2014). Objectively assessed recess physical activity in girls and boys from high and low socioeconomic backgrounds. BMC Public Health, 14, 92.

Bauman, A., Reis, R., Sallis, J., Wells, J., Loos, R., \& Martin, B. (2012). Correlates of physical activity: why are some people physically active and others not? Lancet, 380, 258 - 271.

Beets, M. W., Beighle, A., Erwin, H. E., \& Huberty, J. L. (2009). After-school program impact on physical activity and fitness: A meta-analysis. American Journal of Preventive Medicine, 36(6), 527-537.

Beighle, A., Morgan, C., Le Masurier, G., \& Pangrazi, R. (2006). Children's physical activity during recess and outside of school. Journal of School Health, 76(10), 516-520.

Bekkers, V. J. J. M., De Kool, D., \& Straten, G. F. M. (2012). Educational Governance: Strategie, ontwikkeling en effecten [Educational Governance: Strategy, development and effects] NWO/Beleidsgericht Onderzoek Primair Onderwijs. Rotterdam: Erasmus University.

Bennie, J. A., Chau, J. Y., van der Ploeg, H. P., Stamatakis, E., Do, A., \& Bauman, A. (2013). The prevalence and correlates of sitting in European adults - a comparison of 32 Eurobarometer-participating countries. International Journal of Behavioral Nutrition and Physical Activity, 10, 107. 
Biddle, S. J. H., \& Asare, M. (2011). Physical activity and mental health in children and adolescents: A review of reviews. British Journal of Sports Medicine, 45(11), 886-895.

Biddle, S. J. H., Gorely, T., \& Stensel, D. J. (2004). Health-enhancing physical activity and sedentary behaviour in children and adolescents. Journal of Sports Sciences, 22(8), 679-701.

Blaes, A., Ridgers, N. D., Aucouturier, J., Van Praagh, E., Berthoin, S., \& Baquet, G. (2013). Effects of a playground marking intervention on school recess physical activity in French children. Preventive Medicine, 57(5), 580-584.

Boot, N. M. W. M. (2011). Gezondheidsbevordering en voortgezet onderwijs, verstandshuwelijk of echte liefde? Een onderzoek naar de implementatie van schoolgezondheidsbeleid binnen scholen voor voortgezet onderwijs. Maastricht University, Maastricht.

Brennan, L. K., Brownson, R. C., \& Orleans, C. T. (2014). Childhood obesity policy research and practice: Evidence for policy and environmental Strategies. American Journal of Preventive Medicine, 46(1), e1-e16.

Broekhuizen, K., Scholten, A.-M., \& de Vries, S. (2014). The value of (pre)school playgrounds for children's physical activity level: A systematic review. International Journal of Behavioral Nutrition and Physical Activity, 11, 59.

Bronfenbrenner, U. (1979). The ecology of human development: Experiments by nature and design. Cambridge, MA: Harvard University Press.

Brooke, H., Corder, K., Atkin, A., \& van Sluijs, E. F. (2014). A systematic literature review with meta-analyses of within- and between-day differences in objectively measured physical activity in school-aged children. Sports Medicine, 44(10), 1427-1438.

Brown, W. H., Pfeiffer, K. A., Mclver, K. L., Dowda, M., Addy, C. L., \& Pate, R. R. (2009). Social and environmental factors associated with preschoolers' nonsedentary physical activity. Child Development, 80(1), 45-58.

Brownson, R. C., Hoehner, C. M., Day, K., Forsyth, A., \& Sallis, J. F. (2009). Measuring the built environment for physical activity: State of the science. American Journal of Preventive Medicine, 36(4), S99-S123. e112.

Burghard, M., Knitel, K., van Oost, I., Tremblay, M. S., \& Takken, T. (2016). Is our Youth Cycling to Health? Results From the Netherlands' 2016 Report Card on Physical Activity for Children and Youth. Journal of Physical Activity and Health, $13(11$ suppl 2), S218-S224.

Bürgi, R., \& de Bruin, E. D. (2016). Differences in spatial physical activity patterns between weekdays and weekends in primary school children: A cross-sectional study using accelerometry and global positioning system. Sports, 4(3), 36.

Bürgi, R., Tomatis, L., Murer, K., \& de Bruin, E. D. (2015). Localization of physical activity in primary school children using accelerometry and global positioning system. PLoS One, 10(11), e0142223.

Bürgi, R., Tomatis, L., Murer, K., \& de Bruin, E. D. (2016). Spatial physical activity patterns among primary school children living in neighbourhoods of varying socioeconomic status: A cross-sectional study using accelerometry and Global Positioning System. BMC Public Health, 16, 82.

Calthorpe, P. (1993). The next American metropolis: Ecology, community, and the American dream: Princeton Architectural Press.

Cardon, G., Van Cauwenberghe, E., Labarque, V., Haerens, L., \& De Bourdeaudhuij, I. (2008). The contribution of preschool playground factors in explaining children's physical activity during recess. International Journal of Behavioral Nutrition and Physical Activity, 5, 11

Carlson, J. A., Sallis, J. F., Kerr, J., Conway, T. L., Cain, K., Frank, L. D., \& Saelens, B. E. (2014). Built environment characteristics and parent active transportation are associated with active travel to school in youth age 12-15. British Journal of Sports Medicine, 48(22), 1634-1639.

Carver, A., Timperio, A., \& Crawford, D. (2008a). Neighborhood road environments and physical activity among youth: The CLAN study. Journal of Urban Health, 85(4), 532-544.

Carver, A., Timperio, A., \& Crawford, D. (2008b). Playing it safe: The influence of neighbourhood safety on children's physical activity-A review. Health \& Place, 14(2), 217-227.

Carver, A., Timperio, A., Hesketh, K., \& Crawford, D. (2010). Are safety-related features of the road environment associated with smaller declines in physical activity among youth? Journal of Urban Health, 87(1), 29-43. 
Carver, A., Watson, B., Shaw, B., \& Hillman, M. (2013). A comparison study of children's independent mobility in England and Australia. Children's Geographies, 11(4), 461-475.

CBS. (2014). Statline: Nabijheid voorzieningen: Afstand locatie, wijk- en buurtcijfers 2013. Retrieved from Voorburg/Heerlen: http://statline.cbs.nl/

Chillon, P., Evenson, K., Vaughn, A., \& Ward, D. (2011). A systematic review of interventions for promoting active transportation to school. International Journal of Behavioral Nutrition and Physical Activity, 8, 10.

Chinapaw, M. J., Mokkink, L. B., van Poppel, M. N., van Mechelen, W., \& Terwee, C. B. (2010). Physical activity questionnaires for youth: A systematic review of measurement properties. Sports Medicine, 40(7), 539563.

Choi, L., Liu, Z., Matthews, C. E., \& Buchowski, M. S. (2011). Validation of accelerometer wear and nonwear time classification algorithm. Medicine \& Science in Sports \& Exercise, 43(2), 357-364.

Cohen, D., McKenzie, T., Sehgal, A., Williamson, S., Golinelli, D., \& Lurie, N. (2007). Contribution of public parks to physical activity. American Journal of Public Health, 97, 509-514.

Cohen, J. (1988). Statistical power analysis for the behavioral sciences. New York: Hillsdale, Erlbaum.

Colabianchi, N., Maslow, A., \& Swayampakala, K. (2011). Features and amenities of school playgrounds: A direct observation study of utilization and physical activity levels outside of school time. International Journal of Behavioral Nutrition and Physical Activity, 8, 32.

Coombes, E., Jones, A., Page, A., \& Cooper, A. R. (2014). Is change in environmental supportiveness between primary and secondary school associated with a decline in children's physical activity levels? Health \& Place, 29, 171-178.

Cooper, A. R., Jago, R., Southward, E. F., \& Page, A. S. (2012). Active travel and physical activity across the school transition: The PEACH project. Medicine \& Science in Sports \& Exercise, 44(10), 1890-1897.

Cooper, A. R., Page, A. S., Wheeler, B. W., Hillsdon, M., Griew, P., \& Jago, R. (2010). Patterns of GPS measured time outdoors after school and objective physical activity in English children: The PEACH project. International Journal of Behavioral Nutrition and Physical Activity, 7, 31.

Corder, K., van Sluijs, E. M. F., Ekelund, U., Jones, A. P., \& Griffin, S. J. (2010). Changes in children's physical activity over 12 months: Longitudinal results from the SPEEDY study. Pediatrics, 126(4), e926-e935.

Craggs, C., Corder, K., van Sluijs, E. M. F., \& Griffin, S. J. (2011). Determinants of change in physical activity in children and adolescents: A systematic review. American Journal of Preventive Medicine, 40(6), 645-658.

Craig, P., Cooper, C., Gunnell, D., Haw, S., Lawson, K., Macintyre, S., . . . Sutton, M. (2012). Using natural experiments to evaluate population health interventions: New Medical Research Council guidance. Journal of Epidemiology and Community Health, jech-2011-200375.

D'Haese, S., Van Dyck, D., De Bourdeaudhuij, I., \& Cardon, G. (2013). Effectiveness and feasibility of lowering playground density during recess to promote physical activity and decrease sedentary time at primary school. BMC Public Health, 13, 1154.

Dale, D., Corbin, C. B., \& Dale, K. S. (2000). Restricting opportunities to be active during school time: Do children compensate by increasing physical activity levels after school? Research Quarterly for Exercise and Sport, 71(3), 240-248.

Davidson, Z., Simen-Kapeu, A., \& Veugelers, P. J. (2010). Neighborhood determinants of self-efficacy, physical activity, and body weights among Canadian children. Health \& Place, 16(3), 567-572.

Davison, K. K. (2004). Activity-related support from parents, peers, and siblings and adolescents' physical activity: Are there gender differences? Journal of Physical Activity \& Health, 1, 363-376.

Davison, K. K., Li, K., Baskin, M. L., Cox, T., \& Affuso, O. (2011). Measuring parental support for children's physical activity in white and African American parents: The Activity Support Scale for Multiple Groups (ACTS-MG). Preventive Medicine, 52(1), 39-43

De Vries, S. I., Bakker, I., Van Overbeek, K., Boer, N., Hopman-Rock, M., \& Jeugd, P. (2005). Kinderen in prioriteitswijken: lichamelijke (in)activiteit en overgewicht. (Children in priority neighborhoods: physical (in)activity and overweight.) Leiden: TNO Kwaliteit van Leven.

De Vries, S. I., \& Chorus, A. M. J. (2010). Bewegen in Nederland: jeugdigen van 4-17 jaar (Physical activity in the Netherlands: youngsters between 4-17 years old). In V. H. Hildebrandt, A. M. J. Chorus, \& J. H. Stubbe (Eds.), TNO Trendrapport Bewegen en Gezondheid (pp. pp. 57-76). Leiden: De Bink. 
De Vries, S. I., Hopman-Rock, M., Bakker, I., Hirasing, R. A., \& Van Mechelen, W. (2010). Built environmental correlates of walking and cycling in Dutch urban children: Results from the SPACE study. International Journal of Environmental Research and Public Health, 7(5), 2309-2324.

De Vries, S. I., Hopman-Rock, M., Bakker, I., \& van Mechelen, W. (2009). Meeting the 60-Min physical activity guideline: Effect of operationalization. Medicine \& Science in Sports \& Exercise, 41(1), 81-86.

De Wit, B., Loman, K., Faithfull, K., \& Hinckson, E. A. (2012). Reliability and validity of the hands-up survey in assessing commuting to school in New Zealand elementary school children. Health Promotion Practice, 13(3), 349-354.

Dessing, D., de Vries, S. I., Hegeman, G., Verhagen, E., Van Mechelen, W., \& Pierik, F. H. (2016). Children's route choice during active transportation to school: Difference between shortest and actual route. International Journal of Behavioral Nutrition and Physical Activity, 13, 48.

Dessing, D., Pierik, F., Sterkenburg, R., van, D., Maas, J., \& De Vries, S. (2013). Schoolyard physical activity of 611 year old children assessed by GPS and accelerometry. International Journal of Behavioral Nutrition and Physical Activity, 10, 97.

Ding, D., \& Gebel, K. (2012). Built environment, physical activity, and obesity: What have we learned from reviewing the literature? Health \& Place, 18, 100-105.

Ding, D., Sallis, J. F., Kerr, J., Lee, S., \& Rosenberg, D. E. (2011). Neighborhood environment and physical activity among youth: A review. American Journal of Preventive Medicine, 41(4), 442-455.

Dobbins, M., Husson, H., DeCorby, K., \& LaRocca, R. (2013). School-based physical activity programs for promoting physical activity and fitness in children and adolescents aged 6 to 18. Cochrane Database of Systematic Reviews, 2.

Donnelly, J. E., Hillman, C. H., Castelli, D., Etnier, J. L., Lee, S., Tomporowski, P., . . Szabo-Reed, A. N. (2016). Physical activity, fitness, cognitive function, and academic achievement in children: A systematic review. Medicine \& Science in Sports \& Exercise, 48(6), 1197-1222.

Dössegger, A., Ruch, N., Jimmy, G., Braun-Fahrländer, C., Mäder, U., Hänggi, J., . . Bringolf-Ilser, B. (2014). Reactivity to accelerometer measurement of children and adolescents. Medicine \& Science in Sports \& Exercise, 46(6), 1140-1146.

Dowda, M., McKenzie, T. L., Cohen, D. A., Scott, M. M., Evenson, K. R., Bedimo-Rung, A. L., . . Almeida, M. J. (2007). Commercial venues as supports for physical activity in adolescent girls. Preventive Medicine, 45(2), 163-168.

Durlak, J. A., \& DuPre, E. P. (2008). Implementation matters: A review of research on the influence of implementation on program outcomes and the factors affecting implementation. American Journal of Community Psychology, 41(3-4), 327-350.

Dyment, J. E., Bell, A. C., \& Lucas, A. J. (2009). The relationship between school ground design and intensity of physical activity. Children's Geographies, 7(3), 261-276.

Dyrstad, S. M., Hansen, B. H., Holme, I. M., \& Anderssen, S. A. (2014). Comparison of self-reported versus accelerometer-measured physical activity. Medicine \& Science in Sports \& Exercise, 46(1), 99-106.

Edwardson, C. L., \& Gorely, T. (2010). Parental influences on different types and intensities of physical activity in youth: A systematic review. Psychology of Sport and Exercise, 11(6), 522-535.

Ekelund, U., Luan, J., Sherar, L. B., \& et al. (2012). Moderate to vigorous physical activity and sedentary time and cardiometabolic risk factors in children and adolescents. JAMA, 307(7), 704-712.

Elder, J. P., McGraw, S. A., Stone, E. J., Reed, D. B., Harsha, D. W., Greene, T., \& Wambsgans, K. C. (1994). CATCH: process evaluation of environmental factors and programs. Health Education Quarterly.

Eriksson, M., Nordqvist, T., \& Rasmussen, F. (2008). Associations between parents' and 12-year-old children's sport and vigorous activity: The role of self-esteem and athletic competence. Journal of Physical Activity and Health, 5(3), 359.

Escalante, Y., García-Hermoso, A., Backx, K., \& Saavedra, J. M. (2014). Playground designs to increase physical activity levels during school recess: A systematic review. Health Education \& Behavior, 41(2), 138-144.

Evenson, K., Catellier, D., Gill, K., Ondrak, K., \& McMurray, R. (2008). Calibration of two objective measures of physical activity for children. Journal of Sports Sciences, 26(14), 1557-1565. 
Fairchild, A., \& Mackinnon, D. (2009). A general model for testing mediation and moderation effects. Prevention Science, 10(2), 87-99.

Fairclough, S. J., Beighle, A., Erwin, H., \& Ridgers, N. D. (2012a). School day segmented physical activity patterns of high and low active children. BMC Public Health, 12, 406.

Fairclough, S. J., Ridgers, N. D., \& Welk, G. (2012b). Correlates of children's moderate and vigorous physical activity during weekdays and weekends. Journal of Physical Activity and Health, 9, 129-137.

Faulkner, G., Buliung, R., Flora, P., \& Fusco, C. (2009). Active school transport, physical activity levels and body weight of children and youth: A systematic review. Preventive Medicine, 48(1), 3-8.

Faulkner, G., Richichi, V., Buliung, R., Fusco, C., \& Moola, F. (2010). What's "quickest and easiest?": Parental decision making about school trip mode. International Journal of Behavioral Nutrition and Physical Activity, 7, 62.

Faulkner, G., Zeglen, L., Leatherdale, S., Manske, S., \& Stone, M. (2014). The relationship between school physical activity policy and objectively measured physical activity of elementary school students: a multilevel model analysis. Archives of Public Health, 72(1), 20.

Fedewa, A. L., \& Ahn, S. (2011). The effects of physical activity and physical fitness on children's achievement and cognitive outcomes. Research Quarterly for Exercise and Sport, 82(3), 521-535.

Field, A. (2005). Discovering statistics using SPSS. London, Thousand Oaks, New Delhi: Sage Publications.

Fisher, A., Smith, L., van Jaarsveld, C. H., Sawyer, A., \& Wardle, J. (2015). Are children's activity levels determined by their genes or environment? A systematic review of twin studies. Prev Med Rep, 2, 548553.

Floyd, M. F., Bocarro, J. N., Smith, W. R., Baran, P. K., Moore, R. C., Cosco, N. G., . . . Fang, K. (2011). Parkbased physical activity among children and adolescents. American Journal of Preventive Medicine, 41(3), 258-265.

Frank, L. D., Saelens, B. E., Chapman, J., Sallis, J. F., Kerr, J., Glanz, K., . . . Colburn, T. (2012). Objective assessment of obesogenic environments in youth: geographic information system methods and spatial findings from the Neighborhood Impact on Kids study. American Journal of Preventive Medicine, 42(5), e47-e55.

Gardner, B. (2009). Modelling motivation and habit in stable travel mode contexts. Transportation Research Part F: Traffic Psychology and Behaviour, 12(1), 68-76.

Gardner, B., Bruijn, G.-J., \& Lally, P. (2011). A systematic review and meta-analysis of applications of the selfreport habit index to nutrition and physical activity behaviours. Annals of Behavioral Medicine, 42(2), 174-187.

Gattshall, M. L., Shoup, J. A., Marshall, J. A., Crane, L. A., \& Estabrooks, P. A. (2008). Validation of a survey instrument to assess home environments for physical activity and healthy eating in overweight children. International Journal of Behavioral Nutrition and Physical Activity, 5, 3.

Gidlow, C. J., \& Ellis, N. J. (2011). Neighbourhood green space in deprived urban communities: issues and barriers to use. Local Environment, 16(10), 989-1002.

Giles-Corti, B., Timperio, A., Bull, F., \& Pikora, T. (2005). Understanding physical activity environmental correlates: Increased specificity for ecological models. Exercise and Sport Sciences Reviews, 33(4), 175181.

Gittelsohn, J., Mui, Y., Adam, A., Lin, S., Kharmats, A., Igusa, T., \& Lee, B. (2015). Incorporating systems science principles into the development of obesity prevention interventions: Principles, benefits, and challenges. Current Obesity Reports, 4(2), 174-181.

Gomersall, S., Rowlands, A., English, C., Maher, C., \& Olds, T. (2013). The ActivityStat hypothesis. Sports Medicine, 43(2), 135-149.

Goodman, A., Page, A. S., \& Cooper, A. R. (2014). Daylight saving time as a potential public health intervention: an observational study of evening daylight and objectively-measured physical activity among 23,000 children from 9 countries. International Journal of Behavioral Nutrition and Physical Activity, 11, 84.

Gordon-Larsen, P., Nelson, M. C., Page, P., \& Popkin, B. M. (2006). Inequality in the built environment underlies key health disparities in physical activity and obesity. Pediatrics, 117(2), 417-424. 
Gubbels, J., Van Kann, D., de Vries, N., Thijs, C., \& Kremers, S. (2014). The next step in health behavior research: the need for ecological moderation analyses - an application to diet and physical activity at childcare. International Journal of Behavioral Nutrition and Physical Activity, 11, 52.

Gubbels, J. S., Kremers, S. P., Droomers, M., Hoefnagels, C., Stronks, K., Hosman, C., \& de Vries, S. (2016). The impact of greenery on physical activity and mental health of adolescent and adult residents of deprived neighborhoods: A longitudinal study. Health \& Place, 40, 153-160.

Gubbels, J. S., Kremers, S. P., van Kann, D. H., Stafleu, A., Candel, M. J., Dagnelie, P. C., . . . de Vries, N. K. (2011). Interaction between physical environment, social environment, and child characteristics in determining physical activity at child care. Health Psychology, 30(1), 84-90.

Hager, R. L. (2006). Television viewing and physical activity in children. Journal of Adolescent Health, 39(5), 656-661.

Hallal, P., Andersen, L., Bull, F., Guthold, R., Haskell, W., \& Ekelund, U. (2012). Global physical activity levels: surveillance progress, pitfalls, and prospects. Lancet, 380, 247-257.

Hancox, R. J., Milne, B. J., \& Poulton, R. (2004). Association between child and adolescent television viewing and adult health: A longitudinal birth cohort study. Lancet, 364(9430), 257-262.

Harmon, B. E., Nigg, C. R., Long, C., Amato, K., Anwar, Kutchman, E., . . Hill, J. O. (2014). What matters when children play: Influence of Social Cognitive Theory and perceived environment on levels of physical activity among elementary-aged youth. Psychology of Sport and Exercise, 15(3), 272-279.

Hendriksen, I., Bernaards, C., Hildebrandt, V., \& Hofstetter, H. (2013). Lichamelijke inactiviteit en sedentair gedrag in Nederland 2000-2011. [Physical inactivity and sedentary behavior in the Netherlands 20002011.]. Retrieved from Leiden:

Henne, H. M., Tandon, P. S., Frank, L. D., \& Saelens, B. E. (2014). Parental factors in children's active transport to school. Public Health, 128(7), 643-646.

Herweijer, L., \& Vogels, R. (2004). Ouders over opvoeding en onderwijs. Retrieved from The Hague:

Hildebrandt, V. H., Bernaards, C. M., \& Stubbe, J. H. (2013). Trendrapport Bewegen en Gezondheid 2010/2011 [Trend report Physical Activity and Health 2010/2011]. Retrieved from Leiden:

Hills, A. P., Dengel, D. R., \& Lubans, D. R. (2015). Supporting public health priorities: recommendations for physical education and physical activity promotion in schools. Prog Cardiovasc Dis, 57(4), 368-374.

Hoefer, W. R., McKenzie, T. L., Sallis, J. F., Marshall, S. J., \& Conway, T. L. (2001). Parental provision of transportation for adolescent physical activity. American Journal of Preventive Medicine, 21(1), 48-51.

Hohepa, M., Scragg, R., Schofield, G., Kolt, G. S., \& Schaaf, D. (2007). Social support for youth physical activity: Importance of siblings, parents, friends and school support across a segmented school day. International Journal of Behavioral Nutrition and Physical Activity, 4, 54.

Hubbard, K., Economos, C. D., Bakun, P., Boulos, R., Chui, K., Mueller, M. P., . . Sacheck, J. (2016). Disparities in moderate-to-vigorous physical activity among girls and overweight and obese schoolchildren during school-and out-of-school time. International Journal of Behavioral Nutrition and Physical Activity, 13, 39.

Huberty, J. L., Siahpush, M., Beighle, A., Fuhrmeister, E., Silva, P., \& Welk, G. (2011). Ready for recess: A pilot study to increase physical activity in elementary school children. Journal of School Health, 81(5), 251257.

Hume, C., Timperio, A., Salmon, J., Carver, A., Giles-Corti, B., \& Crawford, D. (2009). Walking and cycling to school: Predictors of increases among children and adolescents. American Journal of Preventive Medicine, 36(3), 195-200.

Hurvitz, P. M., Moudon, A. V., Kang, B., Saelens, B. E., \& Duncan, G. E. (2014). Emerging technologies for assessing physical activity behaviors in space and time. Emerging Technologies to Promote and Evaluate Physical Activity, 8.

Ickes, M. J., Erwin, H., \& Beighle, A. (2013). Systematic review of recess interventions to increase physical activity. Journal of Physical Activity and Health, 10(6), 910-926.

Jago, R., Fox, K. R., Page, A. S., Brockman, R., \& Thompson, J. L. (2010). Parent and child physical activity and sedentary time: Do active parents foster active children? BMC Public Health, 10, 194. 
Jago, R., Sebire, S., Turner, K., Bentley, G., Goodred, J., Fox, K., . . Lucas, P. (2013). Feasibility trial evaluation of a physical activity and screen-viewing course for parents of 6 to 8 year-old children: Teamplay. International Journal of Behavioral Nutrition and Physical Activity, 10, 31.

Jago, R., Wood, L., Sebire, S. J., Edwards, M. J., Davies, B., Banfield, K., . . . Montgomery, A. A. (2014). School travel mode, parenting practices and physical activity among UK Year 5 and 6 children. BMC Public Health, 14, 370.

Jankowska, M., Schipperijn, J., \& Kerr, J. (2015). A framework for using GPS data in physical activity and sedentary behavior studies. Exercise and Sport Sciences Reviews, 43(1), 48-56.

Janssen, I., \& LeBlanc, A. (2010). Systematic review of the health benefits of physical activity and fitness in school-aged children and youth. International Journal of Behavioral Nutrition and Physical Activity, 7, 40.

Janssen, M., Twisk, J. W., Toussaint, H. M., van Mechelen, W., \& Verhagen, E. A. (2015). Effectiveness of the PLAYgrounds programme on PA levels during recess in 6-year-old to 12-year-old children. British Journal of Sports Medicine, 49(4), 259-264.

Jones, A., Coombes, E., Griffin, S., \& van Sluijs, E. (2009). Environmental supportiveness for physical activity in English schoolchildren: A study using Global Positioning Systems. Internatonal Journal of Behavioral Nutrition and Physical Activity, 6, 42

Jones, R. A., Hinkley, T., Okely, A. D., \& Salmon, J. (2013). Tracking physical activity and sedentary behavior in childhood. American Journal of Preventive Medicine, 44(6), 651-658.

Kanters, M. A., Bocarro, J. N., Filardo, M., Edwards, M. B., McKenzie, T. L., \& Floyd, M. F. (2014). Shared use of school facilities with community organizations and afterschool physical activity program participation: A cost-benefit assessment. Journal of School Health, 84(5), 302-309.

Kelly, P., Fitzsimons, C., \& Baker, G. (2016). Should we reframe how we think about physical activity and sedentary behaviour measurement? Validity and reliability reconsidered. International Journal of Behavioral Nutrition and Physical Activity, 13, 32.

Kerr, J., Duncan, S., \& Schipperijn, J. (2011). Using Global Positioning Systems in health research: A practical approach to data collection and processing. American Journal of Preventive Medicine, 41, 532-540.

Kerr, J., Rosenberg, D., Sallis, J., Saelens, B., Frank, L., \& Conway, T. (2006). Active commuting to school: Associations with environment and parental concerns. Medicine \& Science in Sports \& Exercise, 38(4), 787-794.

Kitzman-Ulrich, H., Wilson, D., St. George, S., Lawman, H., Segal, M., \& Fairchild, A. (2010). The integration of a family systems approach for understanding youth obesity, physical activity, and dietary programs. Clinical Child and Family Psychology Review, 13(3), 231-253.

Klinker, C., Schipperijn, J., Christian, H., Kerr, J., Ersboll, A., \& Troelsen, J. (2014a). Using accelerometers and Global Positioning System devices to assess gender and age differences in children's school, transport, leisure and home based physical activity. International Journal of Behavioral Nutrition and Physical Activity, 11, 8.

Klinker, C. D., Schipperijn, J., Kerr, J., Ersbøll, A. K., \& Troelsen, J. (2014b). Context-specific outdoor time and physical activity among school-children across gender and age: Using accelerometers and GPS to advance methods. Frontiers in Public Health, 2, 20.

Klinker, C. D., Schipperijn, J., Toftager, M., Kerr, J., \& Troelsen, J. (2015). When cities move children: Development of a new methodology to assess context-specific physical activity behaviour among children and adolescents using accelerometers and GPS. Health \& Place, 31, 90-99.

Knol, F. (2012). Statusontwikkeling van wijken in Nederland 1998-2010. Sociaal Cultureel Planbureau, Den Haag.

Kohl, H. W., Craig, C. L., Lambert, E. V., Inoue, S., Alkandari, J. R., Leetongin, G., \& Kahlmeier, S. (2012). The pandemic of physical inactivity: Global action for public health. Lancet, 380(9838), 294-305.

Koplan, J. P., Liverman, C. T., \& Kraak, V. I. (2005). Preventing childhood obesity: Health in the balance: Executive summary. Journal of the American Dietetic Association, 105(1), 131-138.

Kremers, S., Dijkman, M., de Meij, J., Jurg, M., \& Brug, J. (2008). Awareness and habit: Important factors in physical activity in children. Health Education, 108(6), 475-488.

Kremers, S. P. (2010). Theory and practice in the study of influences on energy balance-related behaviors. Patient Education and Counseling, 79(3), 291-298. 
Kremers, S. P., \& Brug, J. (2008). Habit strength of physical activity and sedentary behavior among children and adolescents. Pediatric Exercise Science, 20(1), 5.

Kremers, S. P., de Bruijn, G. J., Visscher, T. L., van Mechelen, W., de Vries, N. K., \& Brug, J. (2006). Environmental influences on energy balance-related behaviors: A dual-process view. International Journal of Behavioral Nutrition and Physical Activity, 3, 9.

Kremers, S. P., Visscher, T. L., Seidell, J. C., van Mechelen, W., \& Brug, J. (2005). Cognitive determinants of energy balance-related behaviours: Measurement issues. Sports Medicine, 35(11), 923-933.

Krenn, P. J., Titze, S., Oja, P., Jones, A., \& Ogilvie, D. (2011). Use of Global Positioning Systems to study physical activity and the environment: A systematic review. American Journal of Preventive Medicine, 41(5), 508515.

Kriemler, S., Meyer, U., Martin, E., van Sluijs, E., Andersen, L., \& Martin, B. (2011). Effect of school-based interventions on physical activity and fitness in children and adolescents: A review of reviews and systematic update. British Journal of Sports Medicine, 45, 923-930.

Lai, S. K., Costigan, S. A., Morgan, P. J., Lubans, D. R., Stodden, D. F., Salmon, J., \& Barnett, L. M. (2014). Do school-based interventions focusing on physical activity, fitness, or fundamental movement skill competency produce a sustained impact in these outcomes in children and adolescents? A systematic review of follow-up studies. Sports Medicine, 44(1), 67-79.

Landis, J. R., \& Koch, G. G. (1977). The measurement of observer agreement for categorical data. Biometrics, 33(1), 159-174.

Larouche, R. (2015). Built environment features that promote cycling in school-aged children. Current Obesity Reports, 1-10.

Larouche, R., Saunders, T., Faulkner, G., Colley, R., \& Tremblay, M. (2014). Associations between active school transport and physical actvity, body composition and cardiovascular fitness: A systematic review of 68 studies. Journal of Physical Activity and Health, 11, 206-227.

Lavelle, H. V., Mackay, D. F., \& Pell, J. P. (2012). Systematic review and meta-analysis of school-based interventions to reduce body mass index. Journal of Public Health, 34(3), 360-369.

Lee, A. C., \& Maheswaran, R. (2011). The health benefits of urban green spaces: A review of the evidence. Journal of Public Health, 33(2), 212-222.

Lee, R. E., Booth, K. M., Reese-Smith, J. Y., Regan, G., \& Howard, H. H. (2005). The Physical Activity Resource Assessment (PARA) instrument: Evaluating features, amenities and incivilities of physical activity resources in urban neighborhoods. International Journal of Behavioral Nutrition and Physical Activity, 2, 13.

Leslie, E., Kremer, P., Toumbourou, J. W., \& Williams, J. W. (2010). Gender differences in personal, social and environmental influences on active travel to and from school for Australian adolescents. Journal of Science and Medicine in Sport, 13(6), 597-601.

Lindell, M. K., \& Whitney, D. J. (2001). Accounting for common method variance in cross-sectional research designs. Journal of Applied Psychology, 86(1), 114-121.

Lipsey, M. W. (1990). Design sensitivity: Statistical power for experimental research. Newbury Park, CA: Sage Publication.

Lobstein, T., Baur, L., \& Uauy, R. (2004). Obesity in children and young people: A crisis in public health. Obesity Reviews, 5, 4-85.

Lobstein, T., Jackson-Leach, R., Moodie, M. L., Hall, K. D., Gortmaker, S. L., Swinburn, B. A., . . McPherson, K. (2015). Child and adolescent obesity: Part of a bigger picture. Lancet, 385(9986), 2510-2520.

Lonsdale, C., Rosenkranz, R. R., Peralta, L. R., Bennie, A., Fahey, P., \& Lubans, D. R. (2013). A systematic review and meta-analysis of interventions designed to increase moderate-to-vigorous physical activity in school physical education lessons. Preventive Medicine, 56(2), 152-161.

Loprinzi, P. D., Cardinal, B. J., Loprinzi, K. L., \& Lee, H. (2012). Benefits and environmental determinants of physical activity in children and adolescents. Obesity Facts, 5(4), 597-610.

Lou, D. W. (2014). Sedentary behaviors and youth: Current trends and the impact of health. Retrieved from San Diego, CA:

Loucaides, C. A., Jago, R., \& Charalambous, I. (2009). Promoting physical activity during school break times: Piloting a simple, low cost intervention. Preventive Medicine, 48(4), 332-334. 
Lubans, D. R., Morgan, P. J., Cliff, D. P., Barnett, L. M., \& Okely, A. D. (2010). Fundamental movement skills in children and adolescents: Review of associated health benefits. Sports Medicine, 40(12), 1019-1035.

Maitland, C., Stratton, G., Foster, S., Braham, R., \& Rosenberg, M. (2013). A place for play? The influence of the home physical environment on children's physical activity and sedentary behaviour. International Journal of Behavioral Nutrition and Physical Activity, 10, 99.

Martin, A., Goryakin, Y., \& Suhrcke, M. (2014). Does active commuting improve psychological wellbeing? Longitudinal evidence from eighteen waves of the British household panel survey. Preventive Medicine, 69, 296-303.

Mata, J., Silva, M. N., Vieira, P. N., Carraça, E. V., Andrade, A. M., Coutinho, S. R., . . . Teixeira, P. J. (2009). Motivational "spill-over" during weight control: Increased self-determination and exercise intrinsic motivation predict eating self-regulation. Health Psychology, 28(6), 709.

Mattocks, C., Ness, A., Leary, S., Tilling, K., Blair, S. N., Shield, J., . . . Riddoch, C. J. (2008). Use of accelerometers in a large field-based study of children: Protocols, design issues, and effects on precision Journal of Physical Activity and Health, 5((S1)), S98-S111.

McDonald, N., Brown, A., Marchetti, L., \& Pedroso, M. (2011). U.S. school travel, 2009: An assessment of trends. American Journal of Preventive Medicine, 41(2), 146-151.

McDonald, N. C. (2007). Active transportation to school: Trends among U.S. schoolchildren, 1969-2001. American Journal of Preventive Medicine, 32(6), 509-516.

McDonald, N. C. (2008). Children's mode choice for the school trip: The role of distance and school location in walking to school. Transportation, 35, 23-35.

Mclver, K. L., Brown, W. H., Pfeiffer, K. A., Dowda, M., \& Pate, R. R. (2009). Assessing children's physical activity in their homes: The observational system for recording physical activity in children-home. Journal of Applied Behavior Analysis, 42(1), 1-16.

McKenzie, T., Marshall, S., Sallis, J., \& Conway, T. (2000). Leisure-time physical activity in school environments: An observational study using SOPLAY. Preventive Medicine, 30, 70-77.

McMillan, T. E. (2007). The relative influence of urban form on a child's travel mode to school. Transportation Research Part A: Policy and Practice, 41(1), 69-79.

Metcalf, B., Henley, W., \& Wilkin, T. (2012). Effectiveness of intervention on physical activity of children: Systematic review and meta-analysis of controlled trials with objectively measured outcomes (EarlyBird 54). British Medical Journal, 345, e5888.

Michie, S., \& Abraham, C. (2004). Interventions to change health behaviours: Evidence-based or evidenceinspired? Psychology \& Health, 19(1), 29-49.

Mitchell, J. A., Pate, R. R., Beets, M. W., \& Nader, P. R. (2013). Time spent in sedentary behavior and changes in childhood BMI: A longitudinal study from ages 9 to 15 years. International Journal of Obesity, 37, 54-60.

Moore, G. F., Audrey, S., Barker, M., Bond, L., Bonell, C., Hardeman, W., . . . Wight, D. (2015). Process evaluation of complex interventions: Medical Research Council guidance. British Medical Journal, 350.

Morton, K. L., Corder, K., Suhrcke, M., Harrison, F., Jones, A. P., van Sluijs, E. M., \& Atkin, A. J. (2016). School polices, programmes and facilities, and objectively measured sedentary time, LPA and MVPA: Associations in secondary school and over the transition from primary to secondary school. International Journal of Behavioral Nutrition and Physical Activity, 13, 54.

Mota, J., Santos, P., Guerra, S., Ribeiro, J. C., \& Duarte, J. A. (2003). Patterns of daily physical activity during school days in children and adolescents. American Journal of Human Biology, 15(4), 547-553.

Murtagh, S., Rowe, D., Elliott, M., McMinn, D., \& Nelson, N. (2012). Predicting active school travel: The role of planned behavior and habit strength. International Journal of Behavioral Nutrition and Physical Activity, $9,65$.

Napier, M. A., Brown, B. B., Werner, C. M., \& Gallimore, J. (2011). Walking to school: Community design and child and parent barriers. Journal of Environmental Psychology, 31(1), 45-51.

Nelson, N. M., Foley, E., O'Gorman, D. J., Moyna, N. M., \& Woods, C. B. (2008). Active commuting to school: How far is too far? International Journal of Behavioral Nutrition and Physical Activity, 5, 1.

Nielsen, G., Taylor, R., Williams, S., \& Mann, J. (2010). Permanent play facilities in school playgrounds as a determinant of children's activity. Journal of Physical Activity and Health, 7(4), 490-496. 
Oliver, M., Badland, H., Mavoa, S., Witten, K., Kearns, R., Ellaway, A., . . Schluter, P. J. (2014). Environmental and socio-demographic associates of children's active transport to school: A cross-sectional investigation from the URBAN Study. International Journal of Behavioral Nutrition and Physical Activity, 11, 70.

Ooijendijk, W., Wendel-Vos, W., \& de Vries, S. (2007). Consensus Vragenlijsten Sport en Bewegen [Consensus Questionnaires Sports and Physical Activity]. Retrieved from Leiden

Oreskovic, N., Blossom, J., Field, A., Chiang, S., Winickoff, J., \& Kleinman, R. (2012). Combining Global Positioning System and accelerometer data to determine the locations of physical activity in children. Geospat Health, 6, 263-272.

Ouellette, J., \& Wood, W. (1998). Habit and intention in everyday life: The multiple processes by which past behavior predicts future behavior. Psychology Bulletin, 124, 54-74.

Owen, N., Humpel, N., Leslie, E., Bauman, A., \& Sallis, J. F. (2004). Understanding environmental influences on walking: Review and research agenda. American Journal of Preventive Medicine, 27(1), 67-76.

Pagels, P., Raustorp, A., De Leon, A., Martensson, F., Kylin, M., \& Boldemann, C. (2014). A repeated measurement study investigating the impact of school outdoor environment upon physical activity across ages and seasons in Swedish second, fifth and eighth graders. BMC Public Health, 14, 803.

Panter, J., Jones, A., \& van Sluijs, E. (2008). Environmental determinants of active travel in youth: A review and framework for future research. International Journal of Behavioral Nutrition and Physical Activity, 5, 34.

Parrish, A., Okely, A., Stanley, R., \& Ridgers, N. (2013). The effect of school recess interventions on physical activity: A systematic review. Sports Medicine, 43(4), 287-299.

Parsons, T. J., Power, C., Logan, S., \& Summerbell, C. D. (1999). Childhood predictors of adult obesity: A systematic review. International Journal of Obesity and Related Metabolic Disorders, 23 Supp/ 8, S1-107.

Pate, R., Wang, C., Dowda, M., Farrell, S., \& O'Neill, J. (2006a). Cardiorespiratory fitness levels among US youth 12 to 19 years of age: Findings from the 1999-2002 National Health and Nutrition Examination Survey. Archives of Pediatrics and Adolescent Medicine, 160(10), 1005-1012.

Pate, R. R., Davis, M. G., Robinson, T. N., Stone, E. J., McKenzie, T. L., \& Young, J. C. (2006b). Promoting physical activity in children and youth: A leadership role for schools: A scientific statement from the American Heart Association Council on nutrition, physical activity, and metabolism (Physical Activity Committee) in collaboration with the councils on cardiovascular disease in the young and cardiovascular nursing. Circulation, 114(11), 1214-1224.

Pate, R. R., Trost, S. G., Felton, G. M., Ward, D. S., Dowda, M., \& Saunders, R. (1997). Correlates of physical activity behavior in rural youth. Research Quarterly for Exercise and Sport, 68(3), 241-248.

Pawlowski, C. S., Andersen, H. B., Tjornhoj-Thomsen, T., Troelsen, J., \& Schipperijn, J. (2016). Space, body, time and relationship experiences of recess physical activity: A qualitative case study among the least physical active schoolchildren. BMC Public Health, 16, 16.

Pearson, N., Braithwaite, R. E., Biddle, S. J. H., van Sluijs, E. M. F., \& Atkin, A. J. (2014). Associations between sedentary behaviour and physical activity in children and adolescents: A meta-analysis. Obesity Reviews, 15(8), 666-675.

Pikora, T., Giles-Corti, B., Bull, F., Jamrozik, K., \& Donovan, R. (2003). Developing a framework for assessment of the environmental determinants of walking and cycling. Social Science \& Medicine, 56(8), 1693-1703.

Polit, D. F., \& Beck, C. T. (2004). Nursing research: Principles and methods: Lippincott Williams \& Wilkins.

Pont, K., Ziviani, J., Wadley, D., \& Abbott, R. (2011). The Model of Children's Active Travel (M-CAT): A conceptual framework for examining factors influencing children's active travel. Aust Occup Ther J, 58(3), 138-144.

Pont, K., Ziviani, J., Wadley, D., Bennett, S., \& Abbott, R. (2009). Environmental correlates of children's active transportation: A systematic literature review. Health \& Place, 15(3), 849-862.

Pucher, K. (2015). Optimizing intersectoral collaboration in school health promotion: creating win-win situations and a systematic implementation based on the diagnosis of sustainable collaboration model. Maastricht University, Maastricht.

Reijgersberg, N., Van der Werff, H., \& Lucassen, J. (2013). Nulmeting Bewegingsonderwijs: Onderzoek naar de organisatie van bewegingsonderwijs in het primair onderwijs. Utrecht: Mulier Instituut. 
Remmers, T., Sleddens, E. F., Kremers, S. P., \& Thijs, C. (2015). Moderators of the relationship between physical activity enjoyment and physical activity in children. Journal of Physical Activity and Health, 12(8), 1066-1073.

Rennie, K. L., Johnson, L., \& Jebb, S. A. (2005). Behavioural determinants of obesity. Best Practice \& Research Clinical Endocrinology \& Metabolism, 19(3), 343-358.

Reynolds, K. D., Wolch, J., Byrne, J., Chou, C.-P., Feng, G., Weaver, S., \& Jerrett, M. (2007). Trail characteristics as correlates of urban trail use. American Journal of Health Promotion, 21(4), 335-345.

Rich, C., Griffiths, L., \& Dezateux, C. (2012). Seasonal variation in accelerometer-determined sedentary behaviour and physical activity in children: a review. International Journal of Behavioral Nutrition and Physical Activity, 9(1), 49.

Ridgers, N., Fairclough, S., \& Stratton, G. (2010a). Twelve-month effects of a playground intervention on children's morning and lunchtime recess physical activity levels. Journal of Physical Activity and Health, 7(2), 167-175.

Ridgers, N., Fairclough, S., \& Stratton, G. (2010b). Variables associated with children's physical activity levels during recess: The A-CLASS project. International Journal of Behavioral Nutrition and Physical Activity, 7, 74.

Ridgers, N., Salmon, J., Parrish, A., Stanley, R., \& Okely, A. (2012a). Physical activity during school recess: A systematic review. American Journal of Preventive Medicine, 43(3), 320-328.

Ridgers, N., Stratton, G., \& Fairclough, S. (2006). Physical activity levels of children during school playtime. Sports Medicine, 36(4), 359-371.

Ridgers, N., Stratton, G., Fairclough, S., \& Twisk, J. (2007). Long-term effects of a playground markings and physical structures on children's recess physical activity levels. Preventive Medicine, 44, 393-397.

Ridgers, N. D., Salmon, J., Parrish, A.-M., Stanley, R. M., \& Okely, A. D. (2012b). Physical activity during school recess: A systematic review. American Journal of Preventive Medicine, 43(3), 320-328.

Ridgers, N. D., Timperio, A., Cerin, E., \& Salmon, J. (2015). Within-and between-day associations between children's sitting and physical activity time. BMC Public Health, 15, 950.

Ridgers, N. D., Timperio, A., Crawford, D., \& Salmon, J. (2011). Five-year changes in school recess and lunchtime and the contribution to children's daily physical activity. British Journal of Sports Medicine, 46(10), 741-746.

Rimmer, J. A., \& Rowland, J. L. (2008). Physical activity for youth with disabilities: A critical need in an underserved population. Developmental Neurorehabilitation, 11(2), 141-148.

Roberts, J. D., Ray, R., Biles, A. D., Knight, B., \& Saelens, B. E. (2015). Built environment and active play among Washington DC metropolitan children: A protocol for a cross-sectional study. Archives of Public Health, 73(1), 22.

Rodenburg, G., Oenema, A., Pasma, M., Kremers, S. P. J., \& van de Mheen, D. (2013). Clustering of food and activity preferences in primary school children. Appetite, 60, 123-132.

Rogers, E. M. (2003). Diffusion of innovations. New York: The Free Press.

Rosenberg, D., Ding, D., Sallis, J. F., Kerr, J., Norman, G. J., Durant, N., . . Saelens, B. E. (2009). Neighborhood Environment Walkability Scale for Youth (NEWS-Y): Reliability and relationship with physical activity. Preventive Medicine, 49(2), 213-218.

Rothon, C., Edwards, P., Bhui, K., Viner, R., Taylor, S., \& Stansfeld, S. (2010). Physical activity and depressive symptoms in adolescents: A prospective study. BMC Medicine, 8(1), 32.

Rowland, T. (1998). The biological basis of physical activity. Medicine \& Science in Sports \& Exercise, 30(3), 392-399.

Rush, E., \& Simmons, D. (2014). Physical activity in children: Prevention of obesity and type 2 diabetes In J. H. Goedecke \& E. O. Ojuka (Eds.), Diabetes and Physical Activity (Vol. 60, pp. 113-121). Basel: Med Sport Sci, Karger.

Ryan, R. M., \& Deci, E. L. (2000). Self-determination theory and the facilitation of intrinsic motivation, social development, and well-being. American Psychologist, 55(1), 68.

Saelens, B., Frank, L., Auffrey, C., Whitaker, R., Burdette, H., \& Colabianchi, N. (2006). Measuring physical environments of parks and playgrounds: EAPRS instrument devleopment and inter-rater reliability. Journal of Physical Activity and Health, 3, S190-S207. 
Saelens, B. E., \& Handy, S. L. (2008). Built environment correlates of walking: A review. Medicine \& Science in Sports \& Exercise, 40 (7 Suppl), S550.

Saelens, B. E., Sallis, J. F., Black, J. B., \& Chen, D. (2003). Neighborhood-based differences in physical cctivity: An environment scale evaluation. American Journal of Public Health, 93(9), 1552-1558.

Sallis, J. F. (2009). Measuring physical activity environments: A brief history. American Journal of Preventive Medicine, 36(4), S86-S92.

Sallis, J. F., Alcaraz, J. E., McKenzie, T. L., Hovell, M. F., Kolody, B., \& Nader, P. R. (1992). Parental behavior in relation to physical activity and fitness in 9-year-old children. American Journal of Diseases of Children, 146(11), 1383-1388.

Sallis, J. F., Cervero, R. B., Ascher, W., Henderson, K. A., Kraft, M. K., \& Kerr, J. (2006). An ecological approach to creating active living communities. Annual Review of Public Health, 27, 297-322.

Salmon, J., Salmon, L., Crawford, D., Hume, C., \& Timperio, A. (2007). Associations among individual, social, and environmental barriers and childrens walking or cycling to school. American Journal of Health Promotion, 22(2), 107-113.

Salmon, J., Timperio, A., Telford, A., Carver, A., \& Crawford, D. (2005). Association of family environment with children's television viewing and with low level of physical activity. Obesity Research, 13(11), 1939-1951.

Saunders, T. J., Chaput, J.-P., \& Tremblay, M. S. (2014). Sedentary behaviour as an emerging risk factor for cardiometabolic diseases in children and youth. Canadian Journal of Diabetes, 38(1), 53-61.

Schipperijn, J., Kerr, J., Duncan, S., Madsen, T., Klinker, C. D., \& Troelsen, J. (2014). Dynamic accuracy of GPS receivers for use in health research: A novel method to assess GPS accuracy in real-world settings. Frontiers in Public Health, 2, 21.

Schönbeck, Y., Talma, H., van Dommelen, P., Bakker, B., Buitendijk, S. E., HiraSing, R. A., \& van Buuren, S. (2011). Increase in prevalence of overweight in Dutch children and adolescents: A comparison of nationwide growth studies in 1980, 1997 and 2009. PLoS One, 6(11), e27608.

Scott, M. M., Evenson, K. R., Cohen, D. A., \& Cox, C. E. (2007). Comparing perceived and objectively measured access to recreational facilities as predictors of physical activity in adolescent girls. Journal of Urban Health, 84(3), 346-359.

Sims, J., Scarborough, P., \& Foster, C. (2015). The effectiveness of interventions on sustained childhood physical activity: A systematic review and meta-analysis of controlled studies. PLoS One, 10(7), e0132935.

Singh, A., Uijtdewilligen, L., Twisk, J. R., van Mechelen, W., \& Chinapaw, M. M. (2012). Physical activity and performance at school: A systematic review of the literature including a methodological quality assessment. Archives of Pediatrics \& Adolescent Medicine, 166(1), 49-55.

Singh, A. S., Mulder, C., Twisk, J. W., van Mechelen, W., \& Chinapaw, M. J. (2008). Tracking of childhood overweight into adulthood: A systematic review of the literature. Obesity Reviews, 9(5), 474-488.

Skelton, J. A., Buehler, C., Irby, M. B., \& Grzywacz, J. G. (2012). Where are family theories in family-based obesity treatment?: Conceptualizing the study of families in pediatric weight management. International Journal of Obesity, 36, 891-900.

Sleddens, E. F., Kremers, S. P., De Vries, N. K., \& Thijs, C. (2013). Measuring child temperament: Validation of a 3-item Temperament Measure and 13-item Impulsivity Scale. European Journal of Developmental Psychology, 10(3), 392-401.

Sleddens, E. F., Kremers, S. P., Hughes, S. O., Cross, M. B., Thijs, C., De Vries, N. K., \& O'Connor, T. M. (2012). Physical activity parenting: A systematic review of questionnaires and their associations with child activity levels. Obesity Reviews, 13(11), 1015-1033.

Slingerland, M., \& Borghouts, L. B. (2011). Direct and indirect influence of physical education-based interventions on physical activity: A review. Journal of Physical Activity and Health, 8(6), 866-878.

Smith, L., Norgate, S. H., Cherrett, T., Davies, N., Winstanley, C., \& Harding, M. (2015). Walking school buses as a form of active transportation for children-a review of the evidence. Journal of School Health, 85(3), 197-210.

Sobol-Goldberg, S., Rabinowitz, J., \& Gross, R. (2013). School-based obesity prevention programs: A metaanalysis of randomized controlled trials. Obesity, 21(12), 2422-2428. 
Spence, J. C., \& Lee, R. E. (2003). Toward a comprehensive model of physical activity. Psychology of Sport and Exercise, 4, 7-24.

Spurrier, N. J., Magarey, A. A., Golley, R., Curnow, F., \& Sawyer, M. G. (2008). Relationships between the home environment and physical activity and dietary patterns of preschool children: A cross-sectional study. International Journal of Behavioral Nutrition and Physical Activity, 5, 31.

Stanley, R., Ridley, K., \& Dollman, J. (2012). Correlates of children's time-specific physical activity: A review of the literature. International Journal of Behavioral Nutrition and Physical Activity, 9, 50.

Stewart, O., Moudon, A. V., \& Claybrooke, C. (2014). Multistate evaluation of safe routes to school programs. American Journal of Health Promotion, 28(sp3), S89-S96.

Story, M., Nanney, M., \& Schwartz, M. (2009). Schools and obesity prevention: Creating school environments and policies to promote healthy eating and physical activity. The Milbank Quarterly, 87, 71-100.

Stratton, G., \& Mullan, E. (2005). The effect of multicolor playground markings on children's physical activity level during recess. Preventive Medicine, 41, 828-833.

Sugiyama, T., Leslie, E., Giles-Corti, B., \& Owen, N. (2009). Physical activity for recreation or exercise on neighbourhood streets: Associations with perceived environmental attributes. Health \& Place, 15(4), 1058-1063.

Swinburn, B., Egger, G., \& Raza, F. (1999). Dissecting obesogenic environments: The development and application of a framework for identifying and prioritizing environmental interventions for obesity. Preventive Medicine, 29(6 Pt 1), 563-570.

Taylor, R., Farmer, V., Cameron, S., Meredith-Jones, K., Williams, S., \& Mann, J. (2011). School playgrounds and physical activity policies as predictors of school and home time activity. International Journal of Behavioral Nutrition and Physical Activity, 8, 38.

Telama, R. (2009). Tracking of physical activity from childhood to adulthood: A review. Obesity Facts, 2(3), 187-195.

Thelen, E., \& Smith, L. B. (2006). Dynamic systems theories. In W. Damon \& R. M. Lerner (Eds.), Handbook of Child Psychology (6th edition ed., pp. 258-312). New York: Wiley.

Thelen, E., Ulrich, B. D., \& Wolff, P. H. (1991). Hidden skills: A dynamic systems analysis of treadmill stepping during the first year. Monographs of the society for research in child development, 56(223).

Timperio, A., Ball, K., Salmon, J., Roberts, R., Giles-Corti, B., Simmons, D., . . Crawford, D. (2006). Personal, family, social, and environmental correlates of active commuting to school. American Journal of Preventive Medicine, 30(1), 45-51.

Timperio, A., Crawford, D., Telford, A., \& Salmon, J. (2004). Perceptions about the local neighborhood and walking and cycling among children. Preventive Medicine, 38(1), 39-47.

Timperio, A., Giles-Corti, B., Crawford, D., Andrianopoulos, N., Ball, K., Salmon, J., \& Hume, C. (2008). Features of public open spaces and physical activity among children: Findings from the CLAN study. Preventive Medicine, 47(5), 514-518.

Timperio, A., Reid, J., \& Veitch, J. (2015). Playability: Built and social environment features that promote physical activity within children. Current Obesity Reports, 4(4), 460-476.

Tran, I., Clark, B. R., \& Racette, S. B. (2013). Physical activity during recess outdoors and indoors among urban public school students, St. Louis, Missouri, 2010-2011. Preventing Chronic Disease, 10, E196.

Trapp, G. S. A., Giles-Corti, B., Christian, H. E., Bulsara, M., Timperio, A. F., McCormack, G. R., \& Villaneuva, K. P. (2012). Increasing children's physical activity: Individual, social, and environmental factors associated with walking to and from school. Health Education \& Behavior, 39(2), 172-182.

Tremblay, M., LeBlanc, A., Kho, M., Saunders, T., Larouche, R., Colley, R., . . Gorber, S. (2011). Systematic review of sedentary behaviour and health indicators in school-aged children and youth. International Journal of Behavioral Nutrition and Physical Activity, 8, 98.

Triandis, H. C. (1977). Interpersonal behaviour Monterey, C.A.: Brooke/Cole.

Triandis, H. C. (1980). Values, attitudes, and interpersonal behavior. In H. Howe \& M. Page (Eds.), Nerbraska symposium on motivation 1979 (pp. 195-295). Lincoln, NE: University of Nebraska Press.

Troiano, R. P. (2007). Large-scale applications of accelerometers: New frontiers and new questions. Medicine \& Science in Sports \& Exercise, 39(9), 1501. 
Trost, S., Pate, R., Freedson, P., Sallis, J., \& Taylor, W. (2000). Using objective physical activity measures with youth: How many days of monitoring are needed? Medicine \& Science in Sports \& Exercise, 32, 426-431.

Trost, S. G., Kerr, L. M., Ward, D. S., \& Pate, R. R. (2001). Physical activity and determinants of physical activity in obese and non-obese children. International Journal of Obesity and Related Metabolic Disorders, 25(6), 822-829.

Trudeau, F., \& Shephard, R. (2005). Contribution of school programmes to physical activity levels and attitudes in children and adults. Sports Medicine, 35(2), 89-105.

Trudeau, F., \& Shephard, R. J. (2010). Relationships of physical activity to brain health and the academic performance of schoolchildren. American Journal of Lifestyle Medicine, 4(2), 138-150.

Tucker, P., Irwin, J. D., Gilliland, J., He, M., Larsen, K., \& Hess, P. (2009). Environmental influences on physical activity levels in youth. Health \& Place, 15(1), 357-363.

Tudor-Locke, C., Ainsworth, B. E., \& Popkin, B. M. (2001). Active commuting to school: An overlooked source of childrens physical activity? Sports Medicine, 31(5), 309-313.

Tudor-Locke, C., Lee, S., Morgan, C., Beighle, A., \& Pangrazi, R. (2006). Children's pedometer-determined physical activity during the segmented school day. Medicine \& Science in Sports \& Exercise, 38(10), 17321738.

Uiters, E., \& Verweij, A. (2010). Zijn er verschillen in sociaal-economische status? (Are there differences in social-economic status?) Retrieved from RIVM, Bilthoven: http://www.nationaalkompas.nl/

Van der Ploeg, H., Merom, D., Corpuz, G., \& Bauman, A. (2008). Trends in Australian children traveling to school 1971-2003: Burning petrol or carbohydrates? Preventive Medicine, 46(1), 60-62.

Van Kann, D. H. H., Jansen, M. W. J., De Vries, S. I., De Vries, N. K., \& Kremers, S. P. J. (2015a). Active Living: Development and quasi-experimental evaluation of a school-centered physical activity intervention for primary school children. BMC Public Health, 15, 1315.

Van Kann, D. H. H., Kremers, S. P. J., de Vries, N. K., de Vries, S. I., \& Jansen, M. W. J. (2016). The effect of a school-centered multicomponent intervention on daily physical activity and sedentary behavior in primary school children: The Active Living study. Preventive Medicine, 89, 64-69.

Van Kann, D. H. H., Kremers, S. P. J., Gubbels, J. S., Bartelink, N. H. M., De Vries, S. I., De Vries, N., \& Jansen, M. W. J. (2015b). The association between the physical environment of primary schools and active school transport. Environment and Behavior, 47(4), 418-435.

Van Lenthe, F. J., Brug, J., \& Mackenbach, J. P. (2005). Neighbourhood inequalities in physical inactivity: The role of neighbourhood attractiveness, proximity to local facilities and safety in the Netherlands. Social Science \& Medicine, 60(4), 763-775.

Van Sluijs, E., Fearne, V., Mattocks, C., Riddoch, C., Griffin, S., \& Ness, A. (2009). The contribution of active travel to children's physical activity levels: Cross-sectional results from the ALSPAC study. Preventive Medicine, 48, 519-524.

Van Sluijs, E. M., \& Kriemler, S. (2016). Reflections on physical activity intervention research in young people-dos, don'ts, and critical thoughts. International Journal of Behavioral Nutrition and Physical Activity, 13, 25.

Van Sluijs, E. M., \& McMinn, A. (2010). Preventing obesity in primary schoolchildren. British Medical Journal, 340, c819.

Van Sluijs, E. M., McMinn, A. M., \& Griffin, S. J. (2007). Effectiveness of interventions to promote physical activity in children and adolescents: Systematic review of controlled trials. British Medical Journal, 335(7622), 703.

Van Sluijs, E. M. F., Kriemler, S., \& McMinn, A. M. (2011). The effect of community and family interventions on young people's physical activity levels: A review of reviews and updated systematic review. British Journal of Sports Medicine, 45(11), 914-922.

Van Stralen, M. M., Yildirim, M., te Velde, S. J., Brug, J., van Mechelen, W., \& Chinapaw, M. J. (2011). What works in school-based energy balance behaviour interventions and what does not? A systematic review of mediating mechanisms. International Journal of Obesity, 35, 1251-1265.

Vanwolleghem, G., Van Dyck, D., De Meester, F., De Bourdeaudhuij, I., Cardon, G., \& Gheysen, F. (2016). Which socio-ecological factors associate with a switch to or maintenance of active and passive transport during the transition from primary to secondary school? PLoS One, 11(5), e0156531. 
Veitch, J., Salmon, J., \& Ball, K. (2008). Children's active free play in local neighborhoods: A behavioral mapping study. Health Education Research, 23(5), 870-879.

Veitch, J., Timperio, A., Crawford, D., Abbott, G., Giles-Corti, B., \& Salmon, J. (2011). Is the neighbourhood environment associated with sedentary behaviour outside of school hours among children? Annals of Behavioral Medicine, 41(3), 333-341.

Verloigne, M., Van Lippevelde, W., Maes, L., Yıldırım, M., Chinapaw, M., Manios, Y., . . Brug, J. (2012). Levels of physical activity and sedentary time among 10-to 12-year-old boys and girls across 5 European countries using accelerometers: an observational study within the ENERGY-project. International Journal of Behavioral Nutrition and Physical Activity, 9, 34.

Verrotti, A., Penta, L., Zenzeri, L., Agostinelli, S., \& De Feo, P. (2014). Childhood obesity: prevention and strategies of intervention. A systematic review of school-based interventions in primary schools. Journal of endocrinological investigation, 37(12), 1155-1164.

Verstraete, S. J., Cardon, G. M., De Clercq, D. L., \& De Bourdeaudhuij, I. M. (2006). Increasing children's physical activity levels during recess periods in elementary schools: the effects of providing game equipment. European Journal of Public Health, 16(4), 415-419.

Wachs, T. D. (1992). The nature of nurture. Newbury Park, CA: Sage.

Wang, Y., Cai, L., Wu, Y., Wilson, R. F., Weston, C., Fawole, O., . . Segal, J. (2015). What childhood obesity prevention programmes work? A systematic review and meta-analysis. Obesity Reviews, 16(7), 547-565.

Wang, Y., \& Lim, H. (2012). The global childhood obesity epidemic and the association between socioeconomic status and childhood obesity. International Review of Psychiatry, 24(3), 176-188.

Weir, L. A., Etelson, D., \& Brand, D. A. (2006). Parents' perceptions of neighborhood safety and children's physical activity. Preventive Medicine, 43(3), 212-217.

Welk, G. J. (1999). The youth physical activity promotion model: A conceptual bridge between theory and practice. Quest, 51(1), 5-23.

WHO. (2010). Global recommendations on physical activity for health. Retrieved from World Health Organization, Geneva: www.who.int

Willeboordse, M., Jansen, M., van den Heijkant, S., Simons, A., Winkens, B., de Groot, R., . . Savelberg, H. (2016). The healthy primary school of the future: Study protocol of a quasi-experimental study. BMC Public Health, 16, 639.

Willenberg, L. J., Ashbolt, R., Holland, D., Gibbs, L., MacDougall, C., Garrard, J., . . . Waters, E. (2010). Increasing school playground physical activity: A mixed methods study combining environmental measures and children's perspectives. Journal of Science and Medicine in Sport, 13(2), 210-216.

Williams, A., Henley, W., Williams, C., Hurst, A., Logan, S., \& Wyatt, K. (2013). Systematic review and metaanalysis of the association between childhood overweight and obesity and primary school diet and physical activity policies. International Journal of Behavioral Nutrition and Physical Activity, 10, 101.

Wong, B. Y.-M., Faulkner, G., \& Buliung, R. (2011). GIS measured environmental correlates of active school transport: A systematic review of 14 studies. International Journal of Behavioral Nutrition and Physical Activity, 8, 39.

Zhu, X., \& Lee, C. (2008). Walkability and safety around elementary schools economic and ethnic disparities. American Journal of Preventive Medicine, 34(4), 282-290. 



\section{Samenvatting}



Een gebrek aan fysieke activiteit en een toename aan sedentair gedrag zijn wereldwijd en ook in Nederland groeiende problemen bij kinderen. Onvoldoende fysieke activiteit en teveel sedentair gedrag hebben een relatie met een breed scala aan gezondheidsproblemen, maar worden ook in relatie gebracht met mentale en cognitieve problemen. Deze problemen hebben een directe invloed op het welbevinden van kinderen en de ziektelast die deze kinderen dragen, zowel op korte termijn, maar zeker tijdens latere fases in het leven. In het kader van gezondheidsbevordering verdient het stimuleren van fysieke activiteit en het verminderen van sedentair gedrag daarom hoge prioriteit.

De studies beschreven in dit proefschrift hebben betrekking op de relatie die de basisschoolomgeving heeft op de hoeveelheid fysieke activiteit en sedentair gedrag van kinderen in de leeftijd 8 tot 12 jaar. Een aantal van deze studies beschrijft welk effect aanpassingen in de fysieke en sociale omgeving van basisscholen hebben op de fysieke activiteit en het sedentair gedrag van basisschoolleerlingen. Deze aanpassingen zijn ontwikkeld, geïmplementeerd en geëvalueerd in het kader van het Active Living project. Het hoofddoel van dit proefschrift was de invloed van de schoolomgeving op de hoeveelheid beweging van kinderen te bestuderen en in het bijzonder indicaties en aanbevelingen te geven hoe aanpassingen in deze schoolomgeving kunnen bijdragen aan meer fysieke activiteit en een vermindering van sedentair gedrag van kinderen in deze leeftijdscategorie.

In hoofdstuk 1 wordt een uiteenzetting gegeven over de gevolgen van fysieke inactiviteit en teveel sedentair gedrag, mogelijke determinanten van deze gedragingen en hoe deze determinanten, en dan omgevingsdeterminanten in het bijzonder, gerelateerd zijn aan fysieke activiteit en sedentair gedrag. Daarnaast wordt een beknopt overzicht gegeven van bestaande empirie op het gebied van beweegstimulering op school en welke omgevingsaanpassingen in de schoolomgeving een invloed kunnen hebben op het beweeggedrag van basisschoolleerlingen. Vervolgens wordt in dit hoofdstuk de rationale benoemd voor de uitvoering van een beweegstimuleringsinterventie, het Active Living project, op basisscholen in Zuid Limburg in Nederland. Tot slot beschrijft dit hoofdstuk de doelstellingen van Active Living en de onderzoeksvragen die in dit proefschrift zullen worden beantwoord.

Hoofdstuk 2 beschrijft de ontwikkeling, implementatie en het onderzoeksdesign van het onderzoek naar de effecten van Active Living. Active Living is geïntroduceerd als een school-gecentreerde aanpak die zich richtte op het stimuleren van beweging en het verminderen van sedentair gedrag. De term school-gecentreerd refereert naar enerzijds de maatschappelijk centrale functie die een school vervult in het leven van kinderen en daarnaast naar de geografisch centrale ligging die een basisschool heeft in de wijk in Nederland. De effectevaluatie van Active Living werd uitgevoerd middels een quasiexperimenteel onderzoeksdesign. Aan Active Living namen 21 scholen deel gedurende de twee schooljaren (2012-2014), met 10 scholen als interventiegroep en 11 scholen als controlegroep. In totaal namen meer dan 1300 8-12 jarige kinderen deel aan het 
onderzoek, waarvan ongeveer 800 kinderen een beweegmeter hebben gedragen tijdens 3 geplande meetmomenten. Daarnaast heeft een deel van deze kinderen ook een GPS meter gedragen. De focus van Active Living lag op: (1) het stimuleren van actief transport naar school, (2) beweegstimulering op school en dan met name op het schoolplein en (3) beweegstimulering in de vrije tijd. Voor de ontwikkeling van beweegstimuleringsinterventies binnen Active Living interventiescholen werd gebruik gemaakt van een gecombineerde bottom-up en top-down benadering. Deze benadering hield in dat iedere interventieschool een werkgroep vormde waarin een vertegenwoordiging van school (directie en docenten), ouders en gemeentefunctionarissen, onder leiding van een voorzitter van de GGD Zuid Limburg eigen beweegprioriteiten stelden voor de specifieke school. De werkgroepen beschikten over autonomie om eigen prioriteiten te stellen en afhankelijk hiervan ontwikkelden en voerden zij op maat interventies uit (bottom-up). De ontwikkelde en uitgevoerde interventies moesten passen binnen de focus van Active Living. Een wetenschappelijk begeleidingsteam was verantwoordelijke voor de 'toetsing' van de geschiktheid van de beweeginterventies. Tevens voedde dit team de werkgroepen van evidence-based ideeën om op de gestelde prioriteiten te kunnen interveniëren (top-down). De toegepaste benadering bleek in de praktijk succesvol om draagvlak te creëren en op maat interventieontwikkeling en uitvoer te faciliteren. Daarmee lijkt deze benaderingswijze aan te raden voor toekomstige projecten.

Hoofdstuk 3 presenteert de resultaten van een studie naar welke fysieke omgevingskarakteristieken geassocieerd zijn met het gebruik van actief transport naar school. Twee op de drie kinderen kwamen met actief transport naar school, waarbij een toename zichtbaar was naarmate kinderen ouder werden. De fysieke omgevingskarakteristieken werden geobserveerd aan de hand van een gevalideerde checklist en werden op basis van theorie samengevoegd in een viertal omgevingsclusters: bestemming, veiligheid, esthetiek en functionaliteit. Voor jonge kinderen (5-9 jaar; groep 1 tot en met 5) was geen van de fysieke omgevingsclusters geassocieerd met actief transport naar school. Voor oudere kinderen (9-12 jaar; groep 6 tot en met 8) daarentegen, was de esthetiek om de schoolomgeving positief geassocieerd met het gebruik van actief transport naar school. De veiligheid in de schoolomgeving leek hierbij een voorwaardelijkheid te vormen, oftewel zodra een schoolomgeving voldoende veilig is, wordt de esthetiek belangrijk in het verklaren van transportgedrag naar school. Een goede staat van onderhoud van lokale groenvoorzieningen en de afwezigheid van straatvuil bleken de sterkst voorspellende esthetische omgevingskarakteristieken binnen het esthetiek cluster. Gemeentes kunnen door voldoende aandacht te geven aan de esthetische aspecten in de schoolomgeving een bijdrage leveren aan het gebruik van actief transport naar school.

De studie die gepresenteerd wordt in hoofdstuk 4 focust eveneens op de associatie tussen fysieke omgevingskarakteristieken van basisscholen en actief transport naar school van leerlingen in de leeftijd van 8 tot en met 12 jaar. Deze studie had bijzondere 
aandacht voor routines met betrekking tot het gebruik van actief transport door ouders (PATRns). Deze PATRns waren zowel direct geassocieerd met het gebruik van actief transport naar school door kinderen en ze bleken ook een moderator te zijn van de relatie tussen omgevingsfactoren en actief transport naar school. Afstand tot school was de belangrijkste voorspeller van de modus van transport, waarbij een grotere afstand tot school de kans verkleinde dat een kind actief transport naar school gebruikte. PATRns hadden echter een modererende werking op deze associatie. Wanneer kinderen opgroeien in gezinnen waar lopen en fietsen min of meer routinematig plaatsvindt, verkleint de barrière die afstand opwerpt. Omgekeerd, voor kinderen die opgroeien in gezinnen waar lopen en fietsen niet routinematig plaatsvindt, lijken de barrières te worden vergroot. Deze bevinding impliceert dat het stimuleren van actief transport naar school door kinderen kan plaatsvinden door lopen en fietsen door ouders in alle contexten (dus niet alleen naar school) te stimuleren.

Hoofdstuk 5 beschrijft een studie waarin het objectief gemeten beweeggedrag van kinderen werd gerelateerd aan de bespeelbaarheid (playability) van wijk in een straal van 800 meter rondom basisscholen. In deze studie werd gevonden dat de bespeelbaarheid van de buurt rondom scholen was geassocieerd met de hoeveelheid lichte en matig-tot-zware fysieke activiteit die kinderen na school uitvoeren. Ongeveer $30 \%$ van de fysieke activiteit van kinderen buiten school werd door bespeelbaarheid verklaard. Daarnaast werd gevonden dat de associatie het sterkst was voor kinderen die binnen 800 meter van school wonen. Tijd specifieke analyses toonden aan dat deze associatie het sterkst tot uiting kwam in de periode direct na school tot $18 \mathrm{u}$ in de avond. Deze uitkomsten geven enerzijds indicaties dat een bespeelbare schoolomgeving bijdraagt aan fysieke activiteit en geeft daarnaast aanknopingspunten voor uitvoerders van beweeginitiatieven waar en wanneer zij hun activiteiten het best kunnen laten plaatsvinden.

De studie die in hoofdstuk 6 wordt beschreven, onderzocht de associatie tussen fysieke, sociale en beleidsmatige karakteristieken van het schoolplein en de hoeveelheid sedentair gedrag en matig-tot-zware fysieke activiteit op het schoolplein voor, tijdens en na schooltijd. Hierbij werd gebruik gemaakt van een innovatieve koppeling tussen objectieve beweegdata (accelerometrie) en GPS data. Deze datakoppeling maakte het mogelijk om het beweeggedrag van kinderen op het schoolplein te relateren aan fysieke, sociale en beleidsmatige karakteristieken van het schoolplein. Dagelijks brachten kinderen 54 minuten op het schoolplein door waarvan 9 minuten matig-totzwaar fysiek actief en 20 minuten sedentair. Jongens bleken actiever op het schoolplein dan meisjes. Vaste speeltoestellen, zoals voetbal doeltjes en rekstokken, de betrokkenheid van docenten bij het initiëren van beweegactiviteiten en beleid waar wel en niet gespeeld mag worden met een bal, waren geassocieerd met meer matig-totzware fysieke activiteit en minder sedentair gedrag. Scholen kunnen bijdragen aan beweegstimulering en een vermindering van sedentair gedrag op het schoolplein door 
kritisch te kijken naar de fysieke en sociale schoolpleinomgeving alsmede door een ondersteunend beleid ten aanzien van fysieke activiteit op het schoolplein te voeren.

Hoofdstuk 7 geeft de resultaten weer van een longitudinale studie naar de effectiviteit van fysieke en sociale schoolpleininterventies op de hoeveelheid sedentair gedrag en lichte en matig-tot-zware fysieke activiteit van 8-11 jarige basisschoolleerlingen tijdens pauzes na 12 maanden. Het activiteitenniveau van kinderen werd objectief bepaald en een subgroep droeg eveneens een GPS meter. Aan de hand van GPS analyses werd vastgesteld dat bij 3 van 10 schoolparen de kinderen van tenminste één school van de gepaarde scholen (interventie of controle school) tijdens de pauze niet naar het schoolplein zijn geweest tijdens de ochtendpauze. Deze schoolparen werden uitgesloten waardoor er uiteindelijk 376 kinderen werden geanalyseerd in deze studie. Een klein positief effect werd gevonden voor de tijd die werd doorgebracht in lichte fysieke activiteit (+5.9\%), grotendeels als verschuiving van de tijd die sedentair werd doorgebracht (-5.4\%). Meer fysieke veranderingen op het schoolplein waren de belangrijkste voorspeller van deze verschuiving. De effectiviteit werd echter gemodereerd door de omvang van de interventies en de betrokkenheid van scholen bij het project. Interventies van grotere omvang en meer betrokkenheid van scholen bij het project leidden tot positievere resultaten. Voor matig-tot-zware fysieke activiteit werden geen effecten gevonden. Op basis van resultaten beschreven in deze studie kan worden aanbevolen dat meer fysieke veranderingen op het schoolplein wenselijk zijn, wat een voortdurende aandacht voor beweegstimulering op schoolplein vergt om duurzame verandering in beweeg- en sedentair gedrag teweeg te brengen.

Hoofdstuk 8 beschrijft de effectiviteit van de Active Living aanpak op dagelijks sedentair gedrag, lichte activiteit en matig-tot-zware activiteit van 8-12 jarige kinderen na 12 maanden. Deze resultaten werden beschreven op basis van objectieve beweegdata van 520 kinderen verdeeld over 18 scholen ( 9 interventie en 9 controle scholen). Op basis van een eenvoudige maat die weergeeft om iemand wel of niet is blootgesteld aan Active Living kan worden geconcludeerd dat er geen effect op fysieke activiteit of sedentair gedrag waarneembaar is. Het feit dat Active Living een op maat aanpak betrof leidde tot een grote diversiteit aan interventies op scholen. Deze diversiteit heeft geleid tot analyses die meer recht doen aan deze verschillende pakketten aan interventies per school. Hiertoe zijn analyses uitgevoerd waarin het aantal fysieke en sociale interventies zijn meegenomen als onafhankelijke variabelen. Hieruit bleek dat kinderen die op scholen zaten waar zowel voldoende ( $N \geq 4$ ) fysieke als ook sociale $(\mathrm{N} \geq 6)$ werden geïmplementeerd gemiddeld ruim 15 minuten per dag minder sedentair gedrag vertoonden na 12 maanden. Dit leidde tot een toename van 15 minuten die licht actief werden doorgebracht met een medium effect size ( $E S=0.41$ ). De Active Living benadering heeft derhalve de potentie om kinderen te activeren, echter dient het aantal beweegstimuli in zowel de fysieke als de sociale omgeving van school substantieel te zijn om dit effect te bereiken na 12 maanden. Een onvoldoende groot aantal veranderingen in de fysieke en sociale omgeving van school leidde niet tot 
een toename aan fysieke activiteit of een vermindering van sedentair gedrag tijdens schooldagen. Op basis hiervan werd geconcludeerd: 'doe het goed of doe het niet'.

Tot slot worden in hoofdstuk 9 van dit proefschrift de belangrijkste bevindingen uit de uitgevoerde studies bediscussieerd en in een breder perspectief geplaatst. Tevens worden in dit hoofdstuk resultaten van een additionele meting 1 jaar na afloop van Active Living (2015) gepresenteerd. Daarnaast worden op basis van de uitgevoerde studies aanbevelingen voor toekomstig onderzoek en voor praktijkprofessionals geformuleerd om beweegstimulering te bevorderen en sedentair gedrag te verminderen. De belangrijkste conclusie die uit de studies gepresenteerd in dit proefschrift kan worden getrokken is dat duurzame (tot 2 jaar na implementatie) beweegstimulering en reductie van sedentair gedrag op schooldagen mogelijk is door aanpassingen in de fysieke en sociale omgeving van basisscholen, mits deze aanpassingen voldoen aan bepaalde voorwaardes in termen van hoeveelheid.

Een gecombineerde bottom-up en top-down benadering lijkt een geschikte werkwijze om draagvlak te krijgen voor deze duurzame aanpassingen in de fysieke en sociale schoolomgeving. Daarnaast kan worden geconcludeerd dat een synergetisch effect kan worden bereikt wanneer aanpassingen in zowel de fysieke en sociale omgeving worden doorgevoerd. Oftewel, het is aan te raden veranderingen in de fysieke omgeving van scholen door te voeren, maar tegelijkertijd dient ook het gebruik van deze fysieke omgeving te worden gestimuleerd (sociale omgeving) om het grootste effect te bereiken. Hiermee lijkt de conclusie 'doe het goed of doe het niet' gerechtvaardigd bij de werkwijze en veranderingen zoals uitgevoerd binnen Active Living. 



\section{Summary}



Decreasing levels of physical activity (PA) and increasing levels of sedentary behavior (SB) are growing problems, globally as well as in the Netherlands. A lack of PA is associated with numerous health problems, impaired cognitive performance and decreased mental well-being. These disorders can have an immediate influence on children's well-being and health, but also influence well-being and health during later stages in their lives. The promotion of physical activity (PA) and the reduction of sedentary behaviors (SB) should therefore be prioritized in the context of health promotion.

Studies reported on in this thesis focused on the relationship between the primary school environment and the PA levels of children aged 8-12 years. A few of these studies focused on the effect of adjustments to the physical and social environment of primary school on children's PA and SB. These adjustments were developed, implemented, and evaluated as part of the Active Living project. The main goal of the research presented in this thesis was to examine the influence of the school environment on children's PA levels and in particular to provide suggestions and recommendations regarding the way environmental changes can affect the PA and SB of children in this age range.

In chapter 1 the risks of being physically inactive are explained and the determinants of PA and SB are introduced. In addition, the relationship between these determinants and $\mathrm{PA}$ and $\mathrm{SB}$ is clarified, focusing particularly on the environmental determinants. A brief outline of the available empirical evidence about school-based PA promotion is presented, focusing on the evidence regarding the effects of changes to the school environment on PA levels of primary school children. Subsequently, the chapter states the rationale for carrying out a PA intervention, the Active Living Project, at primary schools in the Southern Limburg region of the Netherlands. Finally, the chapter describes the goals of Active Living and the research questions that were addressed by the studies described in this thesis.

Chapter 2 describes the development and implementation of Active Living and the research designed to evaluate the program's effectiveness. Active Living is introduced as a school-centered multicomponent intervention to promote PA and reduce SB. The term school-centered refers both to the central social position schools take up in children's lives and to the geographically central position primary schools have within neighborhoods in the Netherlands.

The study examining the effectiveness of Active Living used a quasi-experimental design. Twenty-one primary schools took part in the Active Living project, which ran for two school years (2012-2014). These schools included 10 intervention schools and 11 control schools. In total, over 1300 children aged 8-12 years were included in the study, about 800 of whom wore an accelerometer during three scheduled measurements. In addition, a subsample of these children wore a GPS device. Active Living focused on: (1) increasing active school transportation (AST), (2) increasing PA in school and in particular at the schoolyard, and (3) increasing PA in leisure time. The PA interventions in the schools were developed using a combined bottom-up and top-down approach. 
This approach involved forming a working group at every intervention school, led by an employee of the Southern Limburg Public Health Services. Other members of the working groups included representatives of the school in question (principal and teachers), representatives of parents, and local municipal officers. The autonomous working groups prioritized their own set of goals to intervene on, in line with the principles of the Active Living project (bottom-up). Depending on these priorities, tailored PA intervention were developed and implemented. A scientific advisory group tested the suitability of the proposed PA intervention for each school. This scientific advisory group also informed the working group about evidence-based methods to intervene on their prioritized goals (top-down). This approach proved to be successful in practice to create local support and to facilitate tailored PA intervention development and implementation. This approach would thus also be suitable for future projects.

Chapter 3 presents the results of a study on the association between the physical environment characteristics of primary schools and the use of AST. Two out of three children used AST, and older children were more likely to use AST than younger children. The physical environment characteristics of primary schools were observed using a validated checklist and were clustered into four theory-based environmental categories: destination, safety, aesthetics, and functionality. For the young children (aged 5-9 years; grades 1-5) none of the environmental clusters were associated with AST. For the older children (aged 9-12 years; grades 6-8) however, the aesthetics cluster was positively associated with the use of AST. A safe school environment was considered to be conditional to this association, i.e. if a school environment is sufficiently safe, local aesthetics become important in predicting AST. Well-maintained local green spaces and the absence of litter were the most strongly associated characteristics within the aesthetics cluster. Local authorities can therefore support AST by paying sufficient attention to these aesthetic characteristics of the school environment.

The study discussed in chapter 4 also focused on the association between physical school environment characteristics and the use of AST among children aged 8-12 years. This study paid special attention to parental active transportation routines (PATRns). PATRns were both a positive associate of AST among children and a moderator of the association between environmental associates and AST. Distance was the strongest predictor of AST, greater distance being associated with fewer days on which a child travelled to school using AST. This association was moderated by PATRns. The barrier formed by distance was lower for children raised in families with strong PATRns, i.e. families where parents routinely walk and cycle. Conversely, the distance was more of a barrier for children raised in low-PATRns families. These findings imply that AST can be positively influenced by stimulating walking and cycling by parents in other contexts than school.

Chapter 5 describes a study in which children's objectively assessed PA was associated with the playability of the neighborhood in an 800 meter radius around primary schools. This study found that the playability of the school environment was 
associated with children's light and moderate-to-vigorous after-school PA. Playability explained approximately 30\% of the children's after-school PA. In addition, the strongest positive association between playability and children's PA levels was found for children living within 800 meters from their primary school. Time-specific analyses revealed that this association was particularly strong from the end of the school day up to $6 \mathrm{pm}$. These results indicate that a playable school environment contributes to children's after-school PA, and provide suggestions for health promotors and PA practitioners about when and where to plan their PA activities.

The study described in chapter 6 examined the association between the schoolyards' physical, social, and policy characteristics on the one hand and SB and moderate-to-vigorous PA at the schoolyard before, during and after school hours on the other. An innovative approach was used to merge objectively assessed PA data (accelerometry) and GPS data. This data merge enabled us to study children's PA and SB at the schoolyard in relation to its physical, social, and policy characteristics. It was found that children spent on average 54 minutes per school day at the schoolyard, 9 minutes (17\%) of which were spent in moderate-to-vigorous PA and 20 minutes (37\%) in SB. Boys were more active at schoolyards than girls. Moreover, the availability of fixed equipment, such as soccer goals and high bars, teachers' involvement in the initiation of PA activities and the presence of a ball game area policy were positively associated with more time spent in moderate-to-vigorous PA and less time spent in SB. By critically reconsidering the physical and social schoolyard environment and the policies that apply at the schoolyard, schools can contribute to promoting PA and reducing SB at the schoolyard.

Chapter 7 presents the results of a longitudinal study on the effectiveness of physical and social schoolyard interventions on the time spent in SB, light PA and moderate-tovigorous PA by primary school children aged 8-11 years at schoolyards during recess after 12 months. Children's PA levels were objectively assessed and a subsample of the children was additionally equipped with a GPS device. GPS confirmatory analyses showed that in 3 of the 10 school pairs at least one of the two (the intervention or control school) did not enable the children to visit the schoolyard during morning recess. These school pairs were therefore excluded from the study, resulting in the inclusion of 376 children in this study. A small positive effect was found on children's time spent in light PA in the intervention schools after 12 months (+5.9\%), largely explained by a decrease in time spent in SB (-5.4\%). The strongest predictor of this change was a larger number of physical schoolyard interventions. The effectiveness of the number of physical schoolyard interventions was moderated by the intensity of the interventions and the school's commitment to the project: interventions with a higher intensity and more commitment led to more positive results. No effects were found on time spent in moderate-to-vigorous PA. Based on these results, it would be useful to implement multiple physical schoolyard interventions to achieve sustainable changes in 
PA and SB, which requires ongoing attention being devoted to PA promotion and SB reduction at schoolyards.

Chapter 8 describes the effectiveness of the Active Living approach in terms of SB, light PA and moderate-to-vigorous PA after 12 months among children aged 8-12 years. The results were based on analyses of objectively assessed PA data of 520 children at 18 schools (nine intervention and nine control schools). Based on a robust exposure measure, i.e. exposed or not exposed to Active Living, no effects were found on the proportion of time spent in SB and/or PA. Active Living, however, was a tailored intervention approach leading to a wide variety of intervention packages among the intervention schools. Therefore, we adopted an analytic approach in which the variety of intervention packages was taken into account. These analyses were conducted with the number of physical and social environment interventions as independent variables, and we also tested the interaction between the physical and social environment interventions, to study potential synergetic effects. We found that children attending schools that implemented both sufficient physical environment interventions $(N \geq 4)$ and social environment interventions $(N \geq 6)$ spent 15 minutes less in SB during school days after 12 months. Moreover, these children spent 15 minutes more in light PA with a medium effect size (ES=0.41). We conclude that the Active Living approach has the potential to activate children, but a sufficient number of combined physical and social environment interventions is required to achieve effects after 12 months. When an insufficient number of changes were implemented in the physical and social environment, the program was not effective in increasing PA or decreasing SB. Based on these findings the main conclusion was reformulated as 'do it well or don't do it at all'.

Finally, chapter 9 of this thesis discusses the most important findings from the studies presented, and puts them in a broader perspective. In addition, this chapter presents the results of an additional follow-up study, which was conducted one year after the Active Living project in schools had ended (2015). The chapter also presents recommendations for future research and for practitioners involved in encouraging PA and reducing SB, based on the findings of the studies presented. The most important conclusion drawn from the studies in the thesis is that sustainable changes in PA and SB, i.e. changes lasting up to two years after implementation, can be achieved by physical and social environment interventions in primary schools, but only if these interventions meet specific conditions. Implementing multiple environmental changes leading to multiple PA stimuli seems an important condition for a sustainable change in children's PA and SB during school times.

A combined bottom-up and top-down approach seems an appropriate method to create local support for sustainable adjustments in the physical and social school environment. In addition, a synergetic effect can be achieved if sufficient physical and social environment interventions are implemented simultaneously. In other words, it would not only be useful to facilitate more PA-friendly physical school environments, but the use of the facilities available in the built environment should be stimulated 
(social environment) to ensure the greatest effectiveness of both types of interventions. This supports the conclusion formulated above for programs applying the method and changes used in Active Living, i.e. 'do it well or don't do it at all'. 



\section{Valorization Addendum}



The current chapter provides an overview of the societal value of the work presented in this thesis. This societal value will be described in terms of practical relevance of the study results, the target populations for whom these results are of interest, activities, products and innovations that are derived from the Active Living project. Finally, the dissemination of the results of the research and products will be described.

\section{RELEVANCE}

To date, over 70 percent of the Dutch children does not fulfill the Dutch guidelines for sufficient physical activity (PA) (Nederlandse Norm Gezond Bewegen; NNGB (Burghard, Knitel, van Oost, Tremblay, \& Takken, 2016)). Moreover, children show high levels of sedentary behavior (SB) in their daily patterns, both in school and during leisure time. In this thesis it was shown that primary school children spend over 16 hours per day in SB. Low levels of PA and high levels of SB are global threats to health and contribute to multiple diseases, such as overweight, cardiovascular diseases and mental well-being (Andersen et al., 2006; Ekelund et al., 2012; Kohl et al., 2012). Moreover, activity patterns developed during childhood are likely to track into adulthood (Parsons et al., 1999; Telama, 2009), indicating the urge to develop healthy PA-patterns during childhood. In addition, children raised in low socio-economic status environments tend to be at higher risks to be physically inactive (Gordon-Larsen et al., 2006). As children spend a substantial amount of time during the day at school, the school environment can contribute to the formation of these healthy physical activity patterns. A PAsupporting school environment can stimulate PA as well as reduce time spent in SB. Examples of a PA-supporting school environment are well-designed schoolyards, healthy school policies and a neighborhood design that facilitates active school transportation. In order to stimulate PA and reduce time spent in SB in primary schools located in low SES neighborhoods in the Southern-Limburg region of the Netherlands, the Active Living project was initiated and launched after the summer of 2012. The Active Living project focused on increasing PA and reducing SB in school, during leisure time and during active school transportation by means of changing the physical and social school environment. The studies presented in this thesis provide clear indications and suggestions how the school environment could be better designed to facilitate sustainable improvements in PA and reductions in time spent in SB. Several studies in this thesis indicated the need for tailored environmental interventions that fit to the needs of inhabitants of the neighborhoods rather than a one-size-fits-all approach. Moreover, indications were found for synergetic effects between the physical and social environment. Therefore, it might be concluded that designing the school environment as a whole rather than in a fragmented fashion is likely to be more effective in stimulating children's PA and reducing SB. 


\section{TARGET GROUPS}

The results presented in the thesis are of interest to multiple target populations. The results are informative to ZonMw (the Dutch Organization for Health Research and Development) and policy makers both on a national and local level. Clear indications were found that an integrative approach in which systems thinking was required led to promising results for PA promotion and SB reduction. Moreover, it was found that enduser's involvement in the development of interventions was important for the design, the implementation, and the sustainability of interventions.

Further, several studies presented indicated some suggestions for urban design, health and other municipal domains to support walking and cycling to school by means of creating or adapting the physical school environment. These suggestions can also be integrated in the work of the Public Health Services and other health promotion organizations. Based on the findings in this work it would be recommendable to consider the school environment as a whole system rather looking at the environment as a collection of single, fragmented pieces. Taking into account the broader perspective, the needs and the opportunities of school environments may lead to more synergetic effects of environmental interventions and sustainable effects on increasing PA and reducing SB. Besides, health promotion organizations may benefit from the finding that increasing active school transportation could be reached by targeting walking and cycling behavior by adults rather than focusing directly on children.

Schools can benefit from the results of the Active Living project by learning what physical and social environmental interventions might work to increase PA and reduce SB. Examples of such interventions are providing challenging play areas at schoolyards (e.g. soccer goals), implementing supportive policies in schools (e.g. extended opening hours of the schoolyard), and teacher-involvement in PA-activities. Results also indicate schools' predominant position in reducing physical inactivity as children spend large periods of time in SB during a school day. Focusing on schools' advantages of active and healthy children may be favorable to support schools to take action. However, the main finding in schools for increasing PA and reducing SB was to 'do it well or don't do it at all'. If schools are willing to take action in reducing PA it is strongly recommended to show high levels of commitment to the topic of PA and SB, in which several activities will be implemented to support children's PA and reducing their SB. A non-committed attitude of school personnel in facing the problem of physical inactivity is not likely to result in behavioral changes and might be even lead to decreased levels of PA over time.

The results from studies may also be relevant for parents. In the future, parents can be encouraged more often to choose a primary school for the children based on activity friendliness of the school environment, which supports the school to consider PAencouragement as a spearhead in the school policy to attract new pupils. Moreover, parents can learn by the finding that their own walking and cycling behavior is related to their child's use of active school transportation and as a result, their child's daily PA- 
levels. By giving the example of using active transportation or by actively accompanying children during their travel, parents may substantially contribute to children's PA.

\section{ACTIVITIES, PRODUCTS AND INNOVATIONS}

The Active Living project resulted in a couple of new activities and products, more easily referred to as the Active Living approach. The Active Living approach was characterized by the combination of a top-down and bottom-up approach, also known as mutual adaptation. This approach consisted of the formation of a working group at each participating school to optimize the content and form of the PA-interventions to local needs. Moreover, the approach was chosen to enhance sustainability of interventions by increasing the feeling of autonomy and ownership of the interventions in the working groups. This approach led to a tailored intervention package that shared the scope i.e. increasing PA and reducing SB, but differed in number, content and form across schools. The collaboration between researchers, health promotion organizations, local municipalities, school representatives and parents was supported by this approach and led to a coproduction for the intervention development and implementation. However, this coproduction is a non-traditional approach for experimental studies and could be considered as fairly innovative.

It should be noted that the composition of the working groups and their commitment to the project seem key factors in whether these groups succeed in implementing interventions. These working groups succeeded in implementing numerous PA-interventions in the school environment with a limited budget $(2,000$ euro at the start of the project). Herewith, these working groups have achieved to establish more playable, PA-supporting school environments. To date, changes in the physical environment do still exist in these school environments. The changes in the physical and social environment were not extremely large by extent and their level of innovativeness was limited, but the changes were easy to implement, fitted to local needs and were low-budgeted, which increases the likelihood of transferability of the interventions to other school environments with similar needs.

In addition, we used an innovative methodological approach that combined objectively assessed location-data (GPS) and PA-data (accelerometer). In collaboration with a world-leading institute in applying this technique (Southern Denmark University, Odense, Denmark), a real-life animation was developed in which time, children's location and activity level information were combined and presented on a map (Geographical Information System, GIS). By creating such an animation, it became visible where, when, and in what PA-intensity children performed PA. This real-life animation is innovative and very informative for health practitioners and policy makers to study human's PAbehavior on a daily basis in relation to the exposed environments. In practice, this tool has shown its usability in explaining and disseminating ideas to policy makers. 


\section{DISSEMINATION OF RESULTS AND PRODUCTS}

The outcomes of the work presented in this thesis have been disseminated through multiple channels. Firstly, the practical implications and working methods, including the combined bottom-up and top-down approach, has been presented to health promoters and municipal officers by means of multiple oral sessions throughout the Netherlands (e.g. oral presentation during 'Dag van het Sport Onderzoek (DSO), 2016', keynote lecture at the 'Provinciale Inspiratie Sessies ter bevordering van bewegingsonderwijs, 2016'). Moreover, the outcomes were disseminated by factsheets presented by the Regional Public Health Services Zuid Limburg, by a national journal publication and numerous media channels, including radio and TV.

Besides, the dissemination of the results of the Active Living project through media, readings and publications, the working methods, data analyses techniques and practical implications have been adapted in a couple of new (granted) projects. Examples of such projects are: KEIGAAF (Kansen in Eindhoven voor GezinsAAnpak met Fontys), granted by Fonds NutsOhra (FNO); SUPERFIT, granted by Fonds NutsOhra (FNO) and ZonMw; A2Health study, granted by Projectbureau A2 Maastricht together with the Faculty of Health, Medicine and Life Sciences, University Maastricht. Moreover, the Healthy Elementary School of the Future follow the basic assumptions of the Active Living project. From these project, the KEIGAAF project (a collaboration between Maastricht University and Fontys University of Applied Sport Sciences) is the most similar to Active Living. The school-based part of the KEIGAAF project is completely based on the Active Living project, including the Active Living approach to develop and implement interventions. In addition to the Active Living project, an evidence-based parental intervention component (Lifestyle Triple P) will be added to the KEIGAAF project.

Besides dissemination through projects, the outcomes from the Active Living project and the Active Living approach will be used for educational purposes. For instance, the approach will be integrated as part of a module in the new curriculum for the Academy of Physical Education at Fontys University of Applied Sport Sciences.

In addition, it should be noted that although the project assistance for Active Living schools was granted until summer 2014, to date many participating schools continue running their working group at school level. Some of the working groups broadened their focus to nutrition behavior in addition to PA. One school expanded their activities largely and is now one of the four participating schools in the Healthy Elementary School of the Future project. 


\section{Appendices}




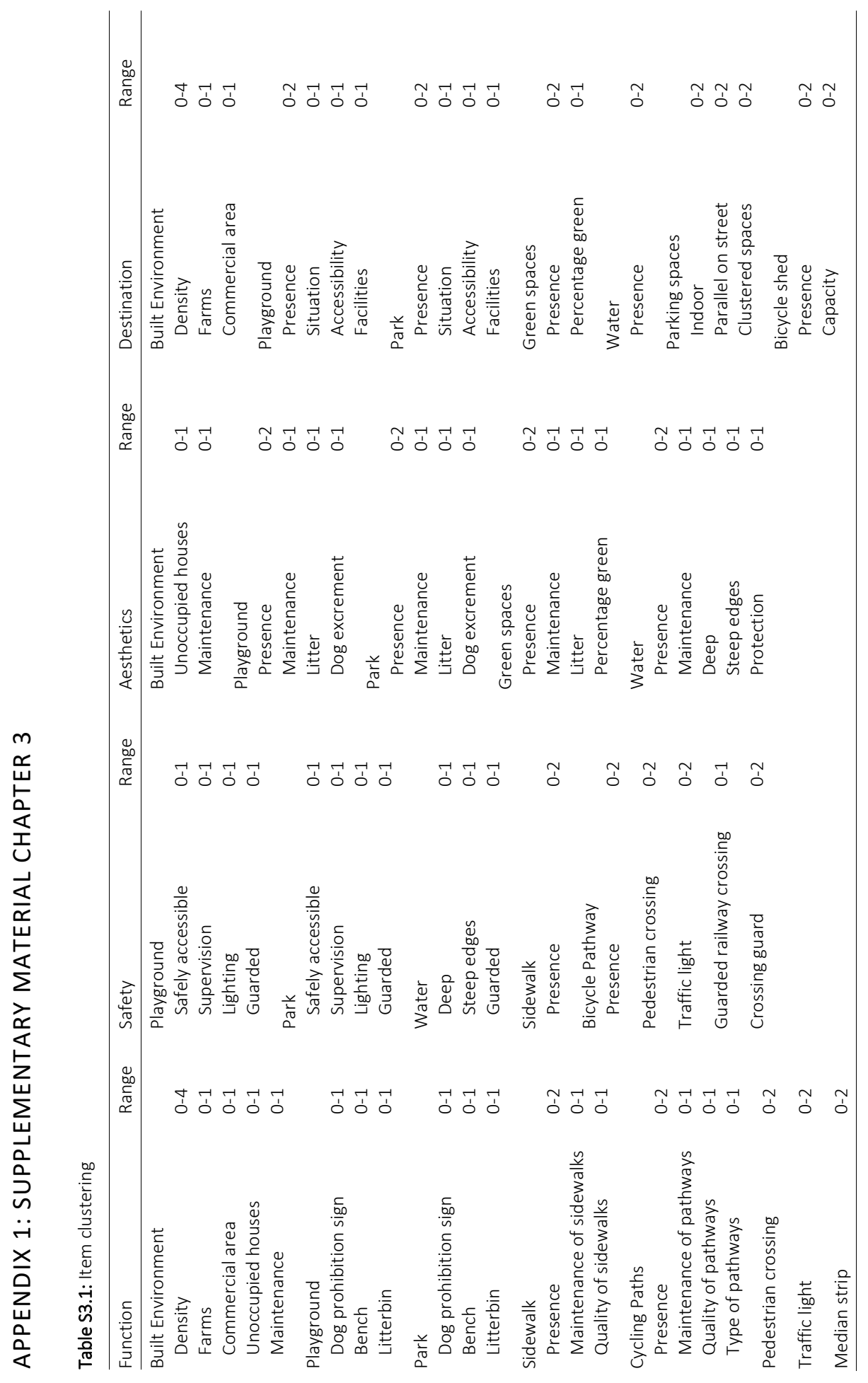




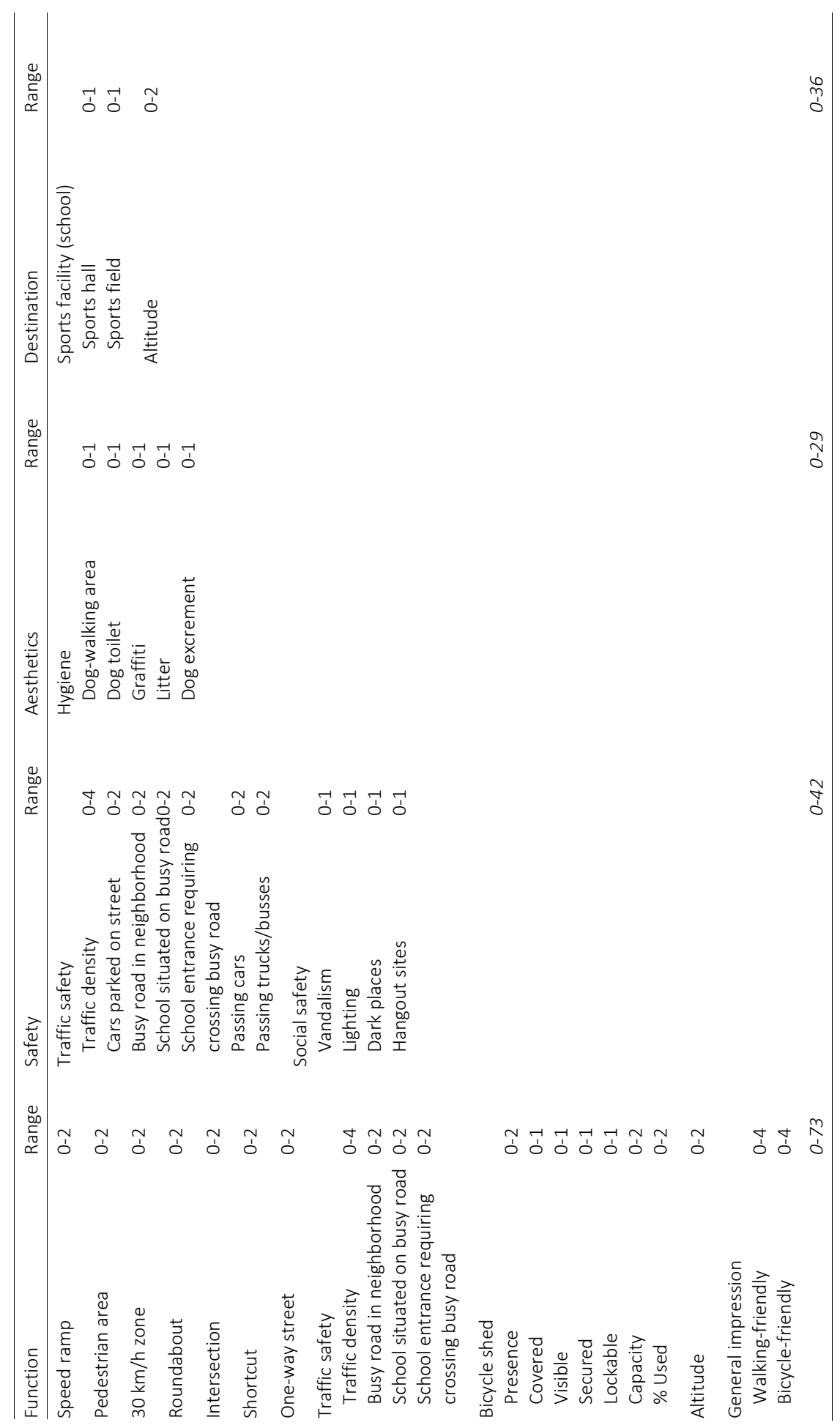




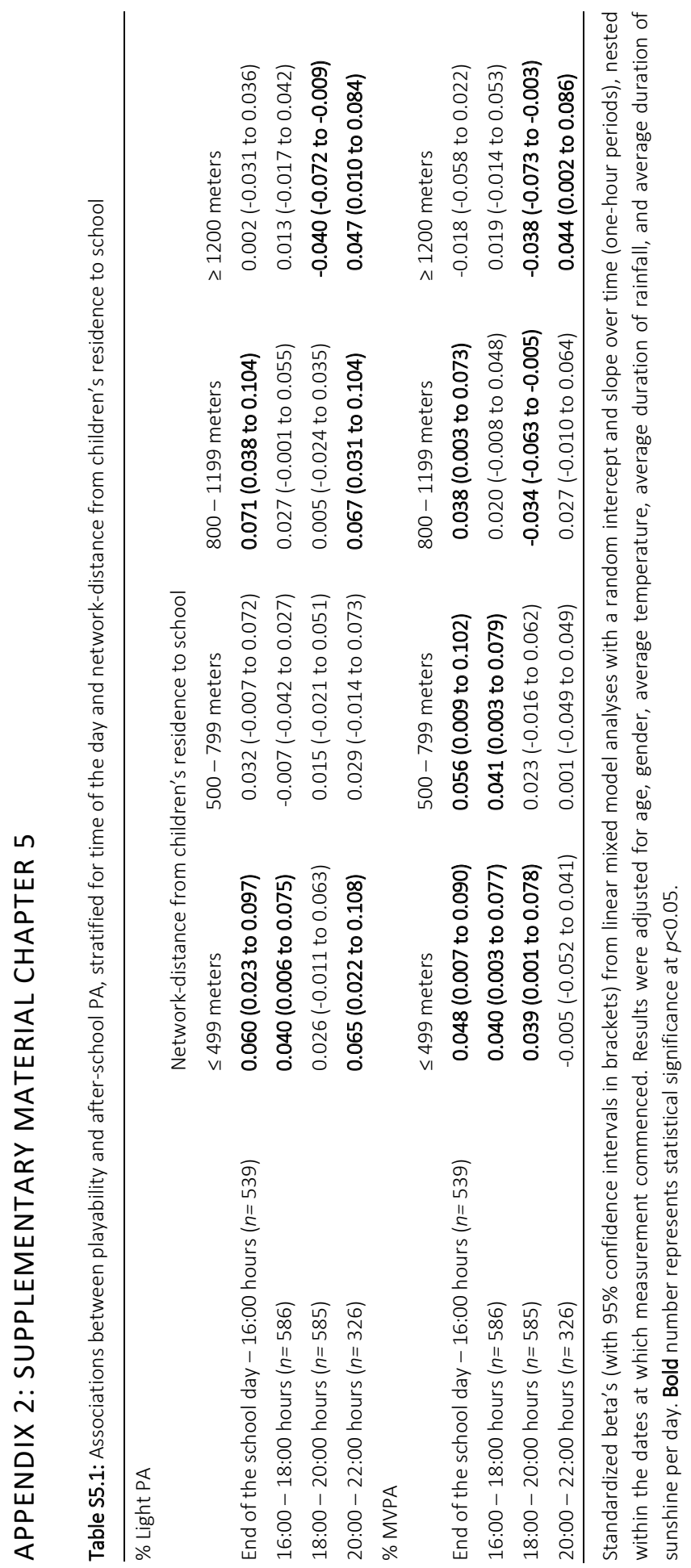




\section{APPENDIX 3: SUPPLEMENTARY MATERIAL CHAPTER 8}

Table S8.1: Overview of implemented interventions per domain per school

\begin{tabular}{l} 
Active transportation to school \\
\hline Physical Environment
\end{tabular}

\begin{tabular}{|c|c|c|c|c|c|c|c|c|c|}
\hline PA in school & & & & & & & & & \\
\hline Physical Environment & & & & & & & & & \\
\hline New fixed equipment in schoolyard & - & + & - & + & + & + & - & + & + \\
\hline New loose equipment in schoolyard & - & + & + & - & + & + & + & - & + \\
\hline Playground markings & - & + & - & - & - & + & + & - & - \\
\hline Establish ball game area & + & + & - & - & - & + & - & + & - \\
\hline Put a ball backstop besides railway & + & - & - & - & - & - & - & - & - \\
\hline Sound equipment in schoolyard environment & - & + & - & - & - & - & - & - & - \\
\hline Social Environment & & & & & & & & & \\
\hline Additional sports day in schoolyard & + & + & + & + & - & - & - & + & - \\
\hline Sports clinics in recess & + & + & + & + & + & + & - & - & + \\
\hline Use of schoolyard games & - & + & + & - & - & - & + & + & - \\
\hline Prize contest for best idea PA stimulation children & + & - & - & - & - & - & - & + & - \\
\hline PA in leisure time & & & & & & & & & \\
\hline Physical Environment & & & & & & & & & \\
\hline Establish training circuit & - & - & + & - & - & - & - & - & - \\
\hline Social Environment & & & & & & & & & \\
\hline Active Living Games & + & + & + & + & + & + & + & - & + \\
\hline Establish out-of-school PA program & + & + & + & + & + & - & - & + & + \\
\hline Establish school soccer team & - & - & + & - & - & - & - & - & - \\
\hline $\begin{array}{l}\text { Establish PA activities by children for local } \\
\text { residents }\end{array}$ & - & - & + & - & - & - & - & - & - \\
\hline Physical Environment Total & 4 & 8 & 6 & 3 & 3 & 6 & 2 & 2 & 4 \\
\hline Social Environment Total & 6 & 6 & 10 & 7 & 4 & 3 & 3 & 4 & 3 \\
\hline
\end{tabular}

Note: $I_{n}=$ Intervention school (number); + = implemented; - = not implemented 



\section{Dankwoord}



Het dankwoord is het enige hoofdstuk in een proefschrift waar een niet-nuttige uitweiding wordt toegestaan en gewaardeerd. Waarschijnlijk is het dankwoord ook een van de meest gelezen hoofdstukken van een gemiddeld proefschrift. Dit proefschrift zal daar naar alle waarschijnlijk geen uitzondering op vormen en daarom heb ik mijn uiterste best gedaan om iedereen die een substantiële bijdrage heeft geleverd aan de totstandkoming van dit boekwerk bij naam te noemen in dit hoofdstuk.

Ik heb het voorrecht gehad om een praktijkgericht onderzoek te mogen opzetten, uitvoeren en evalueren. Het Active Living project is een coproductie van vele organisaties en mensen, wat leidt tot een omvangrijk dankwoord aan allen die ik dank verschuldigd ben.

Om te beginnen mijn promotieteam, Maria, Nanne, Sanne en Stef. Maria, dank je wel voor je in mij gestelde vertrouwen vanaf het moment van de sollicitatie. Ook wil ik je bedanken voor alle vrijheid en ruimte die je me in het project hebt geboden. Of ik nu mijn computer wilde laten uitbreiden om met GPS data te 'spelen' of dat ik had geregeld dat ik op werkbezoek kon naar Denemarken, je hebt het altijd gefaciliteerd en gestimuleerd. Nanne, de stille motor op de achtergrond van het project. Nou ja, stil... Jouw scherpe kijk op stukken en enthousiasme over het project hebben mij flink geholpen. Ook wanneer er zaken stroef liepen, was jij meteen bereid om 'een telefoontje' te plegen waarmee je paden plaveide. Sanne, wat een voorrecht om één van de meest vooraanstaande onderzoekers in Nederland op dit thema in mijn promotieteam te mogen hebben. Naast je inhoudelijke expertise over (niet) bewegende kinderen op en rondom scholen, heb ik veel van je geleerd over hoe om te gaan met accelerometers. Jouw TNO netwerk was hierin ook van grote waarde. Ik kijk met veel plezier uit naar het voortzetten van onze samenwerking via de Haagse Hogeschool en Fontys Sporthogeschool. Jullie drieën samen maakten overigens dat ik op (buitenlandse) congressen en symposia nooit hoefde uit te leggen uit welk land ik kwam; Jansen, de Vries, \& de Vries, hartelijk dank!

Stef, bij het vermelden van jouw naam hoefde ik ook nooit uit te leggen waar ik vandaan kwam. Je naam werd altijd direct herkend als inhoudelijk expert op het gebied van obesitaspreventie. Aan de andere kant werd je naam ook herkend als 'isn't that the guy who was dancing in our symposium?', één van de vele befaamde acties tijdens congresbezoeken. Jouw humor, inhoudelijke kijk op de materie en het relativeringsvermogen waarmee jij het werk uitvoert, zijn een ware inspiratiebron voor me geweest. Je hebt me meteen in het eerste jaar van mijn promotietraject 'geadopteerd' als CAPHRI-telg in de NUTRIM-familie. Je hebt me gestimuleerd en gefaciliteerd om de halve wereld over te reizen voor congressen en symposia en je schuwde er niet voor om me 'voor de leeuwen te gooien' als jij dacht dat ik iets zou kunnen. Jouw vertrouwen in mij heeft mij als persoon en mijn carrière enorm geholpen. Daarnaast was je altijd bereid om met me te sparren wanneer ik weer een 'ideetje' had. Niet elk 'ideetje' was 
misschien even doordacht en kansrijk, maar het heeft tot leuke discussies geleid en in de vorm van KEIGAAF en de A2Health studie tot concrete vervolgprojecten. Ik hoop onze samenwerking nog lang te mogen continueren. Mijn dank is ontzettend groot dat je me gedurende het hele traject gesteund, geïnspireerd en uitgedaagd hebt.

Naast het promotieteam wil ik graag mijn dank uitspreken aan Jasper (Schipperijn). Wat begon als een toevallige ontmoeting in San Diego in 2013, leidde tot een mooie samenwerking tussen Odense en Maastricht. Ik ben blij dat ik tweemaal jouw vakgroep heb mogen bezoeken en heb mogen leren van jouw kennis en kunde met betrekking tot de verwerking van GPS data en accelerometrie. Veel dank voor de Deense gastvrijheid.

Daarnaast wil ik graag mijn dank uitspreken aan de leden van de beoordelingscommissie van dit proefschrift voor hun inzet tijdens het beoordelingsproces. Dike, Martin, Gerjo, Esther en Patricia, hartelijk dank!

Een praktijkgericht onderzoek is niets zonder een belangrijke praktijkpartner, in dit geval de GGD Zuid Limburg. Nicole en Petra, als projectleiders van Active Living hebben jullie vele deuren geopend die vaak voor een onderzoeker gesloten blijven, dank daarvoor en uiteraard ook voor de fijne samenwerking. Verder gaat mijn dank nog uit naar Patricia, Judith, Nickee, Truusje, Githe, Rick, Fleur, Marlou, Mandy, Nina die in verschillende vormen een mooie bijdrage hebben geleverd vanuit de GGD. Daarnaast spreek ik ook mijn dank en waardering uit voor Ingrid, Conny en Kyra. Jullie secretariële ondersteuning was van grote waarde.

Over praktijk gesproken; de bijdrages, inzet en betrokkenheid van alle deelnemende Zuid-Limburgse gemeentes en deelnemende scholen zijn van enorm belang geweest voor het Active Living project. Verder spreek ik nog veel dank uit aan alle deelnemende kinderen en hun ouders! Zonder jullie zouden we als onderzoekers helemaal niets kunnen bestuderen. Het invullen van meerdere vragenlijsten en het meermaals dragen van de meetapparatuur wordt enorm gewaardeerd.

Susanne, ik weet niet hoeveel kilometers jij door het Zuid-Limburgse land hebt gereden voor het bijwonen van zowat alle metingen door de jaren heen en het ophalen van alle meters. Heel veel dank voor alle uurtjes die jij in het project hebt gestopt en bovendien vooral bedankt voor de leuke samenwerking.

Patricia, Kim, Marja, Leon, de (voormalig) ondersteuntoppers van de vakgroep gezondheidsbevordering. Altijd stond jullie deur voor mij open wanneer ik een hulpvraag had. Naast hulp hebben jullie vooral ook veel bijgedragen aan de humorvolle momenten door de GB-jaren heen. Dank je wel voor alles!

Teun, Rik, Eva, mijn roomies door de GB-jaren heen. Dank voor jullie gezelligheid op de kamer, waarbij de prijs voor dubieuze inrichtingskwaliteiten van de kamer en het oog 
daarbij voor spuuglelijke details toch absoluut naar Eva gaat. Verder wil ik alle (ex-)collega's van de vakgroep gezondheidsbevordering bedanken voor alle leuke momenten die we samen hebben mogen meemaken de afgelopen jaren. Sacha en Nicole, ik vind het ontzettend leuk dat ik jullie mag begeleiden en ik leer nog elke dag van jullie. Dank jullie wel voor jullie enthousiasme in de projecten!

Steven, Dianne, Lars, Gertjan en Matty, jullie hebben een belangrijke rol gespeeld in de keuze om mij aan te stellen in Eindhoven. Ik ben jullie hier dankbaar voor en geniet nog elke dag van de vrijheid die jullie mij geven om mijn werk bij Fontys Sporthogeschool uit te voeren. Ik hoop nog een hele tijd met jullie samen te mogen werken, want het is KEIGAAF.

Rik en Vincent, heel veel dank voor alle gezellige momenten die ik met jullie heb mogen meemaken, zowel binnen de muren van DEB1 als ook daarbuiten. Ik ben heel blij dat jullie mij ondersteunen als paranimfen!

Lieve mama, Ferry en Helene, ontzettend bedankt voor jullie eeuwige steun in goede en slechte tijden! Jullie zijn voor mij het levende bewijs dat qua familiaire steun kwaliteit absoluut boven kwantiteit gaat. Verder spreek ik nog graag mijn dank uit aan Dorien, Ruud en Marieke voor jullie interesse gedurende het hele promotietraject.

Rest mij tot slot nog één persoon te bedanken, Sanne! Mijn dank aan jou voor al je steun en liefde is ontzettend groot en eigenlijk helemaal niet in woorden te vatten. Vanaf dag 1 (of eigenlijk daarvoor al) heb je me altijd geïnspireerd om dit proces goed te doorlopen, hoewel jij als ervaringsdeskundige weet dat dit niet altijd even eenvoudig is. Van medestudent, naar collega, naar mijn vrouw; er is maar één persoon op de wereld die dat rijtje kan opnoemen en dat ben jij. Ik kijk met heel veel plezier vooruit naar alle mooie dingen die de toekomst ons hopelijk nog gaat brengen. Ik hou van je!

Dave 



\section{Curriculum Vitae}



Dave Helene Hub Van Kann was born on March $9^{\text {th }} 1985$ in Kerkrade, the Netherlands. In 2007 he graduated from the Bachelor Health Sciences at Maastricht University with a major in Health Education and Promotion and minor in Movement Sciences. One year later he graduated from the Master Public Health at Maastricht University with a specialization in Health Promotion.

He started working as research assistant at the department of Health Promotion at Maastricht University in May 2009. In October 2011, he started his PhD-project in collaboration with the Regional Public Health Services at the same department. During his PhD-trajectories he performed multiple teaching activities at Maastricht University, collaborated in several grant proposals and acted as peer-reviewer for several scientific international journals.

In January 2016, he started working as lecturer and researcher at Fontys University of Applied Sport Sciences in Eindhoven. Currently, he is project leader of the KEIGAAF project. Moreover, he is project leader of a longitudinal study in which the effectiveness of qualified physical education teachers on children's physical activity in Dutch primary schools will be studied. In addition, he coordinates the development of a new fulltime master physical education and co-supervises two PhD candidates at Maastricht University. 



\section{Publication List}




\section{PUBLICATIONS PRESENTED IN THIS THESIS}

Van Kann, D. H. H., de Vries, S. I., Schipperijn, J., de Vries, N. K., Jansen, M. W. J. \& Kremers, S. P. J. A multicomponent schoolyard intervention targeting children's recess physical activity and sedentary behavior: Effects after one year. Submitted for publication.

Van Kann, D. H. H., de Vries, S. I., Schipperijn, J., de Vries, N. K., Jansen, M. W. J. \& Kremers, S. P. J. (2016). Schoolyard characteristics, physical activity and sedentary behavior: Combining GPS and accelerometry. Journal of School Health, 86(12), 913-921.

Van Kann, D. H. H., Kremers, S. P. J., de Vries, N. K., de Vries, S. I. \& Jansen, M. W. J. (2016). The effect of a multicomponent intervention on physical activity and sedentary behavior in primary school children: the Active Living study. Preventive Medicine, 89, 64-69.

Remmers, T., Van Kann, D., Thijs, C., de Vries, S. \& Kremers, S. Playability of the neighborhood environment and after school physical activity among 8-11 year-olds. (2016). International Journal of Physical Activity and Behavioral Nutrition, 13:82.

Van Kann, D. H. H., Kremers, S. P. J., De Vries, S. I., De Vries, N. K. \& Jansen M. W. J. (2016). Parental Active Transportation Routines (PATRns) as a moderator of the association between neighborhood characteristics and parental influences and active school transportation. Environment and Behavior, 48(7), 946-965.

Van Kann, D. H. H., Jansen, M. W. J., de Vries, S. I., de Vries, N. K. \& Kremers, S. P. J. (2015). Active Living: Development and quasi-experimental evaluation of a schoolcentered physical activity intervention for primary school children. BMC Public Health, 13:1315.

Van Kann, D. H. H., Kremers, S. P. J., Gubbels, J. S., Bartelink, N. H. M., De Vries, S. I., De Vries, N. K. \& Jansen M. W. J. (2015). The association between the physical environment of elementary schools and active school transport. Environment and Behavior, 47(4), 418-435.

\section{OTHER PEER-REVIEWED INTERNATIONAL JOURNAL PUBLICATIONS}

Gerards, S. M. P. L., Van Kann, D. H. H., Jansen, M. W. J. \& Kremers, S. P. J. The interaction between parenting practices and the physical environment on changes in child physical activity and sedentary behavior - a longitudinal study. Submitted for publication.

Soini, A., Gubbels, J., Sääkslathi, A., Villberg, J., Kremers, S., Van Kann, D., Mehtälä, A., de Vries, N., \& Poskiparta, M. (2016). A comparison of physical activity levels in childcare 
context among Finnish and Dutch 3-years-olds. European Early Childhood Education Research, 24(5), 775-786.

Niermann, C. Y. N., Hermann, C., von Haaren, B., Van Kann, D. \& Woll, A. (2016). Affect and Subsequent Physical Activity: An Ambulatory Assessment Study Examining the Affect-Activity Association in a Real-Life Context. Frontiers in Psychology, 7:677.

Janssen, E., Van Kann, D., de Vries, H., Lechner, L. \& van Osch, L. (2015). Sun Protection during snow sports: An analysis of behavior and psychosocial determinants. Health Education Research, 30(3), 380-387.

Gubbels, J. S., Van Kann, D. H. H., De Vries, N. K., Thijs, C. T. \& Kremers, S. P. J. (2014). The next step in health behavior research: the need for ecological moderation analyses - an application to diet and physical activity at childcare. International Journal of Behavioral Nutrition and Physical Activity, 11:52.

Remmers, T., Van Kann, D., Gubbels, J., Schmidt, S., de Vries, S., Ettema, D., Kremers, S. \& Thijs, C. (2014). Moderators of the Longitudinal Relationship between the Perceived Physical Environment and Outside Play in Children: The KOALA Birth Cohort Study. International Journal of Behavioral Nutrition and Physical Activity, 11:150.

Gubbels, J. S., Van Kann, D. H. H. \& Jansen, M. W. J. (2012). Play equipment, physical activity opportunities, and children's activity levels at childcare. Journal of Environmental and Public Health. DOI: 10.1155/2012/326520

Gubbels, J. S., Kremers, S. P. J., Van Kann, D. H. H., Stafleu, A., Candel, M. J., Dagnelie, P. C., De Vries, N. K. \& Thijs, C. T. (2011). Interaction between physical environment, social environment and child characteristics in determining physical activity at child-care. Health Psychology, 30(1), 84-90.

\section{NATIONAL JOURNAL PUBLICATIONS}

Van Kann, D., Vos, S., \& Kremers, S. (2016). Waar bewegen basisschoolleerlingen eigenlijk? Nieuwe technieken leveren nieuwe inzichten op. Lichamelijke Opvoeding, 8, 19-21.

Walthouwers, M., Olberding, M., Hoving, C., Op de Coul, M., Janssen, E., Van Kann, D., Lechner, L., de Vries, H. \& van Osch, L. (2012). Kennis van kankersymptomen en de rol van psychosociale factoren bij tijdig medisch hulpzoekgedrag. TSG, 90, 36-44.

Gubbels, J. S., Kremers, S. P. J., Thijs, C. T., Stafleu, A., Van Kann, D. H. H., de Vries, S. \& de Vries, N. K. (2010). Overgewicht bij jonge kinderen: De invloed van het kinderdagverblijf op voeding en beweging. Jeugd en Co: Kennis, 4, 15-23. 


\section{BOOK CONTRIBUTION AND SCIENTIFIC REPORTS}

Willemsen, M., Van Kann, D. \& Janssen, E. Stoppen met roken: Ontwikkeling, implementatie en evaluatie van een massamediale campagne. In: Brug, J., van Assema, P. \& Lechner, L. (2012). Gezondheidsvoorlichting en gedragsverandering: Een planmatige aanpak.

Van Kann, D., Janssen, E., van Osch, L. \& de Vries, H. (2011). Evaluatie van de publiekscampagne 'Vroege Ontdekking van Kanker': 'Ken de 9 signalen!'. Maastricht; Caphri, Maastricht University.

Van Kann, D., Janssen, E., van Osch, L. \& de Vries, H. (2011). Usability-test Kanker Risico Test: 'Van ontwikkeling naar probleemidentificatie'. Maastricht; Caphri, Maastricht University.

Van Kann, D., Janssen, E., van Osch, L., Lechner, L. \& de Vries, H. (2011). Evaluatie van de publiekscampagne 'Verstandig Zonnen 2010': 'Geniet van de zon, maar zorg dat je niet verbrandt.' Maastricht; Caphri, Maastricht University.

Van Kann, D. \& de Vries, H. (2010). Evaluation report ACCESS-project. Maastricht; Caphri, Maastricht University.

Van Kann, D., Janssen, E., van Osch, L. \& de Vries, H. (2010). Evaluatie van de publiekscampagne '6x sterker tegen kanker 2010': 'Wat doe jij om je risico op kanker te verkleinen?'. Maastricht; Caphri, Maastricht University.

Van Kann, D., Janssen, E., van Osch, L., Lechner, L. \& de Vries, H. (2010). Evaluatie van de publiekscampagne '6x sterker tegen kanker 2009-2010': 'Hoeveel keer sterker ben jij tegen kanker?'. Maastricht; Caphri, Maastricht University.

Van Kann, D., Janssen, E., van Osch, L., Willemsen, M. \& de Vries, H. (2010). Evaluatie SMR-campagne 2009-2010: 'In iedere roker zit een stopper.'. Maastricht; Caphri, Maastricht University.

Van Kann, D., Janssen, E., van Osch, L., Lechner, L. \& de Vries, H. (2009). Evaluatie Campagne Vroege Ontdekking 2009: 'Ken de 9 signalen!'. Maastricht; Caphri, Maastricht University.

Van Kann, D., Janssen, E., van Osch, L., Lechner, L. \& de Vries, H. (2009). Zonverstandig: Evaluatie van de KWF zoncampagne 2009. Maastricht; Caphri, Maastricht University. 


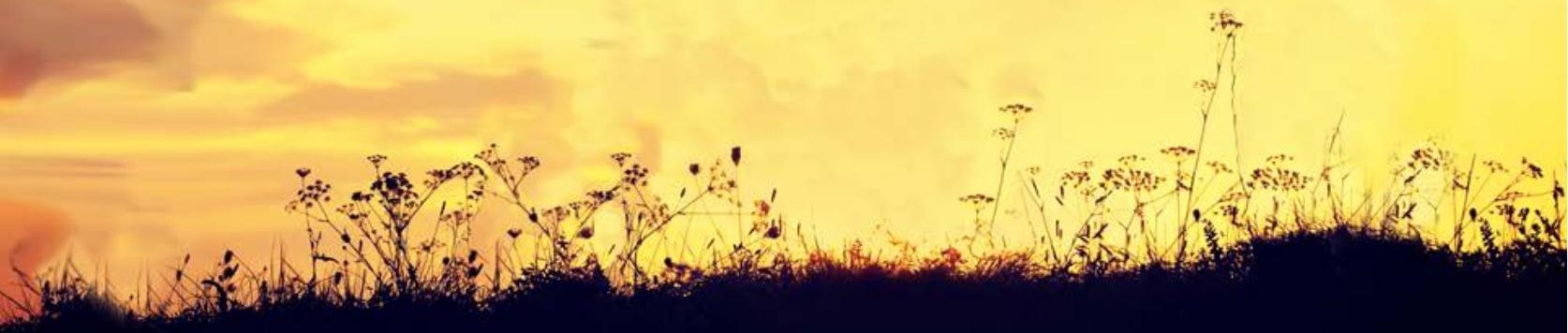

